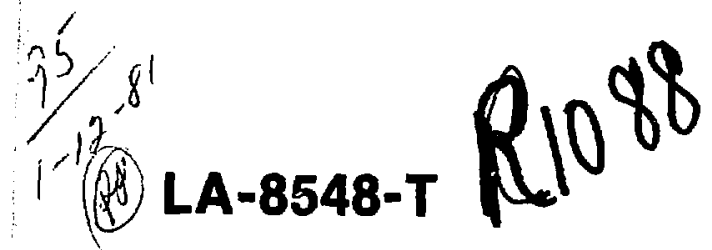

Dr. 2186

Thesis

Relativistic Off-Mass-Shell and Off-Energy-Shell Scattering Theories: An Application of the Rotation and Lorentz Groups 
An Affumative Action/Equal Opportunity Employer

This thesis was accepted by The University of Texas at Austin in partial fulfillment of the requirements for the degree of Doctor of Philosophy in Physics. It is the independent work of the author and has not been edited by the Technical Information staff.

This work was supported by the Associated Western Universities. ment. Neither the United States Government not any agency thereof, not any of their employees, makes any warranty, express or implicd, or assumes any legal liability or responsibility for the aceuracy, completeness, or usefulness of any information, apparatus, product, or process disclosed, or represents that its use would not infringe privately owned rights. Reference herein to any specific commercial product, process, or service by Irade name, trademark, manufacturer, or otherwise, does not necesearily constitute or imply its endorsement, recommendation, of favoring by the United States Government or any agency thereof. The views and opinions of authors expressed herein do not necessarily state or refect those of the United States Government or any agency thereof. 
LA-8548-T

Thesis

UC-34d

Issued: October 1980

\section{Relativistic Off-Mass-Shell and Off-Energy-Shell Scattering Theories: An Application of the Rotation and Lorentz Groups}

D. R. Giebink*

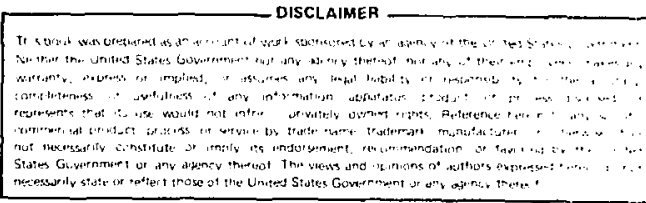

* Guest Scientist. Nuclear Theory Group, Department of Physics, University of Maryland, College Park, MD 20742.

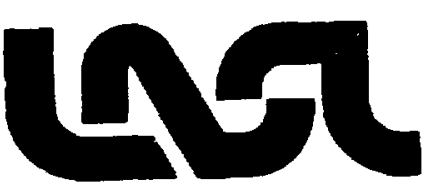


CONTENTS

ABSTRACT

INTRODUCTION

Chapter 1 THE ROTATION AND LORENTZ GROUPS

1.1 Rotations: SO(3) 17

$\begin{array}{lll}1.2 & \text { Rotations: SU(2) } & 18\end{array}$

1.3 Parameterizations of the Rotation Groups 21

$\begin{array}{lll}1.3 .1(\phi, \hat{\mathrm{n}}) \text { Parameterization } & 21\end{array}$

1.3.2 Euler-Rodriguez Parameterization 22

1.3.3 Euler-Angle Parameterization 22

1.4 Higher-Dimensional Representations of SU(2) 25

1.4.1 Orthogonality of $\mathrm{D}^{S}(R) \quad 27$

1.5 Lorentz Transformations: $\operatorname{SO}(3,1) \quad 29$

1.6 Lorentz Transformations: $\operatorname{SL}(2, C) \quad 32$

1.7 Higher-Dimensional Representations of SL(2,C) 34 
Chapter 2 RELATIVISTIC SINGLE-PARTICLE STATES WITH SPIN

2.1 The Definition of Single-Particle State Vectors

2.1.1 Z Basis

2.1.2 Helicity Basis

2.2 Lorentz Transformations of Single-Paricle States

2.2.1 Lorentz Transformations: $Z$ Basis 46

2.2.2 Lorentz Transformations: Helicity Basis 49

2.3 Improper Transformations $\quad 52$

2.3.1 Space Inversion (Parity) 53

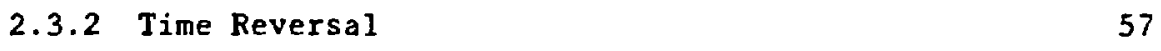

Chapter 3 RELATIVISTIC TWO-PARTICLE STATES WJTH SPIN 60

3.1 The Kinematics of Two-Particle States 61

3.2 The Definition of Two-Particle State Vectors 66

3.3 The Spin of Two-Particle States:

Angular-Momentum Expansions $\quad 70$

3.3.1 Z Basis 71

3.3.2 Helicity Basis 73

$\begin{array}{lll}3.3 .3 & \text { Permutations } & 75\end{array}$

3.4 Improper Transformations of Two-Particle States 76 
$\begin{array}{lll}\text { Chapter } 4 & \text { SCATTERING THEORY } & 79\end{array}$

4.1 The S Matrix and the R Matrix 82

$\begin{array}{lll}4.2 & \text { The M Functions } & 86\end{array}$

4.2.1 Single-Particle Crossing 89

4.3 View from a Time-Ordered World 91

4.3.1 A Note on Stapp's M Function 99

$\begin{array}{ll}4.4 & \text { Invariance Principles } \\ & 100\end{array}$

4.4.1 S-Matrix Invariance Principles 100

4.4.2 M-Function Invariance Principles 102

4.5 An Example: The rNN Vertex 104

$\begin{array}{ll}\text { 4.5.1 Crossing Properties } & 107\end{array}$

$\begin{array}{ll}\text { 4.5.2 Invariance Properties } & 109\end{array}$

Chapter 5 THE TWO-BODY PROBLEM:

ON-SHEIL, OFF-MASS-SHELL, AND OFF-ENERGY-SHEII 110

5.1 The R Matrix: On-Shell 114

5.1.1 Total-Angular-Momentum Representations 113

$\begin{array}{ll}5.1 .2 & \text { Space-Inversion Invariance } \\ \end{array}$

5.1.3 Time-Reversal Invariance 117

5.2 The Center-of-Mass M Function: Off-Mass-Shell 117

5.2.1 Total-Angular-Momentum Representations 121

5.2.2 Space-Inversion Invariance 124

5.2.3 Time-Reversal Invariance 126 
5.3 The T Function: Off-Mass-She?l 127

5.4 The Two-Body Bethe-Salpeter Equation 130

5.5 Reduction of the Two-Body Bethe-Salpeter Eguation

to Lippmann-Schwinger Form 132

5.5.1 Unitarity 133

5.5.2 The Off-Energy-Shel.1 Vertex Functions 137

$\begin{array}{ll}5.6 \text { Covariant Phenomenology } & 140\end{array}$

5.6.1 Covariant Off-Energy-Shell Continuation: Spin-0 141

5.6.2 A Dispersion-Theorectic Off-Energy Shell

$\begin{array}{ll}\text { T Function: Spin } 0 & 150\end{array}$

5.6.3 Phenomenolgy with Spin 153

Chapter 6 INTRODUCTION TO THE RELATIVISTIC THREE-BODY PROBIEM 162

6.1 The Kinematics of Three-Particle States 164

6.2 The Definition of Three-Particle State Vectors 169

6.3 The M Function Recoupling Coefficient 173

6.3.1 More Three-Body Kinematics 180

6.3.2 Return to the M Function Recoupling Coefficient 187

6.4 The T Function Recoupling Coefficient 189

6.5 Isospin 193 
Appendix A MISCELLANEOUS RESULTS CONCERNING ROTATION MATRICES AND CLEBSCH-GORDAN COEFFICIENTS

Appendix B SO(3) $\leftrightarrow$ SU(2)

199

Appendix C DETAILS FROM CHAPTER 3

C-1 Proof of Eq. 3-5

C-2 Proof of Eq. 3-7

205

C-3 Proof of Eqs. $3-34$ and 3-37

206

C-4 Proof of Eq. 3-36

208

C-5 Proof of Eq. 3-38

209

C-6 Proof of Eq. 3-41

Appendix D FIELD THEORY

212

D-1 The Fields

212

D-2 Causality

217

D-3 Antiparticles 220

D-4 Space Inversion, Time Reversal, and Charge Conjugation

D-5 2(2s+1) Component Fields

223

D-6 The Propogator

224

D-7 The M Function

227 
Appendix E DETAILS FROM CHAPTER $6 \quad 230$

$\begin{array}{lll}\text { E-1 Proof of Eq. 6-69 } & 230\end{array}$

E-2 Proof of Eq. 6-22 232

$\begin{array}{ll}\text { ACKNOWLEDGMENTS } & 235\end{array}$

$\begin{array}{ll}\text { REFERENCES } & 236\end{array}$ 
RELATIVISTIC OFF-MASS-SHELI AND OFF-ENERGY-SHELL

SCATTERING THEORIES: AN APPLICATION OF THE

ROTATION AND LORENTZ GROUPS

by

D. R. Giebink

ABSTRACT

A relativistic, phenomenological scattering theory for particles with arbitrary spin is presented, and the relation between off-mass-shell and off-energy-shell theories is discussed. The theory is formulated from the Hilbert-space representation of particles with spin in relativistic quantum mechanics. This topic is reviewed in a basis independent manner by appealing to the properties of the rotation and Lorentz groups and their representations. Spin is discussed and a set of basis state vectors for the singleparticle Hilbert space is derived from this perspective. Two- and three-particle Hilbert-space bases are then constructed, and angular nomentum is discussed in complete analogy with the single-particle 
states. The $\hat{\mathbf{z}}$ and helicity bases are presented as examples of the general procedure. These foundations permit a thecretically meaningful discussion of relativistic scattering theory and allow the on-shell scattering amplitude to be defined. The space-inversion and time-reversal properties of this amplitude suggest that a rew scattering function be defined such that a continuation of that function to negative energies can be considered. Antiparticle scattering events are associated with the continued function, and the CPT theorem arises as a natural consequence of this association. Moreover, these considerations lead to the definition of an offmass-shell scattering function. The resulting off-mass-shell scattering theory, which is shown to be essentially equivalent to perturbative field theory, has a number of very appealing properties. First, only $2 \mathrm{~s}+1$ spin-projection indices are required to describe a particle with spin $s$ in the off-mass-shell scattering function, and particles with any spin are described by exactly the same formalism. Second, it is an off-mass-shell theory, and the Lorentz transformation properties of the off-mass-shell scattering function are well defined. Third, the theory is easily subjected to reduction techniques that allow for the development of an equivalent off-energy-shell scattering theory. The off-energy-shell theory is dependent on fewer variables than the off-mass-shell theory and is more susceptible to a phenomenological treatment. 
Thus, phenomenology is discussed and dispersion-theoretic techniques of obtaining off-shell two-body scattering functions are described within this framework. Spin and angular momentum are treated in a covariant, kinematic-singularity free manner throughout these discussions. Finally, an example of the theory, which is preliminary to a more complete theory of the relativistic three-body problem, is presented. A single-particle exchange diagram is described, and an off-mass-shell three-body recoupling coefficient, which is similar to the on-shell three-body recoupling coefficient that was defined by Wick, is derived. 
INTRODUCTION

With the advent of many new medium-energy accelerators, an abundance of high-quality cross-section and polarization data for nuclear reactions at nonrelativistic and relativistic energies is becoming available? The theoretical understanding of these experiments requires an understanding of the relativistic many-body problem for strongly interacting particles with spin. Currently, the most popular thecretical approach to this problem is to assume that the target nucleus is composed of nucleons and higher-spin nucleon resonances (e.g., the $\Delta(1232)$ ) and that the projectile multiply scatters through the nuclear environment by interacting with these nucleons and resonances through the exchange of mesons? Many of these relativistic theories, however, are not applicable to particles with arbitrary spin (e.g., Refs. 3 and 4) or are unjustified generalizations of nonrelativistic scatering theory. For these reasons and because detailed theory of the strong force is still unarailable, it is desireable to develop a phenomenological, rela- 
tivistic, multiple-scattering theory that can deal with particles with arbitrary spin. This type of theory is the subject of this dissertation. (Note: Recentiy, there has been an attempt at formulating the nuclear reaction problem in terms of a more fundamental theory of the strong interaction. This theory, however, is still in its infancy and is nonrelativistic.)

The best known phenomenological many-body scattering theory is nonrelativistic multiple-scattering potential theory in which the two-body potential is the object of parameterization. This theory is a three-dimensional theory with one "off-shel1" variable. That is, the equations that arise in this theory (e.g., the LippmannSchwinger equation) consist of integral equations; the integrals range over the three-momenta of intermediate-state "particles"; and the scattering functions (or "vertex functions") that appear in these equations are dependent on one variable in addition to the three-momenta. In most cases, this additional variable is the total energy in the initial (or final) state; hence, potential theory is called an "off-unergy-shel1" scattering theory. Another aspect of potential theory, which is most appealing, is that it is based on the properties of the noninteracting multiple-particle Hilbert space of quantum mechanics? As such, the properties of that Hilbert space can be used to handle particles with spin, and standard angularmomentum techniques can be applied to simplify the problem. 
Potential theory, however, is not a relativistic theory, and most attempts to make it into a relativistic theory rely on unjustifiable procedures. For example, consider the Lorentz transformation to the center-of-mass reference frame of a two-body operator expectation value when the initial and final two-particle states have different total energies but equal total three-momentum. 10 (Note: This transformation is needed in any phenomenological theory that wishes to relate "off-shell" scattering amplitudes to "on-shell" data.) So, consider

$$
\left\langle\vec{k}_{1} \quad \vec{k}_{2}^{\prime}|V| \vec{k}_{1} \vec{k}_{2}\right\rangle \text { with } E^{\prime} \neq E \text { but } \vec{k}^{\prime}=\vec{k}
$$

where $E \equiv \varepsilon_{1}\left(\vec{k}_{1}\right)+\varepsilon_{2}\left(\vec{k}_{2}\right) \quad\left(\varepsilon_{1}(\vec{k})=\left(\vec{k}^{2}+m_{i}{ }^{2}\right)^{\frac{3}{2}}\right)$ and $\vec{k} \equiv \vec{k}_{1}+\vec{k}_{2}$. The velocity of the Lorentz transformation to the two-body centerof-mass in the initial state is $\vec{\beta}=\vec{K} / E$; whereas, in the final state the velocity is $\vec{\beta}^{\prime}=\vec{K} / E^{\prime}$. Hence, because $E^{\prime}$ is not equal to $E$, it appears that the center-of-mass reference frame can not be defined. In the nonrelativistic limit, however, $E^{\prime} \cong E \cong m_{1}+m_{2}$, which implies that $\vec{\beta}^{\prime} \cong \vec{\beta}$; hence, there is no ambiguity nonrelativistically. To overcome the ambiguity in the relativistic problem, some athors (e.g., Ref. 11) simply use the nonrelativistic velocity and neglect all relativistic effects that arise from the Lorentz transformation to the two-body center-of-mass reference frame. In other words, they assume that the operator expectation value can be evaluated in the center-of-mass refernce frame using the nonrelativistic 
relative momentum $\vec{p}$, where $\vec{p}=\left(m_{2} \vec{k}_{1}-m_{1} \vec{k}_{2}\right) /\left(m_{1}+m_{2}\right)$. A relativistic ralative momentum can, of course, be defined (see Eq. 3-5 and Ref. 12 and note that the correct relativistic definition of the relative momentum is not equivalent to replacing the masses by energies in the nonrelativistic expression). But, even if a relativistic expression for the relative momentum is used, there still exists the ambiguity as to the "correct" velocity to use in the Lorentz transformation to the two-body center-of-mass reference frame.

(Note: The above comments do not necessarily apply to the extensive research on the formulation of a relativistic potential theory from basic principles; but, the theory in this form has not yet been developed to a point where it is widely accepted in mediumenergy physics. Moreover, the relation between this type of theory and current theoretical-methods in high-energy physics - i.e., field theory - is not well understood (e.g., Ref. 9).)

Another off-energy-shell approach to the relativistic manybody problem is. Tytic S-matrix theory. 13-21 The advantage of this theory over relativistic potential theory is that it is formulated from on-shell unitarity relations, which implies that the properties of the on-shell scattering amplitudes and the relativistic multipleparticle Hilbert space can be used without modification (e.g., the problem of defining a center-of-mass reference frame is nonexistant in this theory - for example, see Ref. 22). Moreover, because the theory is based on on-shell information, the concepts of spin and angular momentum are well understood. 
Currently, however, the most poular theory of relativistic interactions is field theory. ${ }^{23-33}$ This is a completely relativistic theory and can be applied to the many-body problem; 32 but, several complications arise when it is used as a basis for a phenomenological treatment of the many-body problem with spin.

The first aspect of field theory that leads to a complexity greater than that found in the off-energy-shell theories is that field theory is a four-dimensional theory with many "off-shell" variables. That is, the equations that arise in field-theory (e.g., the Bethe-Salpeter equation ${ }^{21,25,33}$ ) consist of integral equations; the integrals range over the four-momenta of intermediate-state "particles"; and the n-point vertex functions that appear in these equations are dependent on $n$ variables in addition to the threemomenta. (n is the total number of incident and exit "particles" attached to the vertex.) These additional variables are associated with the 0 th (or energy) component of each momentum four-vector; hence, iield theory is called an "off-mass-shell" theory. The off-mass-shell vertex functions, which arise in field theory, are therefore dependent on more variables than the off-energy-shell vertex functions, which arise in potential theory or analytic s-matrix theory. This implies that field theory will, in general, be are complicated theory to deal with phenomenologically. (Note: The additional variables in field theory arise from its treatment of particles and antiparticles. In particular, the addi- 
tional degree of freedom that is obtained through the energy component of each momentum four-vector allows the theory to use the same vertex function to describe both particle and antiparticle scattering events. In the off-energy-shell theories, the distinction between particles and antiparticles is not so evident - see Chapter 4.)

Another aspect of field theory that differentiates it from the off-energy-shell theories is in its treatment of particles with spin. 25,27-30 In the off-energy-shell theories, it has been noted that the properties of the noninteracting, relativistic, multipleparticle Hilbert space can be used without modification, which implies that the spin properties of a particle with spin $s$ can be described using Hilbert-space state vectors that rotate as $2 s+1$ dimensional, irreducible representations of the rotation group SU(2) (see Chapters 1 and 2 and Ref. 41). In field theory, on the other hand, a field is constructed for each particle such that the field Lorentz transforms as an n-Jimensional representation of the Lorentz group $S I(2, C)$; and, $n$ is, in general, greater than $2 s+1$ (see sppendix $D$ and Ref. 28). Moreover, the off-mass-shell vertex function in field theory is defined by removing multicomponent "spinors" from the actual reaction amplitude, as in the ISZ reduction procedure, thereby complicating the appearence of both the vertex function and the propogators that appear in the theory. 
For example? 24 the spin-0 + spin-th interaction is described

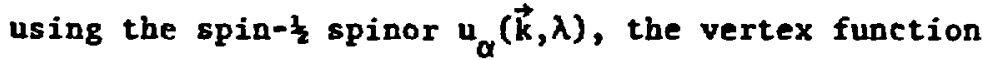

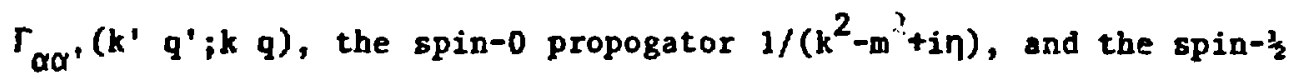
propogator $\left[(\gamma \cdot k+m) /\left(k^{2}-m^{2}+i \eta\right)\right]_{\alpha \alpha^{\prime}}$. The index $\alpha$ in these expressions is the "spinor index" ( $a=1,2,3$, or 4); $\lambda$ is the "spinprojection index" $(-s \leqq \lambda \leqq s)$; the vertex function and the propogators are functions of the off-mass-shell four-momenta (i.e., $\left.k_{0} \neq \varepsilon(\vec{k})\right)$; and, the spinor $u_{\alpha}(\vec{k} \lambda)$ is a function of the on-shell four-momentum (i.e., it is only defined when $k_{0}=\varepsilon(\vec{k})$ ). Feynman diagrams are constructed by combining the vertex functions with the propogators, integrating over intermediate state four-momenta, and summing over intermediate state spinor indices. The on-shell scattering amplitude is obtained by setting the "off-shell-energies" in the resultant vertex function equal to their on-shell values (i.e., $k_{0}+\varepsilon(\vec{k}), q_{0}+\varepsilon(\vec{q})$, etc.) and evaluating this on-shell vertex function between the appropriate spinors. In the off-energy-shell

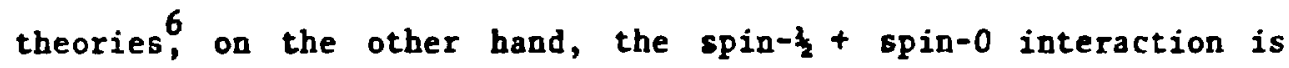
described using the vertex function $\left\langle\vec{k}^{\prime} \lambda^{\prime}, \vec{q}^{\prime}|T(E)| \vec{k} \lambda, \vec{q}^{\prime}\right.$ and the propogator $1 /(E-\varepsilon+i \eta)$, where $\varepsilon$ is a function of the threemomenta. Feynman diagrams are constructed by combining the vertex functions with the propogators, integrating over intermediate state three-nowenta, and suming over interwediate state syin-projection indices. The on-shell scattering amplitude is obtained by setting the "off-shell-energy" in the resultant vertex function equal to its 
on-shell value (i.e., equal to the appropriace function of the three-momenta).

This example illustrates the distinct difference between the spin and momentum-space structure of the equations in field theory and the corresponding structure in the off-energy-shell theories. This difference has, in fact, led to a greas deal of confusion regarding "off-mass-shell spinors" 3,4 (whicl,, by definition, do not exist) and the interpretation of the propogators as representations of particles and antiparticles in the intermediate states. ${ }^{12}$ It would be of benefit to many theories if the differences between the off-mass-shell theory and the off-energy-shell theories could be clarified. In particular, it would be desireable to develop a relativistic scattering theory with the following properties:

1) In its most fundamental form, the theory should be an offmass-shell theory that can be related to field theory.

2) Spin should be treated in its simplest form. That is, only $2 s+1$ spin-projection indices should be needed to describe the spin properties of a particle with spin s; spinors, with their inherent complexity, should not appear.

3) Particles with any spin should be described, and angularmomentum reduction techniques should be applicable.

4) The distinction between particles and antiparticles in the theory should be clear; so that, if the theory is related to an off-energy-shell theory, the decrease in the number of degrees of freedom will be well understood. 
A reduction of the theory to an equivalent off-energy-shell theory should be possible so that a phenomenology can be developed using a minimum number of free variables.

A theory that satisfies these requirements will be developed in this dissertation. This theory, os with any relativistic scattering theory, is formulated from the properties of the rotation and Lorentz groups and their application to noninteracting many-body systems in relativistic quantum mechanics. . $^{340,45-50}$ These aspects of the theory are discussed in Chapters 1 through 3 and in $\$ \$ 6.1$ and 6.2 .

For the results that are presented in Chapter 1 and Appendix B. I am indebted to J. D. Louck ${ }^{41}$ for an excellent explanation of the rotation groups. An essential aspect of his explazation is the distinction between the rotation groups $S O(3)$ and SU(2), which is manifest in the orthogonality relation for the rotation matrices (Eq. 1-26). This relation is stated incorrectly in several popular books on angular-momentum theory (e.g., Edmonds ${ }^{42}$ and Rose ${ }^{43}$ ). A generalization of Louck's method to the Lorentz groups, SO $(3,1)$ and SL $(2, C)$, is also presented in Chapter 1. The simplicity of this presentation is apparent when compared to other explanations of these groups and their representations (e.g., Refs. 35-38).

The application of these groups to the quantum-mechanical description of a single-particle system with spin is presented in Chapter 2. This theory was first developed by Wigner ${ }^{34}$ in 1939 , and, since that time, his work has become the theoretical foundation 
for most relativistic theories in quantum mechanics (including field theory). A number of texts and review articles that describe a variety of aspects of his theory do, of course, exist (e.g., Refs. 35-40 and 45-50); but, the fundamental aspects of that theory have not enjoyed a widespread appreciation in medium-energy physics. For this reason and to establish notation, Chapter 2 is devoted to a review of the aspects of Wigner's theory that have application to scattering theories at medium energies. Moreover, I have attempted to present the theory in a basis independent manner, so that the concepts on which it is founded will be apparent. In particular, Wigner's theory for a single-particle system is developed by first defining the fundamental state vector (i.e., the state vector that represents the particle at rest) and by defining the intrinsic spin of the particle through the rotational transformation properties of that state vector. The complementary set of Lorentz transformations is then defined, and a complete basis of state vectors for the single-particle Hilbert space is constructed. The $\hat{\mathbf{z}}^{45}$ and helicity $^{47}$ bases are used as examples of this general procedure. Finally, the Lorentz transformation and improper transformation properties of these two bases are described.

A completely parallel development for the noninteracting two-particle system is presented in Chapter 3 , and a number of bases for the two-particle Hilbert space are constructed. The chapter begins with a relativistic kinematic analysis of the two-particle 
system; a relative three-momentum is defined; and this relative momentum is shown to be equivalent to the "magic vector" of Aaron, et al. The two-particle Hilbert space is then defined, and bases for that space are constructed using the two-particle state vector in the center-of-mass reference frame as the fundamental state vector for the system. With this definition of the fundanental state vector, it is shown that the procedures of Chapter 2 can be used with little modification to develop the concept of angular momentum. A variety of state-vector transformations are also discussed.

The methods that are developed in Chapters 2 and 3 can also be applied to systems that contain an arbitrary number of particles (cf., $\$ \S 6.1$ and 6.2). In fact, this aspect of the method, which is rarely recognized in the literature, is one of its distinct advantages over other methods of deriving the same results (e.g., Ref. 37). In subsequent chapters, the concepts that are discussed in Chapters 2 and 3 are used to develop a theory of interacting particles.

With the basic description of noninteracting particles in relativistic quantum mechanics in hand, the question of interactions can be addressed in a theoretically meaningful way. Chapter 4 begins with a look at the on-shell (i.e., physical) scattering amplitude in a relativistic theory. The Poincaré and improper transformation properties of this amplitude are then considered; 
and, the improper transformation properties of the amplitude are used to suggest the existance of antiparticles. But, it is shown that if the antiparticle interpretation is to be correct, then a function, called an $M$ function, that is related to the scattering amplitude but is free of certain pathological behavior must be defined. This function is then used to develop an off-mass-shell scattering theory through the consideration of a time-ordered diagram. (Note: The use of time-ordered diagrams in this development was suggested by the work of M. B. Johnson, 52 which I have extended to include antiparticles and the notion of an off-mass-shell scattering event.)

The $M$ function that is used in this dissertation is similar to a function that was first defined by $S t a p{ }^{14}$ for use in analytic $S$ matrix theory. I, on the other hand, have used the $M$ function to develop an off-mass-shell scattering theory - the implication being that the $M$ function is, in fact, essentially equivalent to the vertex function that occurs in field theory. This supposition is verified in Appendix $D$, and an $M$ function for the $\pi N N$ vertex in the pseudoscalar interaction Hamiltonian model is explicitly constructed in $\$ 4.5$.

The $M$ function formalism that is presented in Chapter 4 has a number of very appealing properties. First, particles with arbitrary spin are included with no more difficulty than occurs for spin-t particles, and the spin-projection indices for a "particle" 
in the $M$ function correspond directly to the $2 s+1$ indices in the single-particle Hilbert space. Second, it is an off-mass-shell formalism, and the Lorentz transformation properties of the M function are well defined. Third, the formalism can be subjected to reduction techniques that allow one to construct an off-energy-shell scattering theory. Thus, the M-function formalism satisfies many of the requirements that were originally desired for a relativistic, phenomenological scattering theory.

The next step in the development of the scattering theory is to reduce the off-mass-shell formalism, which is presented in Chapter 4, to an equivalent off-energy-shell formalism. One can then consider a covariant phenomenology in the simplest possible terms. To this end, the relativistic two-body problem is discussed in Chapter 5. The discussion begins with a consideration of the on-shell scattering amplitude and progresses through the off-massshell M-function formalism. In the process, it is shown that an off-mass-shell "T function" (or $T$ matrix) can be defined and that this function can be interpreted as an off-mass-shell continuation of the scattering amplitude. The reduction procedure is then developed by applying the methods of Blankentecler and Sugar;3 but, in contrast to most applications of their formalism, 12,54 the reduction that is presented here relies on the concept of an off-mass-shell relative somentum. In the many-body problem, I expect that this procedure can be used to seperate subsystein interactions from their 
dependence on external quantities thereby yielding a seperable relativistic theory. 61 (For an example of the off-mass-shell relative momentum in the three-body problem see Chapter \%.) Covariant phenomenological methods that are based on the off-energy-shell formalism are also discussed.

In Chapter 6, which is the last chapter of this dissertation, I present an introductory look at the relativistic three-body problem through the consideration of a single-particle exchange diagram. The principal results of this chapter are a derivation of the off-mass-sheli relative momentum and a derivation of the threebody off-mass-shell recoupling coefficient. This coefficient is shown to be equivalent to Wick's three-body recoupling coefficient ${ }^{48}$ when all three particles are on-she1l; but, in any off-shell environment, this coefficient deviates from Wick's definition. This deviation occurs in the off-mass-shell theory and in the off-energyshell theory. Work on the complete relativistic three-body problem is currently in progress.

The net goals of this dissertation have thus been achieved. In particular, this work details the use of spin and angular momentum in an off-mass-shell, relativistic, scattering theory; and, the reduction of this theory to an equivalent off-energy-shell theory is described. Moreover, an example has been given that will serve as the starting point for a complete relativistic description of the three-body problem. 


\section{Chapter 1}

THE ROTATION AND LORENTZ GROUPS

Since 1939, when Wigner first established the connection between the representations of the Lorentz group and guantum mechanics, this group has acquired a pre-eminent position in theoretical physics. A knowledge of the Lorentz group is thus essential to an understanding of relativistic scattering theories.

The simplest group to be considered in this chapter is the rotation group SO(3), which is used to represent rotations in 3 real three-dimensional vector space. An extension of this group to a conplex two-dimensional space is then defined by associating a complex $2 \times 2$ matrix with each vector in the three-dimensional space. Each element of $S O(3)$ is thereby mapped onto two unitary unimodular matrices in the two-dimensional space, and these matrices are classified as representations of the group SU(2). This procedure is detailed in $\$ \S 1.1$ and 1.2 . 
The concept of a two-component vector (or spinor) is also established by this procedure. This vector is used to represent spin- $\frac{1}{2}$ particles, and it is shown that this vector has the following peculiar property: It does not return to itself when rotated by an angle of $2 \pi$ about any axis. This aspect of the group $\mathrm{SU}(2)$, which is assumed to be the correct group for describing rotations in quantum mechanics, implies the orthogonality relation that is given in Eq. 1-26, which is incorrectly stated in many texts on angularmomentum theory (e.g., Edmonds ${ }^{42}$ and Rose $\left.{ }^{43}\right)$. The higher-dimensional representations of the group SU(2) are discussed in $\$ \S 1.3$ and 1.4 .

The Lorentz groups, $S O(3,1)$ and $S L(2, C)$, are then developed in exact analogy with the development of the rotation groups, and it is shown that the higher-dimensional representations of the groups SL $(2, C)$ and sU(2) are essentially identical. This aspect of the group representations is used throughout this dissertation.

A detailed derivation of the relation between the groups So(3) and SU(2), which is easily extended to the Lorentz groups, has been relegated to Appendix B. Refs. 15, 26, 41, and 45 have been used extensively in the preparation of this chapter and Chapter 2. 


\subsection{Rotations: SO(3) 41}

Consider the rotation of a vector $\vec{x}$ about the unit vector $\hat{n}$ $(\hat{n} \cdot \hat{\mathbf{n}}=1)$ by an angle $\phi(0 \leq \phi \leq \pi)$.

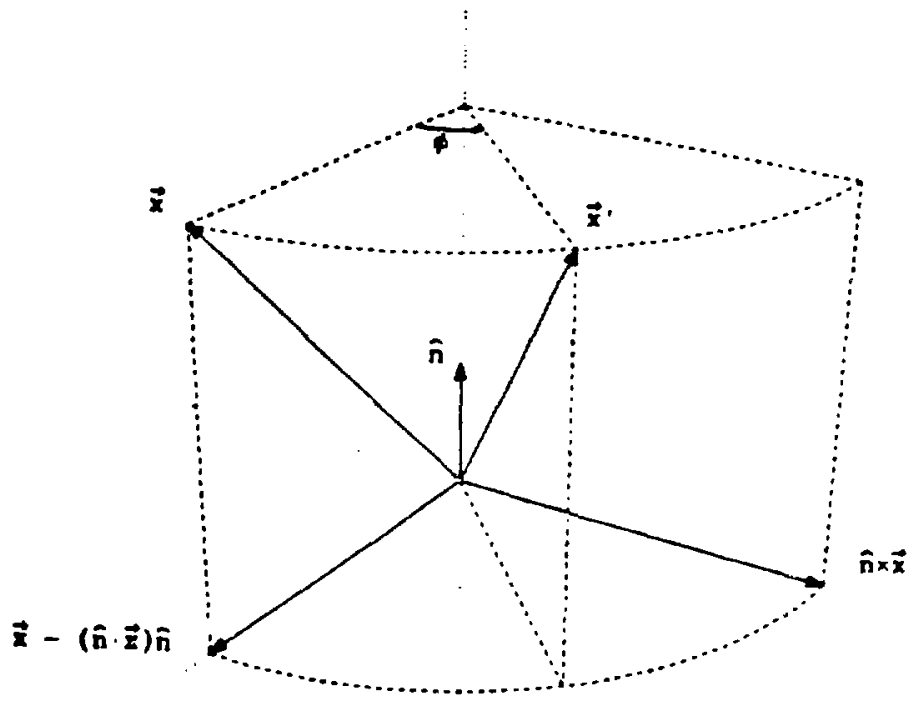

Fig. 1.1 Rotation of a vector $R(\phi, \hat{n}): \vec{x}$.

Fig. 1.1 shows that the vector $\vec{x}$ is transformed into a new vector $\vec{x}$, under the action of this rotation. In other words,

$$
Q(\phi, \hat{n}): \vec{x}+\vec{x} \cdot=(\hat{a} \cdot \vec{x}) \hat{n}+(\vec{x}-(\hat{n} \cdot \vec{x}) \hat{a}) \cos \phi+(\hat{n} \times \vec{x}) \sin \phi \quad(1-1)
$$


If $\overrightarrow{\mathrm{x}}$ is written as a column vector,

$$
\vec{x}=\left(\begin{array}{l}
x_{1} \\
x_{2} \\
x_{3}
\end{array}\right),
$$

then the action of the rotation, $R(\phi, \hat{n})$, can be represented by a $3 \times 3$ matrix, $r(\phi, \hat{n})$.

$$
\text { R. }(\phi, \hat{n}): \vec{x} \rightarrow \vec{x}^{\prime}=r(\phi, \hat{n}) \vec{x}
$$

The elements of $r(\phi, \hat{n})$ are (cf., Eq. 1-1)

$$
r(\phi, \hat{n})_{i j}=\delta_{i j} \cos \phi+n_{i} n_{j}(1-\cos \phi)+\varepsilon_{i k j} n_{k} \sin \phi: i, j=1,2,3
$$

$\delta_{i j}$ is the Kronecker-delta, $\varepsilon_{i k j}$ is the Levi-Civita antisymmetric tensor, and a sum over repeated indices is implied, $r(\phi, \hat{n})$ is a real, orthogonal $\left(r_{r}^{T}=1\right)$, proper (det $r=1$ ) $3 \times 3$ matrix. Hence, the matrix $r$ is a representation of the group So(3) (Special Orthogonal group in three dimensions - special because det $r=1$ ).

1.2 Rotations: $\operatorname{su(2)^{41}}$

The Pauli matrices are defined as

$\sigma_{0}=\left(\begin{array}{ll}1 & 0 \\ 0 & 1\end{array}\right) \quad \sigma_{1}=\left(\begin{array}{ll}0 & 1 \\ 1 & 0\end{array}\right) \quad \sigma_{2}=\left(\begin{array}{cc}0 & -i \\ i & 0\end{array}\right) \quad \sigma_{3}=\left(\begin{array}{cc}1 & 0 \\ 0 & -1\end{array}\right)$ 
These matrices form a complete, linearly independent set of $2 \times 2$ hermitian $\left(\sigma_{\mu}^{\dagger}=\sigma_{\mu}\right)$ matrices. Other properties of the Pauli matrices are:

i) The trace of $\sigma_{i}$ is zero $\left(\operatorname{tr} \sigma_{i}=0\right)$

$$
\left[\sigma_{i}, \sigma_{j}\right] \equiv \sigma_{i} \sigma_{j}-\sigma_{j} \sigma_{i}=2 i \varepsilon_{i j k} \sigma_{k}
$$

where $i, j$, and $k$ are equal to 1,2 , or 3 .

The Pauli spin matrices allow one to associate a $2 \times 2$ matrix with any vector $\vec{x}$. This matrix is defined by the relation

$$
\vec{x} \leftrightarrow-\vec{x} \cdot \vec{\sigma}=\left(\begin{array}{rc}
-x_{3} & -x_{1}+i x_{2} \\
-x_{1}-i x_{2} & x_{3}
\end{array}\right)
$$

where the minus sign has been included for later convenience ( $f$., Eq. 1-38). The $2 \times 2$ matrix, $\vec{x} \cdot \vec{\sigma}$, is hermitian, traceless, and has determinant equal to $\vec{x}^{2}$ (iet $\vec{x} \cdot \overrightarrow{0}=\vec{x} \cdot \vec{x}$ ).

The properties of hetmiticity and determinant are preserved under the similarity transformation

$$
\vec{x} \cdot \vec{\sigma} \rightarrow \vec{x}^{\prime} \cdot \vec{\sigma}=A \vec{x} \cdot \vec{\sigma} A^{\dagger}
$$

when $A$ is any unimodular (det $A=1$ ) $2 \times 2$ matrix. If $A$ is unitary $\left(A A^{\dagger}=A^{\dagger} A=1\right)$ the trace of $\vec{x} \cdot \vec{\sigma}$ is also preserved. It is therefore expected that the set of unitary similarity transformations on the atrix $\vec{x} \cdot \vec{\sigma}$ is closely related to the set of rotations on the vector 
$\vec{x}$. In fact, it is shown in Appendix B that a given $2 \times 2$ unitary matrix, A, determines a unique $3 \times 3$ real, orthogonal, proper matrix, $r$, where $r$ is defined by $\vec{x}^{\prime} \equiv \vec{r} \vec{x}$ in Eq. 1-9. It is also shown that if $A_{1}$ determines $r_{1}$ and $A_{2}$ determines $r_{2}$ then $A_{1} A_{2}$ determines $r_{1} r_{2}$. Thus, $r$ is a rotation (representation of $s(3)$ ). In terms of $A$ the elements of $r$ are given by

$$
r_{i j}=\frac{1}{2} \operatorname{tr}\left(\sigma_{i} A \sigma_{j} A^{\dagger}\right): i, j=1,2,3
$$

Conversely, Eq. 1-10 can be inverted to determine the $2 \times 2$ matrix, A, from a given $3 \times 3$ rotation matrix, $r$. It is shown in Appendix $B$ that there exist just two unitary and unimodular matrices, $A$, that map onto the same $r(\phi, \hat{n})$. These are

$$
A=R(\phi, \hat{n}) \equiv \sigma_{0} \cos \frac{\phi}{2}-i \hat{n} \cdot \vec{\sigma} \sin \frac{\phi}{2}
$$

and

$$
A=-R(\phi, \hat{n})=R(2 \pi-\phi,-\hat{n}) \quad: 0 \leqq \phi \leqq \pi, \hat{n} \cdot \hat{\mathbf{n}}=1 .
$$

Thus, the group SU(2) (Special Unitary group in two dimensions) maps 2:1 onto the group SO(3). 


\subsection{Paramaterizations of the Rotation Groups ${ }^{41}$}

\section{$1.3 .1(\phi, \hat{n})$ Paramaterization}

In $\$ 1.1$, it was shown that a rotation can be paramaterized by specifying a rotation angle, $\phi$, and an axis of rotation, $\hat{n}$. The domain of these parameters is $0 \leqq \phi \leqq \pi$ and $\hat{n} \cdot \hat{n}=1$. Eq. $1-11$ show's that there are two $2 \times 2$ unitary unimodular matrices, $R(\phi, \hat{n})$ and $-R(\phi, \hat{n})$, that map onto a given $3 \times 3$ real, orthogonal, proper matrix, $\mathbf{r}(\phi, \hat{n})$. This may be restated by saying that the set of $2 \times 2$ matrices

$$
R(\phi, \hat{n})=\sigma_{0} \cos \frac{\phi}{2}-i \hat{n} \cdot \vec{\sigma} \sin \frac{\phi}{2}=e^{-i \hat{n} \cdot \sigma(\phi / 2)}
$$

defined in the domain $0 \leqq \phi \leqq 2 \pi$ and $\hat{\mathbf{n}} \cdot \hat{\mathbf{n}}=1$ map $2: 1$ onto $r(\phi, \hat{\mathbf{n}})$.

It is clear from this paramaterization that the group space of $\mathrm{SU}(2)$ is larger than the group space of $S O(3)$. It can also be seen that the group space of $\mathrm{SU}(2)$ can be associated with points on a unit sphere in a four-dimensional space. This space is defined by

$$
R=\alpha_{0} \sigma_{0}-i \vec{a} \cdot \vec{\sigma}
$$

with $\quad \alpha_{0}{ }^{2}+\vec{\alpha}^{2}=1$. 


\subsubsection{Euler-Rodriguez Paramaterization}

This paramaterization uses $E_{q} \cdot 1-13$ and the relation $r_{i j}=$ $\frac{1}{2} \operatorname{tr}\left(\sigma_{i} R \sigma_{j} R^{\dagger}\right)$ (Eq. 1-10) to explicitly display the $2: 1$ mapping of su(2) onto so(3). One obtains

$$
r=\left(\begin{array}{ccc}
\alpha_{0}^{2}+\alpha_{1}^{2}-\alpha_{2}^{2}-\alpha_{3}^{2} & 2 \alpha_{1} \alpha_{2}-2 \alpha_{0} \alpha_{3} & 2 \alpha_{1} \alpha_{3}+2 \alpha_{0} \alpha_{2} \\
2 \alpha_{1} \alpha_{2}+2 \alpha_{0} \alpha_{3} & \alpha_{0}^{2}+\alpha_{2}^{2}-\alpha_{3}^{2}-\alpha_{1}^{2} & 2 \alpha_{2} \alpha_{3}-\alpha_{0} \alpha_{1} \\
2 \alpha_{1} \alpha_{3}-2 \alpha_{0} \alpha_{2} & 2 \alpha_{2} \alpha_{3}+2 \alpha_{0} \alpha_{1} & \alpha_{0}^{2}+\alpha_{3}^{2}-\alpha_{1}^{2}-\alpha_{2}^{2}
\end{array}\right)
$$

It is evident that $R\left(\alpha_{0}, \vec{\alpha}\right)$ and $R\left(-\alpha_{0},-\vec{\alpha}\right)$ map onto the same $r$

\subsubsection{Euler Angle Paramaterization}

The Euler angles are defined by the following set of rotations:

$r_{1}$ : rotate by the angle $\alpha$ about the vector $\hat{\mathbf{n}}_{1}=(0,0,1)$

$r_{2}$ : rotate by the angle $\beta$ about the vector $\hat{n}_{2}=(-\sin \alpha, \cos \alpha, 0)$

$r_{3}$ : rotate by the angle $\gamma$ about the vector $\hat{n}_{3}=(\cos \alpha \sin \beta$, $\sin (\alpha \sin \beta, \cos \beta)$

Where $0 \leqq \alpha \leqq 2 \pi, 0 \leqq \beta \leqq \pi, 0 \leqq \gamma \leqq 2 \pi$ and an orthogonal set of 
coordinate axes, $\hat{x}=(1,0,0), \hat{y}=(0,1,0), \hat{z}=(0,0,1)$, has been established. The rotation $x(\alpha, \beta, \gamma)=r_{1} r_{2} r_{3}$ covers the group space of so(3) exactly once.

To interpret these rotations actively, one can refer to a fixed set of coordinate axes and employ the following result:

$$
R(\phi, \hat{n})=S^{-1} R(\phi, s \hat{n}) S
$$

Where, $S$ is an arbitrary rotation. (To see that this is true, consider the following sequence: (1) Bring the vector $\hat{n}$ into coincidence with the vector $\hat{\mathbf{n}}^{\prime} \equiv \mathbf{s} \hat{\mathrm{n}}$. (2) Rotate by $\phi$ about $\hat{\mathbf{n}}^{\prime}$. Retura the vector $\hat{n}^{\prime}$ to $\hat{\mathbf{n}}$.) This result can be used to show that

$$
R_{n} R_{n-1} \cdots R_{2} R_{1}=R_{1} R_{2}^{\prime} \cdots R_{n-1}^{\prime} R_{n}^{\prime}
$$

Where, $R_{k}$ is a rotation by the angle $\phi_{k}$ about the vector $\hat{n}_{k}, R_{k}^{\prime}$ is a rotation by the angle $\phi_{k}$ about the vector $\hat{n}_{k}^{\prime} \equiv\left(r_{k-l} \cdots r_{1}\right)^{-1} \hat{n}_{k}$ and $\hat{\mathrm{n}}_{1} \equiv \hat{\mathbf{n}}_{1}$.

Applying Eq. 1-16 to the Euler angle paramaterization,

$$
r(\alpha, \beta, \gamma)=r\left(\gamma, \hat{n}_{3}\right) r\left(\beta, \hat{n}_{2}\right) r\left(\alpha, \hat{n}_{1}\right)
$$

where $\quad \hat{\mathbf{n}}_{1}=\hat{\mathbf{z}}$

$$
\begin{aligned}
& \hat{\mathbf{n}}_{2}=r\left(\alpha, \mathrm{n}_{1}\right) \hat{\mathrm{y}} \\
& \hat{\mathbf{n}}_{3}=r\left(\beta, \hat{\mathbf{n}}_{2}\right) \mathbf{r}\left(\alpha, \hat{\mathrm{n}}_{1}\right) \hat{\mathbf{z}}
\end{aligned}
$$


yields $\quad \hat{\mathbf{n}}_{1}^{\prime}=\hat{\mathbf{n}}_{1}=\hat{\mathbf{z}}$

$$
\begin{aligned}
& \hat{n}_{2}^{\prime}=r\left(\alpha, \hat{n}_{1}\right)^{-1} \hat{n}_{2}=\hat{y} \\
& \hat{n}_{3}^{\prime}=\left(r\left(\beta, \hat{n}_{2}\right) r\left(\alpha, \hat{n}_{1}\right)\right)^{-1} \hat{n}_{3}=\hat{z} .
\end{aligned}
$$

Hence, $\quad r(\alpha, \beta, \gamma)=r(\alpha, \hat{z}) r(\beta, \hat{y}) r(\gamma, \hat{z})$.

$$
=\left(\begin{array}{ccc}
\cos \alpha & -\sin \alpha & 0 \\
\sin \alpha & \cos \alpha & 0 \\
0 & 0 & 1
\end{array}\right)\left(\begin{array}{ccc}
\cos \beta & 0 & \sin \beta \\
0 & 1 & 0 \\
-\sin \beta & 0 & \cos \beta
\end{array}\right)\left(\begin{array}{ccc}
\cos \gamma & -\sin \gamma & 0 \\
\sin \gamma & \cos \gamma & 0 \\
0 & 0 & 1
\end{array}\right)
$$

The two $2 \times 2$ matrices that map onto this rotation are (see Eq. 1-12)

$$
\begin{aligned}
& R(\alpha, \beta, \gamma)=e^{-i \frac{\alpha}{2} \sigma_{3}} e^{-i \frac{\beta}{2} \sigma_{2}} e^{-i \frac{\gamma}{2} \sigma_{3}} \\
& =\left(\begin{array}{cc}
e^{-i \frac{\alpha}{2}} \cos \frac{\beta}{2} e^{-i \frac{\gamma}{2}} & -e^{-i \frac{\alpha}{2}} \sin \frac{\beta}{2} e^{i \frac{\gamma}{2}} \\
e^{i \frac{\alpha}{2}} \cos \frac{\beta}{2} e^{-i \frac{\gamma}{2}} & e^{i \frac{\alpha}{2}} \sin \frac{\beta}{2} e^{i \frac{\gamma}{2}}
\end{array}\right) \\
& \text { and } \quad-R(\alpha, \beta, \gamma)=R(\alpha, \beta+2 \pi, \gamma) \text {. }
\end{aligned}
$$

Thus, the set of matrices $R(\alpha, \beta, \gamma)(0 \leqq \alpha \leqq 2 \pi, 0 \leqq \beta \leqq \pi$ and $2 \pi \leqq \beta \leqq 3 \pi, \quad 0 \leqq \gamma \leqq 2 \pi)$ map $2: 1$ onto $r(\alpha, \beta, \gamma)$. Note that the covering space for SU(2) includes the domain $2 \pi \leqq \beta \leqq 3 \pi$ and note that $R(\alpha, \beta, \gamma) \neq R(\alpha, \beta+2 \pi, \gamma)$. 


\subsection{Higher-Dimensional Representations of SU(2) 41,45}

In analogy with the action of the matrix $r$ on the three-

component vector $\vec{x}$, the matrix $R$ can be considered as acting on a two-component vector, called a two-component spinor. A spin-1/2 particle is an object that transforms under rotations as a twocomponent spinor. The $2 \times 2$ rotation matrix for this object is defined as $D^{\frac{1}{2}}(R) \equiv R$. Particles with higher spin (i.e., $1, \frac{3}{2}, \ldots$ ) are defined to be objects that transform under rotations via higherdimensional irreducible representations of $\mathrm{SU}(2)$. (A set of representation matrices is irreducible if the only matrices that comnute with all the matrices of the set are constant multiples of the identity.)

These higher-dimensional representations of $\mathrm{SU}(2)$ can be obtained by considering a rotation of the $2 s+1$ component spinor whose elements are

$$
\xi_{m}^{s}(a, b)=\frac{a^{s+m} b^{s-m}}{[(s+m) !(s-m) !]^{\frac{1}{2}}} \quad:-s \leqq m \leqq s \quad(1-20)
$$

where $\xi^{\frac{1}{2}} \equiv\left(\begin{array}{l}a \\ b\end{array}\right)$ is the aforementioned two-component spinor. Under a rotation,

$$
R=\left(\begin{array}{ll}
R_{11} & R_{12} \\
R_{21} & R_{22}
\end{array}\right)
$$


the spinor $\xi^{s}$ becomes

$$
\begin{aligned}
\xi_{m^{s}}^{s}\left(a^{\prime}, b^{\prime}\right)= & \sum_{m^{\prime} n}\left(\begin{array}{c}
s+m \\
n
\end{array}\right)\left(\begin{array}{c}
s^{\prime}-m^{\prime} \\
m^{\prime}-m+n
\end{array}\right) R_{11}^{s+m-n} R_{12}^{n} R_{21}^{m^{\prime}-m+n} \\
& \times R_{22}^{s-m^{\prime}-n} a^{s+m^{\prime}} b^{s-m^{\prime}}
\end{aligned}
$$

where $\left(\begin{array}{l}m \\ n\end{array}\right)$ is a binomial coefficient and the sums are carried out over all terms for which the factorials are nonnegative.

The $2 s+1$ dimensional rotation matrix is defined by

$$
\xi^{s}\left(a^{\prime}, b^{\prime}\right)=D^{s}(R) \xi^{s}(a, b)
$$

and the elements of $D^{S}(R)$ are determined from Eq. $1-22$.

$$
\begin{aligned}
D_{m^{\prime} m}^{s}(R)=\sum_{n} & \frac{\left[\left(s+m^{\prime}\right) !\left(s-m^{\prime}\right) !(s+m) !(s-m) !\right]^{\frac{1}{2}}}{\left(s+m^{\prime}-n\right) ! n !\left(m-m^{\prime}+n\right) !(s-m-n) !} \\
& \times R_{11}^{s+m^{\prime}-n} R_{12}^{n} R_{21}^{m-m^{\prime}+n} R_{22}^{s-m-n}
\end{aligned}
$$

The rotation matrices, $D^{S}(R)$, have the following properties ${ }^{45}$

i) They are representations of $S U(2)\left(D^{S}\left(R_{1}\right) D^{S}\left(R_{2}\right)=D^{S}\left(R_{1} R_{2}\right)\right)$.

ii) They are unitary $\left(D^{S}(R)^{\dagger}=D^{S}(R)\right)$.

iii) They form an irreducible set.

iv) No other representations of $\mathrm{SU}(2)$ have these properties, except a set $D(R)^{\prime}=U D(R) U^{\dagger}$ with $U$ unitary. 
In terms of the Euler angles (\$1.3.3), the rotation matrices are

$$
\begin{aligned}
D_{m^{\prime} m}^{s}(R(\alpha, \beta, \gamma)) & =D_{m^{\prime} m}^{s}(R(\alpha, \hat{z}) R(\beta, \hat{y}) R(\gamma, \hat{z})) \\
& =e^{-i m^{\prime} \alpha} d_{m^{\prime} m}^{s}(\beta) e^{-i m \gamma}
\end{aligned}
$$

where $d_{m^{\prime} m}^{s}(\beta) \equiv D_{m^{\prime} m}^{s}(R(\beta, \hat{y}))$

\subsubsection{Crthogonality of $D^{S}(R)$}

The rotation victrices, $D^{S}(R)$, form a complete set of functions over the unit sphere in the four-dimensicnal space of $\operatorname{su(2)}$, and it can be shown ${ }^{41}$ that these functions satisfy the following orthogonality relation:

$$
\begin{aligned}
& \int_{0}^{2 \pi} d \alpha \int_{0}^{2 \pi} d \gamma \int_{0}^{\pi} \sin \beta d \beta D_{m^{\prime} m}^{s^{\prime}}(R(\alpha, \beta, \gamma)) D_{\mu^{\prime} \mu}^{s}(R(\alpha, \beta, \gamma))^{*} \\
& +\int_{0}^{2 \pi} d \alpha \int_{0}^{2 \pi} d \gamma \int_{2 \pi}^{3 \pi} \sin \beta d \beta D_{m^{\prime} m}^{s^{\prime}}(R(\alpha, \beta, \gamma)) D_{\mu^{\prime} \mu^{s}}^{s}(R(\alpha, \beta, \gamma))^{*} \\
& =\frac{16 \pi^{2}}{2 s^{2}} \delta_{s^{\prime} s} \delta_{m^{\prime} \mu^{\prime}}, \delta_{m \mu}
\end{aligned}
$$

(Note: The integrations in this equation must be carried out over the entire covering space of $\mathrm{SU}(2)$. This aspect of the orthogonality relation has not always been recognized in the literature. ${ }^{42,43}$; 
In later chapters, Eq. 1-26 will be used to expand angular functions in terms of the complete set of rotation matrices. The following results will then be useful:

i)

$$
\begin{aligned}
& \text { If } m^{\prime}=\mu^{\prime} \text { in Eq. 1-26, then } \\
& \begin{aligned}
\int_{0}^{2 \pi} \mathrm{d} \alpha \int_{0}^{2 \pi} \mathrm{d} \gamma \int_{0}^{\pi} \sin \beta \mathrm{d} \beta \mathrm{D}_{\mathrm{m}^{\prime} \mathrm{m}}^{\mathrm{s}^{\prime}}(\mathrm{R}(\alpha, \beta, \gamma)) \mathrm{D}_{\mathrm{m}^{\prime} \mu^{\mathrm{s}}}(\mathrm{R}(\alpha, \beta, \gamma))^{+} \\
\quad=\frac{8 \pi^{2}}{2 \mathrm{~s}+1} \delta_{\mathrm{s}^{\prime} s} \delta_{\mathrm{m} \mu}
\end{aligned}
\end{aligned}
$$

The same reduction occurs if $n=\mu\left(m^{\prime} \neq \mu^{\prime}\right)$.

ii) Given a function of three independent angles, one can expand that function in terms of the rotation matrices.

$$
f(\alpha, \beta, \gamma)=\sum_{j m^{\prime} m} D_{m^{\prime} m}^{j}(R(\alpha, \beta, \gamma)) f_{m^{\prime} m}^{j}
$$

iii) Given a function of two independent angles, one can define $g(\alpha, \beta, \gamma) \equiv e^{-i \gamma \mu} f(\alpha, \beta)$ with $\mu$ arbitrary. Then

$$
\begin{aligned}
f(\alpha, \beta) & =\frac{1}{2 \pi} \int_{0}^{2 \pi} d \gamma e^{i \gamma \mu} \underset{j m^{\prime} m^{\prime} m^{j}}{D_{m}^{j}}(R(\alpha, \beta, \gamma)) g_{m^{\prime} m}^{j} \\
& =\sum_{j m^{\prime}} D_{m^{\prime} \mu^{j}}^{j}(R(\alpha, \hat{z}) R(\beta, \hat{y})) f_{m^{\prime}}^{j}
\end{aligned}
$$

where $\mu$ is still arbitrary. 
iv) Following the same procedure, a function of just one angle can be expanded as

$$
f(\beta)=\sum_{j} d_{\mu}^{j}{ }^{j}(\beta) f^{j}
$$

where both $\mu^{\prime}$ and $\mu$ are arbitrary.

\subsection{Lorentz Transformations: $\mathrm{SO}(3,1)$}

A four-vector, $x$, is defined to be the concatenation of a temporal component, $x_{0}$, and a three-vector, $\vec{x}$. Under the action of a Lorentz boost by velocity $\vec{\beta}(0 \leqq|\vec{\beta}| \leqq 1)$, the vector $x$ is transformed into a new vertor $x^{\prime}$, whose components are 58

$$
\begin{aligned}
& x_{0}^{\prime}=\gamma\left(x_{0}-\vec{\beta} \cdot \vec{x}\right) \quad: \gamma \equiv\left(1-\vec{\beta}^{2}\right)^{-\frac{1}{2}} \\
& \vec{x}^{\prime}=\vec{x}+\gamma \vec{\beta}\left(\frac{\gamma}{\gamma+1} \vec{\beta} \cdot \vec{x}-x_{0}\right)
\end{aligned}
$$

This result can be rewritten by defining a boost parameter $x \equiv$ $\tanh ^{-1}(|\vec{\beta}|)$ and by denoting the direction of the boost by the unit vector $\hat{n} \equiv \vec{\beta} /|\vec{\beta}|$. With this paramaterization,

$$
\mathcal{L}(x, \hat{\mathrm{n}}): \begin{aligned}
& \mathrm{x}_{0} \rightarrow \mathrm{x}_{0}^{\prime}=\mathrm{x}_{0} \cosh \chi-\hat{\mathrm{n}} \cdot \overrightarrow{\mathrm{x}} \sinh \chi \\
& \overrightarrow{\mathrm{x}} \rightarrow \vec{x}^{\prime}=\vec{x}-\hat{\mathbf{n}}(\hat{\mathbf{n}} \cdot \overrightarrow{\mathrm{x}})(1-\cosh \chi)-\hat{\mathrm{n}} \mathrm{x}_{0} \sinh \chi
\end{aligned}
$$

with $\cosh \chi=\gamma$ and $\sinh \chi=\gamma|\vec{\beta}|$. 
If $x$ is written as a column vector,

$$
x \equiv\left(\begin{array}{l}
x_{0} \\
x_{1} \\
x_{2} \\
x_{3}
\end{array}\right)
$$

then the action of the Lorentz boost, $\mathcal{L}(\chi, \hat{n})$, can be represented by a $4 \times 4$ matrix, $\ell(X, \hat{n})$.

$$
\mathcal{L}(x, \hat{n}): x \rightarrow x^{\prime}=\mathcal{L}(x, \hat{n}) x
$$

The elements of $\ell(x, \hat{n})$ are (cf., Eq. 1-32)

$$
\begin{aligned}
& \ell(x, \hat{n})_{00}=\cosh x \\
& \ell(x, \hat{n})_{0 i}=\ell(x, \hat{n})_{i 0}=-n_{i} \sinh x \quad: i=1,2,3 \\
& \ell(x, \hat{n})_{i j}=\delta_{i j}-n_{i} n_{j}(1-\cosh X) \quad: \quad i, j=1,2,3
\end{aligned}
$$

The scalar product of two four-vectors is defined as

$$
x \cdot y=x_{0} y_{0}-\vec{x} \cdot \vec{y} \equiv x^{T} g y
$$

where, $\quad 8_{00}=1,8_{i i}=-1$, and $8_{\mu \nu}=0$ if $\mu \neq v$.

The scalar product, $x \cdot y$, is invariant under the action of a Lorentz boost (i.e., $\ell x \cdot \ell y=x \cdot y)$. This alone implies that 


$$
\ell^{T} \& \ell=B
$$

which furtber implies that det $\ell= \pm 1 \quad\left(\operatorname{det} \ell^{T} g \ell=-(\operatorname{det} \ell)^{2}=\right.$ det $g=-1)$ and $\ell_{00} \geqq+1$ or $\ell_{00} \leqq-1\left(\left(\ell_{00}\right)^{2}-\sum_{1}\left(\ell_{i 0}\right)^{2}=1\right)$. Thus, in addition to the Lorentz boosts that have det $\ell=+1$ and $\ell_{00}$ $\geqq+1$, there exist three other categories of Lorentz transformations that leave the scalar product invariant. These are listed as categories II through IV in Table 1.1.

$\begin{array}{lcl} & \operatorname{det} \ell & \ell_{00} \\ \text { I } & +1 & \geqq+1 \\ \text { II } & -1 & \geqq 1 \\ \text { III } & -1 & \leqq-1 \\ \text { IV } & +1 & \leqq-1\end{array}$

Table 1.1 The Lorentz group

Lorentz transformations that belong to category I form the proper (or orthochronous) Lorentz group, SO(3,1) (Special Orthogonal group in 3 plus 1 dimensions - 3 plus 1 because of the scalar product, Eq. 1-35). This group is composed of all Lorentz boosts, $\ell(\chi, \hat{n})$, and rotations, $r(\phi, \hat{n}) . \quad(r(\phi, \hat{n})$ can be taken to be a $4 \times 4$ matrix that leaves the $x_{0}$ component of $x$ unchanged.) Lorentz transformations that belong to categories II through IV can be obtained from the Lorentz transformations of category $I$ via the improper uperations of space inversion and time reversal. These operations are defined by the following relations: 


$$
\begin{aligned}
& I \rightarrow I I: I_{s} \ell \\
& I \rightarrow I I I: I_{t} \ell \\
& I \rightarrow I V: I_{s} I_{t} \ell
\end{aligned}
$$

with $I_{s}=8$ and $I_{t}=-8$.

\subsection{Lorentz Transformations: SI $(2, C)$}

In exact analogy with the treatment of $\operatorname{SU}(2)(\$ 1.2)$, a $2 \times 2$ matrix can be associated with any four-vector $x$. This matrix is defined by

$$
x \leftrightarrow x \cdot \sigma=\left(\begin{array}{cc}
x_{0}-x_{3} & -x_{1}+i x_{2} \\
-x_{1}-i x_{2} & x_{0}+x_{3}
\end{array}\right)
$$

The $2 \times 2$ matrix, $x \cdot \sigma$, is hermitian and has determinant equal to $x^{2}$ $(\operatorname{det} x \cdot \sigma=x \cdot x)$.

The properties of hermiticity and determinant are preserved under the similarity transformation

$$
x \cdot \sigma \rightarrow x^{\prime} \cdot \sigma=A x \cdot \sigma A^{\dagger}
$$

when $A$ is any unimodular (det $A=1) 2 \times 2$ matrix. And, as in the rotation groups, it is expected that the set of unimodular similarity transformations on the matrix $x \cdot \sigma$ is closely related to the set of Lorentz transformations on the vector $x$. In fact, it can be 
shown using the methods of Appendix B that a given $2 \times 2$ unimodular matrix, $A$, determines a unique $4 \times 4$ real, orthogonal $\left(\ell^{T} g \ell=8\right)$, proper matrix, $\ell$, where $\ell$ is defined by $x^{\prime} \equiv \ell x$ in Eq. 1-39. Furthermore, if $A_{1}$ determines $\ell_{2}$ and $A_{2}$ determines $\ell_{2}$ then $A_{1} A_{2}$ determines $\ell_{1} \ell_{2}$. Thus, $\ell$ is a proper Lorentz transformation (representation of $S(3,1)$ ). In terms of $A$, the elements of $\mathcal{L}$ are given by

$$
l_{\mu v}=\frac{1}{2} \operatorname{tr}\left(\tilde{\sigma}_{\mu} \tilde{\sigma}_{v}{ }^{\dagger}\right)
$$

where $\tilde{\sigma} \equiv\left(\sigma_{0},-\vec{\sigma}\right)$.

Conversely, Eq. 1-40 can be inverted to obtain two unimodular matrices, $A$, that map onto the same $\ell(X, \hat{n})$. These are

$$
A=L(X, \hat{\mathbf{n}}) \equiv \cosh \frac{\chi}{2} \sigma_{0}-\hat{\mathbf{n}} \cdot \vec{\sigma} \sinh \frac{X}{2}=e^{\hat{\mathbf{n}} \cdot \vec{\sigma}(X / 2)}
$$

and $A=-L(X, \hat{n})$.

Thus, the group $\operatorname{SL}(2, C)$ (Special Linear group in two complex dimensions) maps $2: 1$ onto the group $S O(3,1)$. 


\subsection{Higher Dimensional Repesentations of SL(2,C)}

The discussion of higher dimensional representations of $\mathrm{SL}(2, \mathrm{C})$ is exactly the same as the discussion of higher dimensional representations of SU(2) (\$1.4 through Eq. 1-24), with $\vec{x}$ replaced by $x$, r replace by $\ell$, and $R$ replaced by $L$. The net result is that $D^{S}(L)$ is given by Eq. 1-24 $\left(R_{i j}+L_{i j}\right)$.

The matrices, $D^{5}(L)$, obtained from this procedure are a representation of $S L(2, C)$ and they form an irreducible set. However, they are not unitary and there is one (versus none in SU(2)) unitarily inequivalent $2 s+1$ dimensional representation. (D' is unitarily equivalent to $D$ if there exists a unitary matrix $U$ such that $D^{\prime}=\cup D U^{\dagger}$.) The two unitarily inequivalent representations of $S L(2, C)$ can be taken to be $D^{S}(L)$ and $D^{S}\left(I^{*}\right) \cdot 45$ 
Chapter 2

REIATIVISTIC SINGLE-PARTICLE STATES WITH SPIN

In quantum mechanics, it is postulated that the possible states of any physical system can be associated with vectors, called "state vectors", in a Hilbert space, $\mathcal{H}^{7}$ a Hilbert space is a complete linear vector space with a scalar product. A vector in $Z$ is denoted by $\psi$ and the scalar product is denoted by $\left(\psi_{1}, \psi_{2}\right)$. The scalar product is a complex number and has the property $\left(\psi_{1}, \psi_{2}\right)=$ $\left(\psi_{2}, \psi_{1}\right)^{*}$. Further, if $\alpha$ and $\beta$ are complex numbers, $\left(\psi_{3}, \alpha \psi_{1}+\beta \psi_{2}\right)=$ $\alpha\left(\psi_{3}, \psi_{1}\right)+\beta\left(\psi_{3}, \psi_{2}\right)$ and $\left(\alpha \psi_{1}+\beta \psi_{2}, \psi_{3}\right)=\alpha^{\star}\left(\psi_{1}, \psi_{3}\right)+\beta^{*}\left(\psi_{2}, \psi_{3}\right)$. The norm of $\psi$ is defined by $|\psi|=(\psi, \psi)^{\frac{3}{2}}$. In the Dirac notation, vectors are denoted by $|\psi\rangle$, the scalar product is $\left\langle\psi_{1} \mid \psi_{2}\right\rangle=\left\langle\psi_{2} \mid \psi_{1}\right\rangle^{*}$, and $\left\langle\psi_{3} \mid \alpha \psi_{1}+\beta \psi_{2}\right\rangle=\alpha\left\langle\psi_{3} \mid \psi_{1}\right\rangle+\beta\left\langle\psi_{3} \mid \psi_{2}\right\rangle$.

There is considerable freedom in choosing the association between the physical states of a system and the corresponding state 
vectors in $\mathcal{H}$. It can be shown, 7,45 however, that all physical phenomena in quantum mechanics can be described by restricting the state vectors to unit rays. A unit ray is the set of all vectors that have unit norm and are related by a phase.

Transformations from one physical state to another are described by operators that act within $\mathcal{A}$. Wigner's theorem ${ }^{39}$ says that these operators can be chosen to be either unitary operators or anti-unitary operators. A unitary operator, $U$, has the properties: $\mathrm{U}^{\dagger}=\mathrm{U}^{\dagger} \mathrm{U}=1$ and $U(\alpha|\psi\rangle)=\alpha U|\psi\rangle$, where $\alpha$ is a complex number. An anti-unitary operator, $A$, has the properties: $A A^{\dagger}=A^{\dagger} A=1$ and $A(\alpha|\psi\rangle)=a^{*} A|\psi\rangle$. Transformations that are continuously connected to the identity (e.8., translations and Lorentz transformations) can be associated with unitary operators. 45 Improper transformations (e.g., space inversion and time reversal ( $\$ 1.5)$ ) must be handled on a case by case basis. These transformations will be discussed in $\$ 2.3$.

A complete, orthonormal $\left(\left\langle\psi_{i} \mid \psi_{j}\right\rangle=\delta_{i j}\right)$ basis set of state vectors for $\not$ can be constructed by considering a maximum set of commuting transformations of the system being described. That is, a largest subset of all transformations, $T$, that have the property $T_{i} T_{j}=T_{j} T_{i}$. These transformations are associated with operators in $\Psi\left(\mathrm{T}_{i}+0_{i}\right)$, and the state vectors are labeled by the operator eigenvalues. Examples of this procedure will be given below. 
Poincaré transformations are one set of transformations that can be applied to a physical system. A Poincaré transformation consists of a translation by an amount $a \equiv\left(a_{0}, \vec{a}\right)$ and a Lorentz transformation $\ell(\ell \in \operatorname{SO}(3,1))$.

$$
P(a, 2): x \rightarrow x^{\prime}=a+2 x
$$

However, because of the $2: 1$ homomorphism between $S L(2, C)$ and So $(3,1)$, it is more useful to consider the effect of a Poincaré transformation on the $2 \times 2$ matrix $x \cdot 0$. In this case ( $\$ 1.6)$,

$$
\theta(a, L): x \cdot \sigma \rightarrow x^{\prime} \cdot \sigma=a \cdot \sigma+L x \cdot \sigma L^{\dagger}
$$

where $\mathrm{L}$ is an element of $\operatorname{SL}(2, C)$. A sequence of two Poincaré transformations is given by

$$
\begin{aligned}
\theta\left(a_{2}, L_{2}\right) P\left(a_{1}, L_{1}\right): x \cdot \sigma \rightarrow x^{\prime} \cdot \sigma= & a_{2} \cdot \bar{\sigma}+I_{2} a_{1} \cdot \sigma L_{2}^{\dagger} \\
& +\left(L_{2} L_{1}\right) x \cdot \sigma\left(L_{2} L_{1}\right)^{\dagger}
\end{aligned}
$$

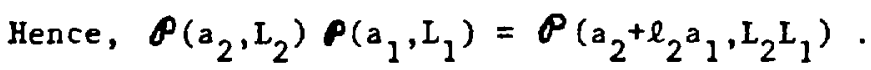

The Poincaré transformations are continuously connected to the identity and can be represented by unitary operators, $U(\theta)$. However, because physical states can at best be associated with unit rays, all representations of the Poincaré group are ray representations. That is 


$$
U\left(\theta_{2}\right) U\left(\theta_{1}\right)=e^{i \phi} U\left(\theta_{2} \phi_{1}\right)
$$

where the phase $\phi$ depends on the particular choice of the $\theta$ representation. If $\mathcal{P}$ is paramaterized by elements of $\operatorname{SL}(2, C)$, it can be shown ${ }^{45}$ that the phase can be set to zero.

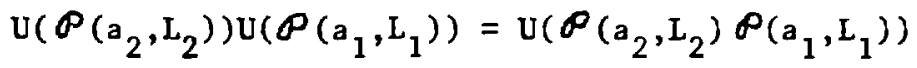

If $P$ is paramaterized by elements of $S O(3,1)$, the phase can only be specified up to a sign.

$$
U\left(P\left(a_{2}, l_{2}\right)\right) U\left(P\left(a_{1}, l_{1}\right)\right)= \pm U\left(P\left(a_{2}, l_{2}\right) P\left(a_{1}, l_{1}\right)\right)
$$

The sign in Eq. 2-6 is left undetermined because of the 2:1 homomorphism between $\operatorname{SL}(2, C)$ and $\operatorname{SO}(3,1)(\$ 1.5)$. This is why one must deal exclusively with the covering groups, $\operatorname{SI}(2, \mathrm{C})$ and its subgroup $\mathrm{SU}(2)$, in quantum mechanics.

\subsection{The Definition of Single-Particle State Vectors}

Consider the system consisting of a single particle. Within the Poincaré group, translations commute with one another. Hence, the single-particle state can be labeled by eigenvalues of the translation operator, $U(P(a, 1))$, 


$$
v(P(a, 1))|p \alpha\rangle \equiv e^{i p \cdot a}|p \alpha\rangle
$$

The four-vector $\mathrm{p}\left(\mathrm{p} \equiv\left(\mathrm{p}_{0}, \overrightarrow{\mathrm{p}}\right)\right)$ is defined to be the four-momentum of the particle and $\alpha$ denotes other state vector labels. The unitarity of the operator $U(P(a, 1))$ has been used to write its eigenvalues in the form $e^{i p \cdot a}\left(p \cdot a \equiv p_{0} a_{0}-\vec{p} \cdot \vec{a}\right)$.

A single particle state of mass $m$ is defined to be a state whose four-momentum $p$ satisfies the conditions $m=(p \cdot p)^{\frac{3}{2}}, p \cdot p>0$, and $P_{0}>0$. The single-particle Hilbert space is restricted to just those state vectors that satisfy these conditions. This restriction is not violated by proper Lorentz transformations (i.e., $\ell_{p}$ is also an element of the restricted set of four-momenta) and will be used in $\$ 2.3$ to define the space-inversion and time-reversal operators. In chis dissertation, the completeness condition for the restricted single-particle Hilbert space is specified by the following covariant phase-space integral:

$$
\begin{aligned}
1 & =\sum_{\alpha} \int d^{4} p \delta^{+}\left(p^{2}-m^{2}\right) \mid p \alpha>\langle p \alpha| \\
& =\sum_{\alpha} \int \frac{d^{3} p}{2 \varepsilon(|\vec{p}|)} \mid p \alpha>\langle p \alpha|
\end{aligned}
$$

where $\delta^{+}\left(p^{2}-m^{2}\right)=\theta\left(p_{0}\right) \delta\left(p^{2}-m^{2}\right)(\theta(x)=0$ if $x<0$ and 1 if $x>0)$ and $\varepsilon(|\vec{p}|)=\left(\vec{p}^{2}+m^{2}\right)^{\frac{1}{2}}$. This completeness condition.implies that the normalization of the basis state vectors is invariant and given by 


$$
\left\langle p^{\prime} \alpha^{\prime} \mid p \alpha\right\rangle=2 \varepsilon(|\vec{p}|) \delta^{3}\left(\overrightarrow{p^{\prime}}-\vec{p}\right) \delta_{\alpha^{\prime} \alpha}
$$

An invariant normalization is necessary for a unitary operator to yield a unitary representation of the Lorentz group (e.g., Eq. 2-11).

Additional labels for the single-particle state vector can be determined by considering the state vector $\{U(P(O, L)) \mid p a>\}$. Under a translation (cf., Egs. 2-3 and 2-4), this state vector is transformed as follows:

$$
\begin{aligned}
& U(P(a, 1))\{U(P(0, I)) \mid P \alpha>\} \\
& =U(\sigma(a, 1) \beta(0, L))|p \alpha\rangle \\
& =U\left(\theta(0,1) \rho\left(l^{-1} a, 1\right)\right)|p \alpha\rangle \\
& =e^{i p \cdot l^{-1} a}\{U(P(0, L)) \mid p a>\} \\
& =e^{i \ell_{p} \cdot a}\{U(\mathscr{O}(0, L))|p \alpha\rangle\}
\end{aligned}
$$

Hence, the state vector $\{U(P(0, L)) \mid p \alpha>\}$ has the four-momentum $p^{\prime} \equiv \ell p$. This implies that

$$
U(P(0, L))|p \alpha\rangle=N(\ell ; p) \sum_{\beta} M_{\beta \alpha}(L ; p)\left|\ell_{p} \beta\right\rangle
$$

where $M(L ; p)$ is a unitary matrix $\left(M^{\dagger} M=M^{\dagger}=1\right)$ and $N(\ell ; p)$ is a normalization factor. $N(l ; p)=1$ if an invariant normalization of the basis state vectors is used (Eq. 2-9). 
Eq. 2-11 allows one to define the state vector $|p \alpha\rangle$ as follows :

i) Pick one state vector, $|\dot{p} \alpha\rangle$, and a single set of Lorentz transformations, $c(p, \stackrel{0}{p})$; such that, $p=c(p, \stackrel{0}{p}) \dot{p}$.

ii) Define the state vector $|p| \alpha\rangle$ by the relation

$$
|p \alpha\rangle \equiv U(P(0, C(p, \stackrel{\circ}{p}))|\stackrel{\circ}{p} \alpha\rangle
$$

The state vector $|\dot{p} \alpha\rangle$ is called the "fundamental state vector", is calle: the "fundamental four-momentum", and the set $c(p, \stackrel{0}{p})$ is called the "complementay set" of Lorentz transformations. This definition is made possible by the following result:

Eq. 2-11 says that there must exist a basis set of state vectors with the property

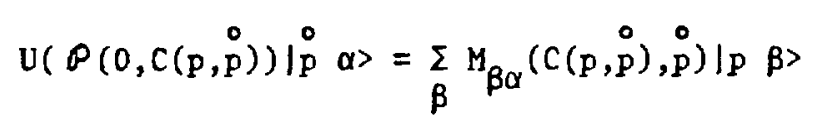

where $M$ is unitary and $M(C(\dot{p}, \stackrel{0}{p}) ; p)=M(1 ; p)=1$. Hence, one can always define a new basis

$$
\left.\left|p \alpha>1 \equiv \sum_{\beta} M_{\beta \alpha}(C(p, \stackrel{p}{p}) ; \stackrel{0}{p})\right| p \beta\right\rangle
$$


that satisfies $U(P(0, C(p, \dot{p})|\dot{p} \alpha\rangle=|p \alpha\rangle$, and agrees with the definition of $|p \alpha\rangle$ in Eq. 2-12.

Different choices of the complemestary set, $c(p, \dot{p})$, will yield different sets of basis state vectors for the single-particle Hilbert space. Two of the most commonly used complementary sets will be defined in $\$ \S 2.1 .2$ and 2.1 .3 .

For assive particle, the fundamental four-momentum $\dot{p}$, can be set equal to the particle's rest frame four-momentum.

$$
\dot{p}=\left(\begin{array}{l}
m \\
0 \\
0 \\
0
\end{array}\right)
$$

where $m$ is the particle's mass. This value of the fundamental four-momentum is rotationally invariant (i.e., $\stackrel{0}{\mathrm{r}}=\dot{0}$ ), which implies

$$
U(\theta(0, R))|\dot{p} \alpha\rangle=\sum_{\beta} M_{\beta \alpha}(R ; \dot{p})|\dot{p} \beta\rangle
$$

The fundamental state vector for a particle of spin $s$ is then defined 34,35 by setting $M_{\beta \alpha}(R ; \dot{p})=D_{\beta \alpha}^{s}(R)$, where $D^{5}(R)$ is a $2 s+1$ dimensional representation of the rotation group, $\operatorname{SU}(2)$ (\$1.4). 


$$
U(R)|\dot{p} \lambda \gamma\rangle=\sum_{\lambda^{\prime}} D_{\lambda^{\prime}}^{S} \lambda^{(R)}\left|\dot{p} \lambda^{\prime} \gamma\right\rangle
$$

where $\gamma$ denotes other state vector labels. (Note: In the remainder of this dissertation, the additional state vector labels will not be explicitely written and the notation $U(L) \equiv U(\mathcal{P}(0, L))$ will be used.) The commuting transformation that corresponds to the label $\lambda$ is $R(\phi, \hat{z})$. ([R( $(\phi, \hat{z}), R(\theta, \hat{x})] \neq 0$ and $[R(\phi, \hat{z}), R(\theta, \hat{y})] \neq 0$.

The rotation group, $\mathrm{SU}(2)$, is called the "little group" that belongs to the fundamental four-momentum, $\stackrel{0}{p}$. The little group is the group of all Lorentz transformations that leave $\stackrel{\circ}{p}$ unchanged.

\section{1 .1 Basis $^{6,45}$}

The $Z$ set of basis state vectors for the single-particle Hilbert space is defined by the following choice of the complementary set of Lorentz transformations:

$$
c(p, \ddot{p}) \equiv c_{z}(p) \equiv L\left(x_{p}, \hat{p}\right)
$$

$$
\text { where } \quad \begin{aligned}
\mathbf{p} \equiv\left(\begin{array}{c}
\mathrm{p}_{0} \\
\overrightarrow{\mathrm{p}}
\end{array}\right) \quad: \mathrm{P}_{0}=\varepsilon(|\overrightarrow{\mathrm{p}}|) \equiv\left(|\overrightarrow{\mathrm{p}}|^{2}+m^{2}\right)^{\frac{1}{2}} \\
\hat{\mathbf{p}}=(\sin \theta \cos \phi, \sin \theta \sin \phi, \cos \theta) \quad: \begin{array}{l}
0 \leqq \phi \leqq 2 \pi \\
0 \leqq \theta \leqq \pi
\end{array}
\end{aligned}
$$

and

$$
\tanh x_{p}=\frac{|\vec{p}|}{p_{0}}
$$


The $Z$ basis is then defined by Eq. 2-12.

$$
|p \lambda\rangle_{z} \equiv U\left(C_{z}(p)\right)|\stackrel{0}{p} \lambda\rangle_{z}=U\left(L\left(x_{p}, \hat{p}\right)\right)|\dot{p} \lambda\rangle_{z}
$$

\subsubsection{Helicity Basis 47}

The helicity set of basis state vectors is defined by the following choice of the complementary set:

$$
C(p, \stackrel{\circ}{p}) \equiv C_{b}(p)=R(\phi, \hat{z}) R(\theta, \hat{y}) L\left(\chi_{p}, \hat{z}\right)
$$

where $p, x_{p}$, and $\hat{p}$ are defined as in Eq. 2-16. The following notation is also used:

$$
\begin{array}{ll} 
& R(\hat{p}) \equiv R(\phi, \theta) \equiv R(\phi, \hat{z}) R(\theta, \hat{y}) \\
\text { and } \quad & Z(x) \equiv L(x, \hat{z}) .
\end{array}
$$

The helicity basis is then defined by Eq. 2-12.

$$
|\mathrm{p} \lambda\rangle_{h} \equiv \mathrm{U}\left(C_{h}(p)\right)|\stackrel{0}{p} \lambda\rangle=U\left(R(\hat{p}) Z\left(x_{p}\right)\right)|\stackrel{0}{p} \lambda\rangle
$$

The relation between the $Z$ basis, $|p \lambda\rangle_{z}$, and the helicity basis, Ip $\lambda>_{h}$, is determined by applying the proof of Eq. 1-15 to the Lorentz groups. The result is 


$$
L(X, \hat{\mathbf{n}}) \equiv R^{-1} L(X, \mathrm{rn}) R
$$

where $R$ is an arbitrary rotation. This identity implies

$$
I\left(x_{p}, \hat{p}\right)=R(\hat{p}) Z\left(X_{p}\right) R(\hat{p})^{-1}
$$

Hence, $|p v\rangle_{z}=\sum_{\lambda} D_{\lambda v}^{s}\left(R(\hat{p})^{-1}\right)|p \lambda\rangle_{h}$

and $\quad|p \lambda\rangle_{h}=\sum_{v} D_{v \lambda}^{S}(R(\hat{p}))|p v\rangle_{z}$

The meaning of the term "axis of spin quantization" is demonstrated by the following relations (use Eqs. 1-15, 1-25, and $[R(\zeta, \hat{z}), Z(\chi)]=0):$

$$
U(R(\zeta, \hat{p}))|p \lambda\rangle_{h}=e^{-i \lambda \zeta}|p \lambda\rangle_{h}
$$

and $\quad U(R(\zeta, \hat{z}))|p \lambda\rangle_{z}=e^{-i \lambda \zeta}|p \lambda\rangle_{z}$

In the $z$ basis, the operator for rotations about the $\hat{z}$ axis is diagonal; hence, $|p \lambda\rangle_{z}$ is said to have its spin quantized along the $\hat{z}$ axis. In the helicity basis, the operator for rotations about the vector $\hat{p}$ is diagonal; hence, $|p \lambda\rangle_{h}$ is said to have its spin quantized along the direction of the momentum vector. 


\subsection{Lorentz Transformations of Single-Particle States}

Eqs. 2-5, 2-11, and 2-12 imply that

$$
U(L)|p \lambda\rangle=U(L C(p))|\dot{p} \lambda\rangle=\sum_{v} M_{v \lambda}(L ; p) U(C(l p))|\dot{p} v\rangle
$$

Hence, $v\left(C(\ell p)^{-1} L C(p)\right)|\dot{p} \lambda\rangle=\sum_{v} M_{v \lambda}(L ; p)|\dot{p} v\rangle$

But the only Lorentz transformations that leave $\stackrel{\circ}{p}$ unchanged are rotations; thus, $C\left(\ell_{p}\right)^{-1} L C(p)$ must be a rotation and $M_{v \lambda}(L ; p)$ is a rotation matrix. The following notation will be used:

$$
R(L ; p) \equiv C(\ell p)^{-1} L C(p) \text { and } M_{v \lambda}(L ; p)=D_{v \lambda}^{s}(R(L ; p))
$$

\subsubsection{Lorentz Transformations: Z Basis}

Applying Eq. 2-26 in the $Z$ basis (\$2.1.1) yields the following useful results:

i) If $L$ is a rotation, $L \equiv R$, then (cf., Eq. 2-21)

$$
R(R ; p)=L\left(x_{p}, r \hat{p}\right)^{-1} R I\left(x_{p}, \hat{p}\right)=R
$$

Hence, $U(R)|p \lambda\rangle_{z}=\sum_{v} D_{v \lambda}^{s}(R)|p\rangle_{z}$ 
ii) If $L$ is a boost in the $\hat{z}$ direction, $I \equiv Z(x)$, then

$$
\begin{aligned}
R(Z(x) ; p) & =L\left(x_{p^{\prime}}, \hat{p}^{\prime}\right)^{-1} Z(x) L\left(x_{p}, \hat{p}\right) \\
& =R\left(\hat{p}^{\prime}\right) Z\left(x_{p^{\prime}}\right)^{-1} R\left(\hat{p}^{\prime}\right)^{-1} Z(x) R(\hat{p}) Z\left(x_{p}\right) R(\hat{p})^{-1}
\end{aligned}
$$

where $p^{\prime} \equiv z(x) p$. This equation implies that the quantity $Z\left(x_{p^{\prime}}\right)^{-1} R\left(\hat{p}^{\prime}\right)^{-1} Z(X) R(\hat{p}) Z\left(X_{p}\right)$ must also be a rotation, call it $R_{h^{\prime}}$. Further, since $z(x)$ does not change the $\hat{x}$ or $\hat{y}$ components of $p$, $\phi^{\prime}$ must equal $\phi$, and $R_{h}$ is at most a rotation about the $\hat{y}$ axis.

$$
R_{h} \equiv R(\xi, \hat{y})=Z\left(x_{p},\right)^{-1} R\left(\theta^{*}, \hat{y}\right)^{-1} Z(x) R(\theta, \hat{y}) Z\left(x_{p}\right)
$$

Hence, $U(z(x))|p \lambda\rangle_{z}=\sum_{v} e^{-i(v-\lambda) \phi} d_{v \lambda}\left(\theta^{\prime}+\zeta-\theta\right)|z(x) p \quad v\rangle$

The value of the "Wigner rotation angle" $\zeta$ is easily determined by considering the boost $2(x)$ as a rotation about the $\hat{z}$ axis. That is, let

$$
\begin{aligned}
Z(x) & \equiv \sigma_{0} \cosh \frac{x}{2}-\sigma_{3} \sinh \frac{x}{2} \\
& =\sigma_{0} \cos \left(-\frac{i x}{2}\right)-i \sigma_{3} \sin \left(-\frac{i x}{2}\right) \\
& =R(-i x, \hat{z})
\end{aligned}
$$

This result and Eq. 2-30 then imply that the rotation $R(\zeta, \hat{y})$ can be obtained from the "spherical triangle" that is shown in Fig. 2.1. 
(Consider the line that is traced by the $\hat{y}$ axis on a sphere when the sphere undergoes the "rotations" shown in Eg. 2-30. Also, see $\S 2.2 .2)$

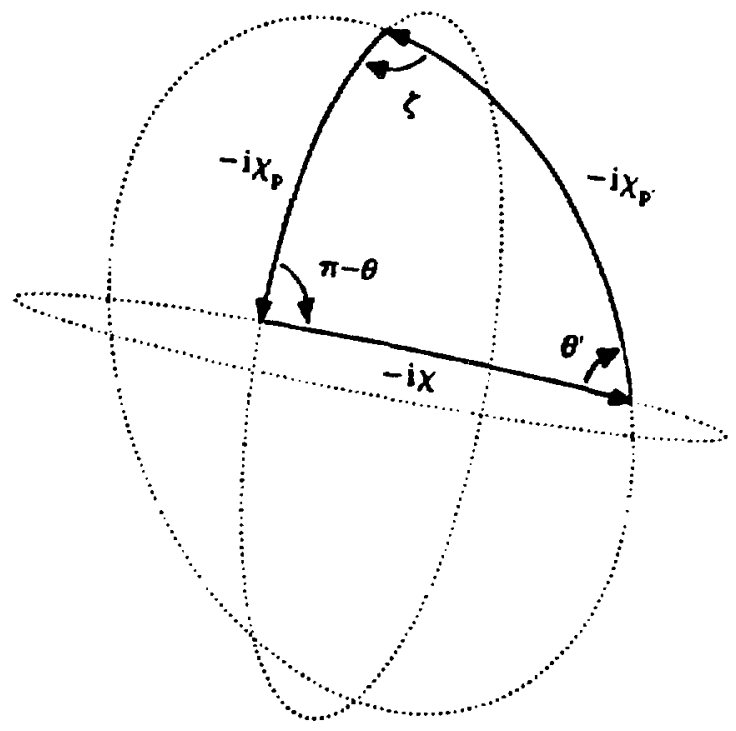

Fig. 2.1 Rotation triangle for the state vector $Z(x) \mid p \lambda>$.

$$
\begin{aligned}
& \text { Hence, } \quad \frac{\sin \zeta}{\sinh \chi}=\frac{\sin \theta^{\prime}}{\sinh \chi_{p}}=\frac{\sin \theta}{\sinh \chi_{p^{\prime}}} \\
& \cosh \chi_{p}=\cosh \chi \cosh \chi_{p^{\prime}}-\sinh \chi \sinh \chi_{p^{\prime}} \cos \theta^{\prime} \\
& \cosh \chi=\cosh \chi_{p} \cosh \chi_{p^{\prime}}-\sinh \chi_{p} \sinh \chi_{p} \cdot \cos \xi
\end{aligned}
$$

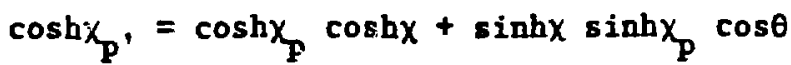


and

$$
\tan \zeta=\frac{\sinh \chi \sin \theta}{\sinh \chi_{p} \cosh \chi+\cosh \chi_{p} \sinh \chi \cos \theta}
$$

If the velocity of the Lorentz boost is $u=\tanh x$, then this equacan also be written as

$$
\tan \zeta=\frac{u \sin \theta}{\frac{|\vec{p}|}{m}+u \mathbb{R}_{0} \cos \theta}
$$

(Note: This method was developed by Wick in the appendix to Ref. 48.)

These results also show that the angle $\zeta$ is simply the angle between the velocity vector $\vec{u}_{p}=\vec{p} / m$ and the velocity vector $\vec{u}_{p^{\prime}}=\vec{p}^{\prime} / m$. In the nonrelativistic limit, $|\vec{p}| \ll m$, this angle is $\theta-\theta^{\prime}$; so, in this limit,

$$
v(z(x))|p v\rangle_{z} \stackrel{\text { n.r. }}{\longrightarrow}|z(x) p \quad v\rangle_{z}
$$

and there is no spin precession.

\subsubsection{Lorentz Transformations: Helicity Basis}

In the helicity basis, Eq, 2-23 can be applied to obtain the following results: 


$$
U(R)|p \lambda\rangle_{h}=\sum_{v} D_{v \lambda}^{s}\left(R(r \hat{p})^{-1} R R(\hat{p})\right)|p v\rangle_{h}
$$

This implies that $R(R ; p)=R(r \hat{p})^{-1} R R(\hat{p})$. But, $R(R ; p)$ also equals $Z\left(x_{p}\right) R(r \hat{p}){ }^{1} R R(\hat{p}) Z\left(x_{p}\right)$. Hence, $\left[Z\left(x_{p}\right), R(R ; p)\right]=0$; so that, $R(R ; p)$ must be a rotation about the $\hat{z}$ axis.

$$
\begin{aligned}
& R(R ; p) \equiv R(\xi, \hat{z})=R(r \hat{p})^{-1} R R(\hat{p}) \\
& \text { And } \\
& U(R)|p \lambda\rangle_{h}=e^{-i \lambda \zeta}|p \lambda\rangle_{h}
\end{aligned}
$$

The following are specific examples of this result:

a) If $R$ is a rotation about the $\hat{z}$ axis, $R \equiv R(\xi, \hat{z})$, then

$$
\zeta=0 \text { if } 0 \leqq \phi+\xi<2 \pi
$$

and

$$
\zeta=2 \pi \text { if } 2 \pi \leqq \phi+\xi<4 \pi \text {. }
$$

where $\hat{\mathbf{p}}$ has the azimuthal angle $\phi$. A crucial point here is that the domain of definition of the complementary set requires that the azimuthal angles of $\hat{\mathbf{p}}^{\prime} \equiv \mathbf{r}(\xi, \hat{z}) \hat{\mathbf{p}}$ lie in the range $0 \leqq \phi^{\prime}<2 \pi$. This is important because $\zeta=2 \pi$ is not equivalent to $\zeta=0$. This fact has not always been noted in the literature. 47,48

b) If $R$ is a rotation about the $\hat{y}$ axis, $R \equiv R(\xi, \hat{y})$, then $\zeta$ can be determined from the spherical triangle depicted in Fig. 2.2. (Consider the line that is traced by the $\hat{z}$ axis on a sphere when that sphere undergoes the rotations shown in Eq. 2-39 with $R=$ $R(\xi, y), R(p)=R(\phi, z) R(\theta, \hat{y})$, and $\left.r \hat{p} \equiv r(\xi, \hat{y}) \hat{p}=\hat{p}^{\prime}.\right)$ 


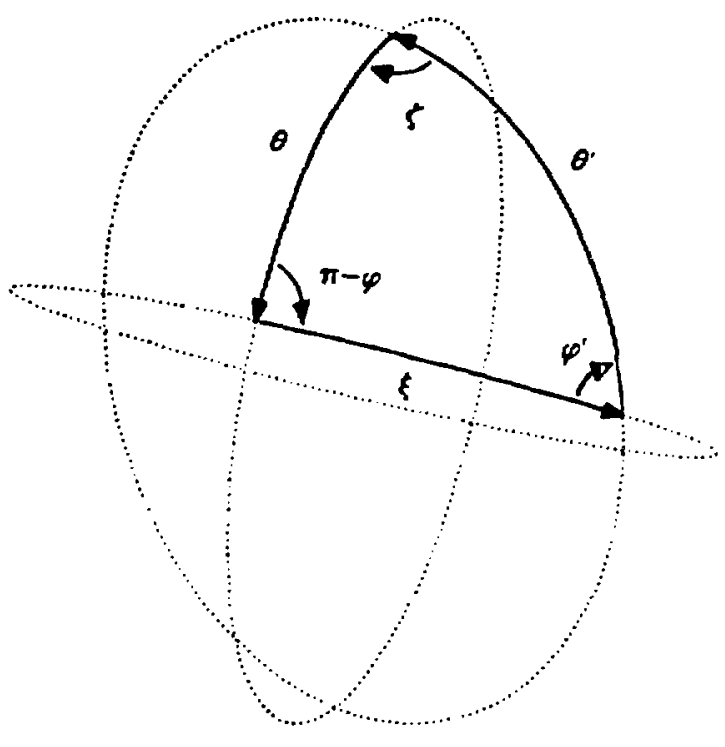

Fig. 2.2 Rotation triangle for the state vector $R(\xi, \hat{y})|p \quad \lambda\rangle_{h}$.

In this figure, $\mathrm{p}^{\prime} \equiv \mathbf{r}(\xi, \hat{\mathrm{y}}) \mathrm{p}$ and

$$
\begin{aligned}
& \cos \zeta=\frac{\cos \xi \sin \theta+\sin \xi \cos \theta \cos \phi}{\sin \theta^{\prime}} \\
& \sin \zeta=\frac{\sin \xi \sin \phi}{\sin \theta^{\prime}}
\end{aligned}
$$

(Note: The angle $\zeta$ can have values in the range $0 \leqq \zeta<2 \pi$ or $2 \pi \leqq \zeta<4 \pi$. The precise value of $\zeta$ can be determined by a careful consideration of the rotations that were used to obtain Fig.2.2.) 
ii) If $L$ is a boost in the $\hat{z}$ direction, $L \equiv Z(x)$, then

$$
U(z(x))|p \lambda\rangle_{h}=\sum_{v} D_{v \lambda}^{s}\left(R_{h}\right)|p \quad v\rangle_{h}=\sum_{v} d_{v \lambda}^{s}(\zeta)|p \quad v\rangle_{h}
$$

where $R_{h}$ is defined in Eq. $2-30$ and $\zeta$ is given by $E q \cdot 2-35$.

\subsection{Improper Transformations}

Until now, only Lorentz transformations with det $\ell=1$ and $\ell_{00} \geqq 1$ have been discussed. These transformations constitute the proper (or orthochronous) Lorentz group, $S O(3,1)$. In order to complete the analysis of all possible Lorentz transformations (Table 1.1), one must also consider the improper operations of space-inversion, $d_{s}$, and time-reversal, $d_{t}$.

The first step in the evaluation of an improper transformation is to associate with that transformation an operator in the Hilbert space of single-particle state vectors. Further, because these transformations are not continuously connected to the identity, one must determine in each case whether that operator is unitary or anti-unitary. The effect of the transformation on the single-particle state vectors can then be evaluated, and the need for additional state vector labels can be determined. 


\subsubsection{Space Inversion (Parity)}

The space-inversion transformation, $\mathcal{L}_{s}$, is defined by

$$
\mathcal{L}_{s}: x \rightarrow x^{\prime}=\left(\begin{array}{c}
x_{0} \\
-\vec{x}
\end{array}\right) \equiv I_{s} x
$$

where $\left(I_{s}\right)_{00}=1,\left(I_{s}\right)_{i i}=-1$, and $\left(I_{s}\right)_{\mu \nu}=0$ if $\mu \neq \nu$. In terms of the $2 \times 2$ matrix $x \cdot \sigma$, this transformation is given by

$$
d_{s}: x \cdot \sigma \rightarrow x^{\prime} \cdot \sigma=x_{0} \sigma_{0}+\vec{x} \cdot \vec{\sigma}=I_{s} x \cdot \sigma=\zeta(x \cdot \sigma)^{*} \zeta^{\dagger}=\zeta^{\dagger}\left(x \text { j; } j^{2} \zeta \quad(2-45)\right.
$$

where $\zeta=\left(\begin{array}{cc}0 & -1 \\ 1 & 0\end{array}\right)$

The following results were used to obtain Eq. 2-6:5:

i) $\quad \sigma_{1}^{*}=\sigma_{1}, \quad \sigma_{2}^{*}=-\sigma_{2}, \sigma_{3}^{*}=\sigma_{3}$

ii) $\zeta \sigma_{1} \zeta^{\dagger}=-\sigma_{1}, \zeta \sigma_{2} \zeta^{\dagger}=\sigma_{2}, \zeta \sigma_{3} \zeta^{\dagger}=-\sigma_{3}$

Also note that $\zeta=-i \sigma_{2}=R(\pi, \hat{y}), \quad \zeta^{-1}=\zeta^{T}=\zeta^{\dagger}$,

and, if $M$ is any $2 \times 2$ unimodular matrix, $M^{-1}=\zeta M^{T} \zeta^{\dagger}$.

An operator, $U\left(\mathcal{d}_{5}\right)$, can now be associated with the spaceinversion tiausformation. Two questions immediately arise:

i) Recalling that the operator representations are ray represeatations (Eg. 2-4), how does one evaluate the sequence of operations $v\left(d_{s}\right) U(\theta)$ ? 
ii) Is the operator $\mathrm{U}\left(\mathscr{f}_{s}\right)$ unitary or anti-unitary? Question (i) is answered by considering the transformation

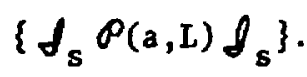

$$
\left\{\mathcal{J}_{s} P(a, L) \mathcal{L}_{s}\right\}: x \cdot \sigma \rightarrow x^{\prime} \cdot \sigma=I_{s} a \cdot \sigma+\left(\zeta L^{*} \zeta^{\dagger}\right) x \cdot \sigma\left(\zeta L^{*} \zeta^{\dagger}\right)^{\dagger}
$$

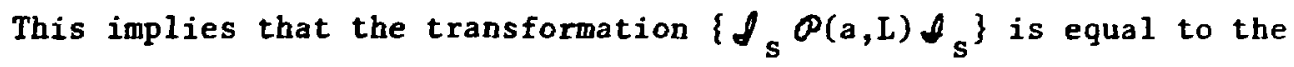
transformation $\boldsymbol{\rho}\left(I_{s} a, \zeta I^{*} \zeta^{\dagger}\right)$ provided $\left(\zeta I^{\frac{\hbar}{*}} \zeta^{\dagger}\right)$ is an element of the proper Lorentz group (i.e., $\operatorname{det} \zeta L^{*} \zeta^{\dagger}=1$ and $\operatorname{tr} \zeta L^{*} \zeta^{\dagger} \geqq 1$ ). This is easily shown to be true using the methods of Appendix B. It can then be shown 34,45 that an operator $U\left(f_{S}\right)$ that satisfies

$$
\mathrm{U}\left(\ell_{\mathrm{s}}\right) \mathrm{U}(\theta)=\mathrm{U}\left(\phi_{\mathrm{s}} \rho \phi_{\mathrm{s}}\right) \mathrm{U}\left(\ell_{\mathrm{s}}\right)
$$

does exist.

Question (ii) is answered by considering a translation of the state vector $\left\{U\left(d_{s}\right) \mid p \lambda>\right\}$.

$$
\begin{aligned}
& \mathrm{U}(\theta(\mathrm{a}, 1))\left\{\mathrm{U}\left(\delta_{\mathrm{s}}\right)|\mathrm{p} \lambda\rangle\right\}=\mathrm{U}\left(\delta_{\mathrm{s}}\right) \mathrm{U}\left(\delta_{\mathrm{s}} \sigma(\mathrm{a}, 1) \phi_{\mathrm{s}}\right)|\mathrm{p} \lambda\rangle \\
& =u\left(\theta_{s}\right) U\left(\theta\left(I_{s} a, 1\right)\right) \mid p \lambda \\
& =U\left(\delta_{s}\right) e^{i p \cdot I_{s} a}|p \lambda\rangle \\
& =U\left(\theta_{s}\right) e^{i I_{s} p \cdot a}|p \lambda\rangle
\end{aligned}
$$


Hence, if $U\left(d_{\delta}\right)$ is unitary then $\left\{U\left(d_{s}\right) \mid p \lambda>\right\}$ is state vector with four-momentum $I_{s} p$; whereas, if $U\left(d_{s}\right)$ is anti-unitary - it is a state vector with four-momentum $-I_{s} p$. Requiring $P_{0}$ to be positive (i.e., requiring $\left\{U\left(\mathscr{f}_{\mathrm{s}}\right) \mid \mathrm{p} \lambda>\right\}$ to be a state vector in the restricted single-particle Hilbert space) then implies that $\mathrm{U}\left(\delta_{S}\right)$ is a unitary operator.

The spin properties of $\left\{U\left(\mathcal{L}_{s}\right) \mid p \quad \lambda>\right\}$ are determined by considering a rotation of the state vector $\left\{U\left(d_{s}\right) \mid p \lambda \lambda\right\}$.

$$
v(R)\left\{U\left(\delta_{s}\right) \mid \dot{p} \lambda>\right\}=v\left(d_{s}\right) U\left(\zeta R^{*} \zeta^{\dagger}\right)|\dot{p} \lambda\rangle=\sum D_{v \lambda}^{s}(R\}\left\{U\left(d_{s}\right) \mid \dot{p} \lambda>\right\}
$$

where Eqs. 1-12, 2-47, and 2-48 have been used to show that $\zeta R^{*} \zeta^{\dagger}=R . \quad \bar{E} q .2-54$ implies that $\left\{U\left(\mathcal{L}_{S}\right) \mid \dot{p} \lambda>\right\}$ transforms under a rotation as a state vector of spin s and spin projection $\lambda$.

Hence, $\left.\left.\left.U\left(\mathcal{d}_{\mathrm{s}}\right)\right|_{\mathrm{p}} ^{0} \lambda \gamma\right\rangle=\sum_{\gamma^{\prime}} \mathrm{M}^{\prime} \gamma^{\prime} \gamma^{\mid 0} \lambda \gamma^{\prime}\right\rangle$

where the additional state vector labels shown in Eq. 2-14 have been explicitely written and $M$ is a unitary matrix. Further application of $u\left(d_{s}\right)$ yields

$$
\left.u\left(d_{s}\right)^{2}|\dot{p} \lambda \gamma\rangle=\sum_{\gamma^{\prime}}\left(\mathbb{M}^{2}\right) y^{\prime} \gamma^{\mid 0} \lambda \gamma^{\prime}\right\rangle=|\dot{p} \lambda \gamma\rangle
$$

where the phase in $U\left(d_{s}\right)^{2}=e^{i \phi} U\left(d_{s}^{2}\right)=e^{i \phi}$ has been chosen to be 
zero. Thus, $M^{2}=1$, one can take $M_{\gamma^{\prime}} \gamma=\eta_{p}{ }^{\delta} \gamma^{\prime} \gamma$ with $\eta_{p}= \pm 1$, and the fundamental state vector for a particle of parity $\eta_{p}\left(\eta_{p}= \pm 1\right)$ can be defined by

$$
u\left(t_{s}\right)\left|\stackrel{p}{p} \lambda \eta_{p}\right\rangle=\eta_{p}\left|\stackrel{0}{p} \lambda \eta_{p}\right\rangle
$$

(Note: In the remainder of this dissertation, the parity label will not be explicitely written.)

Having defined the action of the space-inversion transformation on the fundamental state vector, its action on an arbitrary basis state vector is determined as follows:

$$
\begin{aligned}
& \left.U\left(\delta_{s}\right)|p \lambda\rangle=\left.U\left(d_{s}\right) U(C(p))\right|_{p} ^{0} \lambda\right\rangle=\eta_{p} U\left(\zeta C(p)^{*} \zeta^{\dagger}\right)|\dot{p} \lambda\rangle \\
& \equiv \eta_{p} \sum D_{v \lambda}^{s}\left(R_{s}(C(p))\right)\left|I_{s} p v\right\rangle
\end{aligned}
$$

where $R_{s}(C(p))$ is the following rotation:

$$
R_{s}(C(p))=C\left(I_{s} p\right)^{-1} \zeta C(p)^{*} \zeta^{\dagger}
$$

In the $Z$ basis, $\zeta C_{z}(p)^{*} \zeta^{\dagger}=L\left(\chi_{p}, I_{s} \hat{p}\right)$; so that,

$$
U\left(\mathcal{L}_{\mathrm{s}}\right)|\mathrm{p} \lambda\rangle_{\mathrm{z}}=\eta_{\mathrm{p}}\left|\mathrm{I}_{\mathrm{s}} \mathrm{p} \lambda\right\rangle_{\mathrm{z}}
$$

In the helicity basis, Eqs. 1-12, 1-41, 2-47, 2-48, and 2-49 can be used to shor that $\zeta C_{h}(p)^{\star} \zeta^{\dagger}=\zeta R(\hat{p})^{\star} z\left(\chi_{p}\right)^{\star} \zeta^{\dagger}=R(\hat{p}) \zeta Z\left(\chi_{p}\right) \xi^{\dagger}=$ $R(\hat{\mathrm{p}}) \mathrm{R}(\pi, \hat{\mathrm{y}}) Z\left(\chi_{\mathrm{p}}\right) \mathrm{R}(\pi, \hat{\mathrm{y}})^{-1}$; hence, 


$$
\begin{aligned}
U\left(\delta_{s}\right)|p \lambda\rangle_{h} & =\eta_{p} U\left(R(\hat{p}) R(\pi, \hat{y}) Z\left(x_{p}\right) R(\pi, \hat{y})^{-1}\right)|\dot{p} \lambda\rangle \\
& =\eta_{p}(-1)^{s+\lambda} U\left(R(\hat{p}) R(\pi, \hat{y}) Z\left(x_{p}\right)\right)|\dot{p}-\lambda\rangle
\end{aligned}
$$

\subsubsection{Time Reversal}

The time-revesal transformation, $d_{t}$, is defined by

$$
d_{t}: x \rightarrow x^{\prime}=\left(\begin{array}{c}
-x_{0} \\
\vec{x}
\end{array}\right) \equiv I_{t} x
$$

where $\left(I_{t}\right)_{00}=-1,\left(I_{t}\right)_{i i}=1$, and $\left(I_{t}\right)_{\mu \nu}=0$ if $\mu \neq v$. In terms of the $2 \times 2$ matrix $x \cdot \sigma$, this transformation is

$$
d_{t}: x \cdot \sigma \rightarrow x^{\prime} \cdot \sigma=-x_{0} \sigma_{0}-\vec{x} \cdot \vec{\sigma}=I_{t} x \cdot \sigma=-\zeta(x \cdot \sigma)^{*} \zeta^{\dagger}
$$

so that $\left\{\mathcal{J}_{t} P(a, L) d_{t}\right\}=P\left(I_{t} a, \zeta L^{*} \zeta^{\dagger}\right)$.

An operator, $U\left(J_{t}\right)$, can then be associated with the timereversal transformation and the procedure that was used for the space-inversion transformation can be applied to show that this operator must be anti-unitary. Furthermore, under a rotation, 


$$
\begin{aligned}
& \mathrm{u}(R)\left\{\mathrm{u}\left(\mathcal{L}_{\mathrm{t}}\right)|\dot{\mathrm{p}} \lambda\rangle\right\}=\mathrm{u}\left(d_{\mathrm{t}}\right) \mathrm{U}\left(\xi \mathrm{R}^{*} \xi^{\dagger}\right)|\dot{\mathrm{p}} \lambda\rangle \\
& =\sum_{v} D_{v \lambda}^{s}(R)^{*}\left\{U\left(\mathcal{J}_{t}\right)|\dot{p} v\rangle\right\}
\end{aligned}
$$

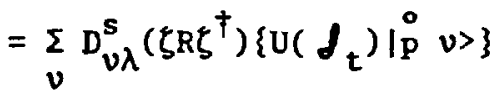

Hence, $\left\{\sum_{v} D_{v \lambda}^{s}\left(\zeta^{\dagger}\right) U\left(\mathcal{d}_{t}\right) \mid \dot{p} v>\right\}$ rotates as a state vector of spin $s$ and spin projection $\lambda$.

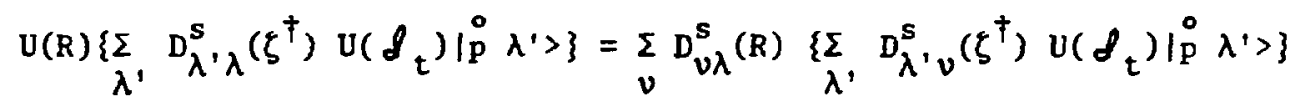

This implies that

$$
\left.u\left(d_{t}\right) \stackrel{0}{\mid p} \lambda\right\rangle=\eta_{t} \sum_{\lambda^{\prime}} D_{\lambda^{\prime} \lambda}^{s}(\zeta)\left|p \lambda^{\prime}\right\rangle=\eta_{t}(-1)^{s-\lambda}|\dot{p}-\lambda\rangle
$$

where $\eta_{t}$ is an arbitrary phase factor, which can be set equal to one. 15,45 Thus,

$$
\mathrm{U}\left(\delta_{\mathrm{t}}\right)|\stackrel{0}{\mathrm{p}} \lambda\rangle=\sum_{v} \mathrm{D}_{v \lambda}^{\mathrm{s}}(\zeta)|\stackrel{0}{\mathrm{p}} v\rangle=(-1)^{\mathrm{s}-\lambda}|\dot{\mathrm{p}}-\lambda\rangle
$$

Having defined the action of the time-reversal transformation on the fundamental state vector, its action on an arbitrary 
basis state vector is determined os follows:

$$
\begin{aligned}
u\left(d_{t}\right)|p \lambda\rangle & \left.=\left.v\left(f_{t}\right) U(C(p))\right|_{p} ^{0} \lambda\right\rangle \\
& =\sum_{v} D_{v \lambda}^{s}(\zeta) v\left(\zeta C(p)^{\star} \zeta^{\dagger}\right)|p v\rangle \\
& =\sum_{v} D_{v \lambda}^{s}\left(R_{s}(C(p)) \zeta\right)\left|I_{s} p v\right\rangle
\end{aligned}
$$

where $R_{s}(C(p))$ is given in Eq. 2-59. This implies that

$$
U\left(d_{t}\right)|p \lambda\rangle_{z}=(-1)^{s-\lambda}\left|I_{s} p-\lambda\right\rangle_{z}
$$

and

$$
U\left(d_{t}\right)|p \lambda\rangle_{h}=U\left(R(\hat{p}) R(\pi, \hat{y}) Z\left(x_{p}\right)\right)|\dot{p} \lambda\rangle_{h}
$$


Chapter 3

RELATIVISTIC TWO-PARTICLE STATES WITH SPIN

The Hilbert space representation of any system of noninteracting particles is simply the direct product of the appropriate single-particle Hilbert spaces, and the basis set of state vectors for the multiple-particle Hilbert space can be chosen as the direct product of the single-particle bases. Other bases can, however, be considered.

In this chapter several bases of the two-particle Hilbert space are developed by appealing to the concept of the fundamental state vector and the corresponding construction of the basis state vectors for the single-particle Hilbert space (Chapter 2). The kinematics of a relativistic two-particle system are discussed first with a view towards expressing the system variables in terms of over-all quantities (e.g., total momentum) and internal quantities (e.g., relative momentum). These ideas are applied in $\$ 3.2$ to the 
construction of a variety of bases for the two-particle Hilbert space. In $\$ \$ 3.3$ and 3.4 , the "total-angular-momentum" bases of this Hilbert space are defined, and the Lorentz and improper transformation properties of the constituent basis state vectors are discussed. Many of the more tedious proofs have been relegated to Appendix $C$.

\subsection{The Kinematics of Two-Particle States}

Consider two particles with three-momenta $\vec{K}_{1}$ and $\vec{K}_{2}$ and energies $\varepsilon_{1} \equiv\left(\vec{K}_{1}{ }^{2}+m_{1}{ }^{2}\right)^{\frac{1}{2}}$ and $\varepsilon_{2} \equiv\left(\vec{k}_{2}{ }^{2}+m_{2}{ }^{2}\right)^{\frac{3}{2}}$. Eq. 1-31 implies that the four-momentum of each particle $\left(k_{i} \equiv\left(\varepsilon_{i}, \vec{k}_{i}\right)\right)$ in the reference frame where $\vec{K}_{1}+\vec{K}_{2}=\vec{K}$ is related to the four-momentum in the centerof-mass (CM) reference frame (i.e., that frame where $\vec{K}_{1}+\vec{k}_{2}=0$ ) by

$$
\begin{aligned}
& \vec{k}_{i}=\vec{k}_{i}^{\prime}+\gamma \vec{\beta}\left(\frac{\gamma}{\gamma+1} \vec{\beta} \cdot \vec{k}_{i}^{\prime}+\varepsilon_{i}^{\prime}\right) \\
& \varepsilon_{i}=\gamma\left(\varepsilon_{i}^{\prime}+\vec{\beta}^{\prime} \cdot \vec{k}_{i}^{\prime}\right) \quad: \gamma \equiv\left(1-\vec{\beta}^{2}\right)^{-\frac{3}{2}}
\end{aligned}
$$

where the prime denotes quantities in the $C M$ reference frame. The velocity of the Lorentz transformation, $\vec{\beta}$, is determined by noting that $\vec{K}^{\prime} \equiv \vec{K}_{1}^{\prime}+\vec{K}_{2}^{\prime}$ is zero; so that, $\vec{K}=\vec{B}_{\gamma} E^{\prime}$ and $E=\gamma E^{\prime}$, where $E \equiv \varepsilon_{1}+\varepsilon_{2}$ and $E^{\prime} \equiv \varepsilon_{1}^{\prime}+\varepsilon_{2}^{\prime}$. Hence, 


$$
\vec{\beta}=\frac{\vec{K}}{\vec{E}} \quad \text { and } \quad \gamma=\frac{E}{E} \text {, }
$$

The "relative three-momentum", $\vec{p}$, is defined to be the momentum of particle 1 in the CM reference frame $\left(\overrightarrow{\mathrm{p}} \equiv \overrightarrow{\mathrm{K}}_{1}^{\prime}=-\overrightarrow{\mathrm{K}}_{2}^{\prime}\right)$. The following notation for $C M$ quantities will be used throughout this dissertation:

$$
\begin{aligned}
& \vec{k}_{1} \equiv \vec{k}_{1}^{\prime}=\vec{p} \quad \vec{k}_{2} \equiv \vec{k}_{2}^{\prime}=-\vec{p} \\
& w_{1} \equiv \varepsilon_{1}^{\prime}=\left(\vec{p}^{2}+m_{1}{ }^{2}\right)^{\frac{1}{2}} \quad w_{2} \equiv \varepsilon_{2}^{\prime}=\left(\vec{p}^{2}+m_{2}{ }^{2}\right)^{\frac{1}{2}} \\
& \text { and } \quad w \equiv w_{1}+w_{2}=\left(E^{2}-\vec{k}^{2}\right)^{\frac{1}{2}}
\end{aligned}
$$

With this notation, the three-momenta $\vec{K}_{1}$ and $\vec{k}_{2}$ are

$$
\text { and } \begin{aligned}
\vec{k}_{1} & =\vec{p}+\frac{\vec{k}}{w}\left(\frac{\vec{k} \cdot \vec{p}}{E+w}+w_{1}\right) \\
& \vec{k}_{2}=-\vec{p}+\frac{\vec{k}}{w}\left(-\frac{\vec{k} \cdot \vec{p}}{E+w}+w_{2}\right)
\end{aligned}
$$

These equations can then be inverted to obtain the relative threemomentum $\vec{p}$ in terms of $\overrightarrow{\mathrm{K}}_{1}$ and $\overrightarrow{\mathrm{K}}_{2}(\S \mathrm{C}-1)$.

$$
\vec{p}=\frac{1}{E+w}\left[\left(\varepsilon_{2}+w_{2}\right) \vec{k}_{1}-\left(\varepsilon_{1}+w_{1}\right) \vec{K}_{2}\right]
$$

In the nonrelativistic limit, this reduces to the usual definition of the relative three-nomentum. 


$$
\vec{p} \stackrel{\text { n.r. }}{\longrightarrow} \frac{1}{a_{1}+m_{2}}\left(m_{2} \vec{k}_{1}-m_{1} \vec{k}_{2}\right)
$$

In terms of the total CM energy, $w$, which is also a Lorentz invariant, the magnitude of the relative three-momentum is $(\xi C-2)$

$$
|\vec{p}|=\frac{1}{2 w} \xi\left(w^{2}, m_{1}^{2}, m_{2}^{2}\right)
$$

where $\xi(x, y, z) \equiv\left[(x-y-z)^{2}-4 y z\right]^{\frac{1}{2}}$. (Note: The relative three-momentum that is defined in this section has also been called a "magic vector" by Aaron, et al." Actually, there is nothing very magical about it - except, perhaps, that $|\vec{p}|$ is a Lorentz invariant.)

The above results imply that any function of the threemomenta $\vec{k}_{1}$ and $\vec{k}_{2}$ can be written as a function of $\vec{k}$ and $\vec{p}$. The Jacobian for the variable change $\left(\vec{K}_{1}, \vec{K}_{2}\right)$ to $(\vec{K}, \vec{p})$ is most easily determined by defining the "Wightman-Garding (W-G) relative fourmomentum", $\Delta$, as 54

$$
\begin{aligned}
& \Delta_{0} \equiv \frac{1}{w}\left(w_{2} \varepsilon_{1}-w_{1} \varepsilon_{2}\right) \\
& \searrow \equiv \frac{1}{w}\left(w_{2} \vec{k}_{1}-w_{1} \vec{k}_{2}\right)
\end{aligned}
$$

where $w_{1}, w_{2}$, and $w$ are Lorentz invariants; so that, $\Delta$ is indeed a four-vector. This four-momentum can also be written as 


$$
\Delta=\frac{1}{2}\left(K_{1}-K_{2}\right)-\frac{1}{2 K^{2}}\left(m_{1}^{2}-m_{2}^{2}\right) K
$$

where $K_{1}\left(K_{2}\right)$ is the four-momentum of particle $1(2)\left(K_{i} \equiv\left(\varepsilon_{i}, \vec{k}_{i}\right)\right)$ and $K \equiv K_{1}+K_{2}$. In the $C M$ reference frame, $\Delta_{0}=0$ and $\vec{\Delta}=\vec{p}$.

Eq. 3-9 can now be used to define a W-G relative fourmomentum for arbitrary values of $\left(K_{1}\right)_{0}$ and $\left(K_{2}\right)_{0}$ (i.e., not restricted by the "mass-shel1" condition $\left(K_{i}\right)_{0}=\varepsilon_{i}$, which is a function of $\left.\left|\vec{K}_{i}\right|\right)$. Permitting these additional two degrees of freedon allows one to consider the variable change $\left(\mathrm{K}_{1}, \mathrm{~K}_{2}\right)$ to $(\mathrm{K}, \Delta)$. The Jacobian for this 8-dimensional "phase-space" change is one.

i.e., $\quad d^{4} K_{1} d^{4} K_{2}=d^{4} K d^{4} \Delta$

The Jacobian for the 6-dimensional phase-space change, $\left(\overrightarrow{\mathrm{K}}_{1}, \overrightarrow{\mathrm{K}}_{2}\right)$ to $(\overrightarrow{\mathrm{K}}, \overrightarrow{\mathrm{p}})$, is then determined by restricting the values of $\left(K_{1}\right)_{0}$ and $\left(K_{2}\right)_{0}$ to their mass-shell values. 


$$
\begin{aligned}
d^{3} K_{1} d^{3} K_{2} & =d^{4} K_{1} d^{4} K_{2} \delta\left(\left(K_{1}\right)_{0}-\varepsilon_{1}\right) \delta\left(\left(K_{2}\right)_{0}-\varepsilon_{2}\right) \\
& =4 \varepsilon_{1} \varepsilon_{2} d^{4} K_{1} d^{4} K_{2} \delta^{+}\left(K_{1}^{2}-m_{1}{ }^{2}\right) \delta^{+}\left(K_{2}{ }^{2}-m_{2}^{2}\right) \\
& =4 \varepsilon_{1} \varepsilon_{2} d^{4} K d^{4} \Delta \frac{1}{4 w_{1} w_{2}} \delta\left(\left(k_{1}\right)_{0}-w_{1}\right) \delta\left(\left(k_{2}\right)_{0}-w_{2}\right) \\
& =4 \varepsilon_{1} \varepsilon_{2}\left(\frac{w}{2 w_{1} w_{2}}\right) d^{4} K d^{4} p \delta^{+}\left(K^{2}-w^{2}\right) \delta(p) \\
& =\frac{\varepsilon_{1} \varepsilon_{2}}{E} \frac{w}{w_{1} w_{2}} d^{3} K d^{3} p
\end{aligned}
$$

where the relative four-momentum $p$ has been defined as (for notation see $\$ 1.5)$

$$
p \equiv \ell\left(X_{K}, \hat{K}\right)^{-1} \Delta
$$

with $\cosh \chi_{K}=\gamma_{K}, \sinh \chi_{K}=\gamma_{K}\left|\vec{\beta}_{K}\right|, \vec{\beta}_{K} \equiv \frac{\vec{K}}{K_{0}}$ and $\gamma_{K}=\frac{K_{0}}{\left(K_{0}^{2}-\vec{K}^{2}\right)^{\frac{1}{2}}}$ $\ell\left(X_{K}, \hat{K}\right)$ is a real Lorentz transformation $\left(\gamma_{K}\right.$ real and positive) if $K_{0}>|\vec{K}|$. This is indeed true in Eq. 3-11. (Note: The off-massshell relative momentum that is defined in Eg. $3-12$ will be used extensively in Chapters 5 and 6 of this dissertation.) 


\subsection{The Definition of Two-Particle State Vectors}

The two-particle Hilbert space, $\mathcal{H}^{2}$, is defined to be the direct product of two of the single-particle Hilbert spaces $\left(\mathcal{H}^{1}\right)$ that were defined in Chapter 2. Hence, one set of basis state vectors for $\mathcal{H}^{2}$ is simply the direct product of the $\psi^{1}$ basis state vectors.

$$
\left|k_{1} \lambda_{1} ; k_{2} \lambda_{2}\right\rangle \equiv\left|k_{1} \lambda_{1}\right\rangle \otimes\left|k_{2} \lambda_{2}\right\rangle
$$

It is more convenient, however, if a basis for $y^{2}$ is constructed in a manner that is analogous to the construction of the single-particle basis ( $\$ 2.1)$. This new basis effectively allows one to interpret $\not^{2}$ as a single-particle Hilbert space with additional degrees of freedom and will make the distinction between composite particles and elementary particles less vivid.

The state vectors for this basis of $\mathcal{H}^{2}$ are constructed by first considering the two-particle state with three-momenta $\vec{k}_{1}$ and $\vec{k}_{2}$; such that $\vec{k}_{1}+\vec{k}_{2}=0$. The direct product state vector corresponding to this state will be called the "CM state vector" and is denoted by $\mid k_{1} \lambda_{1} ; k_{2} \lambda_{2}{ }^{2} \mathrm{CM}$. The three-momenta for this state can also be written in terms of a single "relative three-momentum", which is defined by $\vec{p} \equiv \vec{k}_{1}=-\vec{k}_{2}$. Fig. 3.1 illustrates this definition and defines the polar angles of $\vec{p}$. 


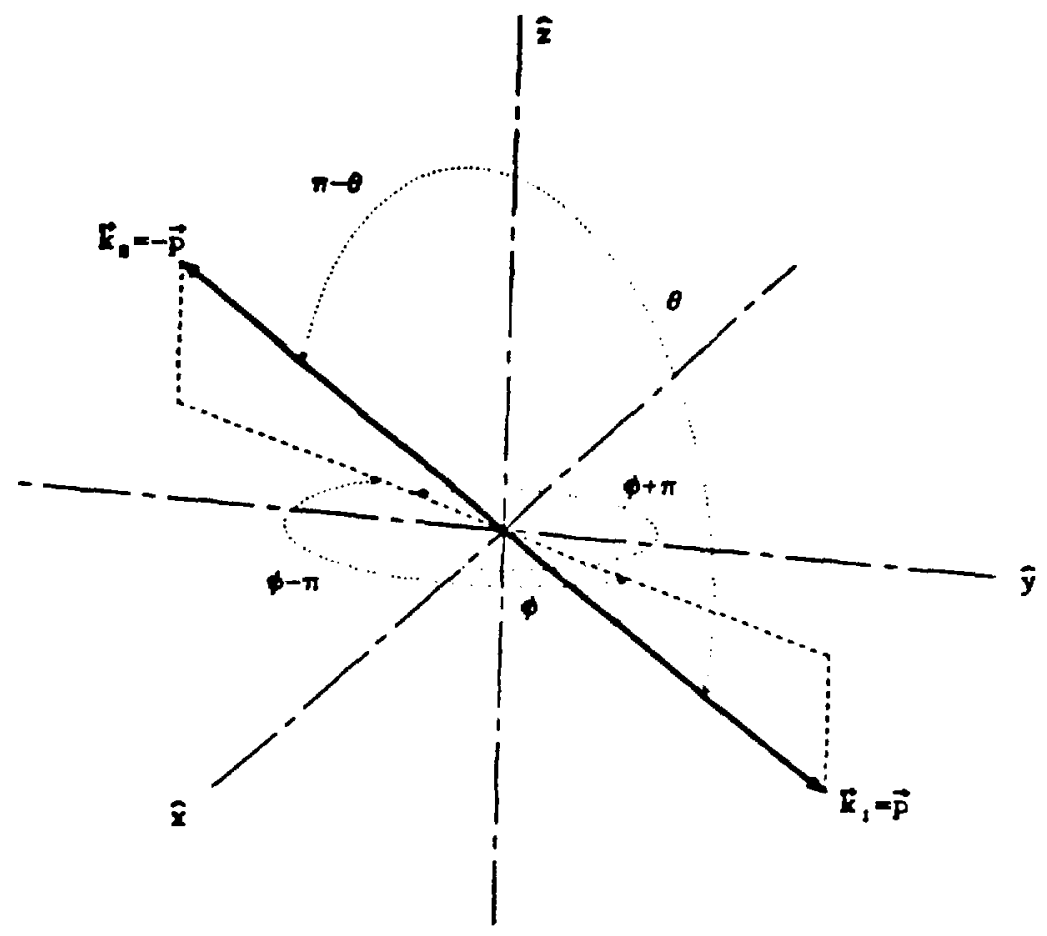

Fig. 3.1 The relative three-momentum $\vec{p}$.

In the 2 basis, the "two-particle fundamental state vector" (cf., \$2.1) will be defined as

$$
\begin{aligned}
& \left.\left.\mid \stackrel{\circ}{(\vec{p}} \lambda_{1} \lambda_{2}\right)_{2}\right\rangle \equiv\left|k_{1} \lambda_{1}\right\rangle \otimes\left|k_{2} \lambda_{2}\right\rangle=\left|k_{1} \lambda_{1} ; k_{2} \lambda_{2}\right\rangle \mathrm{CM} ; z \\
& =U\left(L\left(x_{k_{1}}, \hat{p}\right)\right)\left|k_{1} \lambda_{1}\right\rangle \otimes U\left(L\left(x_{k_{2}},-\hat{p}\right)\right)\left|\hat{k}_{2} \lambda_{2}\right\rangle
\end{aligned}
$$


where $\stackrel{\circ}{K} \equiv k_{1}+k_{2}, \tanh x_{k_{i}}=\frac{|\vec{p}|}{w_{i}}$,

and the other notation is explained in $\$ 2.1 .1$.

In the helicity basis, the two-particle fundamental state vector can be defined to be the direct product of two single-particle state vectors with polar angles $(\phi, \theta)$ for particle 1 and $(\phi+\pi, \pi-\theta)$ for particle 2. (This definition is equivalent to that used by Wick in Ref. 48.)

$$
\begin{aligned}
& \left|\stackrel{0}{ }\left(\vec{p} \lambda_{1} \lambda_{2}\right)_{h}\right\rangle \equiv \mathrm{U}\left(R(\hat{p}) Z\left(x_{k_{1}}\right)\right)\left|\dot{k}_{1} \lambda_{1}\right\rangle \\
& \otimes e^{-i \pi s} 2 U\left(R(\hat{p}) R(\pi, \pi) Z\left(\chi_{k_{2}}\right)\right)\left|\stackrel{\circ}{k_{2}} \lambda_{2}\right\rangle
\end{aligned}
$$

where $\stackrel{\circ}{K} \equiv k_{1}+k_{2}, \quad R(\hat{p}) \equiv R(\phi, \theta) \equiv R(\phi, \hat{z}) R(\theta, \hat{y}), \quad R(\hat{p}) R(\pi, \pi)=R(\phi, \theta)$ $R(\pi, \pi)=R(\phi+\pi, \pi-\theta)$, and the other notation is explained in $\$ 2.1 .2$. The phase factor $\exp \left(-i \pi s_{2}\right)$ has been inserted into this definition for later convenience (e.g., Eq. 3-17). In terms of the CM state vector in the helicity basis (cf., Eq. 2-41),

$$
\left.\stackrel{\circ}{K}\left(\vec{p} \lambda_{1} \lambda_{2}\right)_{h}\right\rangle \equiv \begin{cases}e^{-i \pi s_{2}} \mid k_{1} \lambda_{1} ; k_{2} \lambda_{2}>C M ; h & \text { if } 0 \leqq \phi<\pi \\ e^{+i \pi s_{2}}\left|k_{1} \lambda_{1} ; k_{2} \lambda_{2}\right\rangle \text { CM; } & \text { if } \pi \leqq \phi<2 \pi\end{cases}
$$

The two-particle fundamental state vector in the $Z$ basis is 
related to the two-particle fundamental state vector in the helicity basis by (cf., Eq. 2-22)

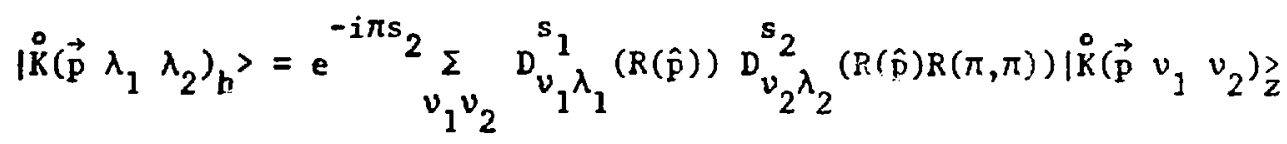

$$
\begin{aligned}
& =\sum_{v_{1} v_{2}} \mathrm{D}_{v_{1} \lambda_{1}}^{s_{1}}(R(\hat{\mathrm{p}})) \mathrm{D}_{v_{2},-\lambda_{2}}^{s_{2}}(R(\hat{\mathrm{p}}))\left|\stackrel{\mathrm{K}}{\mathrm{k}}\left(\overrightarrow{\mathrm{p}} v_{1} v_{2}\right)_{z}\right\rangle
\end{aligned}
$$

This result implies that when $\hat{p}=\hat{z}$, the helicity of particle 1 may be interpreted as its spin projectio: song the $+\hat{z}$ axis; whereas, the helici:y of particle 2 is its spin projection along the $-\hat{z}$ axis. A set of basis state vectors for $\mathcal{H}^{2}$ can now be constructed in exact analogy with the construction of the $\psi^{1}$ basis. In particular a set of basis state vectcrs for $\mathcal{H}^{2}$ can be defined as

$$
\left|K\left(\vec{p} \quad \lambda_{1} \lambda_{2}\right)\right\rangle \equiv U(C(K, \stackrel{\circ}{K}))\left|\stackrel{\circ}{K}\left(\vec{p} \lambda_{1} \lambda_{2}\right)\right\rangle
$$

ie.g., $\left.\left.\left|K\left(\vec{p} \lambda_{1} \lambda_{2}\right)_{2}\right\rangle_{2} \equiv U\left(c_{2}(K)\right) \mid \stackrel{K}{(\vec{p}} \lambda_{1} \lambda_{2}\right)_{2}\right\rangle,\left|K\left(\vec{p} \lambda_{1} \lambda_{2}\right)_{h}\right\rangle_{2} \equiv$ $U\left(C_{z}(K)\right) \mid \stackrel{K}{K}\left(\vec{p} \lambda_{1} \lambda_{2}\right){ }_{h}>$, and so on.) Each of the bases constructed in this manner forms a basis of $\psi^{2}$, and the completeness relation for each basis is determined from Eqs. 2-8, 2-9, and 3-11 (for notation see $\$ 3.1$ ). 


$$
1=\sum_{\lambda_{1} \lambda_{2}} \int \frac{d^{3} K}{2 E} d^{3} p\left(\frac{w}{2 w_{1} w_{2}}\right)\left|K\left(\vec{p} \lambda_{1} \lambda_{2}\right)\right\rangle\left\langle K\left(\vec{p} \lambda_{1} \lambda_{2}\right)\right|
$$

The normalization is

$$
\left\langle K^{\prime}\left(\overrightarrow{p^{\prime}} \lambda_{1}^{\prime} \lambda_{2}^{\prime}\right) \mid K\left(\vec{p} \lambda_{1} \lambda_{2}\right)\right\rangle=2 E \delta^{3}\left(\vec{K}^{\prime}-\vec{k}\right)\left(\frac{2 w_{1} w_{2}}{w}\right) \delta^{3}\left(\vec{p}^{\prime}-\vec{p}\right) \delta_{\lambda_{1}^{\prime} \lambda_{1}} \delta_{\lambda_{2}^{\prime} \lambda_{2}}
$$

\subsection{The Spin of Two-Particle States: Angular-Momentum Expansions}

In $\$ 3.2$, it was shown how to construct two-particle fundamental state vectors ano how to construct bases for $\mathcal{H}^{2}$ in analogy with the construction of the $\mathcal{Y}^{1}$ bases. To complete the analogy, one would like to assign a "spin" to the two-particle state. This is accomplished by using the rotation matrices, which are a complete set of functions over the covering space of $\operatorname{SU}(2)(\$ 1.4)$, to define a new fundamental state vector that rotates as a single-particle spinor. The details of this procedure are discused in $\$ \S 3.3 .1$ and 3.3.2. In \$3.3.3, a basis that has particle 1 interchanged with particle 2 (i.e., $\vec{p}=\vec{k}_{2}=-\vec{k}_{1}$ ) is presented. 


\subsubsection{Basis}

A fundamental state vector in the "orbital-angular nomentum" basis is defined as

$$
\left.\mid \stackrel{\circ}{K}\left(\mathrm{p} \& \text { m } \lambda_{1} \lambda_{2}\right)_{2}\right\rangle \equiv\left(\frac{2 \ell+1}{4 \pi}\right)^{\frac{1}{2}} \int \mathrm{d}^{2} \hat{\mathrm{p}} \mathrm{D}_{\mathrm{mn}}^{\ell}(\mathrm{R}(\hat{\mathrm{p}}))^{\stackrel{\circ}{\mathrm{k}}}\left|\mathrm{K}\left(\overrightarrow{\mathrm{p}} \lambda_{1} \lambda_{2}\right)_{\mathrm{z}}\right\rangle
$$

Conversely,

$$
\left.\left.\left.\mid \stackrel{\mathrm{K}}{\overrightarrow{\mathrm{p}}} \lambda_{1} \lambda_{2}\right)_{2}\right\rangle=\sum_{\ell m}\left(\frac{2 \ell+1}{4 \pi}\right)^{\frac{1}{2}} \mathrm{D}_{\operatorname{mn}}^{\ell}(R(\hat{\mathrm{p}})) \mid \stackrel{0}{K}\left(\mathrm{p} \& \text { m } \lambda_{1} \lambda_{2}\right)_{2}\right\rangle
$$

where $\mathrm{a}$ is an arbitrary but fixed number, $\int \mathrm{d}^{2} \hat{\mathrm{p}} \equiv \int_{0}^{2 \pi} d \phi \int_{0}^{\pi} \sin \theta d \theta$, and $p \equiv|\vec{p}|$. (Note: In the remainder of this chapter, the notation $p \equiv|\vec{p}|$ will be used unless there is a possibility of confusion with the four-momentum $p_{.}$)

A rotation of the state vector $\stackrel{0}{\mathrm{~K}}\left(\mathrm{p} \& \text { in } \lambda_{1} \lambda_{2}\right)_{2}$, yields

$$
\begin{aligned}
& \mathrm{U}(\mathrm{R})\left|\stackrel{\mathrm{K}}{\mathrm{K}}\left(\mathrm{p} \& \mathrm{\&} \quad \lambda_{1} \lambda_{2}\right)_{z}\right\rangle=\left(\frac{2 \ell+1}{4 \pi}\right)^{\frac{1}{2}} \sum_{v_{1} v_{2}} \int \mathrm{d}^{2} \hat{\mathrm{p}} \mathrm{D}_{\mathrm{mb}}^{\ell}(\mathrm{R}(\hat{\mathrm{p}}))^{\frac{\ell}{*}} \mathrm{D}_{v_{1} \lambda_{1}}^{\mathrm{s}_{1}}(\mathrm{R}) \mathrm{D}_{v_{2} \lambda_{2}}^{\mathrm{s}_{2}}(\mathrm{R}) \\
& \times \sum_{\ell^{\prime} m^{\prime}}\left(\frac{2 \ell^{\prime}+1}{4 \pi}\right)^{\frac{1}{2}} D_{m^{\prime} n}^{\ell^{\prime}}(R(r \hat{p})) \mid \stackrel{K}{K}\left(p \ell^{\prime} m^{\prime} v_{1} v_{2}\right)_{2}^{\prime}
\end{aligned}
$$

But Eq. 2-39 says that $R(r \hat{p})=R R(\hat{p}) R(\zeta, \hat{z})^{-1}$ (the value of $\zeta$ is not important here); hence, if $\mathrm{n}$ is set equal to zero (which also implies that $\ell$ is an integer), 


$$
\begin{aligned}
& U(R)\left|\stackrel{\circ}{K}\left(p \quad \& m \lambda_{1} \lambda_{2}\right)_{2}\right\rangle= \\
& v_{1}^{v_{2} m^{\prime}} \mathrm{D}_{m^{\prime} m}^{2}(\mathrm{R}) \mathrm{D}_{v_{1} \lambda_{1}}^{s_{1}}(\mathrm{R}) \mathrm{D}_{v_{2} \lambda_{2}}^{s_{2}}(\mathrm{R})\left|\stackrel{\circ}{\mathrm{K}}\left(\mathrm{p} \& \mathrm{~m}^{\prime} v_{1} v_{2}\right)_{2}\right\rangle
\end{aligned}
$$

The number of rotation matrices in this result can then be reduced by defining a two-particle fundamental state vector in the "2-total-angular-momentum basis" as

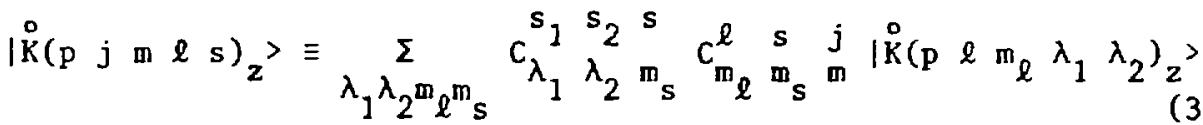
$(3-25)$

where $\quad c_{m_{1}}^{j_{1}} m_{2} j_{2}^{j}$ is a Clebsch-Gordan coefficent. ${ }^{41}$ Under a rotjtion, this state vector transforms as

$$
U(R)\left|\stackrel{0}{K}(p \quad j m \& s)_{z}\right\rangle=\sum_{m}, D_{m \cdot m}^{j}(R)\left|\stackrel{0}{K}(p j m \& s)_{z}\right\rangle
$$

Thus, the state vector $\left.I_{K}^{D}(\mathrm{p} j \mathrm{~m} \quad \ell \quad s)_{z}\right\rangle$ behaves exactly like a single-particle state vector with mass $w$, spin $j$, and spin projection $m$. The state vector labels $\mathcal{L}$ and $s$ play the role of internal quantum numbers. Furthermore, under the action of any proper Lorentz transformation the results of $\$ 2.2$ can be employed without modification. The improper transformations will be discussed in $\S 3.4$.

The completeness relation for this basis is determined from Eq. 3-19 and the normalization is determined from Eq. 3-20. 


$$
1=\sum_{\ell s j m} \int \frac{d^{3} K}{2 F} p^{2} d p\left(\frac{w}{2 w_{1} w_{2}}\right) \mid K(p j m \& s)_{z}>\left\langle K(p j m \& s)_{z}\right|
$$

and

$$
\begin{aligned}
& \left\langle K^{\prime}\left(p^{\prime} j^{\prime} m^{\prime} \ell^{\prime} s^{\prime}\right)_{z} \mid K(p j m \ell s)_{z}\right\rangle= \\
& 2 E \delta^{3}\left(\overrightarrow{\left.K^{\prime}-\vec{K}\right)}\left(\frac{2 w_{1} w_{2}}{w p}\right) \delta\left(p^{\prime}-p\right) \delta_{j^{\prime} j^{2} \delta^{\prime} m^{\prime} \ell^{\prime} \ell^{\prime} s^{\prime} s}\right.
\end{aligned}
$$

where $E \equiv\left(\overrightarrow{\mathrm{K}}^{2}+w^{2}\right)^{\frac{1}{2}}$.

\subsubsection{Helicity Dasis}

The fundamental state vactor in the "helicity-total-angularmomentum basis" is defined as (cf., Eqs. 3-21 and 3-22)

$$
\mid \stackrel{\circ}{K}\left(\mathrm{p} j \mathrm{j} \text { m } \lambda_{1} \lambda_{2}\right)_{\mathrm{h}}>\equiv\left(\frac{2 \mathrm{~J}+1}{4 \pi}\right)^{\frac{2}{2}} \int \mathrm{d}^{2} \hat{\mathrm{p}} \mathrm{D}_{\mathrm{mn}}^{j}(R(\hat{\mathrm{p}}))^{\frac{1}{\hbar}} \mid \stackrel{\circ}{K}\left(\overrightarrow{\mathrm{p}} \lambda_{1} \lambda_{2}\right)>
$$

Conversely,

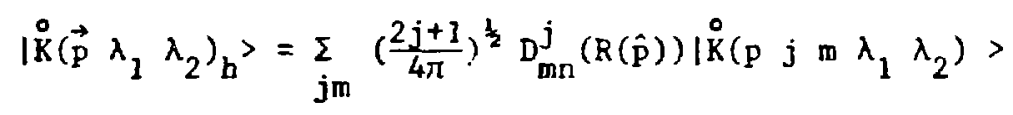

Under a rotation, this state vector transforms as follows: 


$$
\begin{aligned}
& \left.\mathrm{U}(\mathrm{R}) \mid \stackrel{\circ}{\mathrm{K}}\left(\mathrm{p} \text { j m } \lambda_{1} \lambda_{2}\right)_{\mathrm{h}}\right\rangle=\left(\frac{2 j+1}{4 \pi}\right)^{\frac{1}{2}} \underset{v_{1} v_{2}}{\Sigma} \int \mathrm{d}^{2} \hat{\mathrm{p}} \mathrm{D}_{\mathrm{mn}}^{j}(\mathrm{R}(\hat{\mathrm{p}}))^{*} \\
& D_{v_{1} \lambda_{1}}^{s_{1}}\left(R(x \hat{p})^{-1} R R(\hat{p})\right) D_{v_{2} \lambda_{2}}^{s}\left(R(\pi, \pi)^{-1} R(r \hat{p})^{-1} R R(\hat{p}) R(\pi, \pi)\right) \\
& \sum_{j^{\prime} m^{\prime}}\left(\frac{2 j^{\prime}+1}{4 \pi}\right)^{\frac{1}{2}} D_{m^{\prime} n}^{j^{\prime}}(R(r \hat{p})) \mid \grave{K}\left(p j^{\prime} m^{\prime} v_{1} v_{2}\right)_{h^{\prime}} \\
& =\left(\frac{2 j+1}{4 \pi}\right)^{\frac{3}{2}} \sum_{v_{1} \nu_{2}} \int d^{2} \hat{p} D_{m n}^{j}(R(\hat{p})) D_{v_{1} \lambda_{1}}^{s_{1}}(R(\zeta, \hat{z})) D_{-v_{2},-\lambda_{2}}^{s_{2}}(R(\zeta, \hat{z})) \\
& \sum_{j^{\prime} m^{\prime}} D_{m^{\prime} n}^{j^{\prime}}\left(R R(\hat{p}) R(\zeta, \hat{z})^{-1}\right) \mid \hat{K}\left(p j^{\prime} m^{\prime} v_{1} v_{2}\right)_{h}{ }^{\prime}
\end{aligned}
$$

Hence, if $n$ is set equal to $\lambda_{1}-\lambda_{2}$,

$$
U(R) \mid \stackrel{\circ}{K}\left(p \quad j \text { m } \lambda_{1} \lambda_{2}\right)_{h}>=\sum_{m^{\prime}} D_{m^{\prime} m}^{j}(R)\left|\stackrel{o}{K}\left(p \quad j m^{\prime} \lambda_{1} \lambda_{2}\right)_{h}\right\rangle
$$

Note that no Clebsch-Gordan coefficients appear in the definition of this basis. The completeness and normalization relations for this basis are determined as in Eqs. 3-27 and 3-28.

The relation beteen the $\mathrm{Z}$-total-angular-momentum basis and the helicity-total-angular-momentum basis is determined from Eq. 3-17. 
$\left|K\left(p j m \lambda_{1} \lambda_{2}\right)_{h}\right\rangle=\sum_{\ell s}\left(\frac{2 \ell+1}{2 j+1}\right)^{\frac{l}{2}} c_{\lambda_{1}}^{s}-\lambda_{2} \wedge c_{0} \wedge \wedge\left|K(p j m \& s)_{2}\right\rangle$

where $\Lambda \equiv \lambda_{1}-\lambda_{2}$.

\subsubsection{Permutations}

I. the definition of the two-particle fundamental state vector (Eqs. 3-14 and 3-15), the three-momentum $\overrightarrow{+p}$ was assigned to

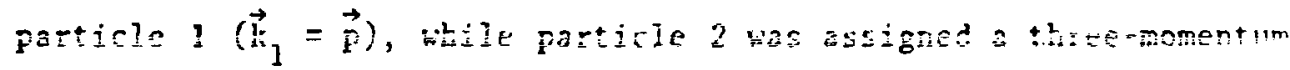
equal to $-\vec{p}\left(\vec{k}_{2}=-\vec{p}\right)$. An equally viable basis of $z^{2}$ is obtained by interchanging particle 1 and particle 2 in these definitions.

In the $Z$ basis, the two-particle fundamental state vector obtained from this interchange is

$$
\begin{aligned}
& \left.\mid K\left(\vec{p} \lambda_{2} \lambda_{1}\right)\right)_{z}>\equiv v\left(L\left(x_{k_{2}}, \hat{p}\right)\right)\left|{ }_{k} \lambda_{1}>\otimes U\left(L\left(x_{k_{1}},-\hat{p}\right)\right)\right| k_{2} \lambda_{2}> \\
& =\left|\stackrel{\circ}{K}\left(\begin{array}{lll}
-\vec{p} & \lambda_{1} & \lambda_{2}
\end{array}\right)_{z}\right\rangle
\end{aligned}
$$

Hence $(\$ C-3)$,

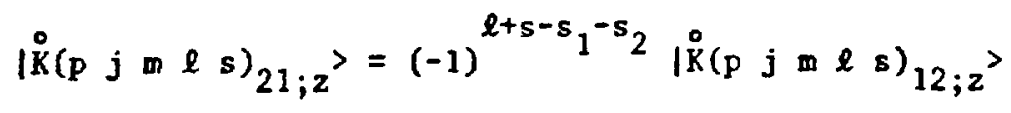

where the state vector subscripted by 12 is defined in $\$ 3.2$ and the 
state vector subscripted by 21 is derived from the fundamental state vector in Eq. 3-33.

In the helicity basis, the two-particle fundamental state vector obtained by interchanging particle 1 and particle 2 is

$$
\begin{aligned}
\left|\stackrel{o}{K}\left(\vec{p} \quad \lambda_{2} \lambda_{1}\right)_{h}\right\rangle & \equiv U\left(R(\hat{p}) Z\left(x_{k_{2}}\right)\right)\left|\stackrel{o}{k_{2}} \lambda_{2}\right\rangle \\
& \left.\otimes e^{-i \pi s} 1\right) U\left(R(\hat{p}) R(\pi, \pi) Z\left(x_{k_{1}}\right)\right)\left|\stackrel{o}{k}_{1} \lambda_{1}\right\rangle
\end{aligned}
$$

Hence $(\S C-4)$,

$$
\left.\left.\mid \stackrel{\circ}{K}\left(\text { p j f m } \lambda_{2} \lambda_{1}\right)_{h}\right\rangle=(-1)^{j-s_{1}-s_{2}} \stackrel{\circ}{K}\left(p \text { j m } \lambda_{1} \lambda_{2}\right)_{h}\right\rangle
$$

This result is in agreement with Ref. 47 .

\subsection{Improper Transformations of Two-Particle States}

The improper transformations of the single-particle states were considered in $\$ 2.3$, and the results of that analysis can be used to determine the effect of the space-inversion operator, $U\left(d_{s}\right)$, and the time-reversal operator, $U\left(\mathcal{L}_{t}\right)$, on the two-particle state vectors. The principle ingredient used in the evaluation of these operations is $U(\mathcal{f}) U(I)=U\left(\xi^{*} \xi^{\dagger}\right) U(\mathcal{L})$, where is either of the improper transformations, $\zeta=R(\pi, \hat{y})$, and $L$ is any proper Lorentz transformation (see \$2.3). 
For the space-inversion transformation ( $(C-3)$,

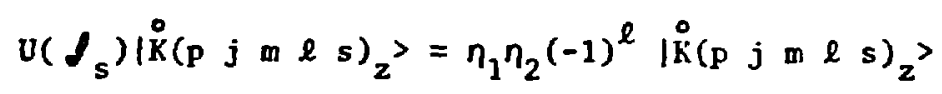

and $(\$ \mathrm{C}-5)$

$$
\left.\mathrm{U}\left(\boldsymbol{d}_{s}\right) \stackrel{\circ}{\mathrm{K}}\left(\mathrm{p} \text { j m } \lambda_{1} \lambda_{2}\right)_{h}\right\rangle=\eta_{1} \eta_{2}(-1)^{j-s_{1}-s_{2}}\left|K\left(\mathrm{p} j \mathrm{~m}-\lambda_{1}-\lambda_{2}\right)\right\rangle
$$

where $\eta_{1}\left(\eta_{2}\right)$ is the parity of particle 1 (2).

Eq. 3-38 suggests that another belicity-total-angularmomentum basis be defined using the following two-particle fundamental state vector:

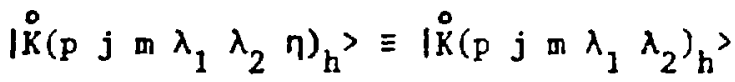

$$
\begin{aligned}
& +\eta \eta_{1} \eta_{2}(-1)^{j-s_{1}-s_{2}} \mid \stackrel{0}{K}\left(p j m-\lambda_{1}-\lambda_{2}\right){ }_{h}>
\end{aligned}
$$

with $\eta= \pm 1$. This state vector is diagonal under the space-inversion operation.

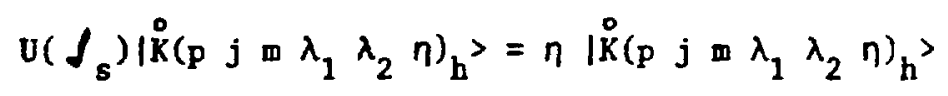

It should be noted, however, that the completeness relation for a basis of $\psi^{2}$ constructed from this two-particle fundamental state vector will involve a restricted summation over the helicity labels (i.e., $\lambda_{1} \geq 0$ only ox $\lambda_{2} \geq 0$ only). 


\section{$s$}

For the time reversal transformation $(\$ C-6)$,

$$
\mathrm{U}\left(\mathscr{d}_{\mathrm{t}}\right)\left|\stackrel{0}{\mathrm{~K}}(\mathrm{p} \mathrm{j} \mathrm{m} \ell \mathrm{s})_{\mathrm{z}}\right\rangle=(-1)^{\mathrm{j}-\mathrm{m}}\left|\stackrel{0}{\mathrm{~K}}(\mathrm{p} \mathrm{j}-\mathrm{m} \ell \mathrm{s})_{z}\right\rangle
$$

and

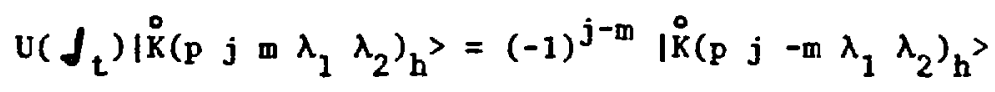

This result is similar to that obtained for the single-particle fundamental state vector (cf., Eq. 2-68). 
Chapter 4

SCATTERING THEORY

A "scattering event" is defined as a transformation from some initial particle configuration to some final particle configuration, where the number and types of particles in the initial and final system can be different. This event can be represented by an operator that maps the initial systen state vector onto the final system state vector within the appropriate multiple-particle Hilbert space. The "scattering amplitude" is the expectation value of this operator between these state vectors.

The Poincaré and improper transformation properties of the scattering amplitude are considered and the reaction matrix (or $R$ natrix) is defined in $\$ 4.1$. The improper transformation properties of the amplitude suggest that the $R$ matrix be considered as a function of the four-momentum of each particle involved in the scattering event, and that another scattering process is associated with 
that function when the signs of those four-momenta are changed. However, this interpretation is complicated by the appearance of kinematic singularities (i.e., singularities that arise from the particular choice of the multiple-particle Hilbert space basis) in the Lorentz transformed function.

This problen is addressed in $\$ 4.2$ by defining a new function (called an "M function") that is related to the $R$ matrix but free of kinematic singularities. The $M$ function that is used in this dissertation is related to Stapp's $M$ function, ${ }^{14}$ but it has certain properties that make the formulation of scattering theory simpler. Having eliminated the kinematic singularities, the improper transformation properties of the $M$ function are then used to suggest the existence of antiparticles and to define the antiparticle scattering amplitude by continuing the $M$ function into regions of negative energy.

The off-mass-shell scattering amplitude is defined in $\$ 4.3$ through a consideration of a time-ordered sequence of scattering events and the $M$ function formalism. This leads to the postulation of a set of Feynman rules for a dynamical theory in terms of the $M$ function. This theory is essentially equivalent to a perturbative, field-theoretic treatment of the scattering problem (see Appendix D); but, the $M$ function formalism, which is developed here, has the following distinct advantages: 
1) Particles with arbitrary spin are included from the start. Additional complications that arise for high-spin particles in field theory (e.g., uncertainty as to the particular representation of the Lorentz group that is used to represent the fields - cf., Weinberg ${ }^{28}$ and Appendix D) are not encountered.

2) Equations that are generated by this method have the same form as the equations of a nonrelativistic potential theory and can be interpreted as a relativistic, off-mass-shell generalization of such a theory.

3) This formalism is oasily subjected to reduejon techriques that allow one to define off-energy-shell scattering amplitudes and make a connection to analytic $S$ matrix theory. (This aspect of the formalism will be discussed in later chapters.)

The properties of a space-inversion symmetric, time-reversal symmetric, and charge-conjugate symmetric $M$ function are established in \$4.4. Combining these symmetries gives rise to a CPI theorem in terms of the $M$ function, which is satisfied provided the $M$ function satisfies certain analytic requirements.

An $M$ function for the $\pi N N$ vert.ex is derived in $\$ 4.5$ from the field-tbeoretic, pseudo-scalar interaction Hamiltonian. This example and the results of Appendix $D$, which is a review of a fieldtheoretic formalism that is similar to the formalism presented here, provide a basis for many of the suppositions that are made in the development of the $M$ function formalism. 


\subsection{The S Matrix and the R Matrix}

Consider a scattering event where particles $1,2, \cdots$, n collide and result in a set of outgoing particles $1^{\prime}, 2^{\prime}, \cdots, n^{\prime}$. Within the appropriate multiple-particle Hilbert space, this event is represented by a unitary operator $3^{13-15}$ The expectation value of $S$ between the final state vector and the initial state vector, $\left.<k_{1}^{\prime} \lambda_{1}^{\prime} \cdots k_{n}^{\prime} \lambda_{n}^{\prime}|s| k_{1} \lambda_{1} \cdots k_{n} \lambda_{n}\right\rangle$, is called the "scattering amplitude", and the probability that the event will occur is proportional to $\left|<k_{i}^{\prime} \lambda_{i} \cdots k_{i}^{\prime} \lambda_{u}^{\prime} ! s\right| k_{1} \lambda_{1} \cdots k_{i} \lambda_{u}>\left.\right|^{2}$. The set of ail possible scattering amplitudes is called the "S matrix". If no scattering occurs $S=1$ and the scattering amplitude is zero unless the final state is the same as the initial state. Hence, the actual reaction amplitude is determined by the reaction operator, $\mathrm{R} \equiv \mathrm{S}-\mathrm{l}$.

A translation of the scattering event by a space-time distance $x$ results in a phase change of the reaction amplitude.

$$
\begin{aligned}
& P(x, 1):\left\langle k^{\prime} \lambda^{\prime}|R| k \lambda\right\rangle \rightarrow\left\langle k^{\prime} \lambda^{\prime}|R| k \lambda\right\rangle_{x} \\
& \left.\equiv U(P(x, 1)) \psi_{k} \cdot \lambda^{\prime}, R U(P(x, 1)) \psi_{k \lambda}\right) \\
& \left.=e^{-i K^{\prime} \cdot x+i K \cdot x}<k^{\prime} \lambda^{\prime}|R| k \lambda\right\rangle
\end{aligned}
$$

In this relation, $|k \lambda\rangle$ is an abbreviated notation for the multiple-particle state vector, $|k \lambda\rangle \equiv\left|k_{1} \lambda_{1} \cdots k_{n} \lambda_{n}\right\rangle$, and $k$ is the 
total four-momentum, $K \equiv k_{1}+\cdots+k_{n}$. This notation will be used throughout this chapter.

Under a Lorentz transformation, the reaction amplitude is determined from the results of Chapter 2 .

$$
\begin{aligned}
& P(0, I):\left\langle k^{\prime} \lambda^{\prime}|R| k \lambda\right\rangle \rightarrow\left\langle k^{\prime} \lambda^{\prime}|R| k \lambda\right\rangle_{I} \\
& \equiv\left(U(L) \psi_{k^{\prime} \lambda^{\prime}}, R U(L) \psi_{k \lambda}\right) \\
& =\sum_{v^{\prime} v} D_{\lambda^{\prime} v^{\prime}}^{S^{\prime}}\left(R\left(L ; k^{\prime}\right)^{\dagger}\right)\left\langle\ell k^{\prime} v^{\prime}|R| \ell k v\right\rangle D_{v \lambda}^{S}(R(L ; k))
\end{aligned}
$$

$R(L ; p)=C(\ell p)^{-1} L C(p)(c f ., \$ 2.2)$ and a rotation matrix for each final-state and initial-state particle is implied.

Improper transformations were also considered in Chapter 2. Under a space-inversion transformation, the new state vector is given by $\mathrm{Eq}_{\mathrm{q}}$ 2-53 and the new reaction amplitude is given by

$$
\begin{aligned}
d_{s} & :\left\langle k^{\prime} \lambda^{\prime}|R| k \lambda\right\rangle \rightarrow\left\langle k^{\prime} \lambda^{\prime}|R| k \lambda\right\rangle_{P} \equiv\left(U\left(d_{s}\right) \psi_{k^{\prime}} \lambda^{\prime}, R U\left(d_{s}\right) \psi_{k}\right) \\
& =\eta_{p} \sum_{v^{\prime} v} D_{\lambda^{\prime} v^{\prime}}\left(R_{s}\left(k^{\prime}\right)^{\dagger}\right)\left\langle I_{s^{\prime}} k^{\prime} v^{\prime}|R| I_{s} k v\right\rangle D_{v \lambda^{s}}\left(R_{s}(k)\right) \quad(4-3)
\end{aligned}
$$

where $R_{s}(k)=C\left(I_{s} k\right)^{-1} \zeta C(k)^{*} \zeta^{\dagger}$ and $\eta_{p}$ is the product of all the final-state and initial-state particle parities. Under a timereversal transformation $(\$ 2.3 .2)$, the new reaction amplitude is given by 


$$
\begin{aligned}
\mathcal{d}_{t}: & \left\langle k^{\prime} \lambda^{\prime}|R| k \lambda\right\rangle \rightarrow\left\langle k^{\prime} \lambda^{\prime}|R| k \lambda\right\rangle_{T} \equiv\left(U\left(\mathcal{L}_{t}\right) \psi_{k^{\prime} \lambda^{\prime}}, R U\left(\mathcal{L}_{t}\right) \psi_{k \lambda^{\prime}}\right) \\
& =\sum_{v^{\prime} v} D_{\lambda^{\prime} v^{\prime}}^{s^{\prime}}\left(\zeta^{\dagger} R_{s}\left(k^{\prime}\right)^{\dagger}\right)\left\langle I_{s} k^{\prime} v^{\prime}|R| I_{s} k v\right\rangle D_{v \lambda^{s}}^{s}\left(R_{s}(k) \zeta\right)
\end{aligned}
$$

And, under the combined operation $\left(f_{s} f_{t}\right)$, the reaction amplitude is given by

$$
\begin{aligned}
f_{s} f_{t}:\left\langle k^{\prime}\right. & \left.\lambda^{\prime}|R| k \lambda\right\rangle \rightarrow\left\langle k^{\prime} \lambda^{\prime}|R| k \lambda\right\rangle_{P T} \\
& =\eta_{p} \sum_{v^{\prime} v} D_{\lambda^{\prime} v^{\prime}}^{s^{\prime}}\left(\zeta^{\dagger}\right)\left\langle k^{\prime} v^{\prime}|R| k v\right\rangle D_{v \lambda}^{s}(\zeta)
\end{aligned}
$$

Also note that

$\left\langle k^{\prime} \lambda^{\prime}|R| k \lambda\right\rangle_{T}=\eta_{P} \sum_{v^{\prime} v} D_{\lambda v^{s}}^{s}\left(R_{s}(k)^{\dagger}\right)\left\langle I_{s^{\prime}} k^{\prime} v^{\prime}|R| I_{s} k v\right\rangle_{P T} D_{v^{\prime} \lambda^{\prime}}^{s^{\prime}}\left(R_{s}\left(k^{\prime}\right)\right)$

Eq. 4-5 implies that the reaction amplitude $\left\langle k^{\prime} \lambda^{\prime}|R| k \lambda\right\rangle_{P T}$ translates as in Eq. 4-1, but it Lorentz transforms as if the final state particles were in the initial state and vice versa.

$$
\text { i.e., } \begin{aligned}
& P(x, I):\left\langle k^{\prime} \lambda^{\prime}|R| k \lambda\right\rangle_{P T} \rightarrow\left\langle k^{\prime} \lambda^{\prime}|R| k \lambda\right\rangle_{(x, I) P T} \\
= & e^{-i K^{\prime} \cdot x+i K \cdot x} \sum_{v^{\prime} v} D_{\lambda v^{s}}^{s}\left(R(L ; k)^{\dagger}\right)\left\langle l k^{\prime} v^{\prime}|R| l k v\right\rangle_{P T} D_{v^{\prime} \lambda^{\prime}}^{s^{\prime}}(R(L ; k))
\end{aligned}
$$

(see Eq. 2-50).

This property suggests that the amplitude $\left\langle k^{\prime} \lambda^{\prime}|R| k \lambda\right\rangle_{P T}$ be considered as a function of the four-momentum of each particle 
(i.e., $\left\langle k^{\prime} \lambda^{\prime}|R| k \lambda\right\rangle_{\mathrm{PT}} \equiv \mathrm{R}_{\mathrm{PT}}\left(\mathrm{k}^{\prime} \lambda^{\prime} ; k \lambda\right)$ ), and that this function is associated with another scattering process. This new scattering process would be defil: d by associating an "antiparticle" (denoted by $1_{c}, 2_{c}, \cdots$ ) with each particle, such that the reaction amplitude for the process $1_{c}+\cdots+n_{c}^{\prime} \rightarrow 1_{c}+\cdots+n_{c}$ (i.e., $\left.\left\langle(k \lambda)_{c}|R|\left(k^{\prime} \lambda^{\prime}\right)_{c}\right\rangle\right)$ is equal to the function $R_{P T}\left(-k^{\prime} \lambda^{\prime} ;-k \lambda\right)$. However, the assocation cannot be complete without also considering the Lorentz transformation properties of the function $R_{\mathrm{PT}}\left(k^{\prime} \lambda^{\prime} ; k \lambda\right)$ for negative values of the four-momenta. This implies that the rotation $R(I ; k)=C(\ell k)^{-1} L C(k)$ in $E q .4-7$ must be considered for negative $k$ (i.e., $\varepsilon(|\vec{k}|) \rightarrow-\varepsilon(|\vec{k}|)$ and $\vec{k} \rightarrow-\vec{k})$. But, square-root singularities are encountered when $\varepsilon(|\vec{k}|)$ is changed to $-\varepsilon(|\vec{k}|)$ in $C(k)$. (e.g., if $C(k)=C_{h}(k)=R(\hat{k}) Z\left(x_{k}\right)$ with

$$
z\left(x_{k}\right)=\left(\frac{\varepsilon+m}{2 m}\right)^{\frac{3}{2}} \sigma_{0}-\left(\frac{\varepsilon-m}{2 m}\right)^{\frac{3}{2}} \sigma_{3}
$$

and $\varepsilon=\left(|\vec{k}|^{2}+m^{2}\right)^{\frac{1}{2}}$, then the sign change $\varepsilon \rightarrow-\varepsilon$ requires a complex interpretation of the square root factors with branch points in the function $\mathrm{Z}(X)$ at $\varepsilon=+m$ and $\varepsilon=-m$.) Moreover, these square-root singularities are dependent on the particular choice of the complementary set of Lorentz transformations. (That is, they are dependent on the particular basis set of state vectors that is chosen for the aultiple-particle Hilbert space.) As such, these singulari- 
ties, which are called "kinematic singularities", are not a dynamical property of the system being studied, but are an artifice caused by the choice of the Hilbert space representation of the scattering event. ${ }^{14,18}$ Thus, if the association of particles to antiparticies is to be complete, a new function that is free of kinematic singularities (i.e., basis independent) must be defined.

\subsection{The M Functions}

The $M$ function was first introduced by Stapp $^{14}$ as a means of eliminating the kinematic singularities that were encountered in §4.1. Stapp defined the $M$ function as

$$
\bar{M}\left(k^{\prime} \lambda^{\prime} ; k \lambda\right) \equiv \sum_{v^{\prime} v} D_{\lambda^{\prime} v^{\prime}}^{s^{\prime}}\left(C\left(k^{\prime}\right)\right)<k^{\prime} v^{\prime}|R| k v>D_{v \lambda}^{s}\left(C(k)^{\dagger}\right)
$$

With this definition, Stapp's $M$ function has the Lorentz transformation property:

$$
\begin{aligned}
\rho(0, L): \bar{M}\left(k^{\prime} \lambda^{\prime} ; k \lambda\right) \rightarrow \bar{M}_{L}\left(k^{\prime} \lambda^{\prime} ; k \lambda\right) \\
=\sum_{v^{\prime} v} D_{\lambda^{\prime} v^{\prime}}^{s^{\prime}}\left(L^{-1}\right) \bar{H}\left(2 k^{\prime} v^{\prime} ; \ell k v\right) D_{v \lambda^{s}}^{s}\left(\left(L^{-1}\right)^{\dagger}\right)
\end{aligned}
$$

As can be seen in Eq. 4-8, Stapp's M function is defined by removing the problem causing complementary set Lorentz transformation from the $R$ matrix. In field theory, this is equivalent to removing the 
spinors from the reaction amplitude to obtain an n-point function as in the ISZ reduction procedure. A specific example of this will be given in $\$ 4.5$.

In this dissertation, the $M$ function is defined as

$M\left(k^{\prime} \lambda^{\prime} ; k \lambda\right) \equiv \frac{i}{2 \pi} \sum_{v^{\prime} v} D_{\lambda^{\prime} v^{\prime}}^{s^{\prime}}\left(C\left(k^{\prime}\right)\right)\left\langle k^{\prime} v^{\prime}|R| k v\right\rangle D_{v \lambda}^{s}\left(C(k)^{-1}\right)$

which has the Poincaré transformation property

$$
\begin{aligned}
& P(x, L): M\left(k^{\prime} \lambda^{\prime} ; k \lambda\right) \rightarrow M_{(x, L)}\left(k^{\prime} \lambda^{\prime} ; k \lambda\right) \\
& =e^{-i K^{\prime} \cdot x+i K \cdot x} \sum_{v^{\prime} v} D_{\lambda^{\prime} v^{\prime}}^{s^{\prime}}\left(L^{-1}\right) M\left(l k^{\prime} v^{\prime} ; \ell k v\right) D_{v \lambda}^{s}(I)
\end{aligned}
$$

This M function is related to Stapp's M function by

$$
M\left(k^{\prime} \lambda^{\prime} ; k \lambda\right)=\frac{i}{2 \pi} \sum_{v} \bar{M}\left(k^{\prime} \lambda^{\prime} ; k v\right) D_{v \lambda}^{s}\left(\frac{\tilde{\sigma} \cdot k}{m}\right)
$$

where (see Eq. 1-39) $\quad C(k) C(k)^{\dagger}=\frac{1}{m} C(k) \sigma \cdot \frac{i}{k} C(k)^{\dagger}=\frac{\sigma \cdot k}{m}$

and $\left(\frac{\sigma \cdot k}{m}\right)^{-1}=\frac{\tilde{\sigma} \cdot k}{m} \quad: \quad \tilde{\sigma} \equiv\left(\sigma_{0},-\vec{\sigma}\right)$

and the factor $\frac{i}{2 \pi}$ has been inserted for later convenience: This definition of the $M$ function will allow for a simple statement of the dynamical scattering theory in $\$ 5.4$. 
In a similar manner, $M_{p}\left(k^{\prime} \lambda^{\prime} ; k \lambda\right)$ is defined from Eq. 4-3.

$$
\begin{gathered}
M_{P}\left(k^{\prime} \lambda^{\prime} ; k \lambda\right)=\eta_{p} \frac{i}{2 \pi} \sum_{v^{\prime} v} D_{\lambda^{\prime} v^{\prime}}^{s^{\prime}}\left(C\left(k^{\prime}\right)\right)\left\langle k^{\prime} v^{\prime}|R| k v\right\rangle_{P} D_{v \lambda}^{s}\left(C(k)^{-1}\right) \\
=\eta_{p} \sum_{v^{\prime} v} D_{\lambda^{\prime} v^{\prime}}^{s^{\prime}}\left(\frac{\sigma^{\circ} k^{\prime}}{m^{\prime}}\right) M\left(I_{s^{\prime}} k^{\prime} v^{\prime} ; I s^{k} v\right) D_{v \lambda}^{s}\left(\frac{\tilde{\sigma} \cdot k}{m}\right)
\end{gathered}
$$

and $M_{\mathrm{PT}}\left(\mathrm{k}^{\prime} \lambda^{\prime} ; \mathrm{k} \lambda\right)$ is defined by appealing to Eq. 4-5 and the Lorentz transformation property that is shown in Eq. 4-7.

$$
\begin{aligned}
& M_{P T}\left(k^{\prime} \lambda^{\prime} ; k \lambda\right)=\eta_{P} \sum_{v^{\prime} v} D_{\lambda^{\prime} v^{\prime}}^{s^{\prime}}\left(\zeta^{\dagger}\right) M\left(k^{\prime} v^{\prime} ; k v\right) D_{v \lambda}^{s}(\zeta) \\
& =\frac{i}{2 \pi} \sum_{v^{\prime} v} D_{\lambda v^{\prime}}^{s}(C(k))\left\langle k^{\prime} v^{\prime}|R| k v\right\rangle_{P T} D_{v^{\prime} \lambda^{\prime}}^{s^{\prime}}\left(C\left(k^{\prime}\right)^{-1}\right)
\end{aligned}
$$

This function has the following Poincare transformation property:

$$
\begin{aligned}
& \sigma(x, L): M_{P T}\left(k^{\prime} \lambda^{\prime} ; k \lambda\right) \rightarrow M_{(x, L) P T}\left(k^{\prime} \lambda^{\prime} ; k \lambda\right) \\
& \left.=e^{-i K^{\prime} \cdot x+i K \cdot x} \sum_{v^{\prime} v} D_{\lambda v^{\left(L^{2}\right.}}{ }^{-1}\right) M_{P T}\left(l k^{\prime} v^{\prime} ; \ell k v\right) D_{v^{\prime} \lambda^{\prime}}(L)
\end{aligned}
$$

The function $M_{\mathrm{T}}\left(k^{\prime} \lambda^{\prime} ; k \lambda\right)$ can then be defined from Eqs. 4-15 and 4-16 (cf., Eq. 4-6). 


$$
\begin{gathered}
M_{T^{\prime}}\left(k^{\prime} \lambda^{\prime} ; k \lambda\right)=\sum_{v^{\prime} v} D_{\lambda^{\prime} v^{\prime}}^{s^{\prime}}\left(\zeta^{\dagger} \frac{\tilde{\sigma} \cdot k^{\prime}}{m^{\prime}}\right) M\left(I_{s^{\prime}} k^{\prime} v^{\prime} ; I_{s^{\prime}} k v\right) D_{v \lambda}^{s}\left(\frac{\tilde{\sigma} \cdot k}{m} \zeta\right) \\
=\frac{i}{2 \pi} \sum_{v^{\prime} v} D_{\lambda v^{s}}^{s}(C(k))\left\langle k^{\prime} v^{\prime}|R| k v{ }_{T} D_{v^{\prime} \lambda^{\prime}}^{s^{\prime}}\left(C\left(k^{\prime}\right)^{-1}\right)\right.
\end{gathered}
$$

Having defined the $M$ function, which is assumed to be free of kinematic singularities, the question of antiparticles that was raised at the end of $\$ 4.1$ can be reconsidered. It is hypothesized that for each particle scattering event, $1+\cdots+\mathbf{n} \rightarrow 1+$ $\cdots+n^{\prime}$, there exists an antiparticle scattering event, $1_{c}^{\prime}+\cdots$ $+n_{c}^{\prime} \rightarrow 1_{c}+\cdots+n_{c}$ that is described by $M\left((k \lambda)_{c} ;\left(k^{\prime} \lambda^{\prime}\right)_{c}\right)$ with

$$
\begin{aligned}
& M\left((k \lambda)_{c} ;\left(k^{\prime} \lambda^{\prime}\right)_{c}\right)=M_{P T}\left(-k^{\prime} \lambda^{\prime} ;-k \lambda\right) \\
& =\eta_{p} \sum_{v^{\prime} v} D_{\lambda^{\prime} v^{\prime}}^{s^{\prime}}\left(\zeta^{\dagger}\right) M\left(-k^{\prime} v^{\prime} ;-k v\right) D_{v \lambda}^{s}(\zeta)
\end{aligned}
$$

It should be noted that this hypothesis assumes that the function $M\left(k^{\prime} \lambda^{\prime} ; k \lambda\right)$ can be continued in the real variables $k_{0}^{\prime}=\varepsilon\left(\left|\vec{k}^{\prime}\right|\right)$ and $k_{0}=\varepsilon(|\vec{k}|)$ to the negative values $k_{0}^{\prime}=-\varepsilon(|\vec{k} \cdot|)$ and $k_{0}=-\varepsilon(|\vec{k}|)$.

\subsubsection{Single-Particle Crossing}

It is ainor extension of the above results to consider the anplitude that results when just one of the initial or fial con- 
stituent single-particle state vectors is subjected to the spaceinversion and/or time-reversal operations. In this case, the following relation is obtained:

$$
\begin{aligned}
& M\left(k_{1}^{\prime} \lambda_{1}^{\prime} \cdots\left(k_{i}^{\prime} \lambda_{i}^{\prime}\right)_{P T} \cdots k_{n}^{\prime} \lambda_{n}^{\prime} ; k_{1} \lambda_{1} \cdots k_{n} \lambda_{n}\right) \\
= & \eta_{i}^{\prime} \sum_{v_{i}^{\prime}} D_{\lambda_{i}^{\prime} v_{i}^{\prime}}^{s_{i}^{\prime}}\left(\xi^{\dagger}\right) M\left(k_{1}^{\prime} \lambda_{i}^{\prime} \cdots k_{i}^{\prime} \lambda_{j}^{\prime} \cdots k_{n}^{\prime} \lambda_{n}^{\prime} ; k_{1} \lambda_{1} \cdots k_{n} \lambda_{n}\right)
\end{aligned}
$$

And, the antiparticle hypothesis is

$$
\begin{gathered}
M\left(k_{1}^{\prime} \lambda_{1}^{\prime} \cdots k_{i-1}^{\prime} \lambda_{i-1}^{\prime}, k_{i+1}^{\prime} \lambda_{i+1}^{\prime} \cdots k_{n}^{\prime} \lambda_{n}^{\prime} ; k_{1} \lambda_{1} \cdots k_{n} \lambda_{n},\left(k_{i}^{\prime} \lambda_{i}^{\prime}\right)_{c}\right) \\
=M\left(k_{1}^{\prime} \lambda_{1}^{\prime} \cdots\left(-k_{i}^{\prime} \lambda_{i}^{\prime}\right)_{P T} \cdots k_{n}^{\prime} \lambda_{n}^{\prime} ; k_{1} \lambda_{1} \cdots k_{n} \lambda_{n}\right) \quad(4-21)
\end{gathered}
$$

This property of the M-function will be called its "single-particle crossing" property and is a statement of the equality of the $M$ functions when a particle in the final state is "crossed over" into its corresponding antiparticle in the initial state. A similar result holds for crossing a particle from the initial state to its corresponding antiparticle in the final state (cf., Eq. 4-19).

The existance of antiparticles is, of course, well established fact of nature. The above procedures can be viewed as either a fundamental hypothesis concerning antiparticle scattering amplitudes (i.e., as essentially defining what an antiparticle is) or as a convenient way of obtaining the correct spin relations between the

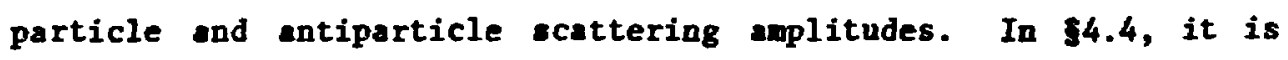


shown that the CPT theorem follows directly from the hypothesis. In the next section, however, the concepts that have evolved during the present analysis will be extended to a consideration of off-massshell scattering functions.

\subsection{View From a Time-Ordered World}

In order to establish a connection between the scattering formalism presented in $\$ \$ 4.1$ and 4.2 and time-dependent scattering theory (e.g., Refs. 6 and 23-26), a reaction operator "density", $R\left(x_{1}, \cdots, x_{n} ; x_{1}, \cdots, x_{n}\right)$, will be defined by the relations:

$$
R \equiv \int d^{4} x_{1}, \cdots d^{4} x_{n} R\left(x_{1},, \cdots, x_{n}\right)
$$

and $U(O(a, 1))^{\dagger} R\left(x_{1}, \cdots, x_{n}\right) U(\infty(a, 1))=R\left(x_{1},+a, \cdots, x_{n}+a\right)$

It will be assumed that this operator describes the space-time properties of the scattering event.

A consequence of this assumption is that the reaction amplitude contains a total four-momentum conserving $\delta$ function.

$$
\text { i.e., } \begin{aligned}
\left\langle k^{\prime} \lambda^{\prime}|R| k \lambda\right\rangle & =(2 \pi)^{4} \delta^{4}\left(K^{\prime}-K\right) \\
& \times \int d^{4} x_{2}, \cdots d^{4} x_{n}\left\langle k^{\prime} \lambda^{\prime}\left|R\left(0, x_{2}, \cdots, x_{n}\right)\right| k \lambda\right\rangle
\end{aligned}
$$


Hence, a new reaction operator can be defined as

$$
\hat{R} \equiv(2 \pi)^{4} \int d^{4} x_{2}, \cdots d^{4} x_{n} R\left(0, x_{2}, \cdots, x_{n}\right)
$$

so that

$$
\left\langle k^{\prime} \lambda^{\prime}|R| k \lambda\right\rangle=\delta^{4}\left(K^{\prime}-K\right)\left\langle k^{\prime} \lambda^{\prime}|\hat{R}| k \lambda\right\rangle
$$

In the $M$ function formalism, $\hat{M}\left(k^{\prime} \lambda^{\prime} ; k \lambda\right)$ will be associated with the amplitude $\left\langle k^{\prime} \lambda^{\prime}|\hat{R}| \mathbf{k} \lambda\right\rangle$.

Now consider the time-ordered (i.e., causal) sequence of scattering events that is shown in Fig. 4.1.

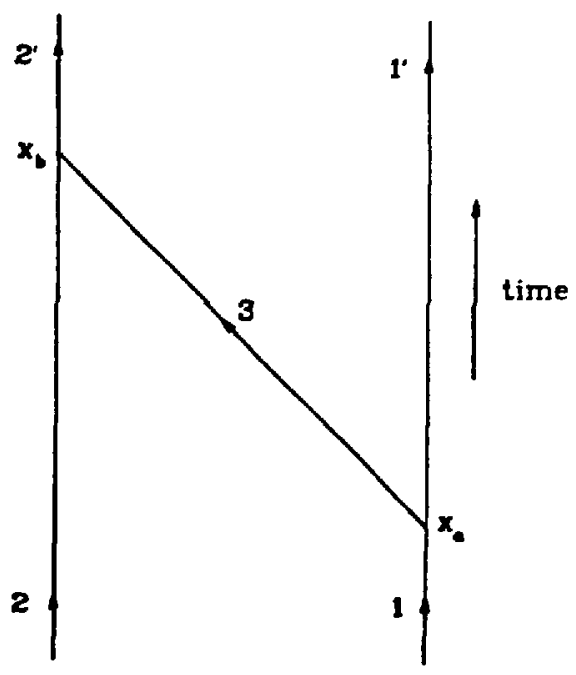

Fig. 4.1 A time-ordered sequence of scattering events. 
The $R$ matrix for this sequence is given by

$$
\begin{aligned}
\left\langle k_{1}^{\prime} \lambda_{1}^{\prime} ; k_{2}^{\prime} \lambda_{2}^{\prime}|R| k_{1} \lambda_{1} ; k_{2} \lambda_{2}\right\rangle=\sum_{\lambda_{3}} \int d^{4} x_{a} d^{4} x_{b} \int \frac{d^{3} k_{3}}{2 \varepsilon_{3}} \theta\left(t_{b}-t_{a}\right) \\
\left.\times(2 \pi)^{-4} e^{-i\left(k_{2}^{\prime}-k_{2}-k_{3}\right) \cdot x_{b}}<k_{2}^{\prime} \lambda_{2}^{\prime}\left|\hat{R}_{b}\right| k_{2} \lambda_{2} ; k_{3} \lambda_{3}\right\rangle \\
\left.\times(2 \pi)^{-4} e^{-i\left(k_{3}+k_{1}^{\prime}-k_{1}\right) \cdot x_{a}}<k_{3} \lambda_{3} ; k_{1} \lambda_{1}\left|\hat{R}_{a}\right| k_{1} \lambda_{1}\right\rangle
\end{aligned}
$$

where $\theta(x)$ is the unit step function $\left(t \equiv x_{0}\right),\left(k_{3}\right)_{0}=\varepsilon_{3} \equiv$ $\left(\vec{k}_{3}{ }^{2}+m_{3}{ }^{2}\right)^{\frac{1}{2}}$, and it has been assumed that the amplitude for $1+2 \rightarrow$ $1^{\prime}+2^{\prime}$ is determined by integrating over all possible emission and absorption points for particle 3 (subject to the restriction that particle 3 must be emitted before it is absorbed). Point-like interactions have also been assumed for simplicity. (Note: If the scattering events were uncorrelated - i.e., if the step function in Eq. 4-27 were removed - then the total reaction amplitude would simply be equal to the product of the two free-particle scattering amplitudes, and four-momentum would be conserved at each vertex.)

The step function can then be rewritten in terms of its Fourier transform,

$$
\theta\left(t_{2}-t_{1}\right)=\frac{i}{2 \pi} \int_{-\infty}^{\infty} d \xi \frac{e^{-i \xi\left(t_{2}-t_{1}\right)}}{\xi+i n}
$$


with the linit $\eta \rightarrow 0+$ taken after the computation is complete, and the $R$ matrices can be replaced by their corresponding $M$ funcions. These replacements yield the following equation for the total M function:

$$
\begin{aligned}
M\left(k_{1}^{\prime} \lambda_{1}^{\prime}, k_{2}^{\prime} \lambda_{2}^{\prime} ; k_{1} \lambda_{1}, k_{2} \lambda_{2}\right) \\
=\sum_{\lambda_{3}} \int d \xi_{3} \frac{d^{3} k_{3}}{2 \varepsilon_{3}} \int d^{4} x_{a} d^{4} x_{b} \frac{1}{\xi_{3}+i \eta} e^{-i \xi_{3}\left(t_{b}-t_{a}\right)} \\
\quad \times(2 \pi)^{-8} e^{-i\left(k_{2}^{\prime}-k_{2}-k_{3}\right) \cdot x_{b}} e^{-i\left(k_{3}+k_{1}^{\prime}-k_{1}\right) \cdot x_{a}} \\
\quad \times \hat{M}_{b}\left(k_{2}^{\prime} \lambda_{2}^{\prime} ; k_{2} \lambda_{2}, k_{3} \lambda_{3}\right) \hat{M}_{a}\left(k_{3} \lambda_{3}, k_{1}^{\prime} \lambda_{1}^{\prime} ; k_{1} \lambda_{1}\right)
\end{aligned}
$$

The integrations over $x_{a}$ and $x_{b}$ can now be performed to obtain

$$
\begin{aligned}
& M\left(k_{1}^{\prime} \lambda_{1}^{\prime}, k_{2}^{\prime} \lambda_{2}^{\prime} ; k_{1} \lambda_{1}, k_{2} \lambda_{2}\right)=\delta^{4}\left(k_{1}^{\prime}+k_{2}^{\prime}-k_{1}-k_{2}\right) \\
& \quad \times \sum_{\lambda_{3}} \int d \xi_{3} \frac{d^{3} k_{3}}{2 \varepsilon_{3}} \frac{1}{\xi_{3}-\varepsilon_{3}+i n} \delta\left(\xi_{3}+\varepsilon_{1}^{\prime}-\varepsilon_{1}\right) \delta^{3}\left(\vec{k}_{3}+\vec{k}_{1}^{\prime}-\vec{k}_{1}\right) \\
& \quad \times \hat{M}_{b}\left(k_{2}^{\prime} \lambda_{2}^{\prime} ; k_{2} \lambda_{2}, k_{3} \lambda_{3}\right) \hat{M}_{a}\left(k_{3} \lambda_{3}, k_{1}^{\prime} \lambda_{1}^{\prime} ; k_{1} \lambda_{1}\right)
\end{aligned}
$$

Note that $k_{3} \equiv\left(\varepsilon_{3}, \vec{k}_{3}\right)$ with $\varepsilon_{3} \equiv\left(\vec{k}_{3}{ }^{2}+m_{3}{ }^{2}\right)^{\frac{3}{2}}$; but, this equation suggests that one consider the replacement $\varepsilon_{3} \rightarrow \xi_{3}$ within the $\hat{M}$ functions. This suggestion is made even more plausible when one recalls that for each particle process there exists an antiparticle process. 
The antiparticle process that corresponds to Fig. 4.1 is shown in Fig. 4.2.

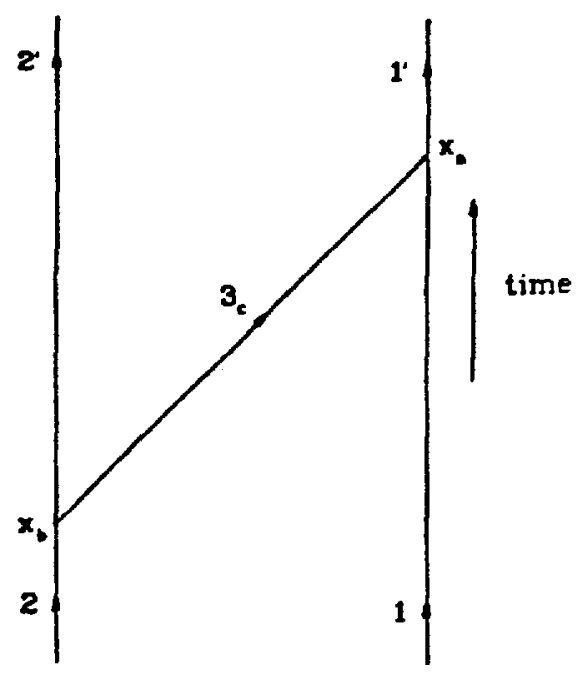

Fig. 4.2 The anti-particle intermediate state.

Eq. 4-19 and the single-particle crossing property of the $M$ function can be used to compute the $M$ function for this process. The result is 


$$
\begin{aligned}
& M\left(k_{1}^{\prime} \lambda_{1}^{\prime}, k_{2}^{\prime} \lambda_{2}^{\prime} ; k_{1} \lambda_{1}, k_{2} \lambda_{2}\right)=\delta^{4}\left(k_{1}^{\prime}+k_{2}^{\prime}-k_{1}-k_{2}\right) \\
& \times \sum_{\lambda_{3}}^{\sum} d \xi_{3} \frac{d^{3} k_{3}}{2 \varepsilon_{3}} \frac{1}{\xi_{3}-\varepsilon_{3}+i \eta} \delta\left(\xi_{3}-\varepsilon_{1}^{\prime}+\varepsilon_{1}\right) \delta^{3}\left(\vec{k}_{3}-\vec{k}_{1}^{\prime}+\vec{k}_{1}\right) \\
& \quad \times \hat{M}_{b}\left(k_{2}^{\prime} \lambda_{2}^{\prime} ; k_{2} \lambda_{2},-k_{3} \lambda_{3}\right) \hat{M}_{a}\left(-k_{3} \lambda_{3}, k_{1}^{\prime} \lambda_{1}^{\prime} ; k_{1} \lambda_{1}\right)
\end{aligned}
$$

Comparing this equation with Eq. 4-30, it is seen that if the replacement $\varepsilon_{3} \rightarrow \xi_{3}$ is made within the $\hat{M}$ functions, which implies a change in the basic structure of the theory, and the variables of integration in Eq. 4-31 are changed, $\xi_{3} \rightarrow-\xi_{3}$ and $\vec{k}_{3} \rightarrow-\vec{k}_{3}$, then these equations can be added together to give

$$
\begin{aligned}
& M\left(k_{1}^{\prime} \lambda_{1}^{\prime}, k_{2}^{\prime} \lambda_{2}^{\prime} ; k_{1} \lambda_{1} k_{2} \lambda_{2}\right)=\delta^{4}\left(k_{1}^{\prime}+k_{2}^{\prime}-k_{1}-k_{2}\right) \\
& \quad \times \sum_{\lambda_{3}} \int d \xi_{3} d^{3} k_{3} \frac{1}{\xi_{3}{ }^{2}-\varepsilon_{3}{ }^{2}+i \eta} \delta\left(\xi_{3}+\varepsilon_{1}^{\prime}-\varepsilon_{1}\right) \delta^{3}\left(\vec{k}_{3}+\vec{k}_{1}-\vec{k}_{1}\right) \\
& \quad \times \hat{M}_{b}\left(k_{2}^{\prime} \lambda_{2}^{\prime} ; k_{2} \lambda_{2}, \xi_{3} \vec{k}_{3} \lambda_{3}\right) \hat{M}_{a}\left(\xi_{3} \vec{k}_{3} \lambda_{3}, k_{1}^{\prime} \lambda_{1}^{\prime} ; k_{1} \lambda_{1}\right)
\end{aligned}
$$

$\xi_{3}$ is now defined to be the energy component of the four-momentum $k_{3}$ (i.e., $\left.\left(k_{3}\right)_{0} \equiv \xi_{3}\right)$ and Eq. 4-32 is rewritten as 


$$
\begin{aligned}
M\left(k_{1}^{\prime} \lambda_{2}, k_{2}^{\prime}\right. & \left.\lambda_{2}^{\prime} ; k_{1} \lambda_{1} k_{2} \lambda_{2}\right)=\delta^{4}\left(k_{1}^{\prime}+k_{2}^{\prime}-k_{1}-k_{2}\right) \\
& \times \sum_{\lambda_{3}} \int d^{4} k_{3} \delta^{4}\left(k_{3}+k_{1}^{\prime}-k_{1}\right) \frac{1}{k_{3}{ }^{2}-m_{3}{ }^{2}+i n} \\
& \times \hat{M}_{b}\left(k_{2}^{\prime} \lambda_{2}^{\prime} ; k_{2} \lambda_{2}, k_{3} \lambda_{3}\right) \hat{M}_{a}\left(k_{3} \lambda_{3}, k_{1}^{\prime} \lambda_{1}^{\prime} ; k_{1} \lambda_{1}\right)(4-33)
\end{aligned}
$$

In this equation $k_{3}^{2} \neq m_{3}^{2}$; hence, particle 3 is said to be offmass-shell.

The extension of the $M$ function to off-mass-shell values of the four-momentum has only been introduced as plausible here. In field theory, this result is more rigorously justified. ${ }^{28}$ There, the propogator is given by a time-ordered vacuum expectation value of the field operators, and derivatives that act on the time-ordering step functions are encountered when this propogator is evaluated. If these derivatives are commuted past the step functions, additional "noncovariant contact terms" develop. These terms are cancelled by noncovariant terms in the interaction Hamiltonian, which are generated during the reduction from Lagrangian form. The net result in field theory is off-mass-shell vertex functions and propogators. The M-function dynamical formalism is essentially equivalent to this field-theoretic formulation of the scattering problem. A more detailed discussion of this topic is given in Appendix D. 
This example can now be generalized to the consideration of an arbitrary Feynman diagram in terms of the $M$ fuxctions. The following rules are hypothesized:

1) At each vertex assign an off-mass-shell $\hat{M}$ function and a four-momentum conserving $\delta$ function.

2) For each intermediate state particle insert the propogator $\left(k^{2}-m^{2}+i n\right)^{-1}$.

3) Integrate over the four-momentum of each intermediate state particle.

Aside from the treatment of spin, these rules are the same as those in perturbative field theory, and it will be assumed that the formal theoretical methods of many-body field theory (e.g., Ref. 32) can be adapted to obtain a formal derivation of the M-function many-body scattering theory that has been postulated here (also, see Appendix D).

(Note: In the remainder of this dissertation, the ", notation on the operator $\hat{R}$ and the function $\hat{M}$ will not be explicitly written. Instead, the distinction betwwen $R$ and $\hat{R}$ or $M$ and $\hat{M}$ will be implied by the presence or absence of the total four-momentum conserving $\delta$ function.) 


\subsubsection{A Note on Stapp's M Function}

The above procedure can also be applied using Stapp's $M$ functions. In terms of these, the equation that is equivalent to Eq. $4-33$ is

$$
\begin{aligned}
& \overline{\mathrm{M}}\left(\mathrm{k}_{1}^{\prime} \lambda_{1}^{\prime}, \mathrm{k}_{2}^{\prime} \lambda_{2}^{\prime} ; \mathrm{k}_{1} \lambda_{1}, \mathrm{k}_{2} \lambda_{2}\right)=\frac{i}{2 \pi} \delta^{4}\left(\mathrm{k}_{1}^{\prime}+\mathrm{k}_{2}^{\prime}-\mathrm{k}_{1}-\mathrm{k}_{2}\right) \\
& \times \underset{\lambda_{3} v_{3}}{\sum} \int \mathrm{d}^{4} \mathrm{k}_{3} \delta^{4}\left(\mathrm{k}_{3}+\mathrm{k}_{1}^{\prime}-\mathrm{k}_{1}\right) \frac{1}{\mathrm{k}_{3}{ }^{2}-\mathrm{m}_{3}{ }^{2}+i \eta} \bar{H}_{b}\left(\mathrm{k}_{2}^{\prime} \lambda_{2}^{\prime} ; \mathrm{k}_{2} \lambda_{2}, \mathrm{k}_{3} \lambda_{3}\right) \\
& \times \mathrm{D}_{\lambda_{3} v_{3}}^{s_{3}}\left(\frac{\tilde{\sigma} \cdot \mathrm{k}_{3}}{\mathrm{~m}}\right) \overline{\mathrm{M}}_{\mathrm{a}}\left(\mathrm{k}_{3} v_{3}, \mathrm{k}_{1}^{\prime} \lambda_{1}^{\prime} ; \mathrm{k}_{1} \lambda_{1}\right)
\end{aligned}
$$

where $\tilde{\sigma}$ is defined in Eq. 4-14. It is seen that by defining the $M$ function as in this dissertation, the intermediate state rotation matrix has been eliminated. This rotation matrix is similar to the

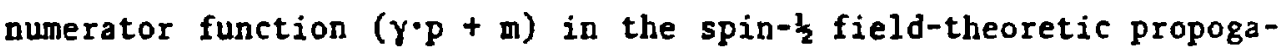
tor and, in fact, has exactly the same origin (cf., Weinberg ${ }^{28}$ and Appendix D). 


\subsection{Invariance Principles}

\subsubsection{S Matrix Invariance Principles}

In special relativistic quantum mechanics, the probability of an event occurrence does not depend on the space-time position of the event, the orientation of the event in space, or on the velocity of the event with respect to an observer. In other words, the event probability, $\mid\left\langle\left. k^{\prime} \lambda^{\prime}|s| k \lambda\right|^{2}\right.$, is assumed to be translation and Lorentz invariant.

Translation invariance is guaranteed by the fact that a translation of the scattering amplitude (Eq. 4-1) results only in a phase change. A Lorentz transformation, on the other hand, results in a more complicated expression (Eq. 4-2). Hence, the simplest way to guarantee Lorentz invariance is to assume that the scattering amplitudes $\left\langle k^{\prime} \lambda^{\prime}|S| k \lambda\right\rangle$ and $\left\langle k^{\prime} \lambda^{\prime}|S| k\right\rangle_{L}$ are equal. Equivalently, it is assumed that $U(L))^{\dagger} S U(L)=S$. This statement is called the "strong Lorentz invariance assumption".

In a similar manner, an event is space-inversion invariant (i.e., the mirror image of the event accurs with the same probability as the event) if $\mathrm{U}\left(\mathcal{d}_{\mathrm{s}}\right)^{\dagger} \mathrm{SU}\left(\ell_{\mathrm{s}}\right)=\mathrm{s}$. This invariance property is also called "P (or parity) invariance" and implies that $\left\langle k^{\prime} \lambda^{\prime}|S| k \lambda\right\rangle=\left\langle k^{\prime} \lambda^{\prime}|S| k \lambda\right\rangle_{P}$. 
An event is time-reversal invariant (or $T$ invariant) (i.e., the time-reversed image of the event occurs with the same probability as the event) if $U\left(d_{t}\right)^{\dagger} \operatorname{SU}\left(\mathcal{L}_{t}\right)=s^{\dagger}$. This invariance property is called "T invariance" and implies that $\left\langle k^{\prime} \lambda^{\prime}|S| k \lambda\right\rangle=$ $\left\langle k \lambda|S| k^{\prime} \lambda^{\prime}\right\rangle_{T}$. In these expressions, the fact that $U\left(d_{t}\right)$ is an anti-unitary operator has been used., 6

An event is charge-conjugation invariant (or $C$ invariant) if the antiparticle event probability is equal to the corresponding particle event probability (i.e., if the probability for $]_{c}+\cdots$ $+n_{c}+1_{c}^{\prime}+\cdots+n_{c}^{\prime}$ is equal to the probability for $1+\cdots+n \rightarrow$ $\left.1^{\prime}+\cdots+n^{\prime}\right)$. A statement of strong charge-conjugation invariance is made by defining an operator $U_{C}$ that maps the particle state vector $|k \lambda\rangle$ onto the antiparticle state vector $\left|(k \lambda)_{c}\right\rangle{ }^{15}$ The procedure of $\$ 2.3$ can then be used to show that $U_{C}$ is unitary and that $v_{c}|k \lambda\rangle=\eta_{c} \mid(k \lambda)_{c}>$ where $\eta_{c}$ is a phase factor. The value of $\eta_{c}$ is +1 or -1 if the particle is its own antiparticle and the particular value of $\eta_{c}$ is a new property of the single-particle state called its "charge-conjugation parity". If the particle and its antiparticle are distinct, $\eta_{c}$ can be chosen to be +1 . Strong charge-conjugation invariance assumes that $\mathrm{v}_{\mathrm{C}}^{\dagger} \mathrm{s} \mathrm{v}_{\mathrm{C}}=\mathrm{s}$.

The operations of space inversion, time reversal, and charge conjugation can be combined to define the CPT equivalent reaction as 


$$
\begin{aligned}
& \left\langle k^{\prime} \lambda^{\prime}|s| k \lambda\right\rangle_{C P T} \equiv\left(U_{C P T} \psi_{k^{\prime} \lambda^{\prime}}, s u_{C P T} \psi_{k \lambda}\right) \\
& \quad=\eta_{P^{\prime}} \eta_{c} \sum_{v^{\prime} v} D_{\lambda^{\prime} v^{\prime}}^{s^{\prime}}\left(\zeta^{\dagger}\right)\left\langle\left(k^{\prime} v^{\prime}\right)_{c}|s|(k v)_{c}>D_{v \lambda}^{s}(\zeta)\right.
\end{aligned}
$$

where $U_{C P T} \equiv U_{C} U\left(d_{s}\right) U\left(d_{t}\right)$ and $\eta_{p} \eta_{c}$ is the product of all the final state and initial state parities and charge-conjugation parities. An event is CPT invariant if $U_{\mathrm{CPT}}^{\dagger} \mathrm{S} \mathrm{U}_{\mathrm{CPT}}=\mathrm{s}^{\dagger}$, which implies $\left\langle k^{\prime} \lambda^{\prime}|S| k \lambda\right\rangle=\left\langle k \lambda|S| k^{\prime} \lambda^{\prime}\right\rangle{ }_{C P T}$. The CPT theorem, which states that all events are CPT invariant provided certain minimal restrictions are satisfied, is discussed in terms of the $M$ function in the next section.

\subsubsection{Function Invariance Principles}

Invariance principles for the on-mass-shell $M$ function follow directly from the results of $\$ 4.4 .1$ (e.g., if an event is space-inversion invariant then $M\left(k^{\prime} \lambda^{\prime} ; k \lambda\right)=M_{P}\left(k^{\prime} \lambda^{\prime} ; k \lambda\right)$ ). Two new results are established here. The first new result is the CPT theorem that was mentioned in the last section. The second is an extension of the invariance principles to off-mass-shell $M$ functions.

The CPT theorem follows directly from Eq. 4-19, which states 
that

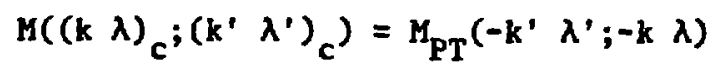

So that, if $M_{\mathrm{PT}}\left(-\mathrm{k}^{\prime} \lambda^{\prime} ;-\mathrm{k} \lambda\right)={ }_{\mathrm{PT}}\left(\mathrm{k} \lambda ; \mathrm{k}^{\prime} \lambda^{\prime}\right)$

then

$$
M\left((k \lambda)_{c} ;\left(k^{\prime} \lambda^{\prime}\right)_{c}\right)=M_{P T}\left(k \lambda ; k^{\prime} \lambda^{\prime}\right)
$$

or

$$
M\left(k^{\prime} \lambda^{\prime} ; k \lambda\right)={ }_{\mathrm{CPI}}\left(k^{\prime} \lambda^{\prime} ; k \lambda\right)
$$

This assumption (Eq. 4-37) is called the "minimal analyticity assumption" in analytic $S$ matrix theory (for a rigorous proof of the CPT theorem from this viewpoint see Stapp $^{16}$ ). The CPT theorem can also be proven within a field-theoretic framework (e.g., Streater and Wightman ${ }^{26}$ ). There, the minimal analyticity assumption is replaced by local commutativity postulates.

The minimal analyticity assumption also suggests that the off-mass-shell $M$ function is a continuation of the on-mass-shell $M$ function into unphysical regions of the energy variables. If this is assumed to be true, then the off-mass-shell $M$ function must satisfy the relation $M\left(k^{\prime} \lambda^{\prime} ; k \lambda\right)=M_{L^{\prime}}\left(k^{\prime} \lambda^{\prime} ; k \lambda\right)$ for $k^{2} \neq m^{2}$. That is, the off-mass-shell $M$ function must also be Lorentz invariant in the unphysical energy regions.

Equivalent requirements for the off-mass-shell $M$ function are also necessary to insure space-inversion and/or tine-reversal invariance for the $s$ matrix (i.e., $M\left(k^{\prime} \lambda^{\prime} ; k \lambda\right)=M_{p}\left(k^{\prime} \lambda^{\prime} ; k \lambda\right)$ 
and/or $\left.M\left(k^{\prime} \lambda^{\prime} ; k \lambda\right)=M_{T}\left(k^{\prime} \lambda^{\prime} ; k \lambda\right)\right)$; however, Eqs. 4-15 and/or 4-18 only need to be satisfied at $k^{2}=\mathrm{m}^{2}$. In other words, space-inversion and/or time-reversal invariance is guaranteed if Eqs. 4-15 and/or 4-18 are satisfied on-shell and if the continued functions $M$ and $M_{P}$ and/or $M_{T}$ are equal off-shell. $M_{P}$ and $M_{T}$ do not have to be continued to off-mass-shell values of the four-momenta via Eqs. 4-15 and 4-18. This is indeed the case in field theory where Egs. 4-15 and 4-18 are satisfied only at $k^{2}=\mathrm{m}^{2}$. (This is illustrated in the next section.) Moreover, this particular freedom in the theory can be used to define an off-mass-shell continuation of the two-body $R$ matrix that satisfies the usual parity and time-reversal properties in an angular-momentum basis (see \$5.3).

\subsection{An Example: The $\pi$ NN Vertex}

As an example of the off-mass-shell M-function formalism, consider the $\pi \mathrm{NN}$ vertex in the field-theoretic, pseudo-scalar interaction Hamiltonian model. ${ }^{24}$ In this model,

$$
H_{I}(x)=i g \bar{\psi}(x) \gamma_{5} \psi(x) \phi(x)
$$

where 8 is equal to $m_{N}$ times the $\pi N N$ coupling constant,

$$
\psi(x)=\sum_{\lambda} \int \frac{d^{3} k}{(2 \pi)^{3 / 2} 2 \varepsilon}\left\{a(k \lambda) u(k \lambda) e^{-i k \cdot x}+b(p \lambda)^{\dagger} v(p \lambda) e^{i k \cdot x}\right\}
$$




$$
\begin{aligned}
& \bar{\psi}(x)=\psi(x)^{\dagger} \gamma_{0} \\
& \phi(x)=\int \frac{d^{3} k}{(2 \pi)^{3 / 2} 2 \varepsilon}\left\{c(k) e^{-i k \cdot x}+c(k)^{\dagger} e^{i k \cdot x}\right\}
\end{aligned}
$$

$a(k \lambda)^{\dagger}$ is the creation operator for the nucleon (i.e., $a(k \lambda)^{\dagger}|0\rangle=$ |k $\lambda>$ with $\left.\left[a\left(k^{\prime} \lambda^{\prime}\right), a(k \lambda)^{\dagger}\right]=2 \varepsilon \delta^{3}\left(\vec{k}^{\prime}-\vec{k}\right) \delta_{\lambda^{\prime} \lambda^{\prime}}\right)$ and $b(k \lambda)^{\dagger}$ is the creation operator for the antinucleon (i.e., $\left.b(k \lambda)^{\dagger}|0\rangle=\left|(k \lambda)_{c}\right\rangle\right)$. $\Psi(x)$ is a four-component spinor field and $u(k \lambda)(v(k \lambda))$ is a four-component spinor that represents the nucleon (antinucleon). $\phi(x)$ is the pseudo-scalar pion field (the pion has negative parity) and isospin bas been neglected for simplicity. The energy $\varepsilon$ in the expressions for the fields is $\varepsilon \equiv\left(|\vec{k}|^{2}+m^{2}\right)^{\frac{1}{2}}$ with the appropriate mass (i.e., $m=m_{N}$ in the nucleon field and $m=m_{\pi}$ in the pion field). (Note: The factor of $m_{N}$ in Eq. 4-40 arises from the normalization of the nucleon field $-\left[\psi_{\alpha}(x), \psi_{\beta}(y)^{\dagger}\right]_{+}=$ $\left(1 / m_{N}\right) \delta_{\alpha \beta} \delta^{3}(\vec{x}-\vec{y})$ at $\left.x_{0}=y_{0}.\right)$

The four-component spinors, $u(k \lambda)$ and $v(k \lambda)$, can be represented in a diagonal $\gamma_{5}$ basis as shown in Appendix $D$.

$$
y_{5}=\left(\begin{array}{rr}
1 & 0 \\
0 & -1
\end{array}\right) \quad y_{0}=\left(\begin{array}{ll}
0 & 1 \\
1 & 0
\end{array}\right)
$$

In this basis, $u(k \lambda)=\left(\begin{array}{cc}c(k) & 0 \\ 0 & \left(c(k)^{\dagger}\right)^{-1}\end{array}\right)\left(\begin{array}{l}x_{\lambda} \\ x_{\lambda}\end{array}\right)$ 
and $\quad v(k \lambda)=\left(\begin{array}{cc}c(k) \zeta^{\dagger} & 0 \\ 0 & \left(\zeta c(k)^{\dagger}\right)^{-1}\end{array}\right)\left(\begin{array}{c}x_{\lambda} \\ -x_{\lambda}\end{array}\right)$

where $C(k)$ is the usual complementary set Lorentz transformation and $x_{\lambda}$ is a two-component spinor.

$$
x_{\frac{2}{2}}=\left(\begin{array}{l}
1 \\
0
\end{array}\right) \quad x_{-\frac{3}{2}}=\left(\begin{array}{l}
0 \\
1
\end{array}\right)
$$

The on-mass-shell vertex function for the process $N \rightarrow \pi+N$, which is shown in Fig. 4.3, can now be evaluated.

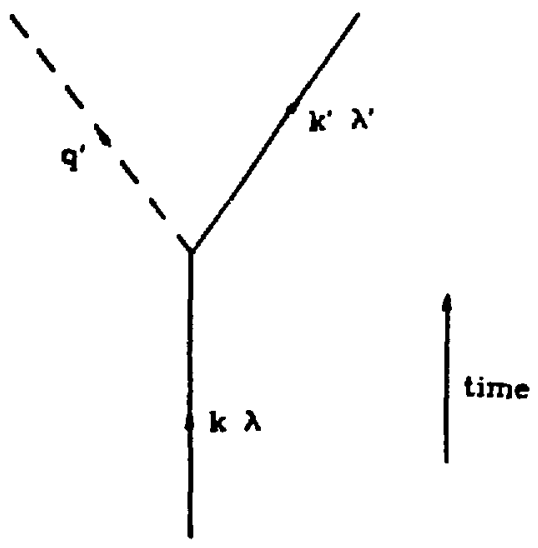

Fig. 4.3 The process $N \rightarrow \pi+N$.

The result is

$$
\begin{aligned}
& \left\langle g^{\prime} ; k^{\prime} \lambda^{\prime}\left|H_{I}(0)\right| k \lambda\right\rangle=i g(2 \pi)^{-9 / 2} \bar{u}\left(k^{\prime} \lambda^{\prime}\right) \gamma_{5} u(k \lambda) \\
& =-i g(2 \pi)^{-9 / 2} x_{\lambda}^{\dagger}\left[c\left(k^{\prime}\right)^{\dagger}\left(c(k)^{\dagger}\right)^{-1}-c\left(k^{\prime}\right)^{-1} c(k)\right] x_{\lambda}(4-48)
\end{aligned}
$$


And, the corresponding $M$ function is determined from Eqs. 4-10 and 4-25.

$$
\hat{M}\left(q^{\prime}, k^{\prime} \lambda^{\prime} ; k \lambda\right)=g(2 \pi)^{-3 / 2} \dot{\chi}_{\lambda}^{\dagger}, \frac{\sigma \cdot k^{\prime}}{m_{N}}\left(\frac{\tilde{\sigma} \cdot k}{m_{N}}-\frac{\tilde{\sigma} \cdot k^{\prime}}{m_{N}}\right) x_{\lambda} \quad(4-49)
$$

It is seen that this $\hat{M}$ function is indeed an analytic function of the four-momenta.

\subsubsection{Crossing Properties}

The on-mass-shell vertex function for the process $N+N_{c} \rightarrow \pi$, which is shown in Fig. 4.4, can also be evaluated.

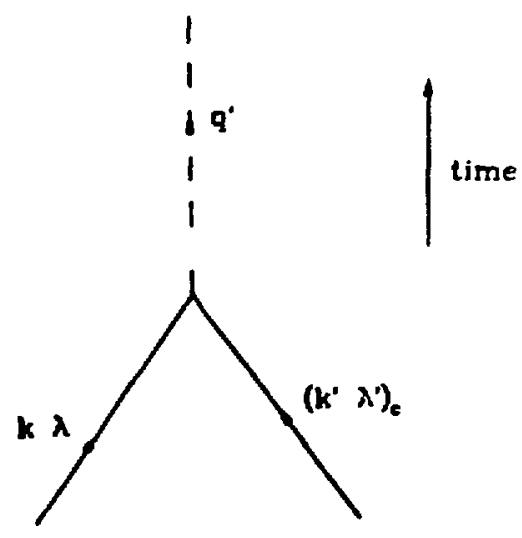

Fig. 4.4 The process $N+N_{c} \rightarrow \pi$. 
The result is

$$
\begin{aligned}
& \left\langle q^{\prime}\left|H_{I}(0)\right| k \lambda ;\left(k^{\prime} \lambda^{\prime}\right)_{c}\right\rangle=i g(2 \pi)^{-9 / 2} \bar{v}\left(k^{\prime} \lambda^{\prime}\right) \gamma_{5} v(k \lambda) \\
& =i g(2 \pi)^{-9 / 2} x_{\lambda^{\dagger}}^{\dagger} \zeta\left[c\left(k^{\prime}\right)^{\dagger}\left(c(k)^{\dagger}\right)^{-1}+c\left(k^{\prime}\right) c(k)\right] x_{\lambda} \quad(4-50)
\end{aligned}
$$

And, the corresponding $\hat{M}$ function is

$$
\hat{M}\left(q^{\prime} ; k \lambda,\left(k^{\prime} \lambda^{\prime}\right)\right)=g(2 \pi)^{-3 / 2} \chi_{\lambda^{\prime}}^{\dagger} \zeta^{-1} \frac{\sigma \cdot k^{\prime}}{m_{N}}\left(\frac{\tilde{\sigma} \cdot k}{m_{N}}+\frac{\tilde{\sigma} \cdot k^{\prime}}{m_{N}}\right) x_{\lambda}
$$

where $\zeta^{-1}=-\zeta$ has been used. Comparing this result with Eq. 4-49, it is seen that

$$
\hat{M}\left(q^{\prime} ; k^{\prime} \lambda,\left(k^{\prime} \lambda^{\prime}\right)_{c}\right)=-\sum_{v^{\prime}} D_{\lambda^{\prime} v^{\prime}}^{\frac{1}{2}}\left(\xi^{-1}\right) \hat{M}\left(q^{\prime},-k^{\prime} v^{\prime} ; k \lambda\right) \quad(4-52)
$$

This is in agreement with the crossing property, which was discussed in $\$ 4.2 .1\left(\eta_{p}=-1\right)$. 


\subsubsection{Invariance Properties}

Eq. 4-15 implies that

$$
\begin{aligned}
\hat{M}_{P^{\prime}}\left(q^{\prime}, k^{\prime} \lambda^{\prime} ; k \lambda\right) & =-g(2 \pi)^{-3 / 2} x_{\lambda^{\prime}}^{\dagger} \frac{\sigma \cdot k^{\prime}}{m_{N}} \frac{\tilde{\sigma} \cdot k^{\prime}}{m_{N}}\left(\frac{\sigma \cdot k}{m_{N}}-\frac{\sigma \cdot k^{\prime}}{m_{N}}\right) \frac{\tilde{\sigma} \cdot k}{m_{N}} x_{\lambda} \\
& =8(2 \pi)^{-3 / 2} x_{\lambda^{\prime}}^{\dagger} \frac{\sigma \cdot k^{\prime}}{m_{N}}\left(\frac{\tilde{\sigma} \cdot k}{m_{N}}-\frac{\tilde{\sigma} \cdot k^{\prime}}{m_{N}}\right) x_{\lambda} \\
& =M\left(g^{\prime}, k^{\prime} \lambda^{\prime} ; k \lambda\right)
\end{aligned}
$$

Hence, $\hat{M}\left(q^{\prime}, k^{\prime} \lambda^{\prime} ; k \lambda\right)$ is $P$ invariant. In a similar manner, it can be shown that this $M$ function is $C$ and $T$ invariant.

Now note that an essential ingredient in the evaluation of the second equality in Eq. 4-53 is

$$
\sigma \cdot k \tilde{\sigma} \cdot k=m^{2}
$$

which is only valid for mass-shell values of the four-momentum $k$. This illustrates the comment that was made at the end of \$4.4.2, where it was said that Eq. 4-15 need only be satisfied at $k^{2}=\mathrm{m}^{2}$. The third equality in Eg. 4-53 defines the off-mass-shell function $\hat{M}_{P}$ to be the same as the function $\hat{M}$. 
Chapter 5

THE TWO-BODY PROBLEM:

ON-SHELL, OFF-MASS-SHELL, AND OFF-ENERGY-SHELL

The essential aspects of off-mass-shell vertex functions and the rules for constructing a dynamical theory of interacting particles have now been established. The simplest system to which these methods can be applied consists of just two initial and two final particles. The reaction matrix for such a scattering event is studied in $\$ 5.1$, where the Hilbert space bases that were described in Chapter 3 are used to determine several of its representations. The off-mass-shell $M$ function is discussed in $\$ 5.2$, and it is shown that total-angular-momentum representations of that function can be defined - provided a real Lorentz trassformation to an effective center-of-mass reference frame exists. Such a transformation is shown to exist when the energy component of the total four-momentum is greater than the aagnitude of the total three-momentum (i.e., 
$\left.\mathrm{K}_{0}>|\overrightarrow{\mathrm{K}}|\right)$. For the isolated two-body scattering event, this requirement is always satisfied.

The dynamical two-body problem is the subject of $\$ \$ 5.4$ and 5.5. The two-body Bethe-Salpeter equation, which is a result of the Feynman rules developed in $\$ 4.3$, is described, and the Lorentz transformation to the off-mass-shell center-of-mass reference frame is defined. Within that reference frame, it is shown how to reduce the four-dimensional Bethe-Salpeter integral equation to LippmannSchwinger form, which then gives rise to a covariant definition of the off-energy-shell scattering amplitude. The net result of these sections is to relate the off-mass-shell "field-theoretic" vertex function to an off-energy-shell "potential-theoretic" vertex function. Spin and angular momentum are treated on a covariant basis throughout the discussions.

The results of $\$ \$ 5.4$ and 5.5 are applied in $\$ 5.6$ to a phenomenological study of the off-shell two-body problem. A variety of covariant methods for continuing the on-shell scattering amplitude to off-energy-shell values of the four-momenta are described, and a dispersion-theoretic equation for the determination of the offenergy-shell scattering amplitude from on-shell data is presented. 


\subsection{The R Matrix: On Shell}

The reaction amplitude was defined in $\$ 4.1$ as the expectation valne of the reaction operator, $R \equiv S-1$, between the finalsystem state vector and the initial-system state vector of a scattering event. For the two-body scattering event, these state vectors are elements of a multiple-particle Hilbert space, whose basis can be constructed in several different ways (Chapter 3). However, because the reaction amplitude is zero unless the total fourmomentum of the initial state is equal to the total four-momentum of the final state and because the reaction operator commutes with the operator $U(L)$, it is most convenient to use bases that are constructed from the two-particle fundamental state vectors (\$3.2).

This convenience is illustrated by considering the reaction amplitude when the initial (final) two-body system is represented by the state vector $\left|K\left(\vec{p} \lambda_{1} \dot{\lambda}_{2}\right)\right\rangle\left(\left|K\left(\vec{p}, \lambda_{1}^{\prime} \lambda_{2}^{\prime}\right)\right\rangle\right)$. In this representation the reaction amplitude is

$$
\begin{aligned}
\langle K(\overrightarrow{\mathrm{p}}, & \left.\left.\lambda_{1}^{\prime} \lambda_{2}^{\prime}\right)|R| K\left(\overrightarrow{\mathrm{p}} \lambda_{1} \lambda_{2}\right)\right\rangle \\
= & \left.\stackrel{0}{\mathrm{~K}}\left(\overrightarrow{\mathrm{p}}^{\prime} \lambda_{1}^{\prime} \lambda_{2}^{\prime}\right)\left|\mathrm{U}(\mathrm{C}(\mathrm{K}))^{\dagger} \mathrm{R} \mathrm{U}(\mathrm{C}(\mathrm{K}))\right| \stackrel{0}{\mathrm{~K}}\left(\overrightarrow{\mathrm{p}} \lambda_{1} \lambda_{2}\right)\right\rangle \\
= & \left.\left\langle\dot{\mathrm{K}}\left(\overrightarrow{\mathrm{p}}^{\prime} \lambda_{1}^{\prime} \lambda_{2}^{\prime}\right)|\mathrm{R}| \stackrel{\circ \mathrm{K}}{(\overrightarrow{\mathrm{p}}} \lambda_{1} \lambda_{2}\right)\right\rangle
\end{aligned}
$$


Hence, if the two-particle bases are constructed from the twoparticle fundamental state vector, the $R$ matrix in any Lorentz frame is equal to the $R$ matrix in the $C M$ reference frame. This result is certainly simpler than if the two-particle system had been represented by the direct-product of two single-particle state vectors. In that representation the relation between the $R$ natrix in an arbitrary Lorentz frame and the $R$ matrix in the $C M$ reference frame would have been needlessly complicated by rotation matrices.

\subsubsection{Total-Angular-Momentum Representations}

The $R$ watrix can be simplified further by expressing the basis of the two-particle Hilbert space in terms of total-angularmomentum basis state vectors. In these bases rotational invariance of the reaction operator implies that the $R$ matrix is diagonal in the total-angular-momentum variables $j$ and $m$ and is independent of the total-angular-momentum projection $\mathrm{m}$. This statement is proven in the Z-total-angular-momentum representation (or " $\ell$ s representation") by noting that 


$$
\begin{aligned}
& \left\langle K\left(p^{\prime} j^{\prime} m^{\prime} \ell^{\prime} s^{\prime}\right)|R| K(p j m e s)\right\rangle \\
& =\left\langle K\left(p^{\prime} j^{\prime} m^{\prime} \ell^{\prime} s^{\prime}\right)\left|U(A)^{\dagger} R U(A)\right| K(p j m \& s)\right\rangle \\
& =\sum_{\mu^{\prime} \mu} D_{m^{\prime} \mu^{\prime}}^{j^{\prime}}\left(A^{\dagger}\right)\left\langle\grave{K}\left(p^{\prime} j^{\prime} \mu^{\prime} \ell^{\prime} s^{\prime}\right)|R| K(p \quad j m \ell s)\right\rangle D_{\mu m}^{j}(A)
\end{aligned}
$$

when $A$ is any rotation. Hence, because the rotation matrices are irreducible representations of the rotation group, the $\mathbf{R}$ matrix, when considered as a $\left(2 j^{\prime}+1\right) \times(2 j+1)$ matrix with the row (column) index $m^{\prime}(m)$, must be square and proportional to the identity. (This is a statement of Schurr's lemma, which was briefly discussed in $\$ 1.4$. )

$$
\left\langle K\left(p^{\prime} j^{\prime} m^{\prime} \ell^{\prime} s^{\prime}\right)|R| K(p j m \ell s)\right\rangle=\delta_{j}{ }^{\prime} j \delta_{m^{\prime} m} R^{j}\left(w ; \ell^{\prime} s^{\prime} ; \ell s\right)
$$

where $w=\varepsilon_{1},\left(p^{\prime}\right)+\varepsilon_{2},\left(p^{\prime}\right)=\varepsilon_{1}(p)+\varepsilon_{2}(p)$. An equivalent result is obtained in the helicity-total-angular-nomentum representation.

$$
\begin{gathered}
\left\langle K\left(p^{\prime} j^{\prime} m^{\prime} \lambda_{1}^{\prime} \lambda_{2}^{\prime}\right)|R| K\left(p \text { jm } \lambda_{1} \lambda_{2}\right)\right\rangle \\
=\delta_{j^{\prime} j} \delta_{m^{\prime} m} R^{j}\left(w ; \lambda_{1} \lambda_{2}^{\prime} ; \lambda_{1} \lambda_{2}\right)
\end{gathered}
$$




\subsubsection{Space-Inversion Invariance}

If the reaction operator is space-inversion invariant (i.e., $\left.U\left(f_{s}\right)^{\dagger} R U\left(f_{s}\right)=R\right)$, Eqs. 3-37 and 3-38 imply that

$$
R^{j}\left(w ; \ell^{\prime} s^{\prime} ; \ell s\right)=\eta_{p}(-1)^{\ell^{\prime}+l} R^{j}\left(w ; \ell^{\prime} s^{\prime} ; \ell s\right)
$$

and

$$
R^{j}\left(w ; \lambda_{1}^{\prime} \lambda_{2}^{\prime} ; \lambda_{1} \lambda_{2}\right)=\eta_{p}(-1)^{j-s_{1}^{j}-s_{2}^{\prime}}(-1)^{j-s_{1}-s_{2}} R^{j}\left(\omega ;-\lambda_{1}^{\prime}-\lambda_{2}^{\prime} ;-\lambda_{1}-\lambda_{2}\right)
$$

where $\eta_{p}=\eta_{1}^{\prime} \eta_{2}^{\prime} \eta_{1} \eta_{2}$ and $\eta_{i}\left(\eta_{i}^{\prime}\right)$ is the parity of the $i$ th initial (final) state particle.

These results typify the problems that are associated with using the helicity representation in a problem that has a spaceinversion invariant scattering operator. In the $\ell s$ representation, space-inversion invariance implies that the $R$ matrix is zero unless $\eta_{p}(-1)^{l^{\prime}+l}=+1$; whereas, in the helicity representation the $R$ matrix has the property shown in Eq. 5-6. In either case, spaceinversion invariance results in a reduction in the number of independent reaction amplitudes, but only in the $l s$ representation does this reduction result in $R$ matrix elements that are zero. Of course, parity eigenstates in the helicity representation can be defined (e.8., Eq. 3-39), but this requires restriction on the 
values of the helicities that are needed to span the two-particle Hilbert space. Thus, for a space-inversion invariant scattering operator, the $2 s$ representation appears to be a more computationally efficient representation.

It should also be remembered, however, that working in the is representation often requires the use of Clebsch-Gordan coefficients (e.g., Eq. 3-25). This aspect of the $\mathrm{ls}$ representation introduces complexity that is not present in the helicity representation. Thus, there is a trade-off in complexity. The $\ell$ s representation has simple properties when the problem is space-inversion invariant, and the helicity representation is simple with respect to angular-momentum algebra. These and other distinctions between these two representations will become more apparent in later sections of this chapter and in Chapter 6.

The philosophy of this author is that the helicity representation allows for more concise theoretical manipulations; whereas, the $\ell s$ representation should be used for computations. But, fluency in moving between the two representations is necessary to the development of simple approaches to the problem of spin in the relativistic many-body problem. In the remainder of this dissertation the helicity representation will be used almost exclusively for the derivation of equations. Results in the $\ell s$ representation will be derived therefrom. 


\subsubsection{Time-Reversal Invariance}

If the reaction operator is time-reversal invariant (i.e., $\left.U\left(f_{t}\right)^{\dagger} R U\left(d_{t}\right)=R^{\dagger}\right)$, Eqs. 3-41 and 3-42 imply that

$$
R^{j}\left(w ; \ell^{\prime} s^{\prime} ; \ell s\right)=R^{j}\left(w ; \ell s ; \ell^{\prime} s^{\prime}\right)
$$

and $R^{j}\left(w ; \lambda_{1} \lambda_{2}^{\prime} ; \lambda_{1} \lambda_{2}\right)=R^{j}\left(w ; \lambda_{1} \lambda_{2} ; \lambda_{1}^{\prime} \lambda_{2}^{\prime}\right)$

\subsection{The Center-of-Mass M Function: Off-Mass-Shell}

In $\$ 5.1$, many useful results concerning the angular-momentum representations of the reaction amplitude were derived. These results were based on the properties of the total-angular-momentum bases of the two-particle Hilbert space and on the properties of the reaction operator. In this section, similar results will be derived for the off-mass-shell $M$ function; but, because the $M$ function is not the expectation value of an operator, these results must be derived by appealing to the properties of that function, which were established in Chapter 4, rather than to the properties of the two-particle state vectors.

$A$ necessary input to the angular-momentum expansion of the $R$ atrix was the ability to ake a Lorentz transformation from an arbitrary Lorentz frame to the CM reference frame. Within that 
reference frame, the relative three-momentun was defined and the total-angular-momentum was defined by expanding the $R$ natrix, considered as a function of the angles of these relative momenta, in terms of the complete set of rotation matrices. An equivalent procedure for the $M$ function, which is based on its Lorentz transformation properties, is also possible - provided there exists a real Lorentz transformation that when applied to the total fourmomentum results in a four-momentum whose three-vector components are zero. In other words, a "CM M function" can be defined if there exists a real Lorentz transformation $c(K)$ such that $c(K)^{-1} K=\stackrel{0}{K}$ with $\stackrel{0}{K}=(\underset{\sim}{w}, \overrightarrow{0}) . \quad(K$ is the off-mass-shell total four-momentum and $\underset{\sim}{\omega}$ must be real and greater than zero.) This Lorentz transformation exists if $K_{0}>|\vec{K}|$. (Note: This requirement was not recognized by Namyslowski, ${ }^{4}$ who applied a similar method to the spin-0 two-body problem.)

For a physical two-body scattering event, this restriction is satisfied at all steps in the perturbative expansion of the $M$ function. However, if the off-mass-shell $\mathrm{H}$ function is to be included as a component in a many-body process, there will be values of $K_{0}$ that are less than $|\vec{K}|$. In this case, a suitable generalization of the methods developed here must be considered. This aspect of the $M$ function has not been thoroughly investigated. In the remainder of this chapter, only $M$ functions that have total four-momentum $K$ with $K_{0}>|\vec{K}|$ are considered. 
The $C M \quad M$ function is defined by first relabeling the $M$ function in terms of the total four-momentum

$$
K=K_{1}^{\prime}+K_{2}^{\prime}=K_{1}+K_{2}
$$

and the $W-G$ relative four-momentum ( $\left.E_{q} .3-9\right)$

$$
\Delta=\frac{1}{2}\left(K_{1}-K_{2}\right)-\frac{1}{2 s}\left(m_{1}^{2}-m_{2}^{2}\right) K
$$

where $s \equiv K^{2}={\underset{\sim}{w}}^{2}$; so that,

$$
M\left(K_{1}^{\prime} \lambda_{1}^{\prime}, K_{2}^{\prime} \lambda_{2}^{\prime} ; K_{1} \lambda_{1}, K_{2} \lambda_{2}\right) \equiv M\left(K ; \Delta^{\prime} \lambda_{1}^{\prime} \lambda_{2}^{\prime} ; \Delta \lambda_{1} \lambda_{2}\right)
$$

Then, within the region $K_{0}>|\vec{k}|$, the Lorentz transformation $c(K)$ is defined by

$$
c(K)^{-1} K=\stackrel{\circ}{K}: \stackrel{\circ}{K} \equiv(\underset{\sim}{w}, \overrightarrow{0})
$$

where $\underset{\sim}{w}$ is real and greater than zero. This Lorentz transformation can be applied to the $M$ function (Eq. 4-10) to obtain

$$
\begin{aligned}
M\left(K ; \Delta^{\prime} \lambda_{1}^{\prime} \lambda_{2}^{\prime} ; \Delta \lambda_{1} \lambda_{2}\right) & =\sum_{v^{\prime} v} D_{\lambda_{1}^{\prime} v_{1}^{\prime}}^{s_{1}^{\prime}}(C(K)) D_{\lambda_{2}^{\prime} v_{2}^{\prime}}^{s_{2}^{\prime}}(C(K)) \\
& \times M\left(K_{i} P^{\prime} v_{1}^{\prime} v_{2}^{\prime} ; p v_{1} v_{2}\right) D_{v_{1} \lambda_{1}}^{s_{1}}\left(C(K)^{-1}\right) D_{v_{2} \lambda_{2}}^{s_{2}}\left(C(K)^{-1}\right)
\end{aligned}
$$

where $p^{\prime} \equiv c(K)^{-1} \Delta^{\prime}, p \equiv c(K)^{-1} \Delta$, and the sum over $v^{\prime}$ and $v$ implies 
a summation over $v_{1}^{\prime}, v_{2}^{\prime}$ and $v_{1}, v_{2}$. (This notation will be used throughout this chapter.) The four-momentum $p$ will be called the "relative four-momentum" of the two-body system, and it should be noted that $p$ is defined with respect to the off-mass-shell fourmomenta $K_{1}$ and $K_{2}$ and not with respect to the three-momenta, $\vec{K}_{1}$ and $\vec{K}_{2}$, and the on-shell energies, $\varepsilon_{1}\left(\left|\vec{K}_{1}\right|\right)$ and $\varepsilon_{2}\left(\left|\vec{K}_{2}\right|\right)$. If the relative momentum had been defined with respect to these on-shell fourmomenta, a unique Lorentz transformation to the CM "reference frame" could not have been defined. This problem is often encountered in medium-energy physics ${ }^{10}$ and is overcome by the formalism that is being presented in this dissertation.

The function $M\left(\AA^{\prime} ; p^{\prime} \lambda_{1}^{\prime} \lambda_{2}^{\prime} ; p \quad \lambda_{1} \lambda_{2}\right)$ will be called the "CM $M$ function", and Eq. 5-13 shows that this $M$ function is dependent on the particular choice of the complementary set of Lorentz transformations, $C(K)$. (Note: This dependence, which introduces kinematic singularities if $K_{0}<|\vec{K}|$, is precisely the reason for considering only $M$ functions that have a total four-momentum $K$ with $\left.K_{0}>|\vec{K}|.\right)$ The $C M$ function in the helicity representation is defined by the choice

$$
c(K)=R(\hat{K}) Z\left(x_{K}\right)
$$

with $\cosh \chi_{K}=\gamma_{K}, \quad \sinh x_{K}=\gamma_{K}\left|\vec{\beta}_{K}\right|$ 


$$
\vec{\beta}_{\mathrm{K}}=\frac{\overrightarrow{\mathrm{K}}}{\mathrm{K}_{0}} \text { and } \gamma_{\mathrm{K}}=\frac{\mathrm{K}_{0}}{\left(\mathrm{~K}_{0}{ }^{2}-\overrightarrow{\mathrm{K}}^{2}\right)^{\frac{1}{2}}}
$$

The CM $M$ function in the $Z$ representation is defined by the choice $C(K)=L\left(x_{K}, \hat{K}\right)$.

\subsubsection{Total-Angular-Momentum Representations}

A total-angular-momentum representation of the CM M function is obtained by considering it as a function of the relative energies, $p_{0}^{\prime}$ and $p_{0}$, the magnitudes of the relative three-momenta, $\left|\vec{p}^{\prime}\right|$ and $|\vec{p}|$, and the polar angles of the three-momenta, $\hat{p}^{\prime}$ and $\hat{p}$. The results of $\$ 1.4 .1$ then allow the CM $M$ function to be expanded in the complete set of rotation matrices.

The CM M function in the "helicity-total-angular-momentum representation" (or, simply, the "belicity representation") is defined as 


$$
\begin{aligned}
& M\left(K_{i} ; p_{0}^{\prime}\left|\vec{p}^{\prime}\right| j^{\prime} m^{\prime} \lambda_{1}^{\prime} \lambda_{2}^{\prime} ; p_{0}|\vec{p}| \lambda_{1} \lambda_{2}\right) \\
& =\left(\frac{2 j^{\prime}+1}{4 \pi}\right)^{\frac{1}{2}}\left(\frac{2 j+1}{4 \pi}\right)^{\frac{1}{2}} e^{i \pi s_{2}^{i}-i \pi s_{2}} \\
& \times \int d^{2} \hat{p}^{\prime} d^{2} \hat{p} D_{m^{\prime}, \lambda_{1}^{\prime}-\lambda_{2}^{\prime}}^{j^{\prime}}\left(R\left(\hat{p}^{\prime}\right)\right) D_{m, \lambda_{1}-\lambda_{2}}^{j}(R(\hat{p}))^{*} \\
& \times \sum_{v^{\prime} v} D_{\lambda_{1}^{\prime} v_{1}^{\prime}}^{s_{1}^{\prime}}\left(R\left(\hat{p}^{\prime}\right)^{-1}\right) D_{\lambda_{2}^{\prime} v_{2}^{\prime}}^{s_{2}^{\prime}}\left(\left(R\left(\hat{p}^{\prime}\right) R(\pi, \pi)\right)^{-1}\right) \\
& \times \quad M\left(K_{i}^{\prime} ; p^{\prime} v_{1}^{\prime} v_{2}^{\prime} ; \mathrm{P} \quad v_{1} v_{2}\right) \mathrm{D}_{v_{1} \lambda_{1}}^{s_{1}}(\mathrm{R}(\hat{\mathrm{p}})) \mathrm{D}_{v_{2} \lambda_{2}}^{\mathrm{s}_{2}}(\mathrm{R}(\hat{\mathrm{p}}) \mathrm{R}(\pi, \pi))
\end{aligned}
$$

(Note: To determine the inverse of this expression see Eqs. 1-27 through 1-30.) This definition is suggested by the results of the total-angular-momentum analysis of the two-particle helicity state vectors, but this definition does not introduce any new kinematic singularities. The spin-projection quantum numbers in this representation are rotational invariants.

The CM M function in the "ls-total-angular-momentum representation" (or, simply, the "ls representation") is defined as in Eq. 3-32. 


$$
\begin{aligned}
& M\left(K_{i} p_{0}^{\prime}\left|\vec{p}^{\prime}\right| j^{\prime} m^{\prime} \ell^{\prime} s^{\prime} ; p_{O}|\vec{p}| j \mid l s\right)=\left(\frac{2 \ell^{\prime}+1}{2 j^{\prime}+1}\right)^{\frac{1}{2}}\left(\frac{2 \ell+1}{2 j+1}\right)^{\frac{1}{2}}
\end{aligned}
$$

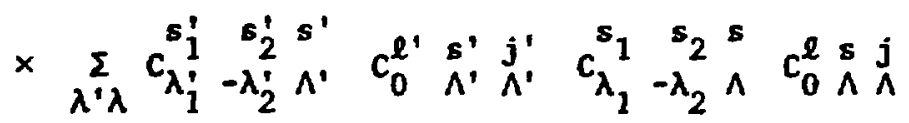

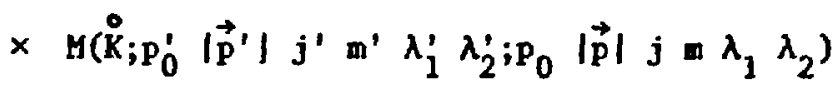

where $\Lambda^{\prime} \equiv \lambda_{1}^{\prime}-\lambda_{2}^{\prime}$ and $\Lambda \equiv \lambda_{1}-\lambda_{2}$.

These definitions and the Lorentz transformation property of the $M$ function imply that

$$
\begin{aligned}
& M\left(\stackrel{\circ}{K} ; F_{0}^{\prime}\left|\vec{p}^{\prime}\right| j^{\prime} m^{\prime} \lambda_{j}^{\prime} \lambda_{2}^{\prime} ; p_{0}|\vec{p}| j m \lambda_{1} \lambda_{2}\right)=\sum_{\mu^{\prime} \mu} D_{m}^{j^{\prime} \mu^{\prime}}(R) \\
& \times \quad M\left(\stackrel{\circ}{K} ; p_{0}^{\prime}\left|\vec{p}^{\prime}\right| j^{\prime} \mu^{\prime} \lambda_{j}^{\prime} \lambda_{2}^{\prime} ; p_{0}|\vec{p}| j \mu \lambda_{1} \lambda_{2}\right) D_{\mu m}^{j}(R)
\end{aligned}
$$

when $R$ is an arbitrary rotation. Hence, by Schurr's lemma (cf., Eq. 5-2), this $M$ function must be proportional to $\delta_{j{ }^{\prime} j} \delta_{\mathrm{m}^{+} m}$ and independent of $\mathrm{m}$.

i.e., $M\left(\stackrel{\circ}{*} ; p_{0}^{\prime}\left|\vec{p}^{\prime}\right| j^{\prime}\right.$ ' $\lambda_{1}^{\prime} \lambda_{2}^{\prime} ; p_{0}|\vec{p}| j$ m $\left.\lambda_{1} \lambda_{2}\right)$

$$
=\delta_{j^{\prime} j} \delta_{m^{\prime} m} M^{j}\left(w_{j} p_{0}^{\prime}\left|\vec{p} \prime^{\prime}\right| \lambda_{1}^{\prime} \lambda_{2}^{\prime} ; p_{0}|\vec{p}| \lambda_{1} \lambda_{2}\right)
$$

A similar result holds for the $M$ function in the $l s$ representation. 


\subsubsection{Space-Inversion Invariance}

In $\$ 4.2$, it was shown that the space-inversion transformation gives rise to a new on-mass-shell M function,

$$
\begin{aligned}
& M_{\mathrm{P}}\left(\mathrm{K}_{1}^{\prime} \lambda_{1}^{\prime}, \mathrm{K}_{2}^{\prime} \lambda_{2}^{\prime} ; \mathrm{K}_{1} \lambda_{1}, \mathrm{~K}_{2} \lambda_{2}\right)=\eta_{\mathrm{p}} \underset{v^{\prime} v}{\sum} \mathrm{D}_{\lambda_{1}^{\prime} v_{1}^{\prime}}^{s_{1}^{\prime}}\left(\frac{\sigma \cdot \mathrm{K}_{1}^{\prime}}{\mathrm{m}_{1}^{\prime}}\right) \mathrm{D}_{\lambda_{2}^{\prime} v_{2}^{\prime}}^{s_{2}^{\prime}}\left(\frac{\sigma \cdot \mathrm{K}_{2}^{\prime}}{\mathrm{m}_{2}^{\prime}}\right) \\
& \times M\left(\mathrm{I}_{\mathrm{s}} \mathrm{K}_{1}^{\prime} v_{1}^{\prime}, \mathrm{I}_{\mathrm{s}} \mathrm{K}_{2}^{\prime} v_{2}^{\prime} ; \mathrm{I}_{\mathrm{s}} \mathrm{K}_{1} v_{1}, \mathrm{I}_{\mathrm{s}} \mathrm{K}_{2} v_{2}\right) \mathrm{D}_{v_{1} \lambda_{1}}^{s_{1}}\left(\frac{\tilde{\sigma} \cdot \mathrm{K}_{1}}{\mathrm{~m}_{1}}\right) \mathrm{D}_{v_{2} \lambda_{2}}^{s_{2}}\left(\frac{\tilde{\sigma} \cdot \mathrm{K}_{2}}{\mathrm{~m}_{2}}\right)
\end{aligned}
$$

where $\tilde{\sigma} \equiv\left(\sigma_{0},-\vec{\sigma}\right)$ and $\eta_{p}=\eta_{1}^{\prime} \eta_{2}^{\prime} \eta_{1} \eta_{2}$ (cf., Eq. 4-15). In the helicity-total-angular-momentum representation, this $M$ function is (use $\left.R\left(I_{s} \hat{k}\right)=R(\hat{k}) R(\pi, \pi) R(\xi, \hat{z})\right)$

$$
\begin{aligned}
& M_{\mathrm{P}}^{j}\left(\underset{\sim}{u_{j}} ;\left|\overrightarrow{p^{\prime}}\right| \lambda_{1}^{\prime} \lambda_{2}^{\prime} ;|\vec{p}| \lambda_{1} \lambda_{2}\right) \\
& =\eta_{p} e^{\lambda_{1}^{\prime} x_{1}^{\prime}+\lambda_{2}^{\prime} x_{2}^{\prime}-\lambda_{1} x_{1}-\lambda_{2} x_{2}}(-1)^{j-s_{1}^{\prime}-s_{2}^{\prime}} \\
& \times(-1)^{j-s_{1}-s_{2}} M^{j}\left(\underset{\sim}{w} ;\left|\vec{p}^{\prime}\right|-\lambda_{1}^{\prime}-\lambda_{2}^{\prime} ;|\vec{p}|-\lambda_{1}-\lambda_{2}\right)
\end{aligned}
$$

where the on-mass-shell values of the four-momenta are given by (see §3.1) 


$$
\begin{aligned}
& p_{0}^{\prime}=p_{0}=0 \quad \underset{w}{w}=w^{\prime}=w \\
& w^{\prime} \equiv w_{1}^{\prime}+w_{2}^{\prime} \quad: \quad w_{i}^{\prime} \equiv \varepsilon_{i},(|\vec{p} \cdot|)=\left(|\vec{p} \cdot|^{2}+m_{i}^{2}\right)^{\frac{3}{2}} \\
& w \equiv w_{1}+w_{2} \quad: \quad w_{i} \equiv \varepsilon_{i}(|\vec{p}|)=\left(|\vec{p}|^{2}+m_{i}{ }^{2}\right)^{\frac{3}{2}} \\
& \tanh x_{i}^{\prime} \equiv \frac{\left|\vec{p}^{\prime}\right|}{w_{i}^{\prime}} \quad \tanh x_{i} \equiv \frac{|\vec{p}|}{w_{i}}
\end{aligned}
$$

and the factors $e^{\lambda X}$ arise from the $2 s+1$ dimensional representation of the Lorentz group ( $\$ 1.4)$.

e.g., $D_{\lambda \nu}^{s}\left(\frac{1}{a_{i}}\left(w_{i} \sigma_{0}-|\vec{p}| \sigma_{3}\right)\right)=D_{\lambda \nu}^{s}\left(\cosh x_{i} \sigma_{0}-\sinh x_{i} \sigma_{3}\right)=\delta_{\lambda \nu} e^{\lambda x_{i}}$

The $M$ function labels $p_{0}$ and $p_{0}^{\prime}$ have been suppressed in Eq. 5-21 because they are equal to zero.

The function $M_{P}^{j}$ can now be continued off-mass-shell by recalling (\$4.4) that a space-inversion invariant scattering operator only requires $M=M_{P}$ as given in Eq. 5-20 at on-mass-shell values of the four-momenta. This result allows one to define the off-mass-shell continuation of $\mathrm{M}_{\mathrm{P}}^{\mathrm{j}}$ as 


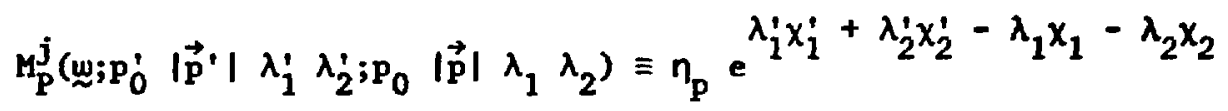

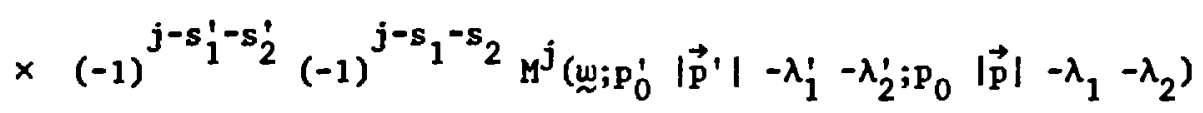

where $x_{i}^{\prime}$ and $x_{i}$ are still given by Eq. 5-24 (i.e., defined in terms of $\left|\vec{p}^{\prime}\right|$ and $\left.|\vec{p}|\right)$. Space-inversion invariance is guaranteed if $M^{j}=$ $\mathrm{M}_{\mathrm{P}}^{\mathrm{j}}$ at all values of the four-momenta.

\subsubsection{Time-Reversal Invariance}

If the scattering operator is time-reversal invariant, the on-shell $M$ function satisfies the relation (see Eg. 4-15 and \$4.4)

$$
\begin{aligned}
& H\left(K_{1}^{\prime} \lambda_{1}^{\prime}, K_{2}^{\prime} \lambda_{2}^{\prime} ; K_{1} \lambda_{1}, K_{2} \lambda_{2}\right)=M\left(K_{1} \lambda_{1}, K_{2} \lambda_{2} ; K_{1}^{\prime} \lambda_{1}^{\prime}, K_{2}^{\prime} \lambda_{2}^{\prime}\right) \\
& =\sum_{v^{\prime} v} D_{\lambda_{1} v_{1}}^{s_{1}}\left(\zeta^{-1} \frac{\sigma \cdot K_{1}}{m_{1}}\right) D_{\lambda_{2} v_{2}}^{s_{2}}\left(\zeta^{-1} \frac{\sigma \cdot K_{2}}{m_{2}}\right) \\
& \times H\left(I_{s} K_{1} \lambda_{1}, I_{s} K_{2} \lambda_{2} ; I_{s} K_{1}^{\prime} \lambda_{1}^{\prime}, I_{s} K_{2}^{\prime} \lambda_{2}^{\prime}\right) D_{v_{1}^{\prime} \lambda_{1}^{\prime}}^{s_{1}^{\prime}}\left(\frac{\tilde{\sigma} \cdot K_{1}}{m_{1}^{\prime} \zeta}\right) D_{v_{2}^{\prime} \lambda_{2}^{\prime}}^{s_{2}^{\prime}}\left(\frac{\tilde{\sigma} \cdot K_{2}}{m_{2}^{\prime} \zeta}\right)
\end{aligned}
$$


This implies that

$$
\begin{aligned}
& M^{j}\left(w_{w} ; p_{0}^{\prime}|\vec{p} \cdot| \lambda_{1}^{\prime} \lambda_{2}^{\prime} ; p_{0}|\vec{p}| \lambda_{1} \lambda_{2}\right)
\end{aligned}
$$

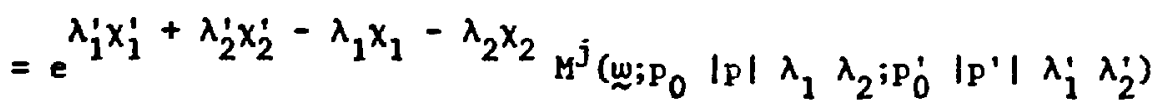

$$
\begin{aligned}
& \text { (5-28) }
\end{aligned}
$$

5.3 The T Function: Off-Mass-She11

Equations 5-26 and 5-28 suggest that a new off-mass-shell function be defined as

$$
\begin{aligned}
& \mathrm{T}^{\mathrm{j}}\left(\underset{\sim}{\omega} ; \mathrm{P}_{0}^{\prime}\left|\overrightarrow{\mathrm{p}}^{\prime}\right| \lambda_{1}^{\prime} \lambda_{2}^{\prime} ; \mathrm{P}_{0}|\overrightarrow{\mathrm{p}}| \lambda_{1} \lambda_{2}\right)
\end{aligned}
$$

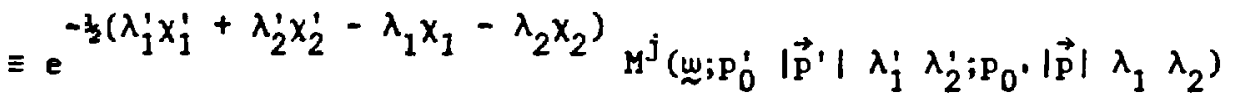

which has the space-inversion property

$$
\begin{aligned}
& \mathrm{T}_{\mathrm{P}}^{j}\left(\underset{\sim}{w} ; \mathrm{p}_{0}^{\prime}\left|\overrightarrow{\mathrm{p}}^{\prime}\right| \lambda_{1}^{\prime} \lambda_{2}^{\prime} ; \mathrm{p}_{0}|\overrightarrow{\mathrm{p}}| \lambda_{1} \lambda_{2}\right) \\
& =\eta_{p}(-1)^{j-s_{1}^{j}-s_{2}^{\prime}}(-1)^{j-s_{1}-s_{2}} T^{j}\left(w_{w} ; p_{0}^{\prime}\left|\vec{p}^{\prime}\right|-\lambda_{1}^{j}-\lambda_{2}^{\prime} ; p_{0}|\vec{p}|-\lambda_{1}-\lambda_{2}\right)
\end{aligned}
$$

(space-inversion invariance implies that $T^{j}=T_{P}^{j}$ ) and is time-

127 
reversal invariant if

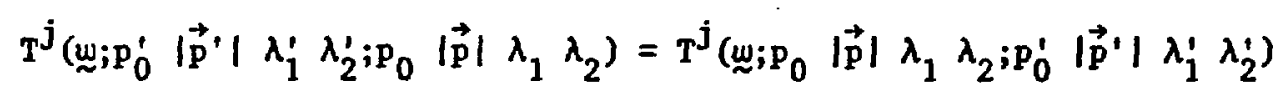

These results are reminiscent of the $R$ matrix (Eqs. 5-6 and 5-8). In fact,

$$
D_{\lambda \nu}^{s}(z(x))=\delta_{\lambda \nu} e^{\lambda(x / 2)}
$$

(cf., Eq. 1-41) and Eq. 5-16 imply that

$$
\begin{aligned}
\mathrm{T}\left(\stackrel{\circ}{\mathrm{K}} ; \mathrm{p}^{\prime} \quad \lambda_{1}^{\prime} \lambda_{2}^{\prime} ; \mathrm{P} \lambda_{1} \lambda_{2}\right)=\sum_{v^{\prime} v} \mathrm{D}_{\lambda_{1}^{\prime} v_{1}^{\prime}}^{\mathrm{s}_{1}^{\prime}}\left(\mathrm{C}\left(\overline{\mathrm{k}}_{1}^{\prime}\right)^{-1}\right) \mathrm{D}_{\lambda_{2}^{\prime} v_{2}^{\prime}}^{\mathrm{s}_{2}^{\prime}}\left(\mathrm{C}\left(\overline{\mathrm{k}}_{2}^{\prime}\right)^{-1}\right) \\
\quad \times M\left(\stackrel{\circ}{\mathrm{K}} ; \mathrm{p}^{\prime} \quad v_{1}^{\prime} v_{2}^{\prime} ; \mathrm{p} v_{1} v_{2}\right) \mathrm{D}_{v_{1} \lambda_{1}}^{\mathrm{s}_{1}}\left(\mathrm{C}\left(\overline{\mathrm{k}}_{1}\right)\right) \mathrm{D}_{v_{2} \lambda_{2}}^{\mathrm{s}_{2}}\left(\mathrm{C}\left(\overline{\mathrm{k}}_{2}\right)\right)
\end{aligned}
$$

where $\bar{k}_{i}^{\prime}=\left(\varepsilon_{i},(|\vec{p}|),, \pm \vec{p}^{\prime}\right)$ and $\bar{k}_{i}=\left(\varepsilon_{i}(|\vec{p}|), \pm \vec{p}\right)\left({ }^{\prime}+\right.$ if $i=1,{ }^{\prime}$ if $i=2)$. Hence, the reaction amplitude (Eq. 4-9) is equal to $-2 \pi i$ times the on-mass-shel1 $T$ function; so that, Eq. 5-33 defines the off-mass-shell continuation of the reaction amplitude.

This definition is based on the definition of the CM $M$ function (Eq. 5-13), which implies that Eq. 5-33 can not be applied in an arbitrary Lorentz frame. 


$$
\begin{aligned}
& \text { i.e., } T\left(K_{1}^{\prime} \lambda_{1}^{\prime}, K_{2}^{\prime} \lambda_{2}^{\prime} ; K_{1} \lambda_{1}, K_{2} \lambda_{2}\right) \neq \sum_{v^{\prime} v} D_{\lambda_{1}^{\prime} v_{1}^{\prime}}^{s}\left(C\left(\bar{K}_{1}^{\prime}\right)^{-1}\right) D_{\lambda_{2}^{\prime} v_{2}^{\prime}}^{s}\left(C\left(\bar{R}_{2}^{\prime}\right)^{-1}\right) \\
& \quad \times M\left(K_{1}^{\prime} v_{1}^{\prime}, K_{2}^{\prime} v_{2}^{\prime} ; K_{1} v_{1}, K_{2} v_{2}\right) D_{v_{1} \lambda_{1}}^{s}\left(C\left(\bar{K}_{1}\right)\right) D_{v_{2} \lambda_{2}}^{s_{2}}\left(C\left(\bar{R}_{2}\right)\right)
\end{aligned}
$$

An equality is not allowed here because this is not a Lorentz invariant equation. The key to a correct off-mass-shell continuation is in defining that continuation with respect to the Lorentz invariants $|\vec{p}| \mid$ and $|\vec{p}|$.

In the $\ell$ sepresentation, the $T$ function is defined as in Eq. 5-17; and, if parity is conserved,

$$
\begin{aligned}
& T^{j}\left(\underset{\sim}{w} ; p_{0}^{\prime}\left|\vec{p}^{\prime}\right| \ell^{\prime} s^{\prime} ; p_{0}|\vec{p}| \& s\right) \\
& =\eta_{p}(-1)^{\ell^{\prime}+\ell} T^{j}\left(w_{\sim} ; p_{0}^{\prime}\left|\vec{p}^{\prime}\right| \ell^{\prime} s^{\prime} ; p_{0}|\vec{p}| \ell s\right)
\end{aligned}
$$

This result is far simpler than the corresponding $M$ function equation. For this reason and because the $T$ function is more intimately connected with the reaction amplitude, most of the results tha: appear in subsequent sections of this dissertation, which are derived in terms of the $M$ function, are rewritten in terms of the $T$ function. 


\subsection{The Two-Body Bethe-Salpeter Equation}

A dynamic equation that describes the scattering event $1+2+1+2$ can be written for the two-body $M$ function by appealing to the Feynman rules (Chapter 4). The resulting equation, which is essentially equivalent to the field-theoretic Bethe-Salpeter equation, $24,25,33$ is

$$
\begin{aligned}
M\left(K_{1}^{\prime} \lambda_{1}^{\prime}, K_{2}^{\prime} \lambda_{2}^{\prime} ; K_{1} \lambda_{1}, K_{2} \lambda_{2}\right)=I\left(K_{1}^{\prime} \lambda_{1}^{\prime}, K_{2}^{\prime} \lambda_{2}^{\prime} ; K_{1} \lambda_{1}, K_{2} \lambda_{2}\right) \\
+\sum_{\lambda^{\prime}} \int d^{4} K_{1}^{\prime \prime} d^{4} K_{2}^{\prime \prime} I\left(K_{1}^{\prime} \lambda_{1}^{\prime}, K_{2}^{\prime} \lambda_{2}^{\prime} ; K_{1}^{\prime \prime} \lambda_{1}^{\prime \prime}, K_{2}^{\prime \prime} \lambda_{2}^{\prime \prime}\right) \\
\quad \times \frac{1}{K_{1}^{\prime \prime 2}-m_{1}{ }^{2}+i \eta} \frac{1}{K_{2}^{\prime}{ }^{2}-m_{2}{ }^{2}+i \eta} M\left(K_{1}^{\prime} \lambda_{1}^{\prime}, K_{2}^{\prime} \lambda_{2}^{\prime \prime} ; K_{1} \lambda_{1}, K_{2} \lambda_{2}\right) \\
\quad \times \delta^{4}\left(K_{1}^{\prime \prime}+K_{2}^{\prime \prime}-K_{1}-K_{2}\right)
\end{aligned}
$$

where $K_{1}^{\prime}+K_{2}^{\prime}=K_{1}+K_{2}, I\left(K_{1}^{\prime} \lambda_{1}^{\prime}, K_{2}^{\prime} \lambda_{2}^{\prime} ; K_{1} \lambda_{1}, K_{2} \lambda_{2}\right)$ is the sum of all two-body irreducible Feynman diagrams (i.e., the sum of all diagrams that cannot be split into two pieces by cutting just lines that represent particles 1 and 2 as intermediate states), and a four-momentum conserving $\delta$ function has been factored out of $I$ within the integral.

The Bethe-Salpeter equation for the CH $M$ function is obtained by relabeling the functions $I$ and $M$ in terms of the total fourmomentum and the appropriate W-G relative four-monentum (Egs. 5-9 and 5-10); so that, if the integration variables are changed, 
$\left(K_{1}^{\prime}, K_{2}^{\prime}\right)+\left(K^{\prime}, \Delta^{\prime \prime}\right)$, and the definition of the CM $M$ function is applied within the region $k_{0}>|\vec{k}|$, the following equation results:

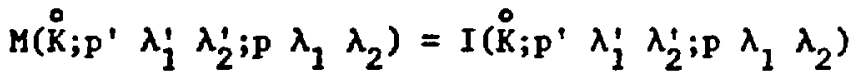

$$
\begin{aligned}
& +\sum_{\lambda^{\prime}} \int d^{4} p^{\prime \prime} I\left(\AA_{i p}^{\prime} \lambda_{1}^{\prime} \lambda_{2}^{\prime} ; p^{\prime \prime} \lambda_{1}^{\prime} \lambda_{2}^{\prime \prime}\right) \\
& \times g_{1}\left(k_{1}^{\prime \prime 2}\right) g_{2}\left(k_{2}^{\prime \prime}{ }^{2}\right) M\left(K_{;} ; p^{\prime \prime} \lambda_{1}^{\prime \prime} \lambda_{2}^{\prime \prime} ; p \lambda_{1} \lambda_{2}\right)
\end{aligned}
$$

where $g_{i}\left(k^{2}\right)=\left(k^{2}-m_{i}{ }^{2}+i \eta\right)^{-1}, k_{i}=c(k)^{-1} k_{i}$, and the other notation is explained in $\$ 5.2$.

In the helicity-total-angular-momentum representation, the Bethe-Salpeter equation is

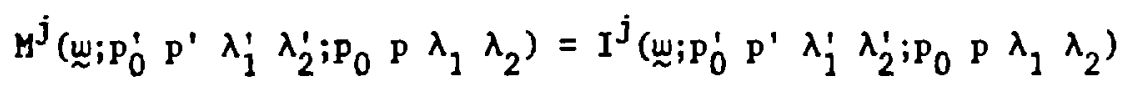

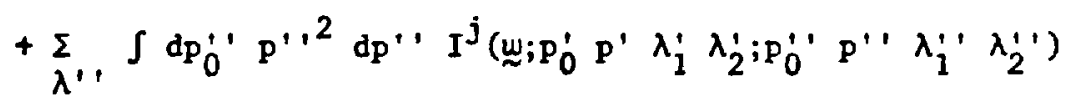

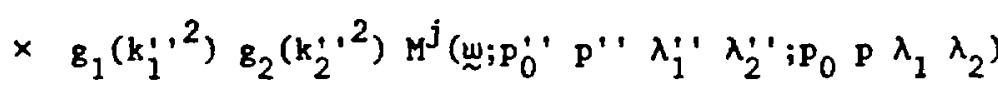

where the notation $p \equiv|\vec{p}|$ is used here and will be used in the remainder of this dissertation when there can be no confusion with the four-momentum $p$. 


\subsection{Reduction of the Two-Body Bethe-Salpeter Equation to Lippmann- Schwinger Form}

As written in \$5.4, the Bethe-Salpeter equation is a fourdimensional integral equation for the CM $M$ function (Eq. 5-37); or, after total-angular-momentum reduction, it is a set of coupled two-dimensional integral equations for the reduced $M$ function (Eq. 5-38). As such, the Bethe-Salpeter equation is difficult to solve. Another problem with the Bethe-Salpeter equation is that the "potential", I, is an unknown function in most cases of interest. It would be convenient if this potential could be parameterized as in nonrelativistic scattering theory or if the Bethe-Salpeter equation could be used to derive methods for the covariant determination of an off-mass-shell $M$ function from on-shell scattering data. The means of obtaining these desired goals, as originally proposed by Blankenbecler and Sugar $4,53,54$ for the spin 0 system, is to replace the propogator in the Bethe-Salpeter equation, $G\left(\mathrm{k}_{1}{ }^{2}, \mathrm{k}_{2}{ }^{2}\right)$ $\equiv \mathrm{g}_{1}\left(\mathrm{k}_{1}{ }^{2}\right) \mathrm{g}_{2}\left(\mathrm{k}_{2}{ }^{2}\right)$, with a new propogator such that a three-dimensional integral equation for the CM $M$ function results. That is,

let $G \equiv G_{0}+\left(G-G_{0}\right) \equiv G_{0}+G_{1}$ where $G \equiv 8_{1} 8_{2}$

so that $M=B+B G_{0} M$

with $B=I+I G_{1} B$ 
and choose $G_{0}$ such that Eq. 5-40 is a three-dimensional integral equation for $M$ and $\mathrm{Eq}$. 5-41 is a four-dimensional integral equation for $B$. This method does not alter the basic Bethe-Salpeter equation (i.e., the complete off-mass-shell $\mathrm{CH} M$ function is still determined), but it does transfer the complexity to the determination of a new potential term. Eq. 5-40 will be shown to have a form that is similar to the nonrelativistic Lippmann-Schwinger equation and will be used in $\$ 5.5 .2$ to define an "off-energy-shell" reaction amplitude. This amplitude and its corresponding potential can be treated by the usual methods of nonrelativistic scattering theory. In $\$ 5.6$, covariant phenomenological methods for the determination of the off-energy-shell reaction amplitude will be discussed.

\subsubsection{Unitarity}

Any theory of the two-body reaction $1+2 \rightarrow 1+2$ must result in a unitary $S$ matrix (Chapter 4). A propogator $G_{0}$ can be chosen such that at least part of this requirement (two-body unitarity with respect to particles 1 and 2 ) is manifestly satisfied by the $M$ function that is calculated from $E_{q} \cdot 5-40$, and this choice implies that any phenomenological theory that is derived from Eq. 5-40 will also satisfy this "minimal" requirement. The on-shell unitarity relations are presented in this section. 
The unitarity relation for the $S$ matrix can be written as

$$
\left\langle f\left|S^{\dagger} S\right| i\right\rangle=\sum_{n}\left\langle f\left|S^{\dagger}\right| n\right\rangle\langle n|S| i\rangle=\sum_{n}\langle n|S| f\rangle^{*}\langle n|S| i\rangle=\langle f \mid i\rangle
$$

where $|f\rangle(|i\rangle)$ represents the final (initial) system state vector and $\Sigma|n\rangle\langle n|$ represents a sum over a complete set of basis state vectors for the multiple-particle Hilbert space (i.e., a sum over all permissible one-particle, two-particle, $\cdots$ states). In terms of the reaction operator, $R \equiv S-1$, the unitarity relation is

$$
R+R^{\dagger}=R_{R}^{\dagger}=R^{\dagger} R
$$

so that,

$\langle f|R| i\rangle+\langle i|R| f\rangle^{*}=\sum_{n}\langle f|R| n\rangle\langle i|R| n\rangle^{*}=\sum_{n}\langle n|R| f\rangle^{*}\langle n|R| i\rangle(5-44)$

Hence, the unitarity relation for the two-body $M$ function is

$$
\begin{aligned}
M\left(K_{1}^{\prime} \lambda_{1}^{\prime}, K_{2}^{\prime}\right. & \left.\lambda_{2}^{\prime} ; K_{1} \lambda_{1}, K_{2} \lambda_{2}\right)-M\left(K_{1}^{\prime} \lambda_{1}^{\prime}, K_{2}^{\prime} \lambda_{2}^{\prime} ; K_{1} \lambda_{1} K_{2} \lambda_{2}\right)^{\dagger} \\
= & -2 \pi i \sum_{\lambda^{\prime}} \int \frac{d^{3} K_{1}^{\prime}}{2 \varepsilon_{1}^{\prime \prime}} \frac{d^{3} K_{2}^{\prime}}{2 \varepsilon_{2}^{\prime \prime}} M\left(K_{1}^{\prime} \lambda_{1}^{\prime}, K_{2}^{\prime} \lambda_{2}^{\prime} ; K_{1}^{\prime} \lambda_{1}^{\prime}, K_{2}^{\prime \prime} \lambda_{2}^{\prime \prime}\right) \\
& \times M\left(K_{1}^{\prime} \lambda_{1}^{\prime \prime}, K_{2}^{\prime \prime} \lambda_{2}^{\prime \prime} ; K_{1} \lambda_{1}, K_{2} \lambda_{2}\right)^{\dagger} \delta^{4}\left(K_{1}^{\prime}+K_{2}^{\prime}-K_{1}-K_{2}\right)+\cdots
\end{aligned}
$$

where $K_{1}^{\prime}+K_{2}^{\prime}=K_{1}+K_{2}$, the dots represent all parts of the unitarity relation except the two-body part, which is explicitly shown, 
and

$$
\begin{aligned}
& \sum_{v^{\prime}} D_{\lambda_{1}^{\prime} v_{1}^{\prime}}^{s_{1}^{\prime}}\left(\frac{\sigma \cdot K_{1}^{\prime}}{m_{1}^{\prime}}\right) D_{\lambda_{2}^{\prime} v_{2}^{\prime}}^{s_{2}^{\prime}}\left(\frac{\sigma \cdot K_{2}^{\prime}}{m_{2}^{\prime}}\right) M\left(K_{1}^{\prime} v_{1}^{\prime}, K_{2}^{\prime} v_{2}^{\prime} ; K_{1} \lambda_{1}, K_{2} \lambda_{2}\right)^{\dagger} \\
& \quad \equiv \sum_{v} M\left(K_{1} v_{1}, K_{2} v_{2} ; K_{1}^{\prime} \lambda_{1}^{\prime}, K_{2}^{\prime} \lambda_{2}^{\prime}\right)^{*} D_{v_{1} \lambda_{1}}^{s_{1}}\left(\frac{\sigma \cdot K_{1}}{m_{1}}\right) D_{v_{2} \lambda_{2}}^{s_{2}}\left(\frac{\sigma \cdot K_{2}}{m_{2}}\right)(5-46)
\end{aligned}
$$

A total four-momentum conserving $\delta$ function has also been factored out of one of the $M$ functions, and it should be remembered that this relation involves only on-mass-shell functions (i.e., $k_{i}=\left(\varepsilon_{i}, \vec{k}_{i}\right)$ with $\left.\varepsilon_{i} \equiv\left(\left|\vec{K}_{i}\right|^{2}+m_{j}^{2}\right)^{\frac{3}{2}}\right)$.

The $M$ function unitarity relation can also be written as

$$
\begin{aligned}
& M\left(K_{1}^{\prime} \lambda_{1}^{\prime}, K_{2}^{\prime} \lambda_{2}^{\prime} ; K_{1} \lambda_{1}, K_{2} \lambda_{2}\right)-M\left(K_{1}^{\prime} \lambda_{1}^{\prime}, K_{2}^{\prime} \lambda_{2}^{\prime} ; K_{1} \lambda_{1}, K_{2} \lambda_{2}\right)^{\dagger} \\
& =-2 \pi i \sum_{\lambda^{\prime}} f d^{4} K_{1}^{\prime} d^{4} K_{2}^{\prime} \delta^{+}\left(K_{1}^{\prime}{ }^{2}-m_{1}^{2}\right) \delta^{+}\left(K_{2}^{\prime}{ }^{2}-m_{2}^{2}\right) \\
& \quad \times M\left(K_{1}^{\prime} \lambda_{1}^{\prime}, K_{2}^{\prime} \lambda_{2}^{\prime} ; K_{1}^{\prime} \lambda_{1}^{\prime}, K_{2}^{\prime} \lambda_{2}^{\prime}\right) M\left(K_{1}^{\prime} \lambda_{1}^{\prime}, K_{2}^{\prime \prime} \lambda_{2}^{\prime \prime} ; K_{1} \lambda_{1}, K_{2} \lambda_{2}\right)^{\dagger} \\
& \quad \times \delta^{4}\left(K_{1}^{\prime}+K_{2}^{\prime}-K_{1}-K_{2}\right)+\cdots
\end{aligned}
$$

and the integration variables can be changed, $\left(K_{1}^{\prime}, K_{2}^{\prime \prime}\right) \rightarrow\left(K^{\prime \prime}\right.$, $\left.\Delta^{\prime \prime}\right)(\mathrm{cf} ., \mathrm{Eq} .3-10)$. The resulting equation can be expressed in terms of the CM $M$ function. 


$$
\begin{aligned}
& M\left(\stackrel{\circ}{K} ; P^{\prime} \lambda_{1}^{\prime} \lambda_{2}^{\prime} ; P \lambda_{1} \lambda_{2}\right)-M\left(\stackrel{\circ}{K} ; P^{\prime} \lambda_{1}^{\prime} \lambda_{2}^{\prime} ; P \lambda_{1} \lambda_{2}\right)^{\dagger} \\
& =-2 \pi i \sum_{\lambda^{\prime}} \int d^{4} p^{\prime \prime} \frac{w^{\prime \prime}}{2 w_{1}^{\prime \prime} w_{2}^{\prime \prime}} \delta^{+}\left(w^{\prime \prime 2}-{\underset{w}{2}}^{2}\right) \delta\left(p_{0}^{\prime \prime}\right) \\
& M\left(\stackrel{\circ}{K} ; p^{\prime} \lambda_{1}^{\prime} \lambda_{2}^{\prime} ; p^{\prime \prime} \lambda_{1}^{\prime} \lambda_{2}^{\prime \prime}\right) M\left(\stackrel{\circ}{K} ; p^{\prime \prime} \lambda_{1}^{\prime} \lambda_{2}^{\prime \prime} ; \mathrm{p} \lambda_{1} \lambda_{2}\right)^{\dagger}+\cdots
\end{aligned}
$$

where $p_{0}^{\prime}=p_{0}=0, \underset{w}{w}=w^{\prime}=w(\stackrel{\circ}{K} \equiv(\underset{\sim}{w}, \overrightarrow{0}))$, and the other notation is explained in \$3.1. In the helicity-total-angular-momentum representation,

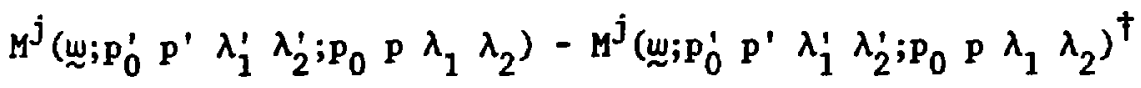

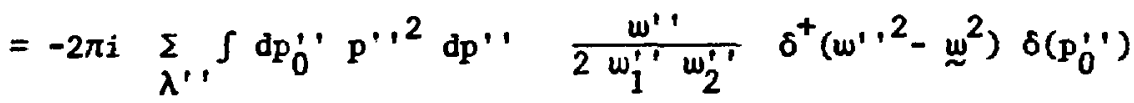

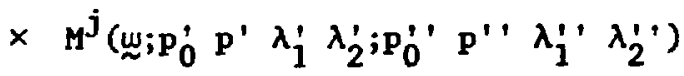

$$
\begin{aligned}
& \times \mathrm{M}^{\mathrm{j}}\left(\underset{w}{w} ; \mathrm{p}_{0}^{\prime \prime} \mathrm{p}^{\prime \prime} \lambda_{1}^{\prime \prime} \lambda_{2}^{\prime \prime} ; \mathrm{p}_{0} \mathrm{p} \lambda_{1} \lambda_{2}\right)^{\dagger}
\end{aligned}
$$

where $p \equiv|\vec{p}|$ in this equation. 


\subsubsection{The Off-Energy-Shell Vertex Functions}

It was shown in Eqs. 5-39 through 5-41 that the BetheSalpeter equation could be written as

$$
M=B+B G_{0} M=B+M G_{0} B
$$

and it was suggested that an appropriate choice of the propogator, $G_{0}$, would result in this being a three-dimensional integral equation for M. In \$5.5.1, it was suggested that the unitarity relation could be used to specify this propogator.

These suggestions are fulfilled by nultiplying Eq. 5-50 on the right with $\left(1+G_{0}^{*} M^{\dagger}\right)$, multiplying the relation

$$
M^{\dagger}=B^{\dagger}+B^{\dagger} G_{O}^{*} M^{\dagger}
$$

on the left with $\left(1+M G_{0}\right)$, and subtracting. The result is

$$
M\left(1+G_{0}^{*} M^{\dagger}\right)-\left(1+M G_{0}\right) M^{\dagger}=\left(1+M G_{0}\right)\left(B-B^{\dagger}\right)\left(1+G_{0}^{*} M^{\dagger}\right)
$$

This implies that

$$
M-M^{\dagger}=M\left(G_{0}-G_{0}^{*}\right) M^{\dagger}+\left(1+M G_{0}\right)\left(B-B^{\dagger}\right)\left(1+G_{0}^{*} M^{\dagger}\right)
$$


Thus, if

$$
\begin{aligned}
& G_{0}\left(k_{1}^{\prime \prime 2}, k_{2}^{\prime \prime 2}\right)-G_{0}\left(k_{1}^{\prime}{ }^{2}, k_{2}^{\prime \prime 2}\right)^{\star} \\
& =-2 \pi i \frac{w^{\prime \prime}}{2 w_{1}^{\prime \prime} w_{2}^{\prime \prime}} \delta^{+}\left(w^{\prime}{ }^{2}-w^{2}\right) \delta\left(p_{0}^{\prime \prime}\right)
\end{aligned}
$$

and if the term with $B-B^{\dagger}$ is equal to the parts of Eq. 5-48 that are indicated by dots, then the $M$ function, which is determined from Eq. 5-50, yields an $S$ matrix that satisfies the unitarity requirement.

A propogator that has the property shown in Eq. $5-54$ is

$$
\begin{aligned}
& G_{0}\left(k_{1}^{\prime}{ }^{2}, k_{2}^{+2}\right) \equiv G_{0}\left(w^{2}, w^{+2}, p_{0}^{\prime \prime 2}, w_{p}^{\prime \prime}\right) \\
& =\frac{i}{2 \pi} \int d x \frac{G_{0}\left(x, w^{\prime \prime 2}, p_{0}^{\prime \prime 2}, w_{0}\right)-G_{0}\left(x, w^{+1}, p_{0}^{\prime \prime 2}, w_{0}^{\prime \prime}\right)}{x-w^{\prime}{ }^{2}+i \eta} \\
& =\frac{w^{\prime \prime}}{2 w_{1}^{\prime \prime} w_{2}^{\prime \prime}} \frac{1}{w^{2}-w^{\prime}{ }^{2}+i \eta} \delta\left(p_{0}^{\prime \prime}\right)
\end{aligned}
$$

A proof of this result is obtained using

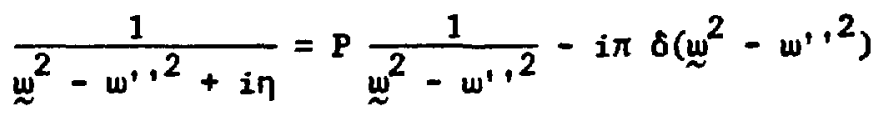

where $P \frac{1}{x}$ denotes the principle value distribution function of $\frac{1}{x}$. It should also be remembered that $\underset{\sim}{w}$ was defined to be greater than zero. 
With this definition of $G_{0}$, the Bethe-Salpeter equation for the $C M M$ function in the helicity-total-angular-momentum representation at $p_{0}^{\prime}=p_{0}=0$ is

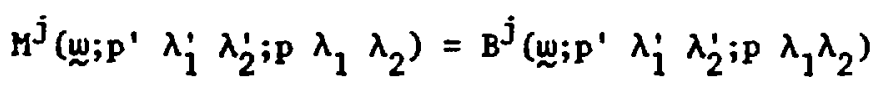

$$
\begin{aligned}
& +\sum_{\lambda}, \int p^{\prime \prime 2} \mathrm{dp}^{\prime \prime} \frac{\omega^{\prime \prime}}{2 \omega_{1}^{\prime \prime} \omega_{2}^{\prime \prime}} \frac{1}{\underline{w}^{2}-w^{\prime}{ }^{2}+i \eta}
\end{aligned}
$$

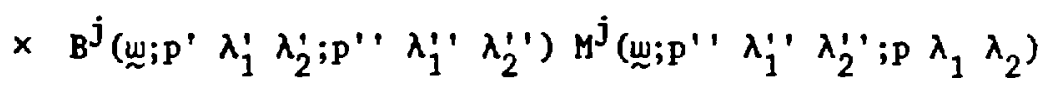

where $p \equiv|\vec{p}|$ and the relative-energy variables (e.g., $p_{0}$ ), which are equal to zero, have been suppressed. Furthermore, because $p_{0}^{\prime \prime}=0$, Eqs. 5-9 and 5-10 imply that

$$
\left(k_{1}^{\prime \prime}\right)_{0}=\frac{1}{2 \underset{\sim}{w}}\left({\underset{\sim}{w}}^{2}+m_{1}^{2}-m_{2}^{2}\right)
$$

and

$$
\left(k_{2}^{\prime}\right)_{0}=\frac{1}{2 w}\left(\underset{w}{2}-m_{1}^{2}+m_{2}^{2}\right)
$$

Equation 5-57 has the same form as the off-energy-shell LippmannSchwinger equation.

The off-energy-shell CM M function is now defined to be the off-mass-shell CM M function evaluated at zero relative energy. An equation that is identical in form to Eq. 5-57 is satisfied by the off-energy-shell $\mathrm{T}$ function. 
(Note: Other propogators that satisfy Eq. 5-54 can, of course, be defined; but, the propogator that is given in Eq. 5-55 is the only one that reduces the Bethe-Salpeter equation to a threedimensional integral equation in terms of the relative threemomentum of the two-body system. As such, there is no distinction made between particle 1 and 2 in the reduction proceedure -- e.g., one particle is not put on its mass-shell while the other is left off its mass-shell. The method of reduction that has been demonstrated here is similar to that used by Namyslowski in Refs. 4 and 54.)

\subsection{Covariant Phenomenology}

In addition to providing a Lippmann-Schwinger type equation for the off-energy-shell scattering amplitude, the reduction procedure of $\$ 5.5$ provides valuable insight into the structure of the vertex function itself. In this section, the two-body off-energyshell $T$ function is considered and phenomenological models of that function are discussed. The basic methods are introduced in $\$ \$ 5.6 .1$ and 5.6.2 through a consideration of the spin 0 system. In \$5.6.1, a variety of methods for covariantly continuing the on-shell $T$ function to off-energy-shell values of the four-mowenta are presented. In \$5.6.2, a dispersion theoretic equation that relates the off-energy-shell $T$ function to on-shell scattering data is derived, 
and the properties of the $T$ function at threshold (i.e., $|\vec{p}| \rightarrow 0$ ) are established. These results are then generalized to include particles with spin in 55.6 .3 . One of the most interesting results of this analysis is model independent technique (i.e., based only on the $M$ function formalism) of relating different off-energy-shell and off-mass-shell continuation methods.

\subsubsection{Covariant Off-Energy-Shell Continuation: Spin 0}

If the off-mass-shell $T$ function is considered as a function of the Lorentz invariants that can be formed from the four-momenta of the particles involved in the scattering event, a covariant method for continuing that function from on-shell values of the four-momenta into unphysical (i.e., off-energy-shell or off-massshell) regions of phase space can be developed. The procedure by which this is accomplished begins with a consideration of $T\left(K_{i} ; p^{\prime} ; p\right)$, which is a function of the polar angles of $\vec{p}$, and $\vec{p}$ and of the following Lorentz invariants:

$$
\begin{aligned}
& \underset{\sim}{w}=\left(k^{2}\right)^{\frac{1}{2}} \quad: \underset{\sim}{w}>0 \text { and real } \\
& p_{0}^{\prime} \text { and } p_{0} \quad: p_{0}^{\prime}=\frac{K \cdot \Delta^{\prime}}{\underset{w}{w}} \quad p_{0}=\frac{K \cdot \Delta}{\underset{w}{w}}
\end{aligned}
$$

and $|\vec{p} \cdot|$ and $|\vec{p}|:|\vec{p}|=\left(p_{0}^{2}-\Delta^{2}\right)^{\frac{1}{2}} \quad|\vec{p} \cdot|=\left(p_{0}^{\prime 2}-\Delta^{\prime 2}\right)^{\frac{1}{2}}(5-62)$ 
where the total four-momentum, $K$, and the $W-G$ relative four momenta, $\Delta^{\prime}$ and $\Delta$, are defined in Eqs. 5-9 and 5-10. The polar angles of $\vec{p}$, and $\vec{p}$ are not Lorentz invariants, but the $T$ function can be manipulated to yield a Lorentz invariant argument that will replace these angular variables. This is achieved by writing the rotation $R\left(\hat{p}^{\prime}\right)$ as

$$
R\left(\hat{p}^{\prime}\right) \equiv R(\hat{p}) R\left(\alpha, \theta_{p^{\prime} p}, \beta\right)
$$

where $\theta_{p} p$ is the angle between the three-vectors $\vec{p}$, and $\vec{p}$, the angles $\alpha$ and $\beta$ are the Euler angles required to make Eq. 5-63 an identity, and $R\left(\alpha, \theta_{p^{\prime}}, \beta\right) \equiv R(\alpha, \hat{z}) R\left(\theta_{p^{\prime} p}, \hat{y}\right) R(\beta, \hat{z})$. This identity and the rotation property of the $T$ function then imply that

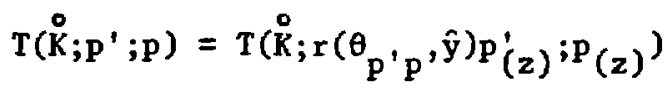

where $p_{(z)}^{\prime}=\left(p_{0}^{\prime}, 0,0,\left|\vec{p}^{\prime}\right|\right)$ and $p_{(z)}=\left(p_{0}, 0,0,|\vec{p}|\right)$. In terms of Lorentz invariants, the angle $\theta_{p^{\prime} p}$ is given by

$$
\Delta^{\prime} \cdot \Delta=\mathbf{p}_{0}^{\prime} \mathbf{P}_{0}-\left|\overrightarrow{p^{\prime}}\right||\vec{p}| \cos \theta_{p^{\prime} p}
$$

Thus, the $T$ function can be expressed as

$$
T\left(\stackrel{\circ}{K} ; r\left(\theta_{p^{\prime} p}, \hat{y}\right) p_{(z)}^{\prime} ; P_{(z)}\right) \equiv T\left(K^{2}, \Delta^{\prime 2}, \Delta^{2}, K \cdot \Delta^{\prime}, K \cdot \Delta, \Delta^{\prime} \cdot \Delta\right)
$$

with $T\left(\stackrel{\circ}{K} ; p^{\prime} ; p\right)$ given by Eq. 5-64. (Note: Since the $M$ function for the spin 0 system is a Lorentz scalar, the result that is shown in 
Eq. 5-66 could have been obtained by noting that the only Lorentz scalars that can be derived from the four-momenta $K, \Delta^{\prime}$, and $\Delta$ are $K^{2}, \Delta^{\prime 2}, \Delta^{2}, K \cdot \Delta^{\prime}, K \cdot \Delta$, and $\Delta^{\prime} \cdot \Delta$. However, this simple method cannot be used for a system with spin.)

Eq. 5-66 slows that the off-mass-shell $T$ function is a function of six independent Lorentz invariant variables. The offenergy-shell $T$ function is obtained by setting $P_{0}^{\prime}$ and $P_{0}$ (or, equivalently, $K \cdot \Delta^{\prime}$ and $K \cdot \Delta$ ) equal to zero and is a function of four Lorentz invariant variables. The on-shell $T$ function, which is obtained by setting $w^{9}=w=\underset{\sim}{w}$, is dependent on just two variables, $\underset{w}{ }$ and $\cos \theta_{p^{\prime}} p^{.}$This suggests that the simplest method of covariantly continuing the on-shell $T$ function to off-energy-shell values of the four-momenta is to assume that the on-shell function is the same as the off-energy-shell function when expressed in terms of an appropriate pair of Lorentz invariant variables.

For example, suppose that the on-shell function $T\left(\underset{\sim}{w}, \cos \theta_{p^{\prime}} p^{\prime}\right)$ is known, and suppose that the off-energy-shell $T$ function is only dependent on the variables $x^{2}$ and $\Delta^{\prime} \cdot \Delta$. This implies that the off-energy-shell $T$ function way be obtained by replacing the variable $\cos \theta_{p^{\prime} p}$ in the on-shell function with the variable $\left(\Delta^{\prime} \cdot \Delta / \mathbb{R}^{2}\right)$, where (Eq. 3-7)

$$
R=\frac{1}{2 w} \xi\left(w^{2}, m_{1}^{2}, m_{2}^{2}\right)
$$


(Note: For simplicity, it has been assumed that the initial and final masses are the same - $m_{1}^{i}=m_{1}$ and $m_{2}^{\prime}=m_{2}$ ). In other words, the off-energy-shell continuation is obained by the rep itcement

$$
{ }^{\cos \theta_{p^{\prime} p}}+\frac{p^{\prime} p}{\mathrm{R}^{2}} \cos \theta_{p^{\prime} p}
$$

in the on-shell $\mathrm{T}$ function. This method will be called the "fixed $\Delta^{\prime} \cdot \Delta "$ off-energy-shell model.

Other procedures can, of course, be adopted. The procedure that is most commonly used in analytic $S$ matrix theory, which will be called the "fixed $t$ " model, is to assume that the off-energyshell $T$ function is only dependent on $K^{2}$ and $\left(\Delta^{\prime}-\Delta\right)^{2}\left(t \equiv\left(\Delta^{\prime}-\Delta\right)^{2}\right)$. This assumption implies that the replacement

$$
\cos \theta_{p^{\prime} p}+1-\frac{1}{2 p^{2}}\left(p^{2}+p^{2}-2 p^{\prime} p \cos \theta_{p^{\prime} p^{\prime}}\right)
$$

should be made in the on-shell $\mathrm{T}$ function.

These examples illustrate that the basic phenomenological method for relativistic scattering theory is based on a reduction in the number of Lorentz invariant variables on which the off-massshell vertex function depends. Moreover, that reduction is obtained by restricting the four-momenta to off-energy-shell values and by assuming a restricted dependence on the remaining variables. 
To continue with the discussion of this phenomenological method, it is most instructive to consider the off-energy-shell $T$ function in the total-angular-momentum representation. In that representation, the $T$ function is (cf., Eqs. 5-16 and 5-63)

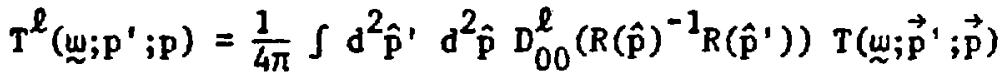

$$
\begin{aligned}
& =2 \pi \int_{-1}^{1} d\left(\cos \theta_{p^{\prime} p^{\prime}}\right) d_{00^{l}}^{\ell}\left(\cos \theta_{p^{\prime} p^{\prime}}\right) T\left(\underset{\sim}{w}, p^{\prime}, p, \cos \theta_{p^{\prime} p^{\prime}}\right)(5-70)
\end{aligned}
$$

where $p_{0}^{\prime}=p_{0}=0$ (these labels have been suppressed), $p^{\prime} \equiv\left|\vec{p}^{\prime}\right|$,

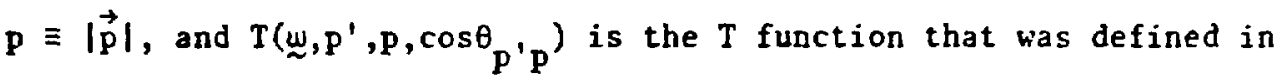
Eq. 5-64. The argument of the $d$ matrix has also been changed to

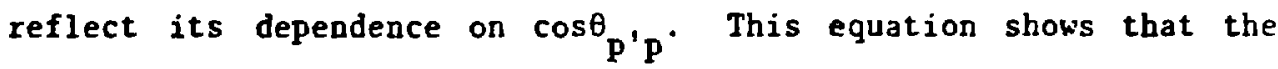
total-angular-momentum expansion of the $T$ function is defined with respect to the already continued off-energy-shell function. Thus, if the off-energy-shell $T$ function is given in terms of the on-shell function by the replacement shown in Eq. 5-68, then

$$
\mathrm{T}^{\ell}\left(\underset{\sim}{w}, \mathrm{p}^{\prime}, \mathrm{p}\right)=2 \pi \frac{\mathrm{p}^{2}}{\mathrm{p}^{\prime} \mathrm{p}} \int_{-\mathrm{p}^{\prime} \mathrm{p} / \mathrm{p}^{2}}^{\mathrm{p}^{\prime} \mathrm{p} / \mathrm{p}^{2}} \mathrm{dx} \mathrm{d}_{00^{2}}^{\ell}\left(\frac{\mathrm{p}^{2}}{\mathrm{p}^{\prime} \mathrm{p}} \mathrm{x}\right) \mathrm{T}(\underset{\sim}{\underset{w}{w}, x})
$$

where $T(\underset{\sim}{w}, x)$ is the on-shell $T$ function and $x$ corresponds to $\cos \theta_{p} p$ in that function. 
Eq. 5-71 now shows that the on-shell function, $T(\underset{w}{w} x)$, must be known for values of $x$ that are outside of the range $-1 \leq x \leq 1$. (Note: This does not contradict the angular-momentum expansion, which is shown in Eq. 5-70, because that expansion is defined with respect to the angle $\theta_{p^{\prime} p}$ in the already continued off-energy-shell $T$ function and the cosine of this angle always satisfies the requi rement $-1 \leqq \cos \theta_{p^{\prime} p} \leqq 1$.)

As an example of this result, suppose that the on-shell $T$ function can be expressed as

$$
T(\underset{\sim}{w}, \cos \theta)=\sum_{\ell}\left(\frac{2 \ell+1}{4 \pi}\right) d_{00}^{\ell}(\cos \theta) T^{\ell}(\underset{\sim}{\omega})
$$

where $-1 \leqq \cos \theta \leqq 1$, and suppose that the expansion coefficients, $\mathrm{T}^{\ell}\left(\underset{w}{)}\right.$, are negligible for $\ell$ greater than $\ell_{\max }$. This equation is now assumed to be the correct functional form of the off-energy-shell $T$ function. The continuation procedure then requires $\cos \theta$ to be replaced by $\left(p^{\prime} p \cos \theta_{p^{\prime}} p^{\prime} p^{2}\right)$. This implies that

$$
\begin{aligned}
& T\left(\underset{\sim}{w}, p^{\prime}, p, \cos \theta_{p^{\prime} p^{\prime}}\right)=\sum_{\ell=0}^{\ell}\left(\frac{2 \ell+1}{4 \pi}\right) d_{00}^{\ell}\left(\frac{p^{\prime} p}{R^{2}} \cos \theta_{p^{\prime} p}\right) T^{\ell}(\underset{w}{)})
\end{aligned}
$$

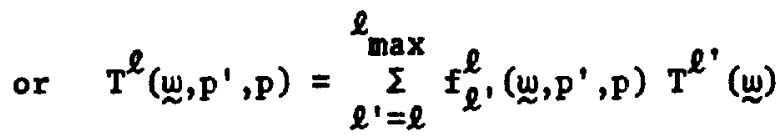


where $f_{\ell^{\prime}}^{\ell}\left(\underset{\sim}{w}, p^{\prime}, p\right) \equiv\left(\frac{2 \ell^{\prime}+1}{2}\right) \int_{-1}^{1} d(\cos \theta) d_{00}^{\ell}(\cos \theta) d_{00}^{\ell^{\prime}}\left(\frac{p^{\prime} p}{p^{2}} \cos \theta\right)$

and $d_{00}^{\ell}(y)$ is given by Eq. A-2 for all vaiues of the variable $y(y=$

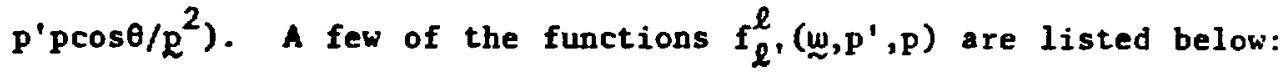

$$
\begin{array}{ll}
f_{0}^{0}=1 & f_{1}^{0}=0 \\
f_{1}^{1}=\left(\frac{P^{\prime} p}{R^{2}}\right) & f_{2}^{1}=0 \\
f_{2}^{2}=\left(\frac{P^{\prime} p}{R^{2}}\right)^{2} &
\end{array}
$$$$
f_{2}^{0}=\frac{5}{2}\left(-1+\left(\frac{p^{\prime} p}{p^{2}}\right)^{2}\right)
$$

In general,

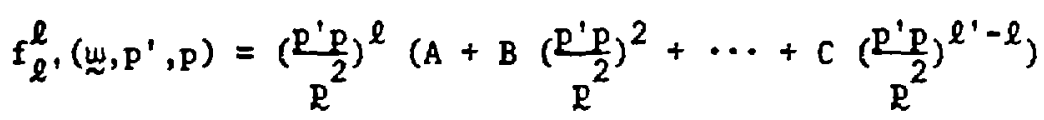

where $A, B, \cdots, C$ are constants,

and $\quad f_{\ell}^{\ell}(\underset{\sim}{w}, \mathfrak{R}, \mathfrak{R})=\delta_{\ell \cdot \ell}$.

This result implies that:

1) At $\mathcal{L} \rightarrow 0$, the on-shiell $T$ function, $T^{\ell '}(\underset{\sim}{)})$, must approach zero at least as fast as $Q^{2 \ell^{\prime}}$ so that the off-energy-shell $T$ function $\mathrm{T}^{l}\left(\underset{\sim}{w}, \mathrm{p}^{\prime}, \mathrm{p}\right)$ will not be divergent in this limit.

2) At $p^{\prime}$ or $p^{\rightarrow} \rightarrow$, the off-energy-shell $T$ function, $T^{l}\left(\underset{\sim}{\sim}, p^{\prime}, p\right)$, is proportional to $\left(p^{\prime} p\right)^{\ell}$. 
3) At $p^{\prime}$ or $p+\infty$, the off-energy-shell $T$ function will be divergent - unless a more general off-shell continuation procedure is adopted.

The first two of these implications are expected and are well known results of nonrelativistic scattering theory. 60 The third implication, however, is an undesireable aspect of the continuation procedure that has been developed here. But, an appealing property of $\mathrm{Eq} .5-74$ is that it implies that the far off-shell behavior of the function $T^{\ell}\left(\underset{\sim}{w}, p^{\prime}, p\right)$ is determined from $T^{\ell^{\prime}}(\underset{\sim}{w})$ with $\ell^{\prime} \geqq \ell$. This is, in fact, reasonable because the off-energy-shell $T$ function should become sensitive to the short-range behavior of the twoparticle force at large momenta. This behavior is embodied in the higher partial waves of the on-shell $\mathrm{T}$ function. (Note: The dependence of the low-order partial-wave amplitudes on the higherorder partial-wave amplitudes is also reguired by the fact that the continuation of the on-shell function must be done before the partial-wave decomposition is performed. That is, an assumption as to the form of the off-energy-shell $T$ function, which is considered as a function of the Lorentz invariants of the system, is made first. Given the off-energy-shell function, one can then consider angular-momentum expansions in terms of the angle $\theta_{p^{\prime}} p$ ) 
To overcome the pathological behavior of $T^{\ell}\left(\underset{\sim}{w}, p^{\prime}, p\right)$ at large $p^{\prime}$ and $p$, there are essentially two methods that can be applied. The first method is to write the off-energy-shell $T$ function as

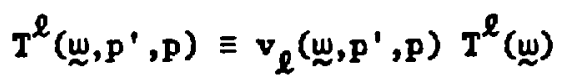

with $v_{\ell}(\underset{w}{w}, \mathfrak{R}, \mathfrak{R})=1$. One can then essume that $v_{\ell}\left(\underset{\sim}{w}, p^{\prime}, p\right) \rightarrow 0$ at $p^{\prime}$ or $p \rightarrow 0\left(e . g,, v_{\ell}\left(w_{w}, p^{\prime}, p\right)=\left(p^{\prime} p / p^{2}\right)^{\ell} \exp \left(-\left(p^{\prime 2}+p^{2}-2 p^{2}\right) / \alpha^{2}\right)-\right.$ see Ref. 55 and references therein). This method, which is extensively used in medium-energy physics, yields an off-energy-shell $T$ function that is convergent in the asymtotic limit. But, this method does not determine the function $v_{\ell}\left(\underset{\sim}{w}, p^{\prime}, p\right)$ in norasymtotic regions of phase space, and it does not explicitly show the dependence of the function $T^{\ell}\left(\underset{w}{w}, p^{\prime}, p\right)$ on higher partial waves. The second method, which will be discussed in $\$ 5.6 .2$, is to develop a dispersion theoretic theory of the off-energy-shell $T$ function. This method can be used to construct the off-energy-shell $T$ function and the "vertex form factor" $v_{\ell}\left(\underset{\sim}{w}, p^{\prime}, p\right)$ from on-shell (i.e., experimental) scattering data. 


\subsubsection{A Dispersion-Theoretic Off-Energy-Shell T Function: Spin O}

In the previous subsection of this chapter, a phenomenological method for continuing an on-shell function into off-energyshell regions of phase space was developed, and it was shown that if this method were applied directly to the on-shell $T$ function then a pathological off-energy-shell $T$ function would result. In this subsection, these methods will again be applied; but, this time they will be applied to the following relation:

$$
T\left(\underset{\sim}{w}, \cos \theta_{p^{\prime} p^{\prime}}\right)-T\left(\underset{\sim}{w}, \cos \theta_{p^{\prime} p^{\prime}}\right)^{\dagger} \equiv-2 \pi i F\left(\underset{\sim}{\omega}, \cos \theta_{p^{\prime} p^{\prime}}\right)
$$

In the fixed $\Delta^{\prime}: \Delta$ model (Eq. 5-68), a continuation of the function $F\left(\underset{\sim}{w}, \cos \theta_{p^{\prime}}\right)$ then implies that

$$
\begin{aligned}
& T\left(\underset{\sim}{w}, p^{\prime}, p, \cos \theta_{p^{\prime} p^{\prime}}\right)-T\left(\underset{\sim}{w}, p^{\prime}, p, \cos \theta_{p^{\prime} p}\right)^{\dagger} \\
& =-2 \pi i F\left(\underset{w}{, P^{\prime} p} \frac{p^{2}}{R^{2}} \cos P^{\prime} P^{\prime}\right)
\end{aligned}
$$

In the total-angular-momentum representation, this equation becomes

$$
\begin{aligned}
& T^{\ell}\left(\underset{\sim}{w}, p^{\prime}, p\right)-T^{\ell}\left(\underset{\sim}{w}, p^{\prime}, p\right)^{\dagger} \\
& =-(2 \pi)^{2} i \int_{-1}^{1} d(\cos \theta) d_{00}^{\ell}(\cos \theta) F\left(\underset{\sim}{w}, \frac{p^{\prime} p}{R^{2}} \cos \theta\right)
\end{aligned}
$$


To proceed further, an assumption as to the $x$ dependence of the function $F(\underset{\sim}{w}, x)$ must be ade. One possibility is to assume that this function is equal to the following "partial-wave" series:

$$
F(\underset{\sim}{w}, x)=\sum_{l=0}^{\infty}\left(\frac{2 \ell+1}{4 \pi}\right) d_{00}^{l}(x) F^{\ell}(\underset{\sim}{)})
$$

With this ansatz Eq. 5-83 becomes

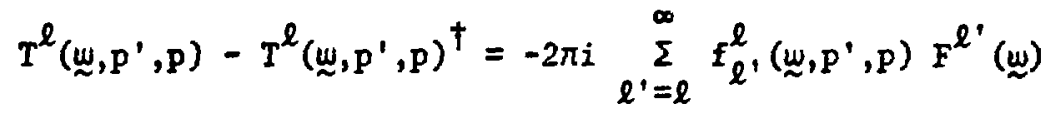

where $F^{l}(\underset{\sim}{w})=\frac{i}{2 \pi}\left\{T^{l}(\underset{\sim}{)})-T^{l}(\underset{\sim}{)})^{\dagger}\right\}$

and the functions $f_{\ell^{\prime}}^{\ell}\left(\underset{w}{w}, p^{\prime}, p\right)$ are defined and discussed in Eqs. 5-75 through 5-78. (Note: This ansatz, which implies that low-order off-shell partial wave amplitudes are dependent on higher-order patial-wave amplitudes, is also suggested by the analysis of Namyslowski. ${ }^{54}$ )

A dispersion relation can now be written for $T^{\ell}\left(\underset{\sim}{W}, P^{\prime}, p\right)$; but, as in Eq. 5-74, the resultant off-energy-shell $T$ function would be divergent at $p^{\prime}$ or $p \rightarrow \infty$. One method of overcoming this problem, which is suggested by the Chew-Low theory of the pion-nucleon interaction, 55 is to modify Eq. 5-81 such that the divergence at large values of $p^{\prime}$ and $p$ is eliminated. That is, one could assume 
that

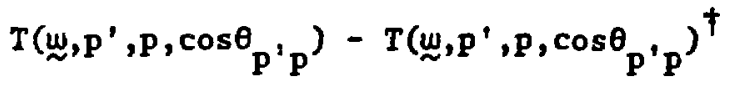

$$
\begin{aligned}
& =-2 \pi i v\left(\underset{\sim}{\omega}, p^{\prime}, p\right) F\left(\underset{\sim}{w}, \frac{p^{\prime} p}{p^{2}} \cos \theta_{p^{\prime} p^{\prime}}\right)
\end{aligned}
$$

with $v\left(\underset{\sim}{w}, p^{\prime}, p\right) \rightarrow 0$ at $p^{\prime}$ or $p \rightarrow \infty$

$$
v\left(\underset{\sim}{w}, p^{\prime}, p\right) \rightarrow \text { constant at } p^{\prime} \text { or } p \rightarrow 0
$$

and $\quad v(\underset{w}{w}, R, R)=1$.

The function $v\left(\underset{\sim}{w}, \mathrm{p}^{\prime}, \mathrm{p}\right)$ will be called a "form factor". Eq. 5-85 is then satisfied by

$$
\begin{aligned}
T^{\ell}\left(\underset{\sim}{w}, p^{\prime}, p\right) & =v^{\ell}\left(\underset{\sim}{w}, p^{\prime}, p\right)+\int_{0}^{\infty} d p^{\prime \prime}\left(\frac{w^{\prime} p^{\prime \prime}}{w_{1}^{\prime} w_{2}^{\prime \prime}}\right)\left(2 w^{\prime}\right) \frac{1}{w^{2}-w^{\prime}{ }^{2}-i \eta} \\
& \times v\left(w^{\prime}, p^{\prime}, p\right) \sum_{\ell^{\prime}=\ell}^{\infty} f_{\ell^{\prime}}^{\ell}\left(w^{\prime \prime}, p^{\prime}, p\right) F^{\ell^{\prime}}\left(w^{\prime \prime}\right)
\end{aligned}
$$

where $v^{\ell}\left(\underset{\sim}{w}, p^{\prime}, p\right)=v^{\ell}\left(\underset{\sim}{w}, p^{\prime}, p\right)^{\dagger}$. (Note: $v^{\ell}\left(\underset{\sim}{w}, p^{\prime}, p\right)$ is included to account for possible pole terms in the off-shell $T$ function - for example, see Ref. 55.) The function $v\left(w^{\prime \prime}, p^{\prime}, p\right)$ can then be determined by requiring a best fit to the on-shell $T$ function. For example, it is found that a reasonable form for this function in the case of pion-nucleon scattering is

$$
\nabla\left(\underset{\sim}{w}, p^{\prime}, p\right)=e^{-\left(p^{\prime 2}+p^{2}-2 p^{2}\right) / \alpha^{2}}
$$


where $\alpha$ is a constant. The value of $\alpha$ is then adjusted to obtain a reasonable fit to experiental scattering data.

Note 1: In Ref. 55, only $\ell=1$ partial waves are considered.

Note 2: According to the analysis that has been presented here, there is no justification for making the form factor $\ell$ dependent. However, if the reaction mechanism is believed to be different in each partial wave (e.g., if one particular partial-wave is dominated by a resonance), then one may assume that these differences are reflected in the form factor. That is, one may replace $v\left(w^{\prime \prime}, p^{\prime}, p\right)$ in Eq. 5-86 with $v_{2}\left(w^{\prime} ', p^{\prime}, p\right)$.

\subsubsection{Phenomenology with Spin}

The methods that were developed in $\$ \$ 5.6 .1$ and 5.6 .2 can also be applied to the two-body problen with spin. In particular, the rotation that is defined in Eq. 5-63 can be applied to the off-energy-shell $M$ function to obtain:

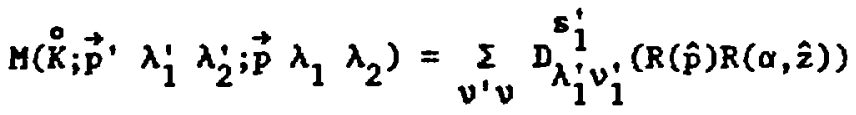

$$
\begin{aligned}
& \times D_{\lambda_{2}^{\prime} v_{2}^{\prime}}^{s_{2}^{\prime}}(R(\hat{p}) R(\alpha, \hat{z})) H\left(\hat{K}_{i r}\left(\theta_{p^{\prime} p}, \hat{y}\right) \vec{p}_{(z)}^{\prime} v_{1}^{\prime} v_{2}^{\prime} ; \vec{p}_{(z)} v_{1} v_{2}\right) \\
& \times D_{v_{1} \lambda_{1}}^{s_{1}}\left((R(\hat{p}) R(\alpha, \hat{z}))^{-1}\right) D_{v_{2} \lambda_{2}}^{s_{2}}\left((R(\hat{p}) R(\alpha, \hat{z}))^{-1}\right)
\end{aligned}
$$


where $M\left(\dot{K}_{;} r\left(\theta_{p^{\prime}} p_{1} \hat{y}\right) \vec{p}_{(z)}^{\prime} v_{1}^{\prime} v_{2}^{\prime} ; \vec{p}_{(z)} v_{1} v_{2}\right)$ is a function of Lorentz invariants only. As in \$5.6.1, an off-energy-shell continuation of the on-shell $M$ function can then be developed by considering this function in atol-angular-momentun representation.

For example, in the helicity-total-angular-monentum representation, the $M$ function is (cf., Eqs. 5-16 and 5-88)

$$
\begin{aligned}
& \mu^{j}\left(\underset{\sim}{w} ; p^{\prime} \lambda_{1}^{\prime} \lambda_{2}^{\prime} ; p \lambda_{1} \lambda_{2}\right)=2 \pi \int_{-1}^{l} d(\cos \theta) d_{\lambda_{1}-\lambda_{2}, \lambda_{1}-\lambda_{2}^{\prime}}^{j}(\theta)
\end{aligned}
$$

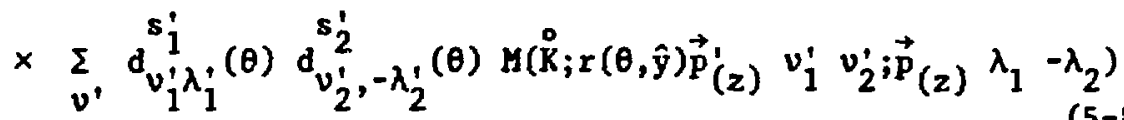

or

$$
\begin{aligned}
& M\left(\dot{K}, r(\theta, \hat{y}) \vec{p}_{(z)}^{\prime} \lambda_{1}^{\prime} \lambda_{2}^{\prime} ; \vec{p}_{(z)} \lambda_{1} \lambda_{2}\right)=\sum_{j v^{\prime}}\left(\frac{2 j+1}{4 \pi}\right) d_{\lambda_{1}+\lambda_{2}, v_{1}^{\prime}+v_{2}^{\prime}}^{j}(\theta)
\end{aligned}
$$

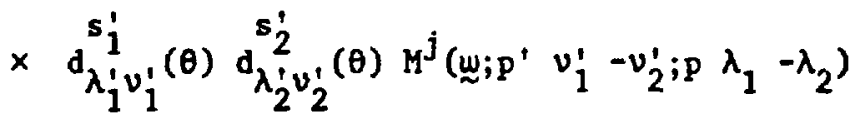

The I function (Eq. 5-29) satisfies equations that are identical to these (i.e., $M$ can be replaced by $T$ in Eqs. 5-89 and 5-90), and

$$
\begin{aligned}
& T\left(\dot{K}, r(\theta, \hat{y}) \vec{p}_{(z)}^{\prime} \lambda_{1}^{\prime} \lambda_{2}^{\prime} ; \vec{p}_{(2)} \lambda_{1} \lambda_{2}\right)=\sum_{v^{\prime} \mu^{\prime}} d_{\lambda_{1}^{\prime} v_{1}^{\prime}}^{s_{1}^{\prime}}(\theta) d_{\lambda_{2}^{\prime} v_{2}^{\prime}}^{s_{2}^{\prime}}(\theta) \\
& \times e^{-\frac{1}{2}\left(v_{1}^{\prime} x_{1}^{\prime}-v_{2}^{\prime} x_{2}^{\prime}-\lambda_{1} x_{1}+\lambda_{2} x_{2}\right)} d_{\mu_{1}^{\prime} v_{1}^{\prime}}^{s}(\theta) d_{\mu_{2}^{\prime} v_{2}^{\prime}}^{s} \\
& H\left(\stackrel{0}{K} ; r(\theta, \hat{y}) \vec{p}_{(z)}^{\prime} \mu_{1}^{\prime} \mu_{2}^{\prime} ; \vec{p}_{(z)} \lambda_{1} \lambda_{2}\right)
\end{aligned}
$$


Now, because the $T$ function is intimately related to the on-shell reaction amplitude (see 55.3 ), it is convenient to use the $T$ function, rather than the $M$ function, in the development of a phenomenology. Indeed, this is possible because (see Eqs. 1-41 and 2-21)

$$
\begin{aligned}
\sum_{v^{\prime}} d_{\lambda^{\prime} v^{\prime}}^{s^{\prime}}(\theta) e^{-\frac{1}{2} v^{\prime} x^{\prime}} d_{\mu^{\prime} v^{\prime}}^{s^{\prime}},(\theta) & =D_{\lambda^{\prime} \mu^{\prime}}^{s^{\prime}}\left(R(\theta, \hat{y}) Z\left(x^{\prime}\right) R(\theta, \hat{y})^{-1}\right) \\
& =D_{\lambda^{\prime} \mu^{\prime}}^{s^{\prime}}\left(I\left(x^{\prime}, \hat{\mathrm{o}}\right)\right)
\end{aligned}
$$

where $\hat{\mathrm{n}}=(\cos \theta, 0, \sin \theta)$

and $\quad L\left(X^{\prime}, \hat{n}\right)=\cosh \frac{x^{\prime}}{2} \sigma_{0}-\hat{n} \cdot \vec{\sigma} \sinh \frac{x^{\prime}}{2}$

This result implies that no square-root branch points are introduced in the conversion from the $M$ function to the $T$ function in Eqs. 5-89 and 5-90; hence, if the $M$ function is an analytic function of the invariants then so is the $T$ function.

Eq. 5-90 (written in terms of the $T$ function) can then be used as in Eq. 5-73 to continue the on-shell $T$ function to offenergy-shell values of the four-momenta; but, the $d$ matrices in Eq. 5-90 imply that the continued function could contain square-root singularities. That is, it appears that the factors $(1 \pm \cos \theta)^{\frac{1}{2}}$, which arise from the half-angle functions in the d atrices (see Eq. A-2), introduce square-root branch points that must be dealt with when $\cos \theta$ is replaced by $p^{\prime} p \cos \theta / \mathbb{R}^{2}$ in the fixed $\Delta^{\prime} \cdot \Delta$ nodel. This 
problem, however, does not exist as can be seen when Eqs. 5-89 and 5-90 are converted to the es-total-angular-momentum representation. In that representation, these equations are (see Eqs. 5-17 and A-7)

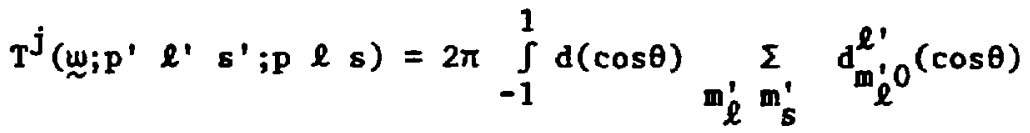

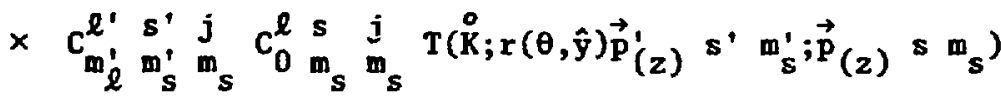

and

$$
\begin{aligned}
& T\left(\stackrel{\circ}{\circ} r(\theta, \hat{y}) \vec{p}_{(z)}^{\prime} s^{\prime} m_{s^{\prime}}^{\prime} \vec{p}_{(z)} s m_{s}\right)=\sum_{\ell^{\prime} m_{\ell}^{\ell}}\left(\frac{2 j+1}{4 \pi}\right) d_{m_{\ell}^{\prime} 0^{\prime}}^{\ell^{\prime}}(\cos \theta)
\end{aligned}
$$

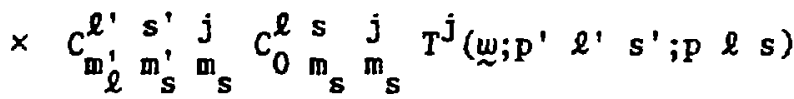

where

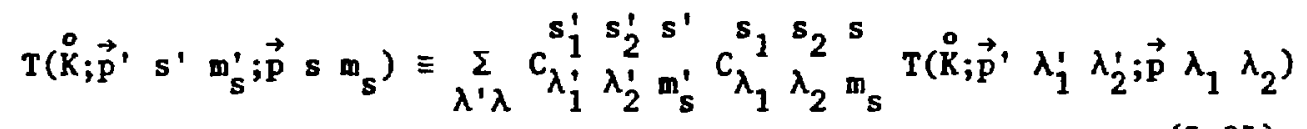

and $\mathrm{d}_{\mathrm{m}_{\ell}^{\prime}{ }^{\prime}}^{\ell^{\prime}}(\cos \theta)$ is a polynomial in the variable $\cos \theta$.

Thus, in the helicity representation there is an illusory problem with square-root singularities that can be remedied by expressing the $T$ function in the $2 s$ representation. In the remainder of this section, only the $2 s$ representation will be used; however, conversion back to the helicity representation is always possible. 
(Note: There is an abundance of literature concerning the location of kinematic singularities in helicity amplitudes? 20,21 The procedure that has been used here most closely resembles that of Ref. 20.)

The methods that were developed in $\$ 5.6 .1$ can now be applied with little modification to the $T$ functions in Egs. 5-93 and 5-94. In particular, the continuation method that was described in Eqs. 5-72 through 5-78 yields the following results:

$$
\begin{aligned}
& T\left(K ; r(\theta, \hat{y}) \vec{p}_{(z)}^{\prime} s^{\prime} m_{s^{\prime}}^{\prime} \vec{p}_{(z)} s m_{s}\right)=\sum_{\substack{j \ell \\
\ell^{\prime} m_{\ell}^{\prime}}}^{\sum}\left(\frac{2 j+1}{4 \pi}\right) d_{m_{\ell}^{\prime} O^{\prime}}^{\ell^{\prime}}\left(\frac{p^{\prime} p}{L^{2}} \cos \theta\right)
\end{aligned}
$$

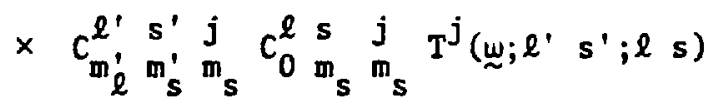

or

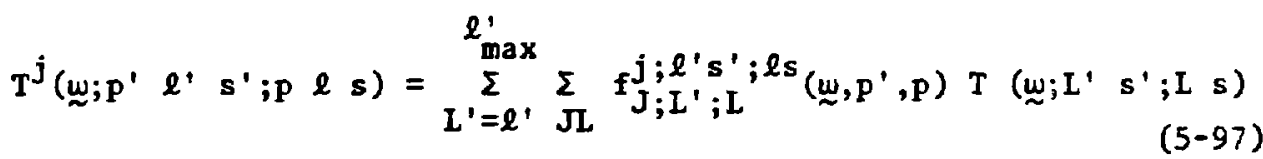

where

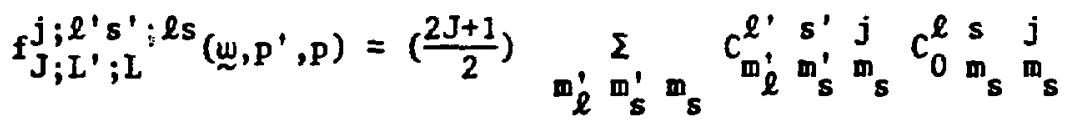

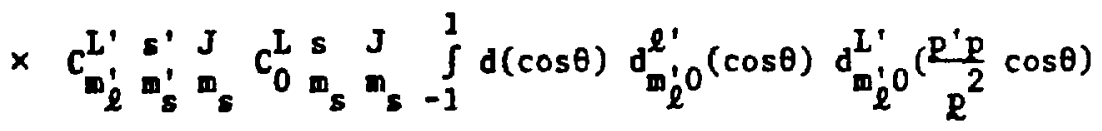

$$
\begin{aligned}
& =\left(\frac{P^{\prime} P}{R^{2}}\right)^{\ell^{\prime}}\left(A+B\left(\frac{P^{\prime} P}{R^{2}}\right)+\cdots+C\left(\frac{P^{\prime} P}{R^{2}}\right)^{L^{\prime}-\ell^{\prime}}\right)
\end{aligned}
$$


where $A, B, \cdots, C$ are constants, and $\mathrm{f}_{J ; L^{\prime} ; L^{j}}^{j ; \ell^{\prime} s^{\prime} ; \ell s}\left(\underset{w, L, P)}{w}=\delta_{j J} \delta_{\ell^{\prime} L}, \delta_{\ell L}\right.$

These equations then imply that:

1) At $Q \rightarrow 0$, the on-shell $T$ function $T^{J}$ (్ㅣ; $\left.L^{\prime} s^{\prime} ; L s\right)$ must approach zero at least as fast as $\mathrm{Q}^{2 \mathrm{~L}}$ 'so that the off-energy-shell T function is not divergent in this limit.

2) At $p^{\prime}$ or $p \rightarrow 0$, the off-energy-shell $T$ function

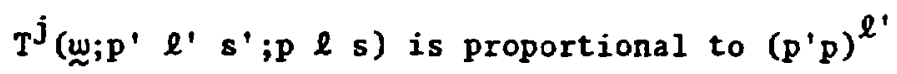

3) At $p^{\prime}$ or $p \rightarrow \infty$, the off-energy-shell $T$ function will be divergent - unless a more general off-shell continuation procedure is adopted.

Similarly, the results of $\$ 5.6 .2$ can be applied to overcome the divergence problem, and the dispersion relation for the $T$ function in the $\ell s$-total-angular-momentum representation can be obtained by inspection of Eq. 5-86. The result is

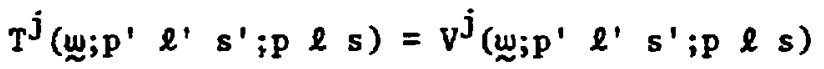

$$
\begin{aligned}
& +\int_{0}^{\infty} \mathrm{dp} p^{\prime \prime}\left(\frac{w^{\prime \prime} p^{\prime \prime}}{w_{1}^{\prime} w_{2}^{\prime \prime}}\right)\left(2 w^{\prime \prime}\right) \frac{1}{w^{2}-w^{\prime \prime 2}+i \eta} \\
& \times v\left(w^{\prime \prime}, p^{p}, p\right) \sum_{L^{t}=\ell^{\prime}}^{\infty} \sum_{J} f_{J^{\prime} ; L^{\prime} ; L^{\prime}}^{j ; \ell^{\prime} s^{\prime} ; \ell s}\left(w^{\prime}, P^{\prime}, p\right) F^{J}\left(w^{\prime}, L^{\prime} s^{\prime}, L s\right)
\end{aligned}
$$




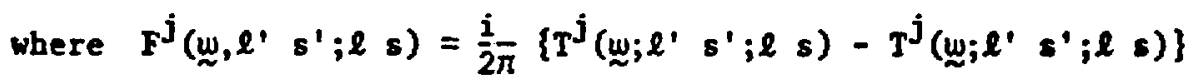

and $v\left(\underset{w}{w}, p^{\prime}, p\right)$ is a form factor.

There is, however, one undesireable aspect of the phenomenological method that has been presented thus far. This is the esymmetry between the initial and final states, which is caused by the particular choice of the on-shell function that is being continued off-shell. In Eq. 5-88, this function was defined such that the three-vector $\vec{p}$ was parallel to the $\hat{z}$ axis; whereas, $\vec{p}$ ' was at an angle $\theta_{p^{\prime} p}$ from the $\hat{z}$ axis in the $\hat{x}-\hat{z}$ plane. Another function could bave been defined that would have $\vec{p}$ ' parallel to the $\hat{z}$ axis and $\vec{p}$ in the $\hat{x}-\hat{z}$ plane. That is, one could let

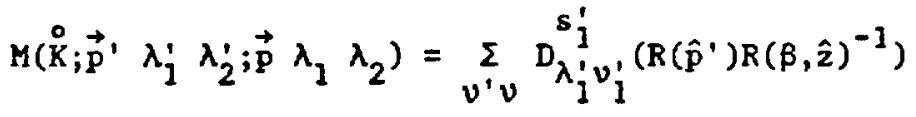

$$
\begin{aligned}
& \times D_{\lambda_{2}^{\prime} v_{2}^{\prime}}^{s_{2}^{\prime}}\left(R\left(\hat{p}^{\prime}\right) R(\beta, \hat{z})^{-1}\right) M\left({\stackrel{K}{;} ; \vec{p}_{(z)}^{\prime}}^{\prime} v_{1}^{\prime} v_{2}^{\prime} ; r\left(\theta_{p^{\prime} p^{,}}, \hat{y}\right)^{-1} \vec{p}_{(z)} v_{1} v_{2}\right) \\
& \times D_{v_{1} \lambda_{1}}^{s_{1}}\left(\left(R\left(\hat{p}^{\prime}\right) R(\beta, \hat{z})^{-1}\right)^{-1}\right) D_{v_{2} \lambda_{2}}^{s_{2}}\left(\left(R\left(\hat{p}^{\prime}\right) R(\beta, \hat{z})^{-1}\right)^{-1}\right)
\end{aligned}
$$

This definition results in the roles of the initial and final states being interchanged throughout the analysis of this section. Thus, one way to remedy the asymetry problem is to simply add the equations that result from these two aethods and divide by two. The net result of this manipulation is to replace the function 
$f_{J ; L^{\prime} ; L}^{j ; \ell^{\prime} s^{\prime} ; \ell s}\left(\underset{\sim}{w}, p^{\prime}, p\right)$ in Eqs. 5-97 and 5-100 with the following function:

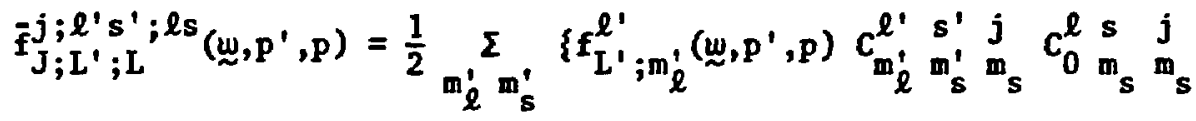

$$
\begin{aligned}
& m_{\ell} m_{s}
\end{aligned}
$$

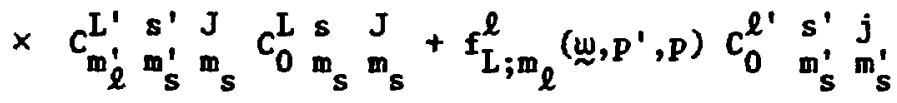

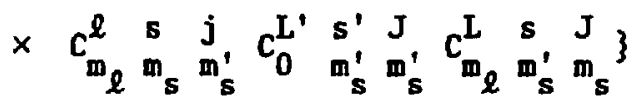

where

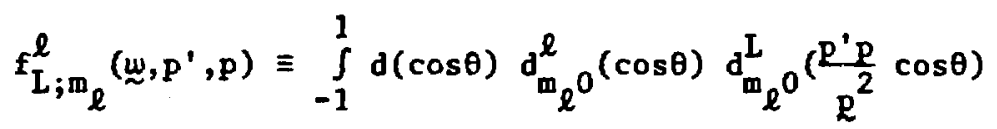

This off-shell continuation method implies that:

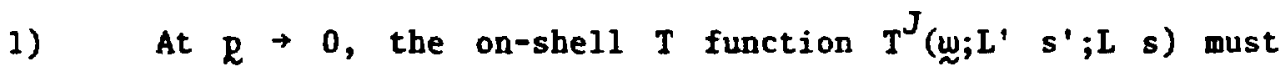
approach zero at least as fast as $\mathrm{p}^{\max \left(2 L^{\prime}, 2 L\right)}$ where $\max \left(2 L^{\prime}, 2 L\right)$ is the larger of $2 L^{\prime}$ and $2 L$.

2) At $p^{\prime}$ or $p \rightarrow 0$, the off-energy-shell $T$ function

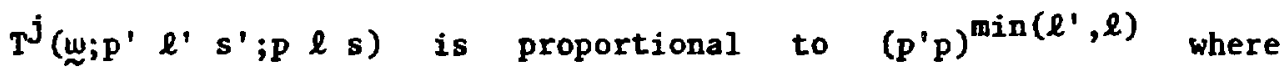
min $\left(\ell^{\prime}, l\right)$ is the smaller of $\ell^{\prime}$ and $l$. 
These results also imply that the off-energy-shell $T$ function can be written as

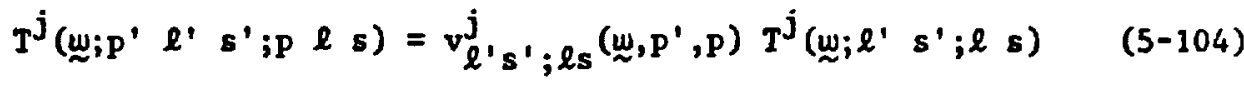

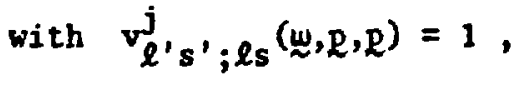

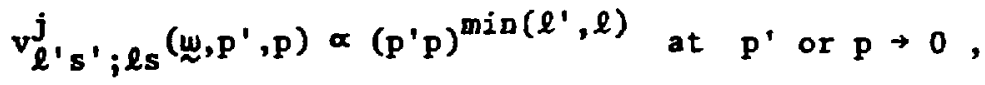

and $\left.\quad v_{\ell^{\prime} s^{\prime} ; \ell s}^{j} \underset{\sim}{w}, p^{\prime}, p\right) \rightarrow 0$ at $p^{\prime}$ or $p \rightarrow \infty$. 
Chapter 6

INTRODUCTION TO THE RELATIVISTIC THREE-BODY PROBLEM

A system of three interacting particles is the simplest system in which the off-shell theory of Chapters 4 and 5 can be applied. A complete discussion of the equations for a relativistic description of a three-body system will not be attempted here; however, some of the basic ingredients for those equations will be considered.

One of the most awkward things about the three-body problem is establishing a reasonable notation. This notation and the kinematics of the three-particle system are discussed in $\$ 6.1$. In $\$ 6.2$, the concepts that were developed during the study of the state vectors for one- and two-particle systems are extended to the threeparticle system, and the three-body recoupling coefficient, which was first considered by Wick, 48 is defined. This method of defining the recoupling coefficient is, however, only valid for on-shell 
values of the four-momenta. A different method of defining it must be considered when it is to be used in an off-shell environment.

The simplest way to construct the off-mass-shell recoupling coefficient is to consider an example. In $\$ 6.3$, a one-particle exchange diagram is considered and the recoupling coefficient is derived in the $M$ function formalism. This diagram is used because it involves the off-mass-shell recoupling coefficient in regions of the two-body subenergy variables where real Lorentz transformations to the center-of-mass of the two-body subsystems exist. This advantage is not realized in the complete three-body problem (i.e., under an iteration of the one-parti(:le exchange diagram), but it is expected that methods can be developed that will circumvent this problem. This aspect of the relativistic three-body problem is currently under study and will not be discussed further here.

The off-mass-shell $T$ function recoupling coefficient is defined in $\$ 6.4$, and it is shown that this coefficient can be concidered as an off-mass-shell extension of the on-shell recoupling coefficient that was defined in $\$ 6.2$. Finally, isospin and the three-body isospin recoupling coefficient are briefly discussed in 56.5. It is shown that the isospin aspects of the three-body problem are easily incorporated into the formalism that is presented here. 


\subsection{The Kinematics of Three-Particle States}

Consider three particles with momenta $\overrightarrow{\mathrm{P}}_{1}, \overrightarrow{\mathrm{P}}_{2}$, and $\overrightarrow{\mathrm{P}}_{3}$ and energies $E_{1}, E_{2}$, and $E_{3}\left(E_{i} \equiv\left(\vec{P}_{i}{ }^{2}+m_{i}{ }^{2}\right)^{\frac{1}{2}}\right)$. Eq. 1-31 implies that the four-momentum of each particle $\left(P_{i} \equiv\left(E_{i}, \vec{P}_{i}\right)\right)$ in the reference frame where $\overrightarrow{\mathrm{P}}_{1}+\overrightarrow{\mathrm{P}}_{2}+\overrightarrow{\mathrm{P}}_{3}=\overrightarrow{\mathrm{P}}$ is related to the four-momentum in the over-all-center-of-mass (OACM) reference frame (i.e., that frame where $\overrightarrow{\mathrm{P}}_{1}+\overrightarrow{\mathrm{P}}_{2}+\overrightarrow{\mathrm{P}}_{3}=0$ ) by

$$
\begin{aligned}
& \vec{P}_{i}=\vec{P}_{i}^{\prime}+\gamma \vec{\beta}\left(\frac{\gamma}{\gamma+1} \vec{\beta} \cdot \vec{P}_{i}+E_{i}^{\prime}\right) \\
& E_{i}=\gamma\left(E_{i}^{\prime}+\vec{\beta} \cdot \vec{P}_{i}^{\prime}\right) \quad: \gamma=\left(1-\vec{\beta}^{2}\right)^{-\frac{1}{2}}
\end{aligned}
$$

where the prime denotes quantities in the $\mathrm{OACM}$ reference frame. The velocity of the Lorentz transformation is

$$
\vec{\beta}=\frac{\vec{P}}{E} \quad \text { and } \quad \gamma=\frac{E}{E^{\prime}}
$$

where $\overrightarrow{\mathrm{P}} \equiv \overrightarrow{\mathrm{P}}_{1}+\overrightarrow{\mathrm{P}}_{2}+\overrightarrow{\mathrm{P}}_{3}$ and $E \equiv E_{1}+E_{2}+E_{3}$. The relative threemomentum between the $(i, j)$ pair of particles and $k$ th particle, $\vec{q}_{k}$, is defined to be the total momentum of the $(i, j)$ pair in the $O A C M$ reference frame $\left(\vec{q}_{k}=\overrightarrow{\mathbf{P}}_{i}+\overrightarrow{\mathbf{P}}_{j}=-\overrightarrow{\mathbf{P}}_{k}\right)$.

Now consider the. $(i, j)$ pair of particles. Equation 1-31 implies that the four-momentum of each of these particles in the OACM reference frame is related to the four-momentum in their 
center-of-mass reference frame $\left(\mathrm{CM}_{k}\right)$ by

$$
\begin{aligned}
& \vec{p}_{i}^{\prime}=\vec{p}_{i}^{\prime}+\gamma_{k} \vec{\beta}_{k}\left(\frac{\gamma_{k}}{\gamma_{k}+1} \vec{\beta}_{k} \cdot \vec{\beta}_{i}^{\prime}+E_{i}^{\prime}\right) \\
& E_{i}^{\prime}=\gamma_{k}\left(E_{i}^{\prime \prime}+\vec{\beta}_{k} \cdot \vec{P}_{i}^{\prime}\right)
\end{aligned}
$$

where the double-prime denotes quantities in the $\mathrm{CM}_{k}$ reference frame. The velocity of the Lorentz transformation is

$$
\vec{\beta}_{k}=\frac{\vec{P}_{(k)}^{\prime}}{E_{(k)}^{\prime}}=\frac{\vec{q}_{k}}{E_{(k)}^{\prime}} \text { and } \quad \gamma_{k}=\frac{E_{(k)}^{\prime}}{E_{(k)}^{\prime}}
$$

where $\vec{P}_{(k)} \equiv \vec{P}_{i}+\vec{P}_{j}$ and $E_{(k)} \equiv E_{i}+E_{j}$. The relative threemomentum $\overrightarrow{\mathrm{p}}_{k}$ is defined to be the momentum of particle $i$ in the $\mathrm{CM}_{k}$ reference frame $\left(\vec{p}_{k}=\vec{p}_{i}^{\prime}=-\vec{p}_{j}^{\prime}\right)$.

The following notation will also be used:

$$
\begin{aligned}
& \vec{k}_{i(k)} \equiv \vec{P}_{i}^{\prime}=\vec{p}_{k} \\
& \overrightarrow{\mathbf{k}}_{j(k)} \equiv \overrightarrow{\mathbf{P}}_{j}^{\prime \prime}=-\overrightarrow{\mathbf{p}}_{k} \\
& w_{i(k)} \equiv E_{i}^{\prime \prime}=\left(\vec{p}_{k}^{2}+m_{i}^{2}\right)^{\frac{1}{2}} \quad w_{j(k)} \equiv E_{j}^{\prime}=\left(\vec{p}_{k}^{2}+m_{j}^{2}\right)^{\frac{3}{2}} \\
& w_{(k)} \equiv w_{i(k)}+w_{j(k)} \\
& \overrightarrow{\mathbf{k}}_{(k)} \equiv \overrightarrow{\mathbf{P}}_{i}+\overrightarrow{\mathbf{F}}_{j}^{t}=\vec{q}_{k} \quad \overrightarrow{\mathbf{K}}_{k} \equiv \overrightarrow{\mathbf{P}}_{k}=-\vec{q}_{k} \\
& W_{(k)} \equiv E_{i}^{\prime}+E_{j}=\left(\vec{q}_{k}^{2}+w_{(k)}\right)^{2} \frac{1}{2} \quad W_{k} \equiv E_{k}^{\prime}=\left(\vec{q}_{k}^{2}+m_{k}^{2}\right)^{\frac{1}{2}} \\
& W \equiv W_{(k)}+w_{k}=\left(E^{2}-\vec{p}^{2}\right)^{\frac{1}{k}}
\end{aligned}
$$


Equations that relate these quantities are easily determined by inspection of Egs. 3-3 through 3-7.

The above results imply that any function of the threemomenta $\vec{P}_{1}, \vec{P}_{2}$, and $\vec{P}_{3}$ can be written as a function of $\vec{P}, \vec{q}_{k}$, and $\vec{p}_{k}$. The Jacobian of the variable change $\left(\vec{P}_{1}, \vec{P}_{2}, \vec{P}_{3}\right)$ to $\left(\vec{P}, \vec{q}_{k}, \vec{p}_{k}\right)$ is most easily determined by defining two $W-G$ relative four-momenta, $\Delta_{k}$ and $\Delta_{(k)}$, as (cf., Eq. 3-8)

$$
\begin{aligned}
& \left(\Delta_{k}\right)_{0} \equiv \frac{1}{W}\left(W_{k} E_{(k)}-W_{(k)} E_{k}\right) \\
& \vec{\Delta}_{k} \equiv \frac{1}{W}\left(W_{k} \vec{P}_{(k)}-W_{(k)} \vec{P}_{k}\right)
\end{aligned}
$$

and

$$
\begin{aligned}
& \left(\Delta_{(k)}\right)_{0} \equiv \frac{1}{w_{(k)}}\left(w_{j(k)} E_{i}-w_{i(k)} E_{j}\right) \\
& \overleftarrow{\Delta}_{(k)} \equiv \frac{1}{w_{(k)}}\left(w_{j(k)} \vec{P}_{i}-w_{i(k)} \vec{p}_{j}\right)
\end{aligned}
$$

where $w, w_{(k)}, w_{k}, w_{(k)}, w_{i(k)}$, and $w_{j(k)}$ are Lorentz invariants; so that, $\Delta_{k}$ and $\Delta_{(k)}$ are indeed four-vectors. These $H-G$ relative four-momenta can also be written as

$$
\Delta_{k}=\frac{1}{2}\left(P_{(k)}-P_{k}\right)-\frac{1}{2 s}\left(m_{(k)}{ }^{2}-m_{k}^{2}\right) P
$$

and

$$
\Delta_{(k)}=\frac{1}{2}\left(P_{i}-P_{j}\right)-\frac{1}{2 \varepsilon_{k}}\left(m_{i}^{2}-m_{j}^{2}\right) P_{(k)}
$$


where $s \equiv P^{2}=w^{2}, s_{k}=P_{(k)}{ }^{2}=w_{(k)}{ }^{2}, m_{(k)}=w_{(k)}$, and $P_{i}, P_{j}, P_{k}, P_{(k)}$ and $P$ are the on-shell four-momenta (i.e., $P_{i}=$ $\left(E_{i}, \vec{P}_{i}\right), P_{(k)}=\left(E_{(k)}, \vec{P}_{(k)}\right)$, etc. $)$

Equations 6-8 and 6-9 can then be used to define the W-G relative four-momenta for arbitrary values of $\left(\mathrm{P}_{1}\right)_{0},\left(\mathrm{P}_{2}\right)_{0}$, and $\left(P_{3}\right)_{0}$. Permitting these additional three degrees of freedom allow one to consider the variable change $\left(P_{1}, P_{2}, P_{3}\right)$ to $\left(P, \Delta_{k}, \Delta_{(k)}\right)$. The Jacobian for this 12-dimensional phase-space change is one,

i.e., $\quad d^{4} P_{1} d^{4} P_{2} d^{4} P_{3}=d^{4} P d^{4} \Delta_{k} d^{4} \Delta_{(k)}$

provided the effective mass of the $(i, j)$ pair (i.e., $\left.m_{(k)}\right)$ is a function of $\Delta_{(k)}$ only. For on-shell values of the four-momenta (see Eqs. 6-13 through 6-15 and note that $\left(p_{k}\right)_{0}=0$ when the particle four-momenta are on-shell),

$$
m_{(k)}=w_{(k)}=\left(\vec{p}_{k}^{2}+m_{i}\right)^{\frac{1}{2}}+\left(\vec{p}_{k}^{2}+m_{j}^{2}\right)^{\frac{1}{2}}
$$

and $\quad \Delta_{(k)}{ }^{2}=-\vec{p}_{k}^{2}$.

Hence, a suitable definition of $m_{(k)}$ for off-mass-shell values of the four-momenta is

$$
m_{(k)} \equiv\left(\left|\Delta(k){ }^{2}\right|+m_{i}{ }^{2}\right)^{\frac{1}{2}}+\left(\left|\Delta(k){ }^{2}\right|+m_{j}{ }^{2}\right)^{\frac{2}{2}}
$$


(Note: The definition of the W-G relative four-monentun that is given in Eq. 6-8 with ${ }^{(k)}$ given by Eq. 6-12 was necessary to obtain a unit Jacobian in Eq. 6-10. A by-product of this definition is that the effective mass of the two-body system, ${ }^{n}(k)$, is independent of the total four-momentum. I therefore expect that if the the two-body interactions are expressed in terms of $\Delta_{k}$ then the resultant three-body theory will be seperable. 61 This question is currently under investigation.)

Finally, the Jacobian for the 9-dimensional phase-space change, $\left(\vec{p}_{1}, \overrightarrow{\mathrm{P}}_{2}, \overrightarrow{\mathrm{P}}_{3}\right)$ to $\left(\overrightarrow{\mathrm{P}}, \vec{q}_{k}, \overrightarrow{\mathrm{p}}_{k}\right)$, is determined by restricting the values of $\left(\mathrm{P}_{1}\right)_{0},\left(\mathrm{P}_{2}\right)_{0}$, and $\left(\mathrm{P}_{3}\right)_{0}$ to their mass-shell values. (see Eq. 3-11)

$$
\begin{aligned}
& d^{3} P_{1} d^{3} P_{2} d^{3} P_{3}=d^{4} P_{1} d^{4} P_{2} d^{4} P_{3} \delta\left(\left(P_{1}\right)_{0}-E_{1}\right) \delta\left(\left(P_{2}\right)_{0}-E_{2}\right) \delta\left(\left(P_{3}\right)_{0}-E_{3}\right) \\
& =8 E_{1} E_{2} E_{3} d^{4} P_{1} d^{4} P_{2} d^{4} P_{3} \delta^{+}\left(P_{1}{ }^{2}-m_{1}{ }^{2}\right) \delta^{+}\left(P_{2}{ }^{2}-m_{2}{ }^{2}\right) \delta^{+}\left(P_{3}{ }^{2}-m_{3}{ }^{2}\right) \\
& =\frac{2 E_{1} E_{2} E_{3}{ }^{W}(k)^{W}}{w_{i}(k)^{w_{j}}(k)^{W}(k)^{W}} d^{4} P^{4} q_{k} d^{4} p_{k} \delta^{+}\left(P^{2}-W^{2}\right) \delta\left(\left(q_{k}\right)_{0}\right) \delta\left(\left(p_{k}\right)_{0}\right) \\
& =\frac{E_{1} E_{2} E_{3}}{E} \frac{{ }^{w}(k)^{W}}{w_{i}(k)^{w}(k)^{W}(k)^{W}} d^{3} P d^{3} q_{k} d^{3} p_{k}
\end{aligned}
$$

where the relative four-momenta, $q_{k}$ and $p_{k}$, have been defined as

$$
q_{k}=\ell\left(x_{p}, \hat{P}\right)^{-1} \Delta_{k}
$$


and $\quad p_{k}=\ell\left(x_{P_{(k)}}, \vec{p}_{(k)}\right)^{-1} \Delta_{(k)}$

$$
=\ell\left(x_{K_{(k)}}, \hat{K}_{(k)}\right)^{-1} \ell\left(x_{P}, \hat{P}\right)^{-2} \Delta_{(k)}
$$

These are real Lorentz transformations provided $P_{0}>|\vec{P}|$ and $\left(P_{(k)}\right)_{0}$ $>\left|\vec{P}_{(k)}\right|$ (or $\left.\left(K_{(k)}\right)_{0}>\left|\vec{K}_{(k)}\right|\right)(c f .$, Eq. 3-12). These requirements are satisfied in Eq. 6-13.

\subsection{The Definition of Three-Particle State Vectors}

The concepts that evolved during the analysis of twoparticle states ( $\$ \$ 3.2$ through 3.4 ) can be applied without modification to an analysis of three-particle states. For example, a three-particle fundamental state vector can be defined as

$$
\begin{aligned}
& \mid \stackrel{\circ}{P}\left[\vec{q}_{k}\left(\vec{p}_{k} \lambda_{i} \lambda_{j}\right)_{z} \lambda_{k}\right]_{z}> \\
& \quad \equiv U\left(L\left(x_{K}, \hat{q}_{k}\right)\right)\left|\stackrel{\circ}{K}_{(k)}\left(\vec{p}_{k} \lambda_{i} \lambda_{j}\right)>\otimes U\left(L\left(x_{K_{k}},-\hat{q}_{k}\right)\right)\right| \dot{k}_{k} \lambda_{k}>
\end{aligned}
$$

Clearly, there are many more ways of defining the three-particle fundamental state vector. A few of these will be discussed later in this section.

A basis of $M^{3}\left(2 x^{3}=X^{1} \otimes N^{1} \otimes N^{1}\right)$ is also constructed in complete analogy with the construction of the $y^{1}$ and $X^{2}$ bases 


$$
\text { i.e., } \quad\left|P\left[\vec{q}_{k}\left(\vec{p}_{k} \lambda_{i} \lambda_{j}\right) \lambda_{k}\right]\right\rangle \equiv U(C(P, \stackrel{\circ}{P}))\left|\stackrel{\circ}{P}\left[\vec{q}_{k}\left(\vec{p}_{k} \lambda_{i} \lambda_{j}\right) \lambda_{k}\right]\right\rangle
$$

and the completeness relation can be determined from Eqs. 2-8 and 6-13.

$$
\begin{aligned}
& 1=\sum_{\lambda} \int \frac{d^{3} p}{2 E} d^{3} q_{k} d^{3} p_{k} \frac{{ }^{w}(k)^{W}}{4 w_{i}(k)^{w} j(k)^{W}(k)^{W}} \\
& \times\left|P\left[\vec{q}_{k}\left(\vec{p}_{k} \lambda_{i} \lambda_{j}\right) \lambda_{k}\right]><P\left[\vec{q}_{k}\left(\vec{p}_{k} \lambda_{i} \lambda_{j}\right) \lambda_{k}\right]\right|
\end{aligned}
$$

The normalization is

$$
\begin{aligned}
& \left\langle P^{\prime}\left[\vec{q}_{k}^{\prime}\left(\vec{p}_{k}^{\prime} \lambda_{i}^{\prime} \lambda_{j}^{\prime}\right) \lambda_{k}^{\prime}\right] \mid P\left[\vec{q}_{k}\left(\vec{p}_{k} \lambda_{i} \lambda_{j}\right) \lambda_{k}\right]\right\rangle=2 E \delta^{3}\left(\vec{P}^{\prime}-\vec{P}\right) \\
& \times\left(\frac{{ }^{4} w_{i(k)^{W}}{ }^{W}(k)^{W}(k)^{W}}{W_{(k)}^{W}}\right) \delta^{3}\left(\vec{q}_{k}^{\prime}-\vec{q}_{k}\right) \delta^{3}\left(\vec{p}_{k}^{\prime}-\vec{p}_{k}\right) \delta_{\lambda_{1} \lambda_{1}} \delta_{\lambda_{2} \lambda_{2}} \delta_{\lambda_{3} \lambda_{3}}
\end{aligned}
$$

Angular momentum bases can then be constructed following the procedures that were developed in \$3.3. For example, a mixed Z/helicity-total-angular-momentum basis state vector is 


$$
\begin{aligned}
& \mid P\left[q_{k} J M\left(p_{k} j_{k} m_{k} \ell_{k} s_{k}\right)_{i j ; 2} \lambda_{k}\right]_{h}>
\end{aligned}
$$

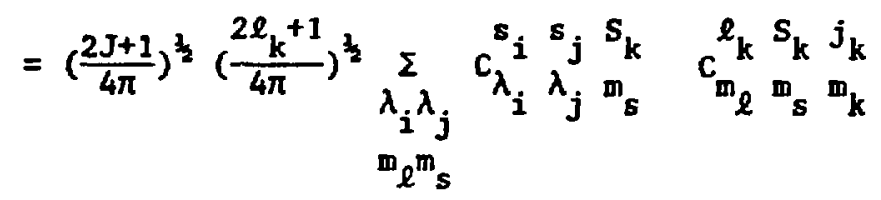

$$
\begin{aligned}
& x \int d^{2} \hat{q}_{k} d^{2} \hat{p}_{k} D_{M, m_{k}-\lambda_{k}}^{J}\left(R\left(\hat{q}_{k}\right)\right)^{*} D_{m_{\ell}{ }^{0}}^{l_{k}}\left(R\left(\hat{p}_{k}\right)\right)^{*}\left|P\left[\vec{q}_{k}\left(\vec{p}_{k} \lambda_{i} \lambda_{j}\right)_{z} \lambda_{k}\right]_{h}\right\rangle
\end{aligned}
$$

where

$$
\begin{aligned}
& \left|P\left[\vec{q}_{k}\left(\vec{p}_{k} \lambda_{i} \lambda_{j}\right)_{z} \lambda_{k}\right]_{b}\right\rangle=U(C(P, \stackrel{P}{P}))\left\{U\left(R\left(\hat{q}_{k}\right) Z\left(x_{\left.y_{(k}\right)}\right)\right)\right. \\
& \times\left[U\left(L\left(x_{k_{i}(k)}, \hat{p}_{k}\right)\right)\left|\hat{k}_{i} \lambda_{i}>\otimes U\left(L\left(x_{k_{j}(k)},-\hat{p}_{k}\right)\right)\right| \hat{k}_{j} \lambda_{j}>\right] \\
& \left.\otimes e^{-i \pi s_{k}} U\left(R\left(\hat{q}_{k}\right) R(\pi, \pi) Z\left(x_{K_{k}}\right)\right) \mid \hat{k}_{k} \lambda_{k}>\right\}
\end{aligned}
$$

The notation is explained in $\$ 3.2$ and the momenta are defined in Eq. 6-5.

Aside from the multiplicity of bases arising from different choices of the complementary set of Lorentz transformations, the particles in the fundamental state vector can be arranged in different ways. For example, particles 1 and 2 could be coupled first and particle 3 coupled to that pair, or particles 2 and 3 could be coupled first and particle 1 coupled to that pair, etc. Bases that are constructed by different orderings of the particles are related 
by "recoupling coefficients". In a helicity-total-angular-momentum basis, the recoupling coefficient will be defined by

$$
\begin{aligned}
& \left\langle P^{\prime}\left[q_{k} J^{\prime} M^{\prime}\left(p_{k} j_{k} m_{k} \lambda_{i}^{\prime} \lambda_{j}^{\prime}\right)_{h} \lambda_{k}^{\prime}\right]_{h}\right| P\left[q_{i} J M\left(p_{i} j_{i} m_{i} \lambda_{j} \lambda_{k}\right)_{h} \lambda_{i}\right]_{h^{\prime}} \\
& \equiv 8 \frac{{ }^{\omega}(k){ }^{w}(j)}{q_{k} p_{k} q_{i} p_{i}} \delta^{4}\left(P^{\prime}-P\right) \delta_{J} J^{\prime} \delta_{M^{\prime} M} \\
& \quad \times c_{J}\left\{W ; q_{k}\left(p_{k} j_{k} m_{k} \lambda_{i}^{\prime} \lambda_{j}^{\prime}\right) \lambda_{k}^{\prime} ; q_{i}\left(p_{i} j_{i} m_{i} \lambda_{j} \lambda_{k}\right) \lambda_{i}\right\} \quad(6-22)
\end{aligned}
$$

A detailed derivation of this recoupling coefficient will be given in $\hat{\S} \hat{s} 6.3,6.4$, and $\bar{E}-\hat{2}$. It wili be showil that $c_{J}$ in at pure tedicily representation is equal to a product of six rotation matrices, whose arguments are functions of the energies and momenta. In a mixed $Z$ /helicity or a pure $Z$ representation, the recoupling coefficient is complicated by the appearance of Clebsch-Gordon coefficients.

Note 1: In all of these bases and in subsequent sections on the $M$ function, the order in which the first two particles are coupled is relevant. The relation between state vectors that use different orderings for these two particles can be determined from the results of \$3.3.3.

Note 2: It will be shown in the next few sections of this chapter that the recoupling coefficient is real. This implies that the coefficient $\langle(i j) k \mid(j k) i\rangle$ is equal to the coefficient $\langle(j k) i \mid(i j) k\rangle$. 


\subsection{The M Function Recoupling Coefficient}

Consider the sequence of interactions that is shown in Fig. 6.1.

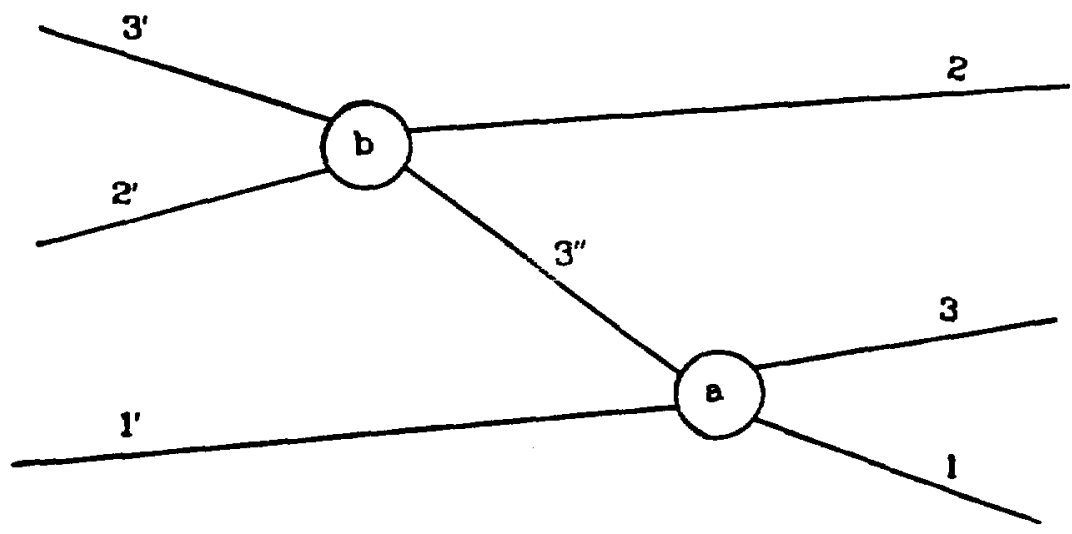

final - initial

Fig. 6.1 A sequence of two interactions in the three-body problem.

This is not a time-ordered sequence and has been drawn with the final state on the left and the initial state on the right to emphasize this fact.

The function that represents this scattering event is determined from the Feyman rules that were presented in \$4.3. 


$$
\begin{aligned}
M\left(P_{1}^{\prime}\right. & \left.\lambda_{1}^{\prime}, P_{2}^{\prime} \lambda_{2}^{\prime}, P_{3}^{\prime} \lambda_{3}^{\prime} ; P_{1} \lambda_{1}, P_{2} \lambda_{2}, P_{3} \lambda_{3}\right) \\
= & \sum_{\lambda_{3}^{\prime}} \int d^{4} P_{3}^{\prime \prime} \frac{1}{P_{3}^{\prime \prime 2}-m_{3}^{2}+i \eta} \\
& \times M_{b}\left(P_{2}^{\prime} \lambda_{2}^{\prime}, P_{3}^{\prime} \lambda_{3}^{\prime} ; P_{2} \lambda_{2}, P_{3}^{\prime \prime} \lambda_{3}^{\prime \prime}\right) M_{a}\left(P_{3}^{\prime \prime} \lambda_{3}^{\prime \prime}, P_{1}^{\prime} \lambda_{1}^{\prime} ; P_{3} \lambda_{3}, P_{1} \lambda_{1}\right) \\
& \times \delta^{4}\left(P_{3}^{\prime}+P_{2}^{\prime}-P_{2}-P_{3}^{\prime \prime}\right) \delta^{4}\left(P_{3}^{\prime \prime}+P_{1}^{\prime}-P_{1}-P_{3}\right)
\end{aligned}
$$

This equation can also be written in terms of the total monentum and the appropriate $W-G$ relative momenta by relabeling the $M$ functions and by changing the integration variables as follows:

$$
\begin{gathered}
\int d^{4} P_{3}^{\prime \prime} \delta^{4}\left(P_{3}^{\prime}+P_{2}^{\prime}-P_{2}-P_{3}^{\prime \prime}\right) \delta^{4}\left(P_{3}^{\prime \prime}+P_{1}^{\prime}-P_{1}-P_{3}\right) \\
=\int d^{4} P^{\prime \prime} d^{4} \Delta_{1}^{\prime \prime} d^{4} \Delta_{(1)}^{\prime \prime} \delta^{4}\left(P_{(1)}^{\prime}-P_{(1)}^{\prime \prime}\right) \delta^{4}\left(P_{1}^{\prime}-P_{1}^{\prime}\right) \\
\left.\times \delta^{4}\left(P_{(2)}^{\prime}\right)-P_{(2)}\right) \delta^{4}\left(P_{2}^{\prime \prime}-P_{2}\right) \\
=\delta^{4}\left(P^{\prime}-P\right) \int d^{4} \Delta_{1}^{\prime} d^{4} \Delta_{(1)}^{\prime} \delta^{4}\left(P_{1}^{\prime}-P_{1}^{\prime \prime}\right) \delta^{4}\left(P_{2}^{\prime \prime}-P_{2}\right)
\end{gathered}
$$


The equation that results from this manipulation is

$$
\begin{aligned}
& M\left(P^{\prime} \Delta_{1}^{\prime} \Delta_{(1)}^{\prime} \lambda_{1}^{\prime} \lambda_{2}^{\prime} \lambda_{3}^{\prime} ; P \Delta_{2} \Delta_{(2)} \lambda_{1} \lambda_{2} \lambda_{3}\right) \\
& =\delta^{4}\left(P^{\prime}-P\right) \sum_{\lambda^{\prime}} \int d^{4} \Delta_{1}^{\prime \prime} d^{4} \Delta_{(i)}^{\prime} \frac{1}{P_{3}^{\prime}{ }^{2}-m_{3}{ }^{2}+i \eta}
\end{aligned}
$$

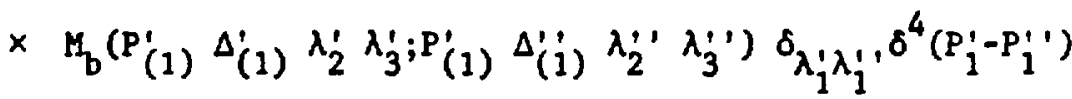

$$
\begin{aligned}
& \times M_{a}\left(P_{(2)} \Delta_{(2)}^{\prime} \lambda_{3}^{\prime} \lambda_{1}^{\prime \prime} ; P_{(2)} \Delta_{(2)} \lambda_{3} \lambda_{1}\right) \delta_{\lambda_{2}^{\prime}{ }^{\prime} \lambda_{2} \delta^{4}\left(P_{2}^{\prime \prime}-P_{2}\right)}
\end{aligned}
$$

Then, since $P^{\prime}=P$ and $P_{0}>|\vec{P}|$, there exists a real Lorentz transformation to the $O A C M$; and, since $\left(K_{(1)}^{\prime}\right)_{0}>\left|\vec{K}_{(1)}^{\prime}\right|$ and $\left(K_{(2)}\right)_{0}$ $>\left|\vec{R}_{2}\right|$, each of the two-body $M$ functions can be rewritten in terms of a CM M function. This implies that

$$
\begin{aligned}
M\left(P^{\prime}\right. & \left.\Delta_{1}^{\prime} \Delta_{(1)}^{\prime} \lambda_{1}^{\prime} \lambda_{2}^{\prime} \lambda_{3}^{\prime} ; P \Delta_{2} \Delta_{(2)} \lambda_{1} \lambda_{2} \lambda_{3}\right) \\
= & \delta^{4}\left(P^{\prime}-P\right) \sum_{v^{\prime} v^{\prime}} D_{\lambda_{1}^{\prime} v_{1}^{\prime}}^{s_{1}}(C(P)) D_{\lambda_{2}^{\prime} v_{2}^{\prime}}^{s_{2}}\left(C(P) C\left(K_{(1)}^{\prime}\right)\right) \\
& \times D_{\lambda_{3}^{\prime} v_{3}^{\prime}}^{s_{3}}\left(C(P) C\left(K_{(1)}^{\prime}\right)\right) M\left(P_{i} ; q_{1}^{\prime}\left(P_{1}^{\prime} v_{2}^{\prime} v_{3}^{\prime}\right) v_{1}^{\prime} ; q_{2}\left(P_{2} v_{3} v_{1}\right) v_{2}\right) \\
& \times D_{v_{1} \lambda_{1}}^{s_{1}}\left(C\left(K_{(2)}\right)^{-1} C(P)^{-1}\right) D_{v_{2} \lambda_{2}}^{s_{2}}\left(C(P)^{-1}\right) D_{v_{3} \lambda_{3}}^{s_{3}}\left(C\left(K_{(2)}\right)^{-1} C(P)^{-1}\right)
\end{aligned}
$$


where

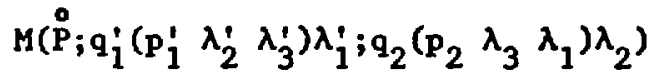

$$
\begin{aligned}
& =\sum_{\mu^{\prime} \cdot \lambda^{\prime}} \int d^{4} q_{1}^{\prime} d^{4} p_{1}^{\prime} \frac{1}{k_{3}^{\prime \prime}{ }^{2}-m_{3}{ }^{2}+i \eta} \\
& \times \mathcal{H}_{b}\left(\stackrel{\circ}{K^{\prime}}(1) ; P_{1}^{\prime} \lambda_{2}^{\prime} \lambda_{3}^{\prime} ; P_{1}^{\prime \prime} \mu_{2}^{\prime \prime} \mu_{3}^{\prime \prime}\right) \delta_{\lambda_{1}^{\prime} \mu_{1}^{\prime}} \delta^{4}\left(K_{1}^{\prime}-K_{1}^{\prime}\right) \\
& \times D_{\mu_{2}^{\prime} \lambda_{2}^{\prime \prime}}^{s_{2}^{\prime}}\left(C\left(K_{(1)}^{\prime}\right)^{-1}\right) D_{\mu_{3}^{\prime} \lambda_{3}^{\prime \prime}}^{s_{3}}\left(C\left(K_{(1)}^{\prime}\right)^{-1} c\left(K_{(2)}\right)\right) D_{\mu_{1}^{\prime} \lambda_{1}^{\prime}{ }^{\prime}\left(C\left(K_{(2)}\right)\right)} \\
& \times M_{a}\left(\stackrel{\circ}{K}_{(2)} ; P_{2}^{\prime \prime} \lambda_{3}^{\prime \prime} \lambda_{1}^{\prime \prime} ; p_{2} \lambda_{3} \lambda_{1}\right) \delta_{\lambda_{2}^{\prime} \lambda_{2}} \delta^{4}\left(K_{2}^{\prime \prime}-K_{2}\right)
\end{aligned}
$$

Finally, this $M$ function can be expressed in terms of the angular-momentum representations of the two-body functions, and the resultant $M$ function can be expanded in a total-angular-momentum representation. The equation for the $M$ function in that representation is 


$$
\begin{aligned}
& \mathbb{M}^{J}\left\{W_{i}\left(q_{i}^{\prime}\right)_{0} q_{i}^{\prime}\left(\left(p_{1}^{\prime}\right)_{0} p_{1}^{\prime} j_{i}^{\prime} m_{i}^{\prime} \lambda_{2}^{\prime} \lambda_{3}^{\prime}\right) \lambda_{i}^{\prime} ;\right. \\
& \left.\left(q_{2}\right)_{0} q_{2}\left(\left(p_{2}\right)_{0} p_{2} j_{2} m_{2} \lambda_{3} \lambda_{1}\right) \lambda_{2}\right\} \\
& =\underset{\lambda^{\prime} \mu^{\prime}{ }^{\prime}}{\sum} \int d\left(q_{1}^{\prime}\right)_{0} d\left(p_{1}^{\prime \prime}\right)_{0} p_{1}^{\prime \prime}{ }^{2} d p_{1}^{\prime} d\left(\cos \theta_{1}^{\prime}\right) \frac{1}{k_{3}^{\prime}{ }^{2}-m_{3}{ }^{2}+i \eta} \\
& \times M_{b}^{j}{ }^{j}\left(w_{(1)}^{\prime} ;\left(p_{1}^{\prime}\right)_{0} p_{1}^{\prime} \lambda_{2}^{\prime} \lambda_{3}^{\prime} ;\left(p_{1}^{\prime \prime}\right)_{0} p_{1}^{\prime \prime} \mu_{2}^{\prime \prime} \mu_{3}^{\prime \prime}\right) \\
& \times \tilde{c}_{j}\left\{W_{j} ;\left(q_{1}^{\prime}\right)_{0} q_{1}^{\prime}\left(\left(p_{1}^{\prime \prime}\right)_{0} p_{1}^{\prime \prime} j_{j}^{\prime} m_{1}^{\prime} \mu_{2}^{\prime \prime} \mu_{3}^{\prime}\right) \lambda_{j}^{\prime} ;\right. \\
& \left.\left(q_{2}^{\prime \prime}\right)_{0} q_{2}^{\prime \prime}\left(\left(p_{2}^{\prime \prime}\right)_{0} p_{2}^{\prime \prime} j_{2} m_{2} \lambda_{3}^{\prime \prime} \lambda_{1}^{\prime \prime}\right) \lambda_{2}\right\}
\end{aligned}
$$

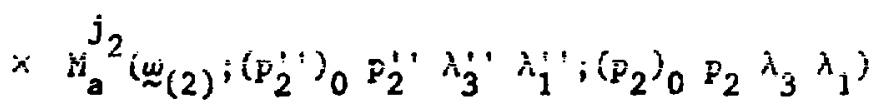

$$
\begin{aligned}
& \times \delta\left(\left(K_{1}^{\prime}\right)_{0}-\left(K_{1}^{\prime}\right)_{0}\right) \delta\left(\left(K_{2}^{\prime}\right)_{0}-\left(K_{2}\right)_{0}\right) \frac{1}{q_{2}{ }^{2}} \delta\left(q_{2}^{\prime}{ }^{\prime}-q_{2}\right)
\end{aligned}
$$

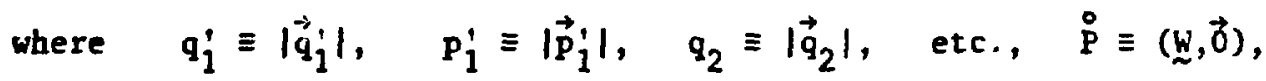

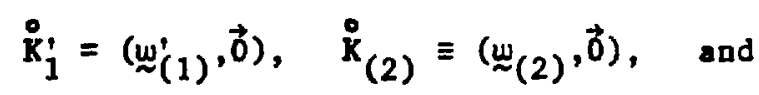




$$
\begin{aligned}
& \tilde{c}_{\mathrm{J}}\left\{w_{\sim} ;\left(q_{1}\right)_{0} q_{1}\left(\left(p_{1}\right)_{0} p_{1} j_{1} m_{1} \lambda_{2}^{\prime} \lambda_{3}^{\prime}\right) \lambda_{1}^{\prime} ;\right. \\
& \left.\left(q_{2}\right)_{0} q_{2}\left(\left(p_{2}\right)_{0} p_{2} j_{2} m_{2} \lambda_{3} \lambda_{1}\right) \lambda_{2}\right) \\
& \equiv\left(\frac{1}{4 \pi}\right)^{2}\left(\left(2 j_{1}+1\right)\left(2 j_{2}+1\right)\right)^{\frac{1}{2}} e^{i \pi s_{3}-i \pi s_{2}} \\
& \times \int d^{2} \hat{q}_{1} d \phi_{1} D_{m_{2}-\lambda_{2}, m_{1}-\lambda_{1}^{J}}^{J}\left(R\left(\hat{q}_{2}\right)^{-1} R\left(\hat{q}_{1}\right)\right) D_{m_{1}, \lambda_{2}^{\prime}-\lambda_{3}^{\dagger}}^{j_{1}}\left(R\left(\hat{p}_{1}\right)\right) \\
& \times D_{m_{2}, \lambda_{3}-\lambda_{1}}^{j_{2}}\left(R\left(\hat{p}_{2}\right)\right)^{*} D_{\lambda_{2}^{\prime} \lambda_{2}}^{s}\left(R\left(\hat{p}_{1}\right)^{-1} C\left(K_{(1)}\right)^{-1} R\left(\hat{q}_{2}\right) R(\pi, \pi)\right) \\
& \times D_{\lambda_{3} \lambda_{3}}^{s_{3}}\left(R(\pi, \pi)^{-1} R\left(\hat{p}_{1}\right)^{-1} C\left(K_{(i)}\right)^{-1} C\left(K_{(2)}\right) R\left(\hat{n}_{2}\right)\right) \\
& \times D_{\lambda_{1}^{\prime} \lambda_{1}}^{\delta_{1}}\left(R(\pi, \pi)^{-1} R\left(\hat{q}_{1}\right)^{-1} C\left(K{ }_{(2)}\right) R\left(\hat{p}_{2}\right) R(\pi, \pi)\right)
\end{aligned}
$$

The integral over $\hat{p}_{1}\left(d^{2} \hat{p}_{1} \equiv d \phi_{1} d\left(\cos \theta_{1}\right)\right)$ has been seperated such that the integral over $\phi_{1}$ is included in the definition of $\tilde{\mathrm{c}}_{\mathrm{J}}$. The reason for this will be clear after an explicit equation for $\tilde{C}_{J}$ bas been derived.

To obtain this equation, it is convenient to rewrite Eq. $6-29$ as 


$$
\begin{aligned}
& \tilde{\mathcal{C}}_{J}\left\{W_{i} ;\left(q_{1}\right)_{0} q_{1}\left(\left(p_{1}\right)_{0} p_{1} j_{1} m_{1} \lambda_{2}^{\prime} \lambda_{3}^{\prime}\right) \lambda_{1}^{\prime} ;\right. \\
& \left.\left(q_{2}\right)_{0} q_{2}\left(\left(p_{2}\right)_{0} p_{2} j_{2} m_{2} \lambda_{3} \lambda_{1}\right) \lambda_{2}\right\} \\
& =\left(\frac{1}{4 \pi}\right)^{2}\left(\left(2 j_{1}+1\right)\left(2 j_{2}+1\right)\right)^{\frac{2}{2}} e^{i \pi s_{3}-i \pi s_{2}} \\
& \times \int d \Phi_{1} d\left(\cos \underline{\theta}_{1}\right) d \phi_{1} D_{m_{2}-\lambda_{2}, m_{1}-\lambda_{1}^{\prime}}^{J}\left(R_{2}^{-1} R_{1}\right) d_{m_{1}, \lambda_{2}^{\prime}-\lambda_{3}^{j}\left(\theta_{1}\right)}^{j_{1}} \\
& \times d_{m_{2}, \lambda_{3}-\lambda_{1}}^{j_{2}}\left(\theta_{2}\right) D_{\lambda_{2} \lambda_{2}}^{s_{2}}\left(R\left(\theta_{1}, \hat{y}\right)^{-1} z\left(x_{(1)}\right)^{-1} R_{1}{ }^{-1} R_{2} R(\pi, \pi)\right) \\
& \times D_{\lambda_{3}^{1} \lambda_{3}}^{s_{3}}\left(R(\pi, \pi)^{-1} R_{R}\left(\theta_{1}, \hat{y}\right)^{-1} Z\left(x_{(1)}\right)^{-1_{R_{1}}}-1_{R_{2}} Z\left(x_{(2)}\right) R\left(\theta_{2}, \hat{y}\right)\right) \\
& \times D_{\lambda_{1}^{\prime} \lambda_{1}}^{s_{1}}\left(R(\pi, \pi)^{-1} R_{1}^{-1} R_{2} Z\left(X_{(2)}\right) R\left(\theta_{2}, \hat{y}\right) R(\pi, \pi)\right)
\end{aligned}
$$

where $x_{(1)} \equiv x_{K_{(1)}}, x_{(2)} \equiv x_{K_{(2)}}, d^{2} \hat{q}_{1} \equiv d \Phi_{1} d\left(\cos \Theta_{-1}\right)$,

and $\quad R_{2}^{-1} R_{1} \equiv R\left(\Phi_{2}, \underline{\theta}_{2}, \Phi_{2}\right)^{-1} R\left(\Phi_{1}, \underline{\theta}_{1}, \phi_{1}\right)$

$[R(\alpha, \beta, \gamma) \equiv R(\alpha, \hat{z}) R(\beta, \hat{y}) R(\gamma, \hat{z})]$.

The rotation $R_{2}^{-1} R_{1}$ will be evaluated in the next subsection, and it will be shown that it is equal to a rotation about the $\hat{\mathbf{y}}$ axis. It will also be shown that the entire integrand in Eq. $6-30$ is independent of the angles $\Phi_{1}, \underline{\theta}_{1}$, and $\phi_{1}$; hence, these integrations can be performed yielding an integration free equation for $\tilde{\mathrm{C}}_{\mathrm{J}}$. In 56.4 , Eq. 6-28 will be written in terms of $T$ functions. In that 
representation, the off-wass-shell recoupling coefficient can be simply related to the on-shell recoupling coefficient that is defined in Eq. 6-22.

\subsubsection{More Three-Body Kinematics}

To evaluate the rotation $R_{2}{ }^{-1} R_{1}$ in $E q .6-30$, consider the four-momentum of particle 1 in the $\mathrm{OACH}$ reference frame.

$$
\begin{aligned}
K_{1} & =r\left(\Phi_{1}, \underline{\theta}_{1}\right) r(\pi, \pi) K_{1}^{(z)} \\
& =r\left(\Phi_{2}, \underline{\theta}_{2}\right) z\left(X_{(2)}\right) r\left(\Phi_{2}, \theta_{2}\right) r(\pi, \pi) k_{1(2)}^{(z)}
\end{aligned}
$$

where $\quad \cosh x_{(i)}=\frac{\left(K_{(i)}\right)_{0}}{\underset{\sim}{w_{(i)}}}, \quad \sinh x_{(i)}=\frac{\left|\vec{q}_{i}\right|}{\underset{\sim}{w_{(i)}}}$,

${\underset{\sim}{(i)}}^{2}=k_{(i)}{ }^{2}=\left(k_{(i)}\right)_{0}^{2}-\left|\vec{q}_{i}\right|^{2}, \quad k_{1}^{(2)}=\left(\left(k_{1}\right)_{0}, 0,0,\left|\vec{q}_{1}\right|\right)$,

and $\mathrm{k}_{1(2)}^{(z)}=\left(\left(\mathrm{k}_{1(2)}\right)_{0}, 0,0,\left|\overrightarrow{\mathrm{p}}_{2}\right|\right)$

This implies that

$$
r_{1} r(\pi, \pi) k_{1}^{(z)}=r_{2} z\left(x_{(2)}\right) r\left(\pi, \pi-\theta_{2}\right) k_{1(2)}^{(z)}
$$


which can be rewritten by using the identity

$$
z(x) r(\theta, \hat{y})\left(\begin{array}{l}
k_{0} \\
0 \\
0 \\
k
\end{array}\right) \equiv\left(\begin{array}{c}
k_{0} \cosh x+k \sin x \cos \theta \\
k \sin \theta \\
0 \\
k_{0} \sinh x+k \cosh x \cos \theta
\end{array}\right)
$$

to obtain

$$
r_{1} r(\pi, \pi) k_{1}^{(2)}=r_{2} r\left(\pi, \xi_{1(2)}\right) k_{1}^{(2)}
$$

where $\quad z\left(\chi_{(2)}\right) r\left(\pi-\theta_{2}, \hat{y}\right) k_{1(2)}^{(z)} \equiv r\left(\xi_{1(2)}, \hat{y}\right) k_{1}^{(z)}$

with

$$
\begin{aligned}
& \left.\left(\mathrm{K}_{1}\right)_{0}=\left(k_{1(2)}\right)_{0} \cosh \chi_{(2)}-\left|\vec{p}_{2}\right| \sinh x_{(2)}\right)^{\cos \theta_{2}} \\
& \left|\vec{q}_{1}\right| \sin \xi_{1(2)}=\left|\vec{p}_{2}\right| \sin \theta_{2} \\
& \left|\vec{q}_{1}\right| \cos \xi_{1(2)}=\left(k_{1(2)}\right)_{0} \sinh \chi_{(2)}-\left|\vec{p}_{2}\right| \cosh x_{(2)} \cos \theta_{2}
\end{aligned}
$$

and $0 \leqq \xi_{1(2)} \leqq \pi$ because $0 \leqq \theta_{2} \leqq \pi$. Hence, the rotation $R_{2}^{-1} R_{1}$ is determined up to a possible rotation about the $\hat{z}$ axis.

$$
R_{2}^{-1} R_{1}=R\left(\pi-\xi_{1(2)}, \hat{y}\right) R\left(\zeta_{1(2)}, \hat{z}\right)
$$

These rotation are shown in Fig. 6.2. 


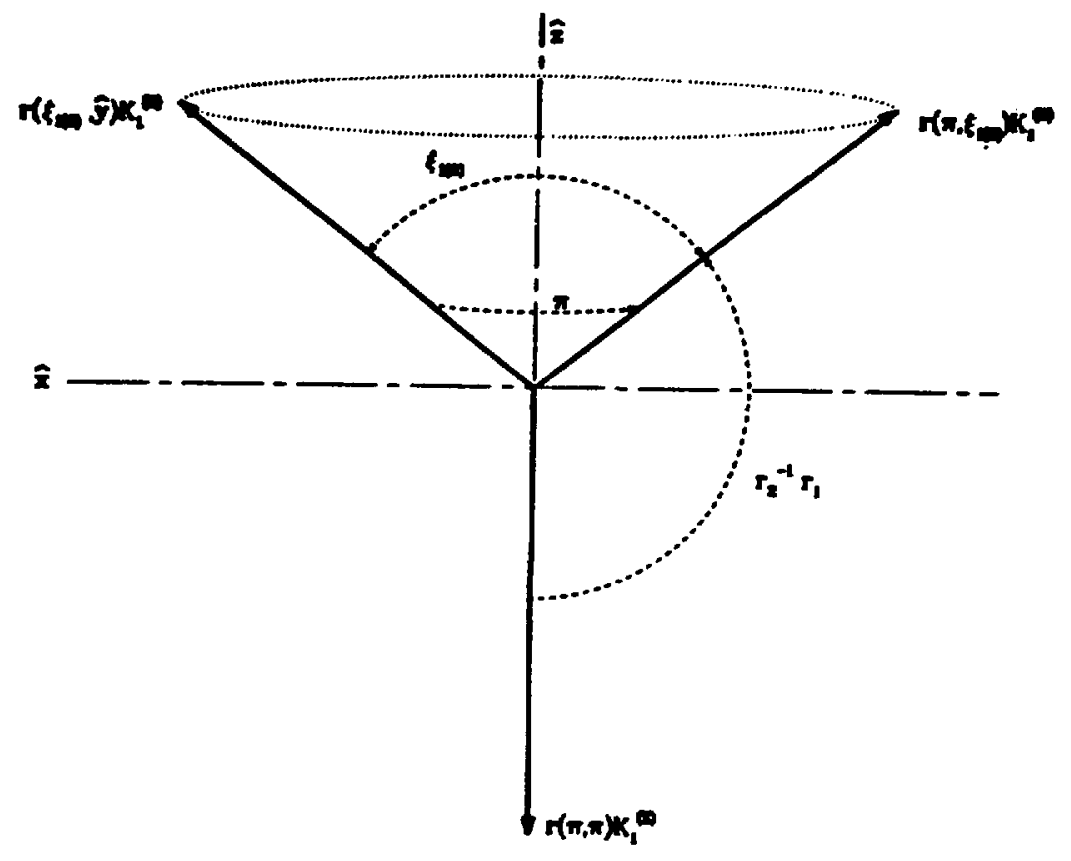

Fig. 6.2 The rotations of $K_{1}$.

The angle $\zeta_{1(2)}$ in Eq. 6-39 is determined by considering the four-monentum of particle 2 in the $\mathrm{OACM}$ reference frame.

$$
\begin{aligned}
\mathbf{K}_{2} & =r\left(\Phi_{2}, \underline{\theta}_{2}\right) r(\pi, \pi) K_{2}^{(z)} \\
& =r\left(\Phi_{1}, \underline{\theta}_{1}\right) z\left(x_{(1)}\right) r\left(\phi_{1}, \theta_{1}\right) k_{2(1)}^{(z)}
\end{aligned}
$$

This implies that

$$
\begin{aligned}
x_{1}^{-1} x_{2} r(\pi, \pi) x_{2}^{(z)} & =r\left(\xi_{2(1)}, \hat{y}\right) x_{2}^{(z)} \\
& =r\left(\xi_{1(2)}, \hat{z}\right)^{-1} r\left(\pi-\xi_{1(2)}, \hat{y}\right)^{-1} r(\pi, \pi) x_{2}^{(z)} \quad(6-41)
\end{aligned}
$$


where $\quad z\left(x_{(1)}\right) r\left(\theta_{1}, \hat{y}\right) k_{2(1)}^{(z)} \equiv r\left(\xi_{2(1)}, \hat{y}\right) x_{2}^{(z)}$

with $\quad\left(k_{2}\right)_{0}=\left(k_{2(1)}\right)_{0} \cosh x_{(1)}+\left|\vec{p}_{1}\right| \sinh x_{(1)} \cos \theta_{1}$

$$
\begin{aligned}
& \left|\vec{q}_{2}\right| \sin \xi_{2(1)}=\left|\vec{p}_{1}\right| \sin \theta_{1} \\
& \left|\vec{q}_{2}\right| \cos \xi_{2(1)}=\left(k_{2}(1)\right)_{0} \sinh \chi_{(1)}+\left|\vec{p}_{1}\right| \cosh \chi_{(1)} \cos \theta_{1}
\end{aligned}
$$

and $0 \leqq \xi_{2(1)} \leqq \pi$. These rotations are shown in Fig. 6.3.

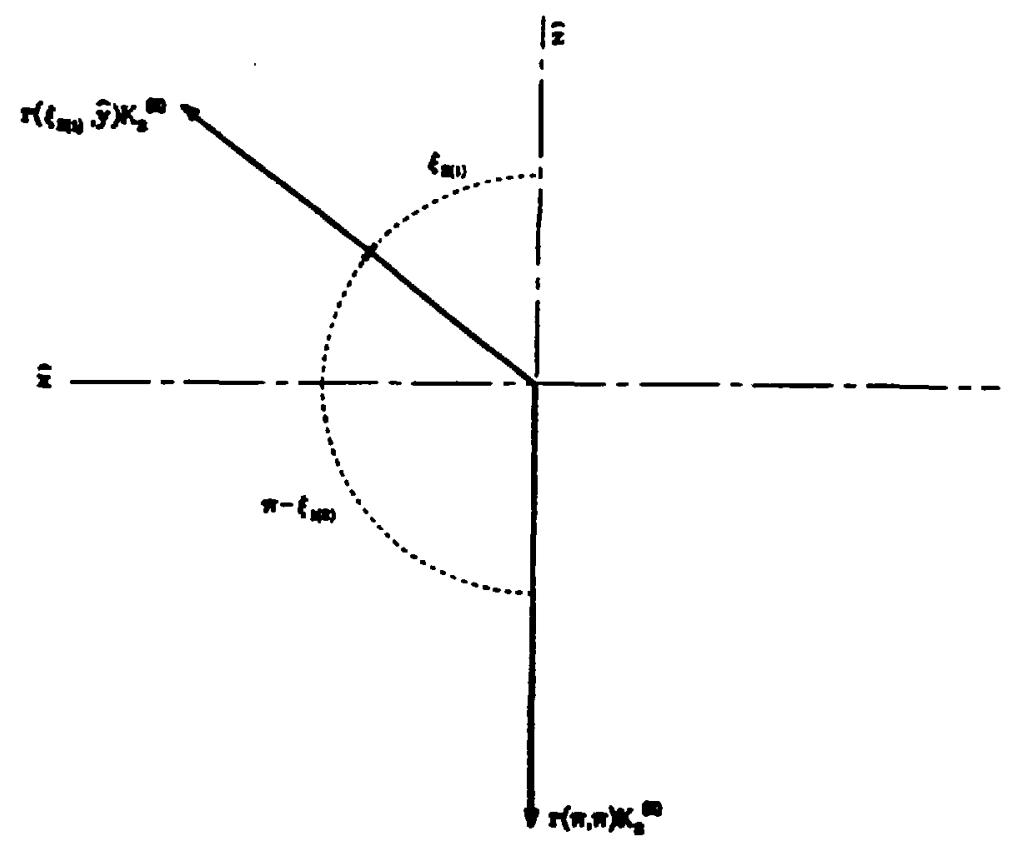

Fig. 6.3 The rotations of $\mathrm{K}_{2}$. 
Thus, $\xi_{1(2)}$ equals either 0 or $2 \pi$ and $\xi_{2(1)}=\xi_{1(2)}$. The particular value of $\zeta_{1(2)}$ is not important because either $\zeta_{1(2)}=0$ or $\zeta_{1(2)}=2 \pi$ will yield the same recoupling coefficient (Eq. 6-30); hence, the replacement

$$
R_{2}^{-1} R_{1} \rightarrow R\left(\pi-\xi_{1(2)}, \hat{y}\right)
$$

and $\quad R_{1}^{-1} R_{2} \rightarrow R\left(\xi_{1(2)}-\pi, \hat{y}\right)$

can be made in the equation for that coefficient.

Eqs. 6-36 through 6-38 and Eqs. 6-43 through 6-45 imply that the angles $\theta_{1}$ and $\theta_{2}$ are completely determined by the energy and momentium variables. In particular,

$$
\cos \theta_{2}=\frac{1}{\left|\vec{p}_{2}\right|\left|\vec{q}_{2}\right|}\left(\left(k_{1(2)}\right)_{0}\left(K_{(2)}\right)_{0}-{\underset{\sim}{w}(2)}_{\left.\left(K_{1}\right)_{0}\right)}\right.
$$

and $\quad \cos \theta_{1}=\frac{1}{\left|\vec{p}_{1}\right|\left|\vec{q}_{1}\right|}\left(\underline{w}_{(1)}\left(K_{2}\right)_{0}-\left(k_{2(1)}\right)_{0}\left(K_{(1)}\right)_{0}\right)$

where the energies are determined from the definitions of the relative momenta (Eqs. 6-8 and 6-9).

$$
\begin{aligned}
& \left(K_{(i)}\right)_{0}=\left(q_{i}\right)_{0}+\frac{1}{2}\left(1+\frac{1}{s}\left(m_{(i)}{ }^{2}+m_{i}{ }^{2}\right)\right) \underset{\sim}{W} \\
& \left(k_{i}\right)_{0}=-\left(q_{i}\right)_{0}+\frac{1}{2}\left(1-\frac{1}{s}\left(m_{(i)}{ }^{2}+m_{i}{ }^{2}\right)\right) \underset{\sim}{W}
\end{aligned}
$$




$$
\begin{aligned}
& \left(k_{1(2)}\right)_{0}=-\left(p_{2}\right)_{0}+\frac{1}{2}\left(1-\frac{1}{s_{2}}\left(m_{3}^{2}-m_{1}^{2}\right)\right)_{w_{(2)}} \\
& \left.\left(k_{2(1)}\right)_{0}=\left(p_{1}\right)_{0}+\frac{1}{2}\left(1+\frac{1}{s_{1}}\left(m_{2}{ }^{2}-m_{3}^{2}\right)\right)\right)_{(1)}
\end{aligned}
$$

with $\quad s \equiv \underline{w}^{2}, \quad s_{i} \equiv{\underset{\sim}{w}}_{(i)}{ }^{2}, \quad{\underset{\sim}{w}}_{(i)} \equiv\left(\left(K_{(i)}\right)_{0}{ }^{2}-\left|\vec{q}_{i}\right|^{2}\right)^{\frac{1}{2}}$,

and $m_{(i)} \equiv\left(\left|\Delta(i){ }^{2}\right|+m_{j}{ }^{2}\right)^{\frac{1}{2}}+\left(\left|\Delta(i){ }^{2}\right|+m_{k}{ }^{2}\right)^{\frac{1}{2}}$

where $\quad \Delta_{(i)}{ }^{2}=\left(p_{i}\right)_{0}^{2}-\left|\vec{p}_{i}\right|^{2}$.

(Note: In the on-shell limit these variables are $\left(K_{(i)}\right)_{0}=w_{(i)}$, $\left(k_{i}\right)_{0}=w_{i}$, and $\left.\left(k_{i(j)}\right)_{0}=w_{i(j)} \cdot\right)$

An equivalent procedure can be followed to obtain relations between the energies and angles for particle 3 . The four-momentum of this particle in the OACM is

$$
\begin{aligned}
x_{3} & =r\left(\Phi_{2}, \underline{\theta}_{2}\right) z\left(x_{(2)}\right) r\left(\phi_{2}, \theta_{2}\right) k_{3(2)}^{(z)} \\
& =r\left(\Phi_{1}, \underline{\theta}_{1}\right) z\left(X_{(1)}\right) r\left(\phi_{1}, \theta_{1}\right) r(\pi, \pi) k_{3(1)}^{(z)}
\end{aligned}
$$

which implies that

$$
r_{2} r\left(\xi_{3(2)}, \hat{y}\right) k_{3}^{(2)}=r_{1} r\left(\pi, \xi_{3(1)}\right) k_{3}^{(2)}
$$


where $z\left(x_{(2)}^{x}\right) r\left(\theta_{2}, \hat{y}\right) k_{3(2)}^{(z)} \equiv r\left(\xi_{3(2)}, \hat{y}\right) k_{3}^{(z)}$
and $z\left(x_{(1)}\right) r\left(\pi-\theta_{1}, \hat{y}\right) k_{3(1)}^{(z)} \equiv r\left(\xi_{3(1)}, \hat{y}\right) k_{3}^{(z)}$

with $\left(k_{3}\right)_{0}=\left(k_{3(2)}\right)_{0} \cosh \chi_{(2)}+\vec{p}_{2} \mid \sinh \chi_{(2)} \cos \theta_{2}$

$$
\left|\vec{q}_{3}\right| \sin \xi_{3(2)}=\left|\vec{p}_{2}\right| \sin \theta_{2}
$$

$$
\left|\vec{q}_{3}\right| \cos \xi_{3(2)}=\left(k_{3(2)}\right)_{0} \sinh \chi_{(2)}+\left|\vec{p}_{2}\right| \cosh \chi_{(2)} \cos \theta_{2}
$$

and

$$
\begin{aligned}
& \left(\mathrm{K}_{3}\right)_{0}=\left(\mathrm{k}_{3(1)}\right)_{0} \cosh x_{(1)}-\left|\overrightarrow{\mathrm{p}}_{1}\right| \sinh x_{(1)} \cos \theta_{1} \\
& \left|\overrightarrow{\mathrm{q}}_{3}\right| \sin \xi_{3(1)}=\left|\overrightarrow{\mathrm{p}}_{1}\right| \sin \theta_{1} \\
& \left|\overrightarrow{\mathrm{q}}_{3}\right| \cos \xi_{3(1)}=\left(\mathrm{k}_{3(1)}\right)_{0} \sinh \chi_{(1)}-\overrightarrow{\mathrm{p}}_{1} \mid \cosh x_{(1)} \cos \theta_{1}
\end{aligned}
$$

Hence, $\quad r\left(\xi_{1(2)}+\xi_{3(2)}-\pi, \hat{y}\right) k_{3}^{(z)}=r\left(\pi, \xi_{3(1)}\right) k_{3}^{(z)}$

and $\xi_{1(2)}=\pi-\xi_{3(1)}-\xi_{3(2)}$.

These rotations are shown in Fig. 6.4. 


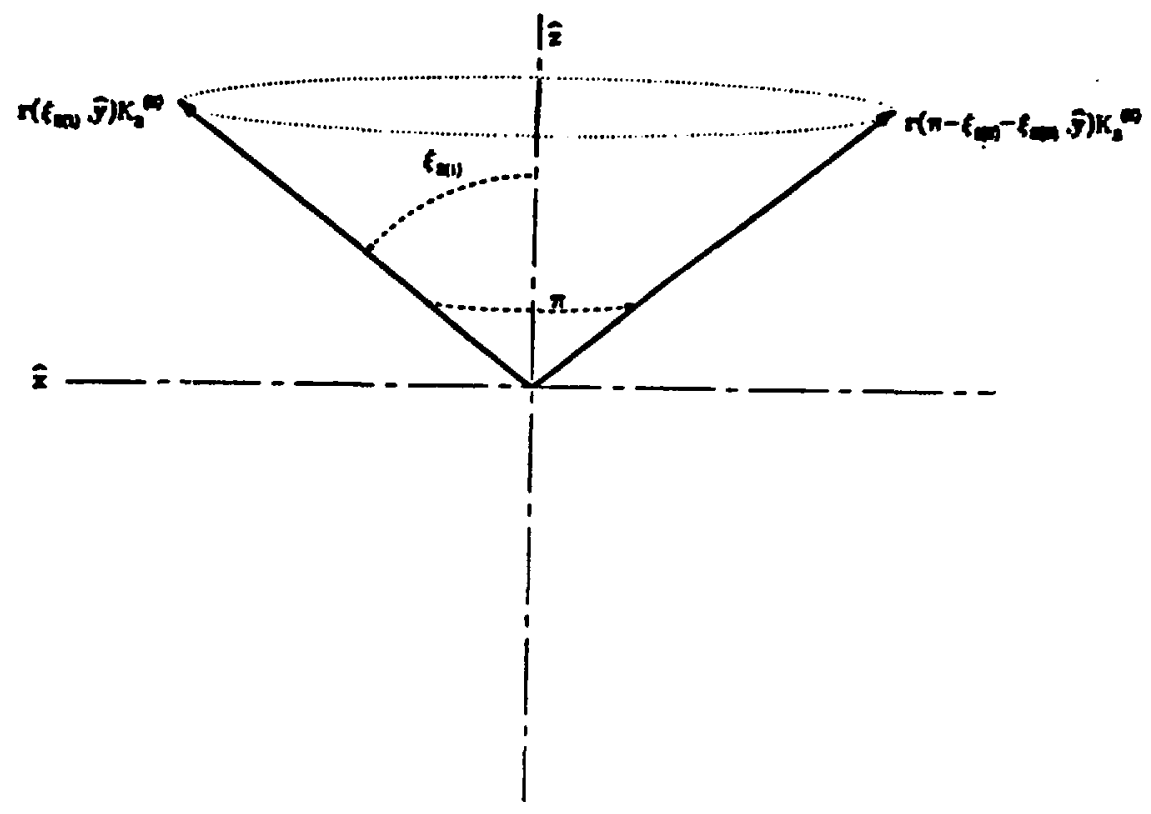

Fig. 6.4 The rotations of $\mathrm{K}_{3}$.

\subsubsection{Return to the M Function Recoupling Coefficient}

The results of $\$ 6.3 .1$ can now be inserted into the definition of the off-mass-shell $M$ function recoupling coefficient (Eq. 6-30), and the integrations over $\Phi_{1}, \underline{\theta}_{1}$, and $\phi_{1}$ can be done to obtain 


$$
\begin{aligned}
& \text {, } \tilde{c}_{J}\left\{W ;\left(q_{1}\right)_{0} q_{1}\left(\left(p_{1}\right)_{0} p_{1} j_{1} m_{1} \lambda_{2}^{\prime} \lambda_{3}^{\prime}\right) \lambda_{1} ;\right. \\
& \left.\left(q_{2}\right)_{0} q_{2}\left(\left(p_{2}\right)_{0} \quad p_{2} j_{2} m_{2} \lambda_{3} \lambda_{1}\right) \lambda_{2}\right\} \\
& =\frac{1}{2}\left(\left(2 j_{1}+1\right)\left(2 j_{2}+1\right)\right)^{\frac{1}{2}} e^{i \pi s_{3}}(-1)^{s_{2}-\lambda_{2}} \\
& \times d_{m_{2}-\lambda_{2}, m_{1}-\lambda_{1}^{\prime}}^{J}\left(\pi-\xi_{1(2)}\right) d_{m_{1}, \lambda_{2}^{\prime}-\lambda_{3}^{\prime}}^{j_{1}}\left(\theta_{1}\right) d_{m_{2}, \lambda_{3}-\lambda_{1}}^{j_{2}}\left(\theta_{2}\right) \\
& \times \mathrm{D}_{\lambda_{2}^{\prime} \lambda_{2}}^{\mathrm{s}_{2}}\left(R\left(\theta_{1}, \hat{\mathrm{y}}\right)^{-1} \mathrm{Z}\left(\mathrm{x}_{(1)}\right)^{-1} \mathrm{R}\left(\xi_{2(1)}, \hat{\mathrm{y}}\right)\right) \\
& \times \mathrm{D}_{\lambda_{1} \lambda_{1}}^{s_{1}}\left(R\left(\xi_{1(2)}, \hat{y}\right)^{-1} Z\left(\chi_{(2)}\right) R\left(\pi-\theta_{2}, \hat{y}\right)\right) \\
& \times D_{\lambda_{3}^{\prime} \lambda_{3}}^{s_{3}}\left(R\left(\pi-\theta_{1}, \hat{y}\right)^{-1} Z\left(X_{(1)}\right)^{-1} R\left(\xi_{3(1)}, \hat{y}\right)\right) \\
& \left.R(\pi, \hat{z})^{-1} R\left(\xi_{3(2)}, \hat{y}\right)^{-1} Z\left(x_{(2)}\right) R\left(\theta_{2}, \hat{y}\right)\right)
\end{aligned}
$$

Another consequence of the kinematic analysis in $\$ 6.3 .1$ is the ability to make a change of variables in the equation for the $M$ function (Eq. 6-28). This variable change is detailed in \$E-1 and results in the following equation for $\mathrm{M}^{\mathrm{J}}$ : 


$$
\begin{aligned}
& \boldsymbol{M}^{J}\left\{\underline{W}_{j}\left(q_{1}^{\prime}\right)_{0} q_{1}^{\prime}\left(\left(p_{1}^{\prime}\right)_{0} p_{1}^{\prime} j_{1}^{\prime} m_{1}^{\prime} \lambda_{2}^{\prime} \lambda_{3}^{\prime}\right) \lambda_{1}^{\prime} ;\right. \\
& \left.\left(q_{2}\right)_{0} q_{2}\left(\left(p_{2}\right)_{0} p_{2} j_{2} m_{2} \lambda_{3} \lambda_{1}\right) \lambda_{2}\right\} \\
& =\lambda^{\prime} \sum_{\mu^{\prime}} \int_{-1}^{1} d\left(\cos \xi_{2(1)}^{\prime}\right) \frac{1}{k_{3}^{\prime}{ }^{2}-m_{3}{ }^{2}+i \eta} \\
& \left.\times \mathrm{M}^{j}{ }^{\prime}{\underset{\sim}{(1)}}_{(1)}^{\prime} ;\left(\mathrm{p}_{1}^{\prime}\right)_{0} \mathrm{p}_{1}^{\prime} \lambda_{2}^{\prime} \lambda_{3}^{\prime} ;\left(\mathrm{p}_{1}^{\prime}\right)_{0} \mathrm{p}_{1}^{\prime \prime} \mu_{2}^{\prime \prime} \mu_{3}^{\prime \prime}\right) \\
& \times \tilde{c}_{\mathrm{J}}\left(\underline{W} ;\left(q_{1}^{\prime \prime}\right)_{0} q_{1}^{\prime}\left(\left(p_{1}^{\prime \prime}\right)_{0} p_{1}^{\prime \prime} j_{1}^{\prime} m_{1}^{\prime} \mu_{2}^{\prime \prime} \mu_{3}^{\prime \prime}\right) \lambda_{1}^{\prime} ;\right. \\
& \left.\left(q_{2}^{\prime \prime}\right)_{0} q_{2}\left(\left(p_{2}^{\prime \prime}\right)_{0} p_{2}^{\prime \prime} j_{2} m_{2} \lambda_{3}^{\prime \prime} \lambda_{1}^{\prime}\right) \lambda_{2}\right\} \\
& \left.\times M^{j} \stackrel{\sim}{w}_{(2)} ;\left(p_{2}^{\prime \prime}\right)_{0} p_{2}^{\prime \prime} \lambda_{3}^{\prime \prime} \lambda_{1}^{\prime \prime} ;\left(p_{2}\right)_{0} p_{2} \lambda_{3} \lambda_{1}\right)
\end{aligned}
$$

The energy and momentum variables that are labeled by a double prime in this equation can be determined from Eqs. 6-35 through 6-37 and Eqs. 6-43 through 6-45 (also see \$E-1).

\subsection{The T Function Recoupling Coefficient}

Because the $T$ function ( $\$ 5.3$ ) is intimately related to the reaction amplitude and because it has simple space-inversion properties, most computational methods will be developed in terms of the $T$ function instead of the $M$ function. The three-body $T$ function that corresponds to the $M$ function in Eq. 6-69 is (cf., Eq. 5-29) 


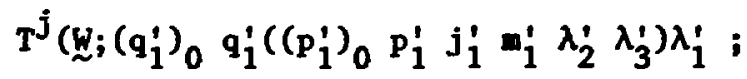

$$
\begin{aligned}
& \left.\left(q_{2}\right)_{0} q_{2}\left(\left(p_{2}\right)_{0} p_{2} j_{2} m_{2} \lambda_{3} \lambda_{1}\right) \lambda_{2}\right) \\
& =e^{-\frac{1}{2}\left(\lambda_{1}^{\prime} \bar{x}_{1}^{\prime}+\lambda_{2}^{\prime} \bar{x}_{2(1)}^{\prime}+\lambda_{3}^{+} \bar{x}_{3(1)}^{\prime}-\lambda_{2} \bar{x}_{2}-\lambda_{3} \bar{x}_{3(2)}-\lambda_{1} \bar{x}_{1(2)}\right)} \\
& \times M^{j}\left\{W_{i}\left(q_{1}^{\prime}\right)_{0} q_{1}^{\prime}\left(\left(p_{1}^{\prime}\right)_{0} p_{1}^{\prime} j_{1}^{\prime} m_{1}^{\prime} \lambda_{2}^{\prime} \lambda_{3}^{\prime}\right) \lambda_{1}^{\prime} ;\right. \\
& \left.\left(q_{2}\right)_{0} q_{2}\left(\left(p_{2}\right)_{0} p_{2} j_{2} m_{2} \lambda_{3} \lambda_{1}\right) \lambda_{2}\right\}
\end{aligned}
$$

where $\tanh \bar{x}_{2} \equiv \frac{\left|\overrightarrow{\mathrm{K}}_{2}\right|}{\varepsilon_{2}\left(\left|\overrightarrow{\mathrm{K}}_{2}\right|\right)}=\frac{\mathrm{q}_{2}}{\varepsilon_{2}\left(\mathrm{q}_{2}\right)}$

$$
\tanh \bar{x}_{1(2)} \equiv \frac{\left|\vec{p}_{2}\right|}{\varepsilon_{1}\left(\left|\vec{p}_{2}\right|\right)}=\frac{p_{2}}{w_{1(2)}}
$$

and so on. (Note: In this definition of the three-body $T$ function, the two particles that are coupled to form a two-body $\mathrm{CM}$ state are multiplied by $\exp \left( \pm \xi_{j} \bar{x}_{j(i)}\right)$ where $\bar{x}_{j(i)}$ is defined with respect to the two-body center-of-mass; whereas, the third particle is multiplied by $\exp \left( \pm \frac{k_{k}}{\lambda_{k}} \bar{x}_{k}\right)$ where $\bar{x}_{k}$ is defined with respect to the threebody center-of-mass. The reason for this can be seen by inspection of Eqs. 6-26 or 6-73.)

This $T$ function satisfies on equation that is identical to Eq. 6-69, except that within that equation the two-body $M$ functions are replaced by their corresponding $T$ functions and the recoupling 
coefficient $\tilde{\mathrm{C}}_{\mathrm{J}}$ is replaced by the following "off-mass-shell $\mathrm{T}$ function recoupling coefficient":

$$
\begin{aligned}
& C_{J}\left\{W ;\left(q_{1}\right)_{0} q_{1}\left(\left(p_{1}\right)_{0} p_{1} j_{1} m_{1} \lambda_{2}^{\prime} \lambda_{3}^{\prime}\right) \lambda_{1}^{\prime} ;\right. \\
& \left.\left(q_{2}\right)_{0} q_{2}\left(\left(p_{2}\right)_{0} p_{2} j_{2} m_{2} \lambda_{3} \lambda_{1}\right) \lambda_{2}\right\} \\
& =\frac{1}{2}\left(\left(2 j_{1}+1\right)\left(2 j_{2}+1\right)\right)^{\frac{1}{2}} e^{i \pi s_{3}}(-1)^{s_{2}-\lambda_{2}} \\
& \times d_{m_{2}-\lambda_{2}, m_{1}-\lambda_{1}^{\prime}}^{J}\left(\pi-\xi_{1(2)}\right) d_{m_{1}, \lambda_{2}^{\prime}-\lambda_{3}^{\prime}}^{j_{1}}\left(\theta_{1}\right) d_{m_{2}, \lambda_{3}-\lambda_{1}}^{j_{2}}\left(\theta_{2}\right) \\
& \times D_{\lambda_{2}^{+} \lambda_{2}}^{\delta_{2}}\left(Z\left(\bar{x}_{2(1)}\right)^{-1} R\left(\theta_{1}, \hat{y}\right)^{-1} Z\left(x_{(1)}\right)^{-1} R\left(\xi_{2(1)}, \hat{y}\right) Z\left(\bar{x}_{2}\right)\right) \\
& \times D_{\lambda_{1} \lambda_{1}}^{s_{1}}\left(Z\left(\bar{x}_{1}\right)^{-1} R\left(\xi_{1(2)}, \hat{y}\right)^{-1} z\left(x_{(2)}\right) R\left(\pi-\theta_{2}, \hat{y}\right) Z\left(x_{1(2)}\right)\right) \\
& \times D_{\lambda_{3}^{\prime} \lambda_{3}}^{s_{3}}\left(z\left(\bar{x}_{3(1)}\right)^{-1} R\left(\pi-\theta_{1}, \hat{y}\right)^{-1} z\left(x_{(1)}\right)^{-1}\right. \\
& \left.\times R(\pi, \hat{z})^{-1} R\left(\xi_{3(2)}, \hat{y}\right) Z\left(x_{(2)}\right) R\left(\theta_{2}, \hat{y}\right) Z\left(\bar{x}_{3(2)}\right)\right)
\end{aligned}
$$

where the identity $D_{\lambda^{\prime} \lambda}^{s}(2(x)) \equiv \delta_{\lambda^{\prime} \lambda} e^{\lambda x / 2}$ has been used to incorporate the exponential factors into the rotation matrices.

The relation between this recoupling coefficient and the on-shell recoupling coefficient that is shown in Eq. 6-22 can now be determined by using the following identity (cf., Eqs. 2-30, 2-33 and 2-34): 


$$
Z\left(X_{(i)}\right) R(\theta, \hat{y}) Z\left(\bar{x}_{j(i)}\right)=R\left(\xi_{j(i)}, \hat{y}\right) Z\left(x_{j}\right) R\left(\alpha_{j(i)}, \hat{y}\right)
$$

$$
\text { where } \begin{aligned}
\tan \xi_{j(i)} & =\frac{\sinh \bar{x}_{j(i)} \sin \theta}{\cosh \bar{x}_{j(i)} \sinh (i)+\sinh \bar{x}_{j(i)} \cosh (i) \cos \theta} \\
& =\frac{w_{(i)} p_{i} \sin \theta}{w_{j(i)} q_{i}+p_{i}\left(K_{(i)}\right)_{0} \cos \theta}
\end{aligned}
$$

$$
\begin{aligned}
\tan \alpha_{j(i)} & =\frac{\sinh \chi_{(i)} \sin \theta_{i}}{\sinh \bar{\chi}_{j(i)} \cosh \chi_{(i)}+\cosh \bar{\chi}_{j(i)} \sinh \chi_{(i)} \cos \theta} \\
& =\frac{m_{j} q_{i} \sin \theta}{p_{i}\left(\mathrm{~K}_{(i)}\right)_{0}+w_{j(i)} q_{i} \cos \theta}
\end{aligned}
$$

and $\cosh x_{j}=\cosh \chi_{(i)} \cosh \bar{x}_{j(i)}+\sinh \chi_{(i)} \sinh \bar{x}_{j(i)} \cos \theta$

$$
=\frac{1}{m_{j} w_{(i)}}\left(\left(K_{(i)}\right)_{0} w_{j(i)}+q_{i} p_{i} \cos \theta\right)
$$

to obtain

$$
\begin{aligned}
& Z\left(\bar{x}_{j}\right)^{-1} R\left(\xi_{j(i)}, \hat{y}\right)^{-1} Z\left(x_{(i)}\right) R(\theta, \hat{y}) Z\left(\bar{x}_{j(i)}\right) \\
& \quad=\left\{Z\left(\bar{x}_{j}\right)^{-1} R\left(\xi_{j(i)}-\xi_{j(i)}, \hat{y}\right) Z\left(x_{j}\right)\right\} R\left(\alpha_{j(i)}, \hat{y}\right)
\end{aligned}
$$

In the on-shell limit, the quantity in \{\} in this equation is equal to one and the on-sbell $T$ function recoupling coefficient is simply 


$$
\begin{aligned}
& c_{J}\left\{W ; q_{1}\left(p_{1} j_{1} \Phi_{1} \lambda_{2}^{\prime} \lambda_{3}^{\prime}\right) \lambda_{1}^{\prime} ; q_{2}\left(p_{2} j_{2} m_{2} \lambda_{3} \lambda_{1}\right) \lambda_{2}\right\} \\
& =\frac{1}{2}\left(\left(2 j_{1}+1\right)\left(2 j_{2}+1\right)\right)^{\frac{1}{2}}(-1)^{s_{3}+\lambda_{3}^{\prime}+s_{2}-\lambda_{2}} \\
& \left.\times d_{m_{2}-\lambda_{2}, m_{1}-\lambda_{1}^{j}}^{j}\left(\pi-\xi_{1(2)}\right) d_{m_{1}, \lambda_{2}^{\prime}-\lambda_{3}^{\prime}}^{j_{1}} \theta_{1}\right) d_{m_{2}, \lambda_{3}-\lambda_{1}}^{j_{2}}{ }^{\left(\theta_{2}\right)} \\
& \times d_{\lambda_{2}^{\prime} \lambda_{2}}^{s_{2}}{ }^{\left(-\alpha_{2(1)}\right)}{ }_{d_{\lambda}{ }_{1} \lambda_{1}}^{s_{1}}\left(\alpha_{1(2)}\right) d_{\lambda_{3}^{\prime} \lambda_{3}}^{s_{3}}\left(\alpha_{3(1)}+\alpha_{3(2)}\right) \quad \text { (6-79) }
\end{aligned}
$$

where, because the relative energies are zero in the on-shell limit, these labels have been suppressed. It is shown in $\$ \mathrm{E}-3$ that this is equal the recoupling coefficient that was defined in Eq. 6-22. (Note: The "energies" $w_{j(i)}$ in Eqs. 6-75 through 6-77 are the "on-shell energies" - i.e., $w_{j(i)} \equiv\left(p_{i}^{2}+m_{j}^{2}\right)^{\frac{1}{2}}$, etc.)

\subsection{Isospin}

In addition to its space-time properties, the singleparticle state can have an electric charge. The state-vector labels that correspond to this charge are called the "isospin" and are defined in analogy with the definition of spin in \$2.1. In particular, the single-particle state vector for a particle with total isospin $\tau$ and isospin projection $\mu$ is denoted by $|\tau \mu\rangle$, and this -tate-vector rotates in the isospin space as a $2 \tau+1$ component spinor. 


$$
U\left(R_{I}\right)|\tau \mu\rangle=\sum_{\mu^{\prime}} D_{\mu^{\prime} \mu^{\prime}}^{\tau}\left(R_{I}\right)\left|\tau \mu^{\prime}\right\rangle
$$

where $R_{I}$ is an element of $S U(2)_{\text {isospin }}$. The charge of the state with isospin projection $\mu$ is defined to be $Q \equiv \mu+\frac{1}{2} Y$, where $Y$ is the hypercharge of the single-particle state. The hypercharge is yet another property of the particle and will not be discussed here. (Note: $Y=1$ for the nucleon, which is an isospin $\frac{1}{2}$ particle; and $Y=0$ for the pion, which is an isospin 1 particle.)

For the two- and three-particle states it is a straightforward task to construct a basis of the multiple-particle isospin Hilbert space that rotates as a single-particle spinor. This is accomplished with Clebsch-Gordon coefficients as in \$3.3.1. The two particle state vector in the total-isospin basis is defined as

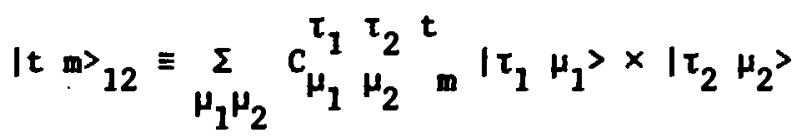

where the subscript 12 denotes the order in which the two particles have been coupled. The three-particle state vector in the totalisospin basis is defined as

$$
\text { IT M } \left.t_{3}\right\rangle \equiv \sum_{\mu_{3} m_{3}} c_{m_{3}}^{t_{3}} \tau_{3}^{\tau_{3} T} \mu_{3}\left|t_{3}=_{3}\right\rangle_{12} \times\left|\tau_{3} \mu_{3}\right\rangle
$$


It is evident that the order in which the particles appear in this state vector can be changed. This gives rise to the threebody isospin recoupling coefficient $j k^{\left\langle T^{\prime}\right.} M^{\prime} t_{i}\left|T M t_{k}\right\rangle_{i j}$, which is easily determined by manipulating the Clebsch-Gordon coefficients. The result is

$$
\begin{aligned}
j k\left\langle T^{\prime} M^{\prime} t_{i}\right. & \left|T M t_{k}\right\rangle_{i j}=\delta_{T^{\prime} T} \delta_{M^{\prime} M}(-1)^{\tau_{i}+t_{i}-T} \\
\times & \left(\left(2 t_{i}+1\right)\left(2 t_{k}+1\right)\right)^{\frac{3}{2}} W\left(\tau_{j} \tau_{k} \tau_{i} T ; t_{i} t_{k}\right)
\end{aligned}
$$

where $W\left(\tau{ }_{j}{ }_{k}{ }^{\tau}{ }_{i} T ; t_{i} t_{k}\right)$ is a Racah coefficient (Eq. A-?). Including the isospin labels the complete on-shell three-body recoupling coefficient is defined as

$$
\begin{aligned}
c_{J T}\left\{W ; q_{i}\left(p_{i} j_{i} m_{i} \lambda_{j}^{\prime} \lambda_{k}^{\prime}\right) \lambda_{i}^{\prime} t_{i} ; q_{k}\left(p_{k} j_{k} m_{k} \lambda_{i} \lambda_{j}\right) \lambda_{k} t_{k}\right\} \\
\equiv(-1){ }_{i}^{\tau_{i}+\tau_{i}-T}\left(\left(2 t_{i}+1\right)\left(2 t_{k}+1\right)\right)^{\frac{1}{2}} W\left(\tau_{j} \tau_{k} \tau_{i} T ; t_{i} t_{k}\right) \\
\quad \times c_{J}\left\{W_{j} q_{i}\left(p_{i} j_{i} m_{i} \lambda_{j}^{\prime} \lambda_{k}^{\prime}\right) \lambda_{i}^{\prime} ; q_{k}\left(p_{k} j_{k} m_{k} \lambda_{i} \lambda_{j}\right) \lambda_{k}\right\}
\end{aligned}
$$

An equivalent definition can be used for the off-mass-shell recoupling coefficients.

If the scattering operator is rotationally invariant in the isospin space, then $\left[S, U\left(R_{I}\right)\right]=0 \quad\left(R_{I} \in S U(2)_{\text {isospin }}\right)$. As with rotational invariance this implies that the scattering aplitude 
$\left\langle T^{\prime} M^{\prime}|S| T M\right.$, where $T$ is the total isospin and $M$ is its projection, is proportional to $\delta_{T^{\prime} T} \delta_{M^{\prime} M}$ and is independent of $M$. The isospin properties of the $M$ function are identical to those of the scattering amplitude. 
Appendix A

Miscellaneous Results Concerning Rotation Matrices and Clebsch-Gordan Coefficients ${ }^{41}$

$$
\begin{aligned}
& D_{m^{\prime} m}^{j}(R(\theta, \hat{y})) \equiv d_{m^{\prime} m}^{j}(\theta) \\
& (A-1) \\
& d_{m^{\prime} m}^{j}(\theta)=\left\{\frac{(j+m) !(j-m) !}{\left(j+m^{\prime}\right) !\left(j-m^{\prime}\right) !}\right\}\left(\sin \frac{\theta}{2}\right)^{m-m^{\prime}} \\
& \times\left(\cos \frac{\theta}{2}\right)^{m+m^{\prime}} P_{j-m}^{\left(m-m^{\prime}, m^{\prime}+m^{\prime}\right)}(\cos \theta) \\
& (A-2) \\
& P_{\ell}^{\left(m_{1}, m_{2}\right)}(x)=\left(\ell+m_{1}\right) !\left(\ell+m_{2}\right) ! \\
& \times \sum_{n} \frac{\left(\frac{x-1}{2}\right)^{l-n}\left(\frac{x+1}{2}\right)^{n}}{n !\left(l+m_{1}-n\right) !\left(m_{2}+n\right) !(l-n) !} \\
& (A-3) \\
& d_{m^{\prime} m}^{j}(\pi)=(-1)^{j-m} \delta_{m^{\prime},-m} \\
& (A-4) \\
& d_{m^{\prime} m}^{j}(-\theta)=d_{m m^{\prime}}^{j}(\theta)=(-1)^{m^{\prime}-m} d_{m^{\prime} m}^{j}(\theta) \\
& d_{-m^{\prime},-m}^{j}(\theta)=d_{m m^{\prime}}^{j}(\theta) \\
& (A-6)
\end{aligned}
$$

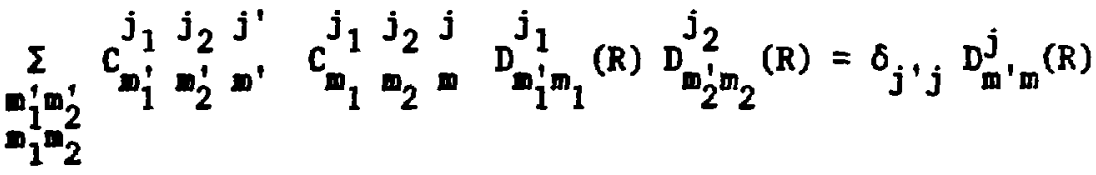

197 


$$
\begin{aligned}
& W\left(j_{2} j_{3} j_{1} J ; J_{1} J_{3}\right) \\
& =(-1)^{j_{3}+J-J_{3}}\left(\left(2 J_{1}+1\right)\left(2 J_{3}+1\right)\right)^{-\frac{1}{2}}
\end{aligned}
$$

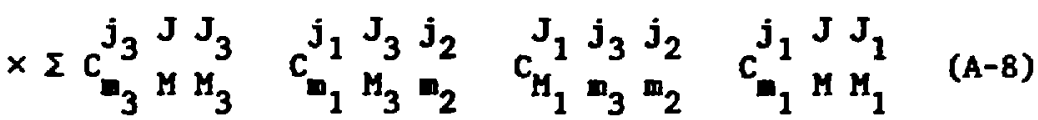

(sum over $m_{1}$ and $m_{3} ; m_{2}$ can be chosen arbitrarily) 
Appendix $B$

$\underline{\text { SO}(3) \leftrightarrow S U(2)}$

The proof of Eq. 1-10 proceeds as follows:

Let $x_{i}^{\prime}=\sum_{j} r_{i j} x_{j}$

in the equation for the similarity transformation of the $2 \times 2$ matrix $\vec{x} \cdot \overrightarrow{0}$ (Eq. 1-9)

$$
\vec{x} \cdot \vec{\sigma}=A \vec{x} \cdot \vec{\sigma} A^{\dagger}
$$

where $A$ is any unitary matrix. This equation and the properties of the Pauli matrices (Egs. 1-5 and 1-7) then inply that

$$
r_{i j}=\frac{1}{2} \operatorname{tr}\left(\sigma_{i} A \sigma_{j} A^{\dagger}\right)
$$

Hence, $r$ is real and orthogonal.

i.e., $r_{i j}^{*}=r_{i j}$

and $\operatorname{det} \vec{x}^{\prime} \cdot \vec{\sigma}=\operatorname{det} A \vec{x} \cdot \vec{\sigma} A^{\dagger}=\operatorname{det} \vec{x} \cdot \vec{\sigma}$

which implies that $\overrightarrow{\mathbf{x}} \cdot \overrightarrow{\vec{x}} \cdot=\vec{r} \vec{x} \cdot \mathbf{r} \vec{x}=\vec{x} \cdot \vec{x}$

so that $r^{T}=r r^{T}=1$. 
The $3 \times 3$ matrix $r$ is also proper (i.e., det $r=1$ ) as can be seen from the following proof:

Define the column vector

$$
n \equiv\left(\begin{array}{c}
-x_{3} \\
-x_{1}+i x_{2} \\
-x_{1}-i x_{2} \\
x_{3}
\end{array}\right)
$$

so that, $\eta^{\prime}=\left(A \times A^{*}\right) \eta$ where $A \times A^{*}$ is the $4 \times 4$ direct-product matrix of $A$ and $A^{*}$. Next let

$$
x \equiv\left(\begin{array}{l}
0 \\
x_{1} \\
x_{2} \\
x_{3}
\end{array}\right)=\frac{1}{\sqrt{2}} \text { s n }
$$

with $\quad S \equiv \frac{1}{\sqrt{2}}\left(\begin{array}{rrrr}1 & 0 & 0 & 1 \\ 0 & -1 & -1 & 0 \\ 0 & -i & i & 0 \\ -1 & 0 & 0 & 1\end{array}\right)$

so that $S s^{\dagger}=s^{\dagger} S=1$, which implies that

$$
x^{\prime}=S\left(A_{A}^{*}\right) S^{\dagger} x \equiv l x
$$

with $\ell \equiv S\left(A \times A^{*}\right) S^{\dagger}$ 
and $\operatorname{det} l=\operatorname{det}\left(A \times A^{*}\right)=(\operatorname{det} A)^{2}\left(\operatorname{det} A^{*}\right)^{2}=\left(\operatorname{det} M^{\dagger}\right)^{2}$

Thus, if $\operatorname{det} A= \pm 1$ or if $A$ is unitary, then $\operatorname{det} l=+1$. This result can then be inserted into Eq. B-2, which in terms of the Datrix $\ell$ is

$$
\begin{aligned}
\left(\ell_{00} x_{0}+\ell_{0 j} x_{j}\right) \sigma_{0}- & \left(l_{i 0} x_{0}+\ell_{i j} x_{j}\right) \sigma_{i} \\
& =x_{0} A O_{0} A^{\dagger}-x_{i} A \sigma_{i} A^{\dagger}
\end{aligned}
$$

(a sum over repeated indices is implied), to obtain

$$
\begin{aligned}
& \varepsilon_{00}=\frac{1}{2} \operatorname{tr} A A^{\dagger} \\
& \varepsilon_{0 i}=-\frac{1}{2} \operatorname{tr} A \sigma_{i} A^{\dagger} \\
& \varepsilon_{i 0}=-\frac{1}{2} \operatorname{tr} \sigma_{i} A A^{\dagger} \\
& \varepsilon_{i j}=\frac{1}{2} \operatorname{tr} \sigma_{i} A \sigma_{j} A^{\dagger}=x_{i j}
\end{aligned}
$$

or $\quad \ell_{\mu v}=\frac{1}{2} \operatorname{tr} \tilde{\sigma}_{\mu} A \tilde{\sigma}_{v} A$

Hence, if $A$ is unitary, then

$$
l=\left(\begin{array}{cccc}
1 & 0 & 0 & 0 \\
0 & r^{-} & -? \\
0 & 1 & r & \vdots \\
0 & 2 & - & -j
\end{array}\right)
$$

and $\operatorname{det} \ell=\operatorname{det} r=+1$. (End of proof.) 
Thus, a $2 \times 2$ unitary natrix in Eq. B-2 determines a unique $3 \times 3$ real, proper, and orthogonal natrix $r$ as defined in Eq. B-1. Furthermore, Eq. B-11 implies that if $A_{1}$ determines $\ell_{1}$ and $A_{2}$ deteraines $\ell_{2}$, then $A_{1} A_{2}$ determines $\ell_{1} \ell_{2}$. Hence, $r$ is an element of the group $50(3)$.

Eq. B-3 can also be inverted to determine the $2 \times 2$ matrix A that corresponds to the $3 \times 3$ matrix $r$. This inversion is accomplished by noting that

$$
\operatorname{tr} l=1+\operatorname{tr} r=\operatorname{tr} S\left(A \times A^{*}\right) S^{\dagger}=(\operatorname{tr} A)\left(\operatorname{tr} A^{*}\right)
$$

and $\quad \sum_{i j} r_{i j} \sigma_{i} \sigma_{j}=2 A \operatorname{tr} A^{*}-\sigma_{0}$

where Eq. B-19 is proven as follows:

Because Eq. B-2 is valid for any vector $\vec{x}$, the following equality nust be true:

$$
\sum_{i j} r_{i j} \sigma_{i} \sigma_{j}=\sum_{j} A \sigma_{j} A^{\dagger} \sigma_{j}
$$

And, because the Pauli matrices are a complete set of $2 \times 2$ matrices, the atrix $A^{\dagger}$ can be written as

$$
A^{\dagger}=a_{0} \sigma_{0}+\vec{a} \cdot \vec{\sigma}
$$


These equations and Eq. 1-7 then imply that

$$
\sum_{j} \sigma_{j} A^{\dagger} \sigma_{j}=4 a_{0} \sigma_{0}-A^{\dagger}
$$

But, $\operatorname{tr} A^{\dagger}=2 a_{0}$

Hence, $\quad \sum_{j} \sigma_{j} A^{\dagger} \sigma_{j}=2\left(\operatorname{tr} A^{\dagger}\right) \sigma_{0}-A^{\dagger}$

or $\sum_{j} A \sigma_{j} A^{\dagger} \sigma_{j}=2 A\left(\operatorname{tr} A^{\frac{1}{*}}\right)-\sigma_{0}$

Thus, $\sum_{i j} r_{i j} \sigma_{i} \sigma_{j}=2 A\left(\operatorname{tr} A^{*}\right)-\sigma_{0}$

(End of proof.)

Equations $B-18, B-19, B-21$, and $B-23$ can now be used to show that

$$
1+\operatorname{tr} r=4\left|a_{0}\right|^{2}
$$

and $\sum_{i j} r_{i j} \sigma_{i} \sigma_{j}=4 a_{0} A-\sigma_{0}$

Hence, if $\operatorname{tr} I \neq 1$ and $\star_{0}$ is real, then

$$
A=\frac{1}{2(1+t r)^{\frac{1}{2}}}\left(\sigma_{0}+\sum_{i j} r_{i j} \sigma_{i} \sigma_{j}\right)
$$


For example, $r_{i j}$ can be written as (Eq. 1-3)

$$
r(\phi, \hat{n})_{i j}=\delta_{i j} \cos \phi+n_{i j} n_{j}(1-\cos \phi)+\varepsilon_{i k j} n_{k} \sin \phi
$$

which implies that

$$
A=\sigma_{0} \cos \frac{\phi}{2}-i \hat{n} \cdot \vec{\sigma} \sin \frac{\phi}{2}
$$

Furthermore, if $\operatorname{tr} r=-1$, then $\phi$ is equal to $\pi$, which implies that

$$
A=-i \hat{n} \cdot \vec{\sigma}
$$

Thus, the solutions of Eq. B-3 are given by the $2 \times 2$ matrix in Eqs. B-31 and B-32 and any phase multiple of that matrix. However, if $\operatorname{det} A=+1$, then only $A$ and $-A$ are allowed solutions. (Note: $\operatorname{det} e^{i \phi} A=e^{2 i \phi} \operatorname{det} A$ ). 
Appendix $\mathrm{C}$

Details from Chapter 3

C-1 Proof of Eg. 3-5

Eqs. 3-3, 3-4, and the Lorentz invariance of the scalar product imply that

$$
K \cdot k_{1}=E \varepsilon_{1}-\vec{k} \cdot \vec{K}_{1}=u w_{1}
$$

which implies that

$$
\vec{k} \cdot \vec{p}=w_{2} \varepsilon_{1}-w_{1} \varepsilon_{2}
$$

This result and Eq. 3-4 then imply that

$$
\left(\varepsilon_{2}+w_{2}\right) \overrightarrow{\mathrm{K}}_{1}-\left(\varepsilon_{1}+w_{1}\right) \overrightarrow{\mathrm{K}}_{2}=(E+w) \vec{p}
$$

Thus, $\quad \vec{p}=\frac{1}{E+w}\left[\left(\varepsilon_{2}+w_{2}\right) \vec{K}_{1}-\left(\varepsilon_{1}+w_{1}\right) \vec{K}_{2}\right]$

C-2 Proof of Eg. 3-7

Consider $\quad w=\left(p^{2}+m_{1}^{2}\right)^{\frac{1}{2}}+\left(p^{2}+m_{2}^{2}\right)^{\frac{1}{2}}$

and let $x \equiv \frac{1}{2}\left(\omega^{2}-m_{1}^{2}-m_{2}^{2}\right)$;

$(C-6)$

205 
so that, $\quad x^{2}=m_{1}^{2} p^{2}+m_{2}^{2} p^{2}+m_{1}^{2} m_{2}^{2}+2 p^{2} x$

This implies that

$$
p^{2}=\frac{1}{w^{2}}\left(\frac{1}{4}\left(w^{2}-m_{1}^{2}-m_{2}^{2}\right)^{2}-m_{1}^{2} m_{2}^{2}\right)
$$

or $\quad p=\frac{1}{2 w} \xi\left(w^{2}, m_{1}^{2}, m_{2}{ }^{2}\right)$

where $\xi(x, y, z)=\left[(x-y-z)^{2}-4 y z\right]^{\frac{1}{2}}$

\section{C-3 Proof of Egs. 3-34 and 3-37}

Consider $(\$ 2.3)$

$$
\left|\stackrel{\circ}{\mathrm{K}}\left(\overrightarrow{\mathrm{p}} \lambda_{1} \lambda_{2}\right)>\equiv U\left(L\left(x_{k_{1}}, \hat{\mathrm{p}}\right)\right)\right| \stackrel{\circ}{\mathrm{k}_{1}} \lambda_{1}>\otimes U\left(L\left(x_{k_{2}},-\hat{\mathrm{p}}\right)\right) \mid \stackrel{\circ}{\mathrm{k}_{2}} \lambda_{2}>
$$

and $\quad U\left(J_{s}\right) U(L)=U\left(\zeta L^{*} \zeta^{\dagger}\right) U\left(J_{s}\right)$

with $\quad \zeta L(x, \hat{\mathrm{n}})^{*} \zeta^{\dagger}=I(x,-\hat{\mathrm{n}})$.

These equations imply that

$$
\begin{aligned}
& U\left(d_{s}\right)\left|\stackrel{\circ}{K}\left(p \quad \lambda_{1} \lambda_{2}\right)>=\eta_{1} \eta_{2} U\left(L\left(x_{k_{1}},-\hat{p}\right)\right)\right| \dot{k}_{1} \lambda_{1}>\otimes U\left(L\left(x_{k_{2}}, \hat{p}\right)\right) \mid k_{2} \lambda_{2}> \\
& =\left|\stackrel{i}{K}\left(\begin{array}{llll}
-\vec{p} & \lambda_{1} & \lambda_{2}
\end{array}\right)\right\rangle
\end{aligned}
$$

and (see Eq. 3-21) 


$$
\begin{aligned}
& U\left(f_{s}\right) \mid \dot{K}\left(p \&=\lambda_{1} \lambda_{2}\right) z_{z}=\eta_{1} \eta_{2}\left(\frac{2 \ell+1}{4 \pi}\right)^{\frac{1}{2}} \int_{0}^{2 \pi} d \phi \int_{0}^{\pi} d \theta \sin \theta \\
& \times D_{m 0}^{\ell}(R(\phi, \theta))^{*} \sum_{\ell^{\prime} m^{\prime}}\left(\frac{2 \ell^{\prime}+1}{4 \pi}\right)^{\frac{1}{2}} D_{m^{\prime} 0^{\prime}}^{\ell^{\prime}}(R(\phi+\pi, \pi-\theta)) \mid \stackrel{0}{K}\left(p \ell^{\prime} m^{\prime} \lambda_{1} \lambda_{2}\right)_{2}{ }^{\prime}
\end{aligned}
$$

(Note: If $\phi>\pi$, the angle in the second $D$ matrix in this equation should be $\phi-\pi$; however, $D_{m 0}^{l}(R(\phi-\pi, \hat{z}))=e^{2 \pi i m} D_{m 0}^{l}(R(\phi+\pi, \hat{z}))$ and, because $\ell$ is an interger, $e^{2 \pi i m}=+1$.)

But, $\quad R(\phi+\pi, \pi-\theta)=R(\phi, \theta) R(\pi, \pi)$

and $\mathrm{D}_{\mathrm{m} 0}^{\ell}(R(\pi, \pi))=\mathrm{e}^{-i \pi m}(-1)^{\ell-0} \delta_{\mathrm{mo}}=(-1)^{\ell}$

Hence, the integral in Eq. C-15 can be completed to obtain

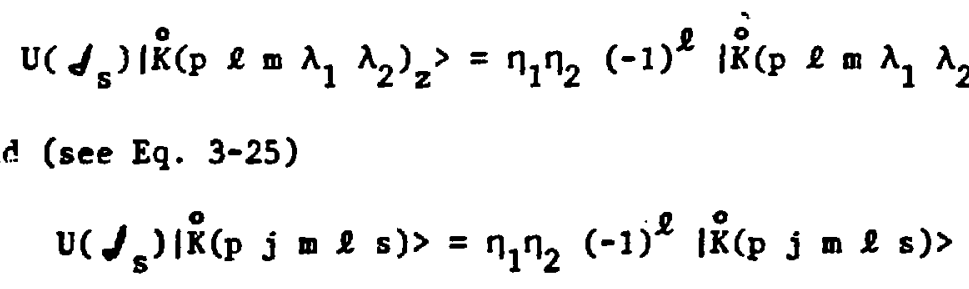

Eq. C-15 also implies that

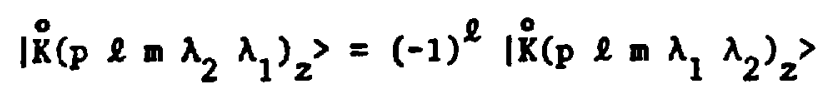

Hence (see Eq. 3-25),

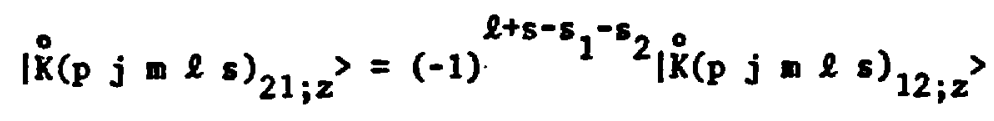


C-4 Proof of Eg. 3-36

Eqs. 3-29 and 3-35 imply that

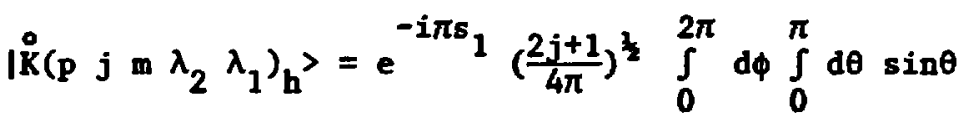

$$
\begin{aligned}
& \times D_{m, \lambda_{2}-\lambda_{1}}^{j}(R(\phi, \theta))^{*} v(R(\pi, \hat{z}))\left\{v \left(R(\phi+3 \pi, \theta) Z\left(x_{k_{2}}\right)\left|k_{2} \lambda_{2}\right\rangle\right.\right. \\
& \otimes u\left(R(\phi, \pi-\theta) z\left(x_{k_{1}}\right)\left\{i_{1} \lambda_{1}>\right\}\right. \\
& =e^{-i \pi s_{1}}(-1)^{2 \lambda_{2}}\left(\frac{2 j+1}{4 \pi}\right)^{\frac{1}{2}} \int_{0}^{2 \pi} d \phi \int_{0}^{\pi} d \theta \cos \theta D_{m, \lambda_{2}-\lambda_{1}}^{j}(R(\phi, \pi-\theta))^{\frac{*}{\pi}} \\
& \times v(R(\pi, \hat{z}))\left\{U\left(R(\phi, \theta) Z\left(x_{k_{1}}\right)\left|\dot{k}_{1} \lambda_{1}>\otimes U\left(R(\phi+\pi, \pi-\theta) Z\left(x_{k_{2}}\right)\right)\right| \dot{k}_{2} \lambda_{2}>\right\}\right.
\end{aligned}
$$

But,

$$
D_{m, \lambda_{2}-\lambda_{1}}^{j}(R(\phi, \pi-\theta))=e^{-i m \phi} d_{m, \lambda_{2}-\lambda_{1}}^{j}(\pi-\theta)=(-1)^{j+m} D_{m, \lambda_{1}-\lambda_{2}}^{j}(R(\phi, \theta))
$$

Hence, $\left.\quad \stackrel{\circ}{\mathrm{K}}\left(\mathrm{p} j \mathrm{j} \lambda_{2} \lambda_{1}\right)_{h}\right\rangle=e^{-i \pi s_{1}+i \pi s_{2}}(-1)^{2 \lambda_{2}+j+m}$

$$
\begin{aligned}
& \left.\times\left. U(R(\pi, \hat{z}))\right|_{K} ^{\circ}\left(p j=\lambda_{1} \lambda_{2}\right)_{h}\right\rangle \\
& =(-1)^{j-s_{1}-s_{2}} \mid K\left(p j \neq \lambda_{1} \lambda_{2}\right)_{h}>
\end{aligned}
$$




\section{C-5 Proof of $\mathrm{Eg} . \quad 3-38$}

Consider (\$2.3)

$$
\left|\stackrel{0}{\mid \mathrm{K}}\left(\overrightarrow{\mathrm{p}} \lambda_{1} \lambda_{2}\right)_{\mathrm{h}}\right\rangle \equiv \mathrm{U}\left(\mathrm{R}(\hat{\mathrm{p}}) \mathrm{Z}\left(\mathrm{x}_{\mathrm{k}_{1}}\right)\left|\dot{\mathrm{k}}_{1} \lambda_{1}>\Theta \mathrm{U}\left(\mathrm{R}(\hat{\mathrm{p}}) \mathrm{R}(\pi, \pi) \mathrm{Z}\left(\mathrm{x}_{\mathrm{k}_{2}}\right)\right)\right| \dot{\mathrm{k}}_{2} \lambda_{2}\right\rangle
$$

and $\mathrm{U}\left(\mathcal{d}_{\mathrm{s}}\right) \mathrm{U}(\mathrm{L})=\mathrm{U}\left(\zeta \mathrm{L}^{2} \zeta^{\dagger}\right) \mathrm{U}\left(\mathcal{L}_{\mathrm{s}}\right)$

with $\quad \zeta R(\hat{\mathrm{p}})^{\star} \mathrm{Z}(\mathrm{x})^{*} \zeta^{\dagger}=\mathrm{R}(\hat{\mathrm{p}}) \mathrm{R}(\pi, \hat{\mathrm{y}}) \mathrm{Z}(\mathrm{x}) \mathrm{R}(\pi, \hat{\mathrm{y}})^{-1}$

and $\zeta R(\hat{\mathrm{p}})^{*} \mathrm{R}(\pi, \pi)^{*} \mathrm{Z}(\mathrm{X})^{*} \zeta^{\dagger}=\mathrm{R}(\hat{\mathrm{p}}) \mathrm{R}(\pi, 2 \pi) Z(\chi) R(\pi, \hat{\mathrm{y}})^{-1}$

These equations imply that

$$
\begin{aligned}
& v\left(d_{s}\right)\left|\stackrel{\circ}{K}\left(\vec{p} \lambda_{1} \lambda_{2}\right)_{h}\right\rangle=\eta_{1} \eta_{2}(-1)^{s_{1}+\lambda_{1}}(-1)^{s_{2}+\lambda_{2}} \\
& \times \quad U\left(R(\hat{p}) R(\pi, \hat{y}) Z\left(x_{k_{1}}\right) \mid \dot{k}_{1}-\lambda_{1}>\otimes U\left(R(\hat{p}) R(\pi, 2 \pi) Z\left(x_{k_{2}}\right) \mid i_{2}-\lambda_{2}>\right.\right. \\
& =\eta_{1} \eta_{2}(-1)^{s_{1}+\lambda_{1}}(-1)^{s_{2}+\lambda_{2}} \mathrm{U}(R(\pi, \hat{y})) \\
& \times\left\{u \left(R(-\phi, \theta) Z\left(x_{k_{1}}\right) \mid \dot{k}_{1}-\lambda_{1}>\otimes u\left(R(-\phi, \theta) R(-\pi, \pi) z\left(x_{k_{2}}\right) \mid \stackrel{0}{k_{2}}-\lambda_{2}>\right\}\right.\right.
\end{aligned}
$$




\section{Hence,}

$$
\begin{aligned}
& u\left(d_{s}\right) i K\left(p j=\lambda_{1} \lambda_{2}\right)_{h}>=n_{1} \eta_{2}(-1)^{s_{1}+\lambda_{1}}(-1)^{s_{2}+\lambda_{2}} e^{-2 \pi i \lambda_{2}}\left(\frac{2 j+1}{4 \pi}\right)^{\frac{3}{2}} \\
& \times \int_{0}^{2 \pi} d \phi \int_{0}^{\pi} d \theta \sin \theta D_{m, \lambda_{1}-\lambda_{2}}^{j}(R(\phi, \theta))^{*} \mathrm{v}(R(\pi, \hat{y}) R(-2 \phi, \hat{z})) \\
& \times \sum_{j^{\prime} m^{\prime}}\left(\frac{2 j^{\prime}+1}{4 \pi}\right)^{\frac{1}{2}} D_{m^{\prime},-\lambda_{1}+\lambda_{2}}^{j^{\prime}}(R(\phi, \theta)) \mid \stackrel{0}{k}\left(p j^{\prime} m^{\prime}-\lambda_{1}-\lambda_{2}\right)_{h^{\prime}}
\end{aligned}
$$

But, $\quad R(\pi, \hat{y}) R(-2 \phi, \hat{z})=R(2 \phi, \pi)$

and $U(R(2 \phi, \pi)) \mid \stackrel{0}{K}\left(p j^{\prime} m^{\prime}-\lambda_{1}-\lambda_{2}\right)_{h^{\prime}}=(-1)^{j^{\prime}-m^{\prime}} e^{2 i \phi m^{\prime}}$

$$
\times \quad \stackrel{0}{\mid K}\left(p j^{\prime}-m^{\prime}-\lambda_{1} \lambda_{2}\right)_{h}>
$$

and $e^{2 i \phi m^{\prime}} D_{m^{\prime},-\lambda_{1}+\lambda_{2}}^{j^{\prime}}(R(\phi, \theta))=(-1)^{m^{\prime}+\lambda_{1}-\lambda_{2}} D_{-m^{\prime}, \lambda_{1}-\lambda_{2}}^{j^{\prime}}(R(\phi, \theta))$

Thus,

$$
U\left(\mathcal{J}_{s}\right)\left|\stackrel{0}{K}\left(\mathrm{p} j \mathrm{j} \lambda_{1} \lambda_{2}\right)_{h}\right\rangle=\eta_{1} \eta_{2}(-1)^{j-s_{1}-s_{2}}\left|\stackrel{0}{K}\left(\mathrm{p} j \mathrm{~m}-\lambda_{1}-\lambda_{2}\right)_{h}\right\rangle
$$


C-6 Proof of Eg. 3-41

The results of $\$ 2.3 .2$ imply that

$$
U\left(J_{t}\right)\left|\stackrel{0}{K}\left(\vec{p} \lambda_{1} \lambda_{2}\right)_{2}\right\rangle=(-1)^{s_{1}-\lambda_{1}}(-1)^{s_{2}-\lambda_{2}}\left|\stackrel{0}{K}\left(-\vec{p}-\lambda_{1}-\lambda_{2}\right)_{2}\right\rangle \quad(c-35)
$$

which implies that

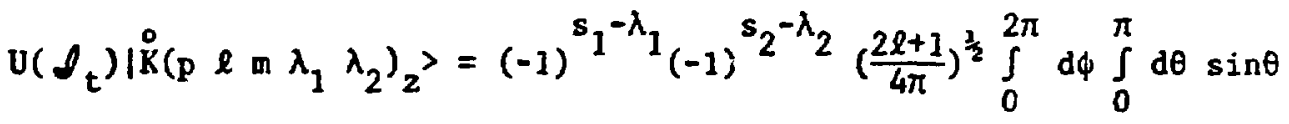

$$
\begin{aligned}
& \times D_{m 0}^{\ell}(R(\phi, \theta)) \sum_{\ell^{\prime} m^{\prime}} D_{m^{\prime} 0}^{\ell^{\prime}}(R(\phi+\pi, \pi-\theta)) \mid \stackrel{0}{K}\left(p \ell^{\prime} m^{\prime}-\lambda_{1}-\lambda_{2}\right) z^{\prime}
\end{aligned}
$$

But, $\quad D_{m^{\prime} 0}^{\ell^{\prime}}(R(\phi+\pi, \pi-\theta))=(-1)^{\ell^{\prime}-m^{\prime}} D_{-m^{\prime} 0}^{\ell^{\prime}}(R(\phi, \theta))^{*}$

Hence, the integral in Eq. C-36 can be completed to obtain

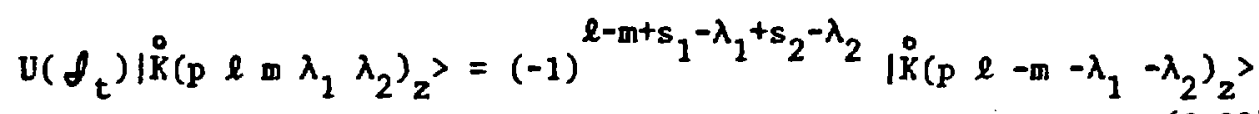

This result can then be inserted into Eq. 3-25 to obtain

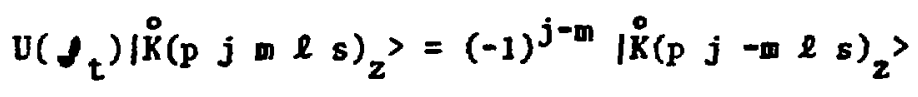


Appendix D

Field Theory 28,45

D-1 The Fields

The state vector $|p \lambda\rangle$ was defined in Chapter 2 with the following normalization:

$$
\left\langle p^{\prime} \lambda^{\prime} \mid p \lambda\right\rangle=2 \varepsilon \delta^{3}\left(\vec{p}^{\prime}-\vec{p}\right) \delta \lambda_{\lambda} \lambda
$$

where $\varepsilon \equiv\left(|\vec{p}|^{2}+m^{2}\right)^{\frac{1}{2}}$. Under a Poincare transformation,

$$
\left.U(P(a, L))|p \lambda\rangle=e^{i p \cdot a} \sum_{v} D_{v \lambda}^{s}(R(L ; p)) \mid \ell p v\right)
$$

where $R(L ; p) \equiv C(\ell p)^{-1} L C(p)$ and $C(p)$ is a complementary set Lorentz transformation.

The creation operator $a(p \lambda)^{\dagger}$ will be defined by the following relation:

$$
(p \lambda)^{\dagger}|0\rangle=|p \lambda\rangle
$$


where $|0\rangle$ is the vacuum state vector. The annihilation operator a $(p \lambda)$ returns the single-particle state to the vacuum and is defined by

$$
\left[a\left(p^{\prime} \lambda^{\prime}\right), a(p \lambda)^{\dagger}\right]_{ \pm}=2 \varepsilon \delta^{3}\left(\vec{p}^{\prime}-\vec{p}\right) \delta_{\lambda \cdot \lambda}
$$

where the normalization of Eq. D-I has been used, and the \pm indicates that this is an anticommutator ('+') or commutator ('-'). The determination of whether a commutator or anticommutator should be used will be discussed in \$D-2. The Poincaré transformation property of the operator $a(p \lambda)^{\dagger}$ is determined from Eq. D-2.

$$
U(P(a, L)) a(p \lambda)^{\dagger} U(P(a, 1))^{\dagger}=e^{i p \cdot a} \sum_{v} D_{v \lambda}^{s}(R(L ; P)) a(\ell p v)^{\dagger}
$$

The field operator $\phi_{\lambda}(x)$ will be constructed as a linear combination of the annibilation operators.

i.e., $\quad \phi_{\lambda}(x) \equiv \sum_{v} \int \frac{d^{3} p}{(2 \pi)^{3 / 2} 2 \varepsilon} F_{\lambda \nu}(\vec{p}, x) a(p v)$

This field will be defined to have the following Poincaré transformation property:

$$
U(\rho(a, L)) \phi_{\lambda}(x) U(P(a, L))^{\dagger}=\sum_{\lambda^{\prime}} D_{\lambda^{\prime}}^{s}\left(L^{-1}\right) \phi_{\lambda^{\prime}}(l x+a)
$$


The function $F_{\lambda v}(\vec{p}, x)$ in $E q . D-6$ is determined from this definition and the Poincaré transformation property of the annihilation operator as follows:

i) The translation properties of the operators $a(p \lambda)$ and $\phi_{\lambda}(x)$ imply that

$$
F_{\lambda v}(\vec{p}, x+a)=e^{-i p \cdot a} F_{\lambda v}(\vec{p}, x)
$$

so that,

$$
F_{\lambda v}(\vec{p}, x)=e^{-i p \cdot x} F_{\lambda v}(\vec{p})
$$

where $\quad F(\vec{p}) \equiv F(\vec{p}, 0)$

ii) The Lorentz transformation properties of the operators a $(p \lambda)$ and $\phi_{\lambda}(x)$ and the Lorentz invariance of the volume element $\left(d^{3} p / 2 \varepsilon\right)$ imply that

$$
\sum_{v} F_{\lambda \nu}(\vec{p}) D_{v \mu}^{s}\left(R(L ; p)^{-1}\right)=\sum_{v} D_{\lambda \nu}^{s}\left(L^{-1}\right) F_{v \mu}(\overrightarrow{l p})
$$

But, $R(L ; p)=C(\ell p)^{-1} L C(p)$, which implies that (in natrix notation)

$$
F(\vec{p}) D\left(C(p)^{-1}\right) D\left(L^{-1}\right)=D\left(L^{-1}\right) F(\overrightarrow{\ell p}) D\left(C(\ell p)^{-1}\right)
$$


Now let $p=\dot{p}(i . e ., \vec{p}=0)$ and let $L$ be an arbitrary rotation $R$; so that,

$$
F(\vec{D}) D\left(R^{-1}\right)=D\left(R^{-1}\right) I(\vec{D})
$$

This implies that (Schurr's lemma).

$$
F_{\lambda \nu}(\overrightarrow{0})=c \delta_{\lambda \nu}
$$

where $c$ is some constant. Then let $p=\stackrel{0}{p}$ and $p^{\prime}=\operatorname{lp}$ with $I \equiv$ $C\left(p^{\prime}\right)$; so that,

$$
F(\overrightarrow{0}) D\left(C\left(p^{\prime}\right)^{-1}\right)=D\left(C\left(p^{\prime}\right)^{-1}\right) F\left(\vec{p}^{\prime}\right) D\left(C\left(p^{\prime}\right)^{-1}\right)
$$

Hence, $\quad F_{\lambda \nu}\left(\vec{p}^{\prime}\right)=c D_{\lambda \nu}^{s}\left(C\left(p^{\prime}\right)\right)$

and if $c$ is set equal to 1 (i.e., if the normalization is chosen such that $c=1$ ), then

$$
\phi_{\lambda}(x)=\sum_{v} \int \frac{d^{3} p}{(2 \pi)^{3 / 2} 2 \varepsilon} D_{\lambda v}^{s}(c(p)) e^{-i p \cdot x} \cdot(p v)
$$

In addition to the $2 s+1$ dimensional representation of $\operatorname{SL}(2, C) D^{S}(I)$, there exists one other inequivalent irredicible representation of the same dimension. This representation can be 
chosen to be

$$
\bar{D}(L) \equiv D\left(\zeta L^{*} \zeta^{\dagger}\right)=D\left(\left(L^{-1}\right)^{\dagger}\right)
$$

where $\zeta \equiv R(\pi, \hat{y})$ (see $\$ 2.3$ ). The field that is constructed from this representation will be denoted by $x_{\lambda}(x)$ and has the following Poincaré transformation property:

$$
\begin{aligned}
U(\rho(a, L)) x_{\lambda}(x) U(P(a, I))^{\dagger} & =\sum_{v} \bar{D}_{\lambda v}^{s}\left(L^{-1}\right) x_{v}(l x+a) \\
& =\sum_{v} D_{\lambda v}^{s}\left(L^{\dagger}\right) x_{v}(l x+a)
\end{aligned}
$$

The procedure shown in Eqs. D-8 through D-15 can then be followed to show that this field can be written as

$$
x_{\lambda}(x)=\sum_{v} \int \frac{d^{3} p}{(2 \pi)^{3 / 2} 2 \varepsilon} \bar{D}_{\lambda v}^{s}(c(p)) e^{-i p \cdot x} a(p v)
$$

(Note: The matrix $D^{S}(L)$ is called the " $(S, 0)$ representation" of $\operatorname{SL}(2, C)$ and $D^{S}(L)$ is called the " $(0, S)$ representation" of $\left.\operatorname{SL}(2, C).\right)$ 


\section{D-2 Causality}

It is now assuned that any two fields $A(x)$ and $B(y)$ must commute or anticommute if the separation $(x-y)$ is spacelike (i.e., $\left.(x-y)^{2}<0\right)$. In this section, it will be shown that a consequence of this assumption is the Pauli exclusion principle (i.e., halfinteger-spin fields anticommute and integer-spin fields commute). The connutators/anticomntators for the fields are (see Eq. 4-13)

$$
\begin{aligned}
& {\left[\phi_{\lambda}(x), \phi_{\lambda},(y)^{\dagger}\right]_{ \pm}=\frac{1}{(2 \pi)^{3}} \int \frac{d^{3} p}{2 \varepsilon} D_{\lambda \lambda^{\prime}}^{s}\left(\frac{\sigma \cdot p}{m}\right) e^{-i p \cdot(x-y)}} \\
& {\left[\phi_{\lambda}(x), x_{\lambda^{\prime}}(y)^{\dagger}\right]_{ \pm}=\frac{1}{(2 \pi)^{3}} \int \frac{d^{3} p}{2 \varepsilon} \delta_{\lambda \lambda^{\prime}} e^{-i p \cdot(x-y)}} \\
& {\left[x_{\lambda}(x), \phi_{\lambda}(y)^{\dagger}\right]_{ \pm}=\frac{1}{(2 \pi)^{3}} \int \frac{d^{3} p}{2 \varepsilon} \delta_{\lambda \lambda^{\prime}} e^{-i p \cdot(x-y)}} \\
& {\left[x_{\lambda}(x), x_{\lambda} \cdot(y)^{\dagger}\right]_{ \pm}=\frac{1}{(2 \pi)^{3}} \int \frac{d^{3} p}{2 \varepsilon} \bar{D}_{\lambda \lambda^{\prime}}^{s}\left(\frac{\sigma \cdot p}{m}\right) e^{-i p \cdot(x-y)}}
\end{aligned}
$$

All other comutators/ anticomutators are zero, and it can be seen that these fields do not satisfy the causality requirement.

To obtain a set of fields that do satisfy this requirenent, consider the following linear combinations of the field operators: 


$$
A_{\lambda}(x) \equiv \alpha \phi_{\lambda}(x)+\beta \sum_{v} D_{\lambda v}^{s}\left(\zeta^{\dagger}\right) x_{v}(x)^{\dagger}
$$

and $\quad B_{\lambda}(x) \equiv \gamma x_{\lambda}(x)+\delta \sum_{v} D_{\lambda v}^{s}(\xi) \phi_{v}(x)^{\dagger}$

where $\alpha, \beta, \gamma$, and $\delta$ are constants. These fields have been defined such that $A_{\lambda}(x)$ Poincaré transforms as in Eq. D-7 and $B_{\lambda}(x)$ transforms as in Eq. D-19. The commutators/anticomutators for these fields can be evaluated using Eqs. D-21 through D-24. For example,

$$
\begin{aligned}
{\left[A_{\lambda}(x), A_{\lambda},(y)\right]_{ \pm}=} & \frac{\alpha \beta}{(2 \pi)^{3}} \int \frac{d^{3} p}{2 \varepsilon} D_{\lambda \lambda^{\prime}}^{s}(\zeta) \\
& \times\left\{e^{-i p \cdot(x-y)} \pm(-1)^{2 s} e^{+i p \cdot(x-y)}\right\}
\end{aligned}
$$

(Note: $D^{s}\left(\zeta^{\dagger}\right)=D^{S}(-\zeta)=(-1)^{2 s} D^{S}(\zeta)$.) Hence, if the commutator is used when $s$ is an integer and the anticosmutator is used when $s$ is half-integer, then

$$
\left[A_{\lambda}(x), A_{\lambda},(y)\right]_{ \pm}=i \alpha \beta D_{\lambda \lambda^{\prime}}^{\delta},(\xi) \Delta(x-y)
$$

where $\quad \Delta(x) \equiv \frac{-i}{(2 \pi)^{3}} \int \frac{d^{3} p}{2 \varepsilon}\left(e^{-i p \cdot x}-e^{+i p \cdot x}\right)$ 
$\Delta(x)$ is called the "Schwinger $\Delta$ function" 24 and is zero if $x^{2}<0$. As another example, consider the commutator/anticomutator of $A_{\lambda}(x)$ and $B_{\lambda},(y)$.

$$
\begin{aligned}
{\left[A_{\lambda}(x), B_{\lambda},(y)\right]_{ \pm}=} & \frac{1}{(2 \pi)^{3}} \int \frac{d^{3} p}{2 \varepsilon} D_{\lambda \lambda^{\prime}}^{\varepsilon}\left(\frac{\sigma \cdot p}{m} \xi^{\dagger}\right) \\
& \times\left\{\alpha \delta e^{-i p \cdot(x-y)} \pm \beta \gamma e^{+i p \cdot(x-y)}\right\}
\end{aligned}
$$

But, $D_{\lambda \lambda}^{s},\left(\frac{\sigma \cdot p}{m}\right)$ is polynomial in the components of the fourmomentum $p$, which implies that $p$ can be replaced with derivatives that act on the components of $(x-y)$.

i.e., $\quad p_{\mu} \rightarrow i \frac{\partial}{\partial(x-y)_{\mu}} \equiv i(\partial(x-y))_{\mu}$

Then, if $\alpha \delta=\beta y$ and the "correct" relation between the spin and the commutator/anticommutator is chosen,

$$
\left[A_{\lambda}(x), B_{\lambda},(y)\right]_{ \pm}=i \alpha \delta D_{\lambda \lambda^{\prime}}^{s}\left(\frac{i}{m} \sigma \cdot \partial(x-y) \zeta^{\dagger}\right) \Delta(x-y)
$$

Similar equations can be derived for the other commutators, and it is determined that $\alpha=\beta=\gamma=\delta=1$ when an appropriate 
normalization is chosen. Thus,

$$
\begin{array}{r}
A_{\lambda}(x)=\sum_{v} \int \frac{d^{3} p}{(2 \pi)^{3 / 2} 2 \varepsilon}\left\{D_{\lambda \nu}^{S}(C(p)) e^{-i p \cdot x} a(p v)\right. \\
\left.+D_{\lambda \nu}^{s}\left(C(p) \zeta^{\dagger}\right) e^{+i p \cdot x} a(p \quad v)^{\dagger}\right\}
\end{array}
$$

and

$$
\begin{array}{r}
B_{\lambda}(x)=\sum_{v} \int \frac{d^{3} p}{(2 \pi)^{3 / 2}}-\left\{\bar{D}_{\lambda \nu}^{s}(C(p)) e^{-i p \cdot x} a(p v)\right. \\
\left.+\bar{D}_{\lambda \nu}^{s}(C(p) \zeta) e^{+i p \cdot x} a(p v)^{\dagger}\right\}
\end{array}
$$

are inequivalent "causal" fields.

\section{D-3 Antiparticles}

If antiparticles are to be innluded in the theory, creation and annihilation operators can be defined for each (i.e., define $a(p \lambda)$ and $a(p \lambda)^{\dagger}$ for the particle and $b(p \lambda)$ and $b(p \lambda)^{\dagger}$ for the antiparticle), the fields $\phi_{\lambda}(x)$ and $x_{\lambda}(x)$ can be define? for the particle, and the fields $\bar{\phi}_{\lambda}(x)$ and $\bar{x}_{\lambda}(x)$ can be defined for the antiparticle by replacing $a(p \lambda)$ with $b(p \lambda)$ in Eqs. D-17 and D-20. 
Particle number conserving causal fields can then be defined as

$$
A_{\lambda}(x)=\phi_{\lambda}(x)+\sum_{\lambda}, D_{\lambda \lambda^{\prime}}^{s}\left(\xi^{\dagger}\right) \bar{x}_{\lambda^{\prime}}(x)^{\dagger}
$$

and $\quad B_{\lambda}(x)=x_{\lambda}(x)+\sum_{\lambda}, D_{\lambda \lambda^{\prime}}^{s}(\zeta) \bar{\phi}_{\lambda},(x)^{\dagger}$

This is the same as replacing $a(p \quad v)^{\dagger}$ with $b(p v)^{\dagger}$ in Eqs. D-33 and D-34.

D-4 Space Inversion, Time Reversal, and Charge Conjugation

Under the space-inversion transformation (cf., Eq. 2-58),

$$
U\left(J_{s}\right) a(p \lambda)^{\dagger} U\left(d_{s}\right)^{\dagger}=n_{p} \sum_{v} D_{v \lambda}^{s}\left(R_{s}(C(p))\right) a\left(I_{s} p v\right)^{\dagger} \quad(D-37)
$$

and $\quad U\left(\mathscr{Q}_{s}\right) b(p \lambda)^{\dagger} U\left(d_{s}\right)^{\dagger}=\bar{\eta}_{p} \sum_{v} D_{v \lambda}^{s}\left(R_{s}(C(p))\right) b\left(I_{s} p v\right)^{\dagger} \quad(D-38)$

where $\eta_{p}\left(\bar{\eta}_{p}\right)$ is the parity of the particle (antiparticle). This implies that

$$
U\left(\mathcal{J}_{s}\right) A_{\lambda}(x) U\left(\mathcal{J}_{s}\right)^{\dagger}=\eta_{p} B_{\lambda}\left(I_{s} x\right)
$$

provided $\bar{\eta}_{p}=(-1)^{2 s} \eta_{p}$ 
Under the time-reversal transformation (cf., Eq. 2-69),

$$
U\left(\rho_{t}\right) a(p \lambda)^{\dagger} U\left(\partial_{t}\right)^{\dagger}=\sum_{v} D_{v \lambda}^{s}\left(R_{s}(C(p)) \zeta\right) a\left(I_{s} p v\right)^{\dagger}
$$

$b(p \lambda)^{\dagger}$ also satisfies this equation. This implies that (remember that $U\left(d_{t}\right)$ is an antiunitary operator)

$$
v\left(d_{t}\right) A_{\lambda}(x) \cup\left(d_{t}\right)^{\dagger}=\sum_{v} D_{\lambda v}^{S}\left(\xi^{\dagger}\right) A_{v}\left(I_{t} x\right)
$$

This equation is also satisfied by $B_{\lambda}(x)$.

Under the charge-conjugation transformation (cf., \$4.4.1),

$$
u_{c} a(p \lambda)^{\dagger} u_{c}^{\dagger}=\eta_{c} b(p \lambda)^{\dagger}
$$

This implies that

$$
u_{c} A_{\lambda}(x) v_{c}^{\dagger}=\eta_{c} \sum_{v} D_{\lambda v}^{s}\left(\zeta^{\dagger}\right) B_{v}(x)^{\dagger}
$$




\section{D-5 2(2s+1) Component Fields}

Egs. D-39 and D-43 suggest that a $2(2 s+1)$ component field be defined as the direct sum of the fields $A_{\lambda}(x)$ and $B_{\lambda}(x)$.

i.e., $\quad \psi(x) \equiv\left(\begin{array}{l}A(x) \\ B(x)\end{array}\right)=A(x) \oplus B(x)$

This field can also be written as

$$
\begin{array}{r}
\psi_{\alpha}(x) \equiv \sum_{\lambda \beta} \int \frac{d^{3} p}{(2 \pi)^{3 / 2} 2 \varepsilon}\left\{O_{\alpha \beta}^{s}(c(p)) u_{\beta}(\lambda) e^{-i p \cdot x} a(p \lambda)\right. \\
\left.+O_{\alpha \beta}^{s}\left(c(p) \xi^{\dagger}\right) v_{\beta}(\lambda) e^{+i p \cdot x} b(p \lambda)^{\dagger}\right\}
\end{array}
$$

where $O^{s}(I) \equiv\left(\begin{array}{cc}D^{s}(L) & 0 \\ 0 & \bar{D}^{s}(I)\end{array}\right)$

$$
u(\lambda) \equiv\left(\begin{array}{l}
x_{\lambda} \\
x_{\lambda}
\end{array}\right) \quad, \quad v(\lambda) \equiv\left(\begin{array}{c}
x_{\lambda} \\
(-1)^{2 s} \\
x_{\lambda}
\end{array}\right)
$$

and $x_{\lambda} \equiv\left[\begin{array}{c}0 \\ \vdots \\ 0 \\ 1 \\ 0 \\ \vdots \\ 0\end{array}\right]$

were the 1 is in the $\lambda$ th now of $x_{\lambda}$. 
In terms of this field, the space-inversion, time-reversal, and charge-conjugation transformations are:

$$
\begin{aligned}
& \mathrm{u}\left(\rho_{s}\right) \psi(\mathrm{x}) \mathrm{U}\left(\ell_{\mathrm{s}}\right)^{\dagger}=\eta_{\mathrm{p}} \gamma_{0} \psi\left(\mathrm{I}_{\mathrm{s}} \mathrm{x}\right) \\
& \mathrm{u}\left(\ell_{\mathrm{t}}\right) \psi(\mathrm{x}) \mathrm{U}\left(\ell_{\mathrm{t}}\right)^{\dagger}=\mathcal{\theta}^{\mathrm{s}}\left(\zeta^{\dagger}\right) \psi\left(\mathrm{I}_{\mathrm{t}} \mathrm{x}\right)
\end{aligned}
$$

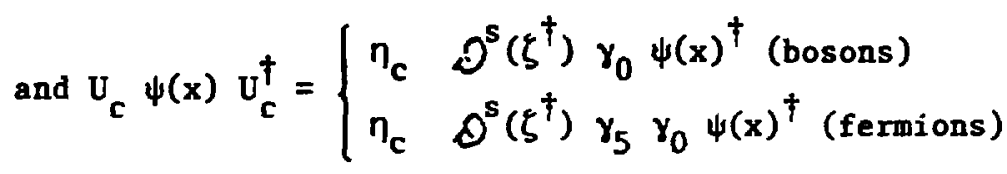

where $\gamma_{0} \equiv\left(\begin{array}{ll}0 & 1 \\ 1 & 0\end{array}\right)$ and $\gamma_{5}=\left(\begin{array}{cc}1 & 0 \\ 0 & -1\end{array}\right)$

\section{D-6 The Propogator}

The field-theoretic propogator is defined from Wick's theorem and the usual derivation of the Feynman rules as (for example, see Ref. 24)

$$
G_{\alpha \beta}(x-y) \equiv\left\langle 0\left|\left(\psi_{\alpha}(x) \psi_{\beta}(y)^{\dagger}\right)_{+}\right| 0\right\rangle
$$

with

$$
\left(\psi_{\alpha}(x), \psi_{\beta}(y)^{\dagger}\right)_{+}=\psi_{\alpha}(x) \psi_{\beta}(y)^{\dagger} \theta\left(x_{0}-y_{0}\right)+(-1)^{2 s} \psi_{\beta}(y)^{\dagger} \psi_{\alpha}(x) \theta\left(y_{0}-x_{0}\right)
$$


where $\theta\left(x_{0}-y_{0}\right)$ is a time-ordering step function and the factor $(-1)^{2 s}$ comes from the interchange of the fermion fields. The methods that were used in $\$ \mathrm{D}-2$ can then be applied to obtain

$$
\begin{aligned}
\left\langle 0\left|\left(\psi_{\alpha}(x) \psi_{\beta}(y)^{\dagger}\right)_{+}\right| 0\right\rangle & =\frac{1}{(2 \pi)^{3}} \int \frac{d^{3} p}{2 \varepsilon}\left\{N_{\alpha \beta}^{(+)}(p) e^{-i p \cdot(x-y)} \theta\left(x_{0}-y_{0}\right)\right. \\
+ & \left.(-1)^{2 s} N_{\alpha \beta}^{(-)}(p) e^{+i p \cdot(x-y)} \theta\left(y_{0}-x_{0}\right)\right\}
\end{aligned}
$$

where

$$
\begin{aligned}
\mathbb{N}_{\alpha \beta}^{(+)}(p) \equiv \sum_{\lambda \gamma \delta} \mathcal{O}_{\alpha \gamma}^{s}(C(p)) u_{\gamma}(\lambda) u_{\delta}(\lambda)^{*} D_{\delta \beta}^{s}\left(C(p)^{\dagger}\right) \\
=\left(\begin{array}{cc}
D^{s}\left(\frac{\sigma \cdot p}{m}\right) & 1 \\
1 & \bar{D}^{s}\left(\frac{\sigma \cdot p}{m}\right)
\end{array}\right)_{\alpha \beta}
\end{aligned}
$$

and

$$
\begin{aligned}
& N_{\alpha \beta}^{(-)}(p)=\sum_{\lambda \gamma \delta} D_{\alpha \gamma}^{s}\left(c(p) \zeta^{\dagger}\right) v_{\gamma}(\lambda) v_{\delta}(\lambda)^{\star} D_{\delta \beta}^{s}\left(\zeta c(p)^{\dagger}\right) \\
& =\left(\begin{array}{cc}
D^{s}\left(\frac{\sigma \cdot p)}{m}\right) & (-1)^{2 s} \\
(-1)^{2 s} & D^{s}\left(\frac{\sigma \cdot p}{m}\right)
\end{array}\right)_{\alpha \beta}
\end{aligned}
$$

Also note that Egs. $D-56$ and $D-57$ were obtained using $P_{0}=\varepsilon(|\vec{p}|)$. But, the expressions for the functions $N^{(+)}(p)$ and $y^{(-)}(p)$ are now polynomials in the four-momentum $p$, which implies that each component of the four-nomentum, $P_{\mu}$, in these polynomials can be changed to 
a derivative with respect to $(x-y)_{\mu}$ and the resulting differential expression can be renoved from the integral (cf., gD-2). Then, if the derivatives of the step functions are dropped, the propogator becomes

$$
G_{\alpha \beta}(x-y)=i \int \frac{d^{4} p}{(2 \pi)^{4}} N_{\alpha \beta}^{(+)}(p) \frac{1}{p^{2}-n^{2}+i \eta} e^{-i p \cdot(x-y)}
$$

where the Fourier transform of the step function (Eq. 4-28) has been used to convert this expression into an integral over the four-momentun $\mathbf{P}$.

Neglect of the step-function derivative terms in Eq. D-58 can be justified in two ways. First, it has been shown by Weinberg $^{28}$ that these terms are not covariant; thus, if a covariant theory is required, these terms must be dropped (i.e., the procedure can be stated as a basic postulate of the theory). In Lagrangian field theory, on the other hand, it can be shown that the stepfunction derivative terms are, in fact, cancelled by other noncovariant terms, which arise in the interaction Hamiltonian during the reduction from Lagrangian form (see Weinberg ${ }^{28}$ and references therein). (Note: An example of this cancellation - for the spin-1 field - is given in the text by Lurié. ${ }^{25}$ ) 


\section{D-7 The M Function}

The results of $\$ D-6$ can also be used to obtain a definition of the $M$ function (\$4.2) in terms of a field-theoretic vertex function. This is accomplished by rewriting Eqs. D-56 and D-57 as follows:

$$
N^{(+)}(p)=\sum_{\lambda}\left(\begin{array}{c}
x_{\lambda} \\
\bar{D}^{s}\left(\frac{\sigma \cdot p}{m}\right) x_{\lambda}
\end{array}\right) \otimes\left(x_{\lambda}^{\dagger} D^{s}\left(\frac{\sigma \cdot p}{m}\right), x_{\lambda}^{\dagger}\right)
$$

and

$$
N^{(-)}(p)=\sum_{\lambda}\left(\begin{array}{c}
x_{\lambda} \\
(-1)^{2 s} \bar{D}^{s}\left(\frac{\sigma \cdot p}{m}\right) x_{\lambda}
\end{array}\right) \otimes\left(x_{\lambda}^{\dagger} D^{s}\left(\frac{\sigma \cdot p}{m}\right),(-1)^{2 s} x_{\lambda}^{\dagger}\right)
$$

where $x_{\lambda}$ was defined in Eq. D-47 and the symbol $\otimes$ denotes the direct product.

i.e., $\left(\begin{array}{l}a \\ b\end{array}\right) \otimes\left(\begin{array}{ll}c & d\end{array}\right) \equiv\left(\begin{array}{ll}a c & a d \\ b c & b d\end{array}\right)$

Also note that the four-momentum $p$ in Eqs. D-56 and D-57 is the on-shell four-momentum (i.e., $P_{0}=\varepsilon(|\vec{p}|)$ ); bence, the complemenary set Iorentz transformation, $C(p)$, is well defined and 


$$
D^{s}\left(\frac{\sigma \cdot p}{m}\right) D^{s}\left(\frac{\sigma \cdot p}{m}\right)=D^{s}\left(\frac{\sigma \cdot p}{m}\right) D^{s}\left(\frac{\tilde{\sigma} \cdot p}{m}\right)=D^{s}\left(\frac{p^{2}}{m^{2}}\right)=1
$$

The procedure that was used in $\$ D-6$ can then be applied to obtain Eq. D-58; but, in this case, the function $N^{(+)}(p)$ is given by Eq. $D-60$ with $p_{0} \neq \varepsilon(|\vec{p}|)$.

i.e., $N^{(+)}(p)=\left(\begin{array}{cc}D^{s}\left(\frac{\sigma \cdot p}{m}\right) & 1 \\ D^{s}\left(\frac{p^{2}}{m^{2}}\right) & D^{s}\left(\frac{\sigma \cdot p}{m}\right)\end{array}\right)$

Eqs. D-56 and D-63 are not the same because equivalent on-shell expressions for the function $N^{(+)}(p)$ have been continued off-shell in different ways. Whether or not field theory is invariant under these different continuation procedures is currently being investigated.

The $M$ function is now defined using the function $N^{(+)}(p)$ that is given in Eq. D-60 (or, equivalently, D-63) to rewrite the field theoretic vertex function such that it includes the column vectors in that expression. For example, the field-theoretic vertex

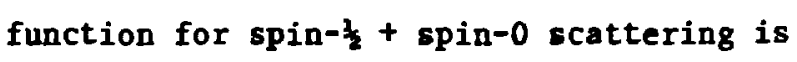

$$
\Gamma=\left(\begin{array}{ll}
\Gamma_{A A} & \Gamma_{A B} \\
\Gamma_{B A} & \Gamma_{B B}
\end{array}\right)
$$


where $\Gamma_{A A}, \Gamma_{B B}, \Gamma_{B A}$, and $\Gamma_{B B}$ are $2 \times 2$ matrices, and the $M$ function for this process is defined as

$$
\begin{aligned}
& M_{\lambda^{\prime} \lambda}=\left(x_{\lambda}^{\dagger}, D^{\frac{1}{2}}\left(\frac{\sigma \cdot p}{m}\right), x_{\lambda}^{\dagger}\right) \Gamma\left(\begin{array}{c}
x_{\lambda} \\
D^{\frac{1}{2}}\left(\frac{\sigma \cdot p}{m}\right) x_{\lambda}
\end{array}\right) \\
& =x_{\lambda^{\prime}}^{\dagger}\left\{D^{\frac{1}{2}}\left(\frac{\sigma \cdot p}{m}\right)\left(\Gamma_{A A}+\Gamma_{A B} D^{-\frac{1}{2}}\left(\frac{\sigma \cdot p}{m}\right)\right)+\left(\Gamma_{B A}+\Gamma_{\left.\left.B B^{-\frac{1}{2}}\left(\frac{\sigma \cdot p}{m}\right)\right)\right\} x_{\lambda}}\right.\right.
\end{aligned}
$$

This definition is equivalent to that shown in Eq. 4-10.

(Note: Weinberg ${ }^{28}$ has also shown how to construct fields that Lorentz transform via arbitrary representations of the Lorentz group $S L(2, C)$. That is, representations that are not $2(2 s+1)$ dimensional - e.g., the Rarita-Schwinger fields. An extension of the methods that have been developed in this appendix to a definition of the off-mass-shell $M$ function in terms of these fields and their corresponding vertex functions is expected to be a straightforward task and will not be discussed here.) 


\section{Appendix E}

Details from Chapter 6

\section{E-1 Proof of Eg. 6-69}

\section{Consider:}

$$
\begin{aligned}
& \int_{0}^{\infty} d\left(q_{1}^{\prime \prime}\right)_{0} \int_{0}^{\infty} d\left(p_{1}^{\prime \prime}\right)_{0} \int_{0}^{\infty} p_{1}^{\prime \prime 2} d p_{1}^{\prime} \int_{-1}^{1} d\left(\cos \theta_{1}^{\prime \prime}\right) \\
& \times \delta\left(\left(K_{1}^{\prime}\right)_{0}-\left(K_{1}^{\prime}\right)_{0}\right) \delta\left(\left(K_{2}^{\prime \prime}\right)_{0}-\left(K_{2}\right)_{0}\right) \frac{1}{q_{2}^{2}} \delta\left(q_{2}^{\prime \prime}-q_{2}\right)
\end{aligned}
$$

and make the variable change from $\left(\left(q_{1}^{\prime \prime}\right)_{0},\left(p_{1}^{\prime \prime}\right)_{0}, p_{1}^{\prime \prime}, \cos \theta_{1}^{\prime \prime}\right)$ to $\left(\left(K_{1}^{\prime}\right)_{0},\left(K_{2}^{\prime}\right)_{0}, q_{2}^{\prime \prime}, \cos \xi_{2}^{\prime}(1)\right)$ using the following equations:

$$
\begin{aligned}
& \left(K_{1}^{\prime \prime}\right)_{0}=-\left(q_{1}^{\prime \prime}\right)_{0}+\frac{1}{2}\left(1-\frac{1}{s}\left(m_{(1)}^{\prime}{ }^{2}-m_{1}^{2}\right)\right) \underset{\sim}{W}: s \equiv W^{2} \\
& m_{(1)}^{\prime}=\left(\left|\Delta_{(1)}^{\prime}{ }^{2}\right|+m_{2}^{2}\right)^{\frac{1}{2}}+\left(\left|\Delta_{(1)}^{\prime}{ }^{2}\right|+m_{3}^{2}\right)^{\frac{1}{2}} \\
& \Delta_{(1)}^{\prime \prime}{ }^{2}=\left(p_{1}^{\prime \prime}\right)_{0}^{2}-p_{1}^{\prime}{ }^{2} \\
& \left(K_{2}^{\prime \prime}\right)_{0}=\left(k_{2(1)}^{\prime}\right)_{0} \cosh x_{(1)}^{\prime}+p_{1}^{\prime} \sinh x_{(1)}^{\prime} \cos \theta_{1}^{\prime} \\
& q_{2}^{\prime \prime} \sin \xi_{2}^{\prime \prime}(1)=p_{1}^{\prime \prime} \sin \theta_{1}^{\prime \prime}
\end{aligned}
$$




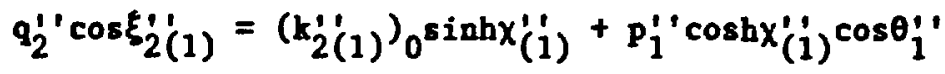

$$
\begin{aligned}
& \cosh x_{(i)}^{\prime}=\frac{\left(K_{(i)}^{\prime}\right)_{0}}{{\underset{\sim}{w}}_{(1)}^{\prime}} \quad \sinh x_{(1)}^{\prime}=\frac{q_{1}^{\prime}}{w_{(i)}^{\prime}} \\
& \underline{w}_{(i)}^{\prime}=\left((K(i))_{0}^{2}-\dot{q}_{1}^{\prime \prime 2}\right)^{\frac{1}{2}} \\
& \left(k_{2(1)}^{\prime \prime}\right)_{0}=\left(p_{1}^{\prime \prime}\right)_{0}+\frac{1}{2}\left(1+\frac{1}{s_{1}^{\prime \prime}}\left(m_{2}{ }^{2}-m_{3}{ }^{2}\right)\right){\underset{\sim}{w}}_{(1)}^{\prime}: s_{1}^{\prime \prime} \equiv{\underset{\sim}{w}}_{(1)}^{\prime}{ }^{2} \\
& W=\left(K_{(i)}^{\prime}\right)_{0}+\left(K_{i}^{\prime \prime}\right)_{0}
\end{aligned}
$$

where $q_{1}^{\prime \prime}=q_{1}^{\prime}$ and $\underset{\sim}{W}$ are constants. These equations can be used to show that

$$
\begin{aligned}
& \frac{\partial\left(\left(K_{1}^{\prime}\right)_{0},\left(K_{2}^{\prime}\right)_{0}, q_{2}^{\prime}, \cos \xi_{2(1)}^{\prime}\right)}{\left.\partial\left(\left(q_{1}^{\prime}\right)_{0},\left(p_{1}^{\prime}\right)_{0}, P_{1}^{\prime}, \cos \theta_{1}^{\prime}\right)^{\prime}\right)}
\end{aligned}
$$

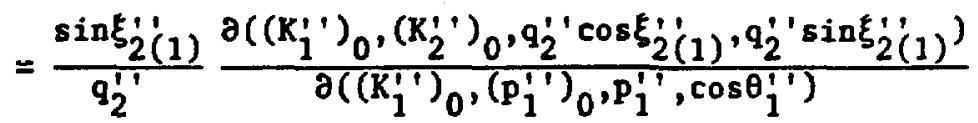

$$
\begin{aligned}
& =-\frac{\sin \xi_{2(1)}^{\prime \prime}}{q_{2}^{\prime \prime}} \frac{p_{1}^{\prime \prime}}{\sin \theta_{1}^{\prime \prime}} \\
& =\left(\frac{p_{1}^{\prime}}{q_{2}^{\prime \prime}}\right)^{2}
\end{aligned}
$$

Thus, the integral in Eq. E-1 is equal to

$$
\int_{-1}^{1} d\left(\cos _{2}^{\prime \prime}(1)\right)
$$




\section{E-2 Proof of Eg. 6-22}

Consider:

$\left\langle P^{\prime}\left[q_{1}^{\prime} J^{\prime} M^{\prime}\left(p_{1}^{\prime} j_{1} m_{1} \lambda_{2}^{\prime} \lambda_{3}^{\prime}\right) \lambda_{1}^{\prime}\right] l P\left[q_{2} J M\left(p_{2} j_{2} m_{2} \lambda_{3} \lambda_{1}\right) \lambda_{2}\right]\right\rangle$

$$
\begin{aligned}
& =\frac{1}{4 \pi}\left(\frac{2 j_{1}+1}{4 \pi}\right)^{\frac{3}{2}}\left(\frac{2 j_{2}+1}{4 \pi}\right)^{\frac{3}{2}} e^{i \pi s_{3}-i \pi s_{2}} \delta_{J^{\prime} J} \delta_{M^{\prime} M} \\
& \times \int d^{2} \hat{q}_{1}^{\prime} d^{2} \hat{q}_{2} d^{2} \hat{p}_{1}^{\prime} d^{2} \hat{p}_{2} D_{m_{2}-\lambda_{2}}^{J}, m_{1}-\lambda_{1}^{\prime}\left(R\left(\hat{q}_{2}\right)^{-1} R\left(\hat{q}_{1}\right)\right) \\
& \times D_{m_{1}, \lambda_{2}^{\prime}-\lambda_{3}^{\prime}}^{j_{1}}\left(R\left(\hat{p}_{1}^{\prime}\right)\right) D_{m_{2}, \lambda_{3}-\lambda_{1}}^{j_{2}}\left(R\left(\hat{p}_{2}\right)\right)^{*} \\
& \times D_{\lambda_{1}^{\prime} \lambda_{1}}^{s_{1}}\left(C\left(K_{1}\right)^{-1} C\left(R_{(2)}\right) C\left(k_{1(2)}\right)\right) D_{\lambda_{2}^{*} \lambda_{2}}^{s_{2}}\left(C\left(k_{2}(1)\right)^{-1} C\left(K_{(1)}\right)^{-1} C\left(K_{2}\right)\right) \\
& \times \mathrm{D}_{\lambda_{3}^{+} \lambda_{3}}^{s_{3}}\left(C\left(k_{3(1)}\right)^{-1} \mathrm{C}\left(\mathrm{K}_{(1)}\right)^{-1} \mathrm{C}\left(\mathrm{K}_{(2)}\right) \mathrm{C}\left(\mathrm{k}_{3(2)}\right)\right)
\end{aligned}
$$

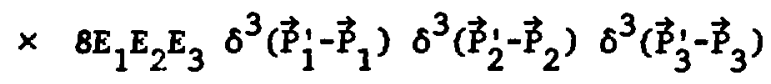

with $\delta^{3}\left(\vec{P}_{1}^{\prime}-\vec{P}_{1}\right) \delta^{3}\left(\vec{P}_{2}^{\prime}-\vec{P}_{2}\right) \delta^{3}\left(\vec{P}_{3}^{\prime}-\vec{P}_{3}\right)=\frac{E}{E_{1} E_{2} E_{3}} \frac{w_{3(2)^{W}} w_{1(2)} W_{(2)} W_{2}}{{ }^{W}(2)^{W}}$

$$
\times \delta^{3}(\overrightarrow{\mathrm{p}} \cdot-\overrightarrow{\mathrm{p}}) \delta^{3}\left(\overrightarrow{\mathrm{q}}_{2}^{\prime}-\overrightarrow{\mathrm{q}}_{2}\right) \delta^{3}\left(\overrightarrow{\mathrm{p}}_{2}^{\prime}-\overrightarrow{\mathrm{p}}_{2}\right)
$$


and $\delta^{3}\left(\vec{q}_{2}^{\prime}-\vec{q}_{2}\right) \delta^{3}\left(\vec{p}_{2}^{\prime}-\vec{p}_{2}\right)=\frac{1}{q_{2}{ }^{2} p_{2}{ }^{2}} \frac{{ }^{w}(2) p_{2}}{w_{3(2)}{ }_{1(2)}} \frac{q_{2}}{w_{(2)} w_{2}}$

$$
\times \delta\left(W^{\prime}-W\right) \delta\left(w_{(2)}^{\prime}{ }^{-w}(2) \delta^{2}\left(\hat{q}_{2}^{\prime}-\hat{q}_{2}\right) \delta^{2}\left(\hat{p}_{2}^{\prime}-\hat{p}_{2}\right) \quad(E-16)\right.
$$

and $\delta^{3}\left(\vec{P}^{\prime}-\vec{P}\right) \delta\left(W^{\prime}-W\right)=\frac{W}{E} \delta^{4}\left(P^{\prime}-P\right)$

which imply that

$$
\begin{aligned}
& 8 E_{1} E_{2} E_{3} \delta^{3}\left(\vec{P}_{1}^{\prime}-\vec{p}_{1}\right) \delta^{3}\left(\vec{P}_{2}^{\prime}-\vec{p}_{2}\right) \delta^{3}\left(\vec{P}_{3}^{\prime}-\vec{p}_{3}\right) \\
& =8 W \frac{1}{q_{2} p_{2}} \delta^{4}\left(P^{\prime}-P\right) \delta\left(w_{(2)}^{\prime}{ }^{-w}(2)\right) \delta^{2}\left(\hat{q}_{2}^{\prime}-\hat{q}_{2}\right) \delta^{2}\left(\hat{p}_{2}^{\prime}-\hat{p}_{2}\right)
\end{aligned}
$$

Hence, the integrals over $\hat{q}_{2}^{\prime}$ and $\hat{p}_{2}^{\prime}$ in Eq. E-14 can be completed. Eqs. 6-36 through 6-38 and Eqs. 6-43 through 6-45 can then be used to show that

$$
w_{(2)} w_{1}=w_{1(2)} w_{(2)}-p_{2} q_{2} \cos \theta_{2}
$$

and $w_{(1)} w_{2}=w_{2(1)} W_{(1)}+p_{1} q_{1} \cos \theta_{1}$

with (cf., Eq. 3-7) $q_{2}=\frac{1}{2 W} \xi\left(w^{2}, w(2){ }^{2}, m_{2}{ }^{2}\right.$ )

These equations imply that

$$
\frac{\partial \cos \theta_{1}}{\partial w_{(2)}}=\frac{w_{(1)}}{P_{1} q_{1}} \frac{\partial w_{2}}{w_{(2)}}=\frac{w_{(1)}}{P_{1} q_{1}} \frac{q_{2}}{W_{2}} \frac{\partial q_{2}}{\partial w_{(2)}}=\frac{w_{(1)}{ }^{w}(2)}{P_{1} q_{1} w}
$$


Hence, $\quad \delta\left(w_{(2)}^{\prime}{ }^{-w}(2)=\frac{w_{(1)} w_{(2)}}{p_{1} q_{1} W} \delta\left(\cos \theta_{1}-\frac{w_{(1)} W_{2}-w_{2(1)} W(1)}{p_{1} q_{1}}\right)\right.$ (E-23)

Furthermore, the analysis that was presented in \$6.3.1 showed that the integrand in Eq. E-5 is independent of the angles $\Phi_{1}, \underline{\theta}_{1}$, and $\phi_{1}$. Thus, the integrals over these angles can be completed to obtain the on-shell recoupling coefficient. The result is equal to

$$
8 \frac{w^{w}(1)^{w}(2)}{q_{1} p_{1} q_{2} p_{2}} \delta^{4}\left(P^{\prime}-P\right) \delta_{J} J^{\prime} \delta_{M^{\prime} M}
$$

times the on-shell $T$ function recoupling coefficient, which is given in Eq. 6-79. 


\section{ACKNOWLEDGMENTS}

I would like to thank ny research supervisor Willian Gibbs, the staff members, and the post-docs of Group T-5 at the Los Alamos Scientific Laboratory for their support and encouragement during my dissertation research. In addition, I would like to acknowledge the many people at LASL who bave contributed to my education. I am especially indebted to Mikkel Johnson and James Louck for many enlightening conversations. I am also grateful for the friendship of Frank Cverna and Doug Weiss. 


\section{REFERENCES}

1. for example, see Meson-Nuclear Physics - 1979, E. V. Hungerford, ed., AIP Conf. Proc. 54 (1979).

2. E. J. Moniz, "Pion-Nucleus Interactions," Lectures delivered at the Les Houches Sumer School on Nuclear Physics with Heavy Ions and Hesons, July, 1977, Les Houches, France.

J. Hufner, "Pions Interact witb Nuclei", Phys. Rep. 21C, 1 (1975).

3. I. Celenza, I. C. Liu, and C. M. Shakin, "Covariant PionNucleus Optical Potential", Phys. Rev. C 11, 1593 (1975), and references therein.

4. J. M. Namyslowski, "Decoupled and Covariantly Reduced Relativistic Fadeev Equations," Nuov. Cim. 57, 355 (1968).

5. D. Robson, "Many-Body Interactions from Quark Exchanges and the Tetrahedral Crystal Structure of Nuclei," Nucl. Phys. A $\underline{308}$, 381 (1978).

6. J. R. Taylor, Scattering Theory, John Wiley \& Sons., Inc., $\mathrm{NI}$ (1972).

C. J. Joachain, Quantum Collision Theory, North-Holland Publishing Co., Amsterdam, Holland (1975).

C. J. Joachain and C. Quigg, "Hultiple-Scattering Expansions in Several-Particle Dyaamics", Rev. of Mod. Phys. 46, 279 (1974).

M. L. Goldberger and K. M. Watson, Collision Theory, John Wiley \& Sons., Inc., New York, NY (1964).

7. T. F. Jordan, Linear Operators for Quantum Hechanics, John Wiley \& Sons., Inc., New York, WY (1969).

8. B. Bakamjian and L. H. Thomas, "Relativistic Particle Dynanics. II," Phys. Rev. 92, 1300 (1953).

I. I. Foldy, "Relativistic Particle Systens with Spin", Phys. Rev. 122, 275 (1961). 
T. F. Jordan, A. J. Macfarlane, and E. C. C. Sudarshan, "Hamiltonian Model of Lorentz Invariant Particle Interactions", Phys. Rev. B 133, 487 (1964).

H. Osborn, "Relativistic Center-of-Mass Variables for TwoParticle Systems with Spin", Phys. Rev. 176, 1514 (1968).

R. A. Krajcik and L. I. Foldy, "Relativistic Center-of-Mass Variables for Composite Systems with Arbitrary Internal Interactions", Phys. Rev. D 10, 1777 (1974).

L. Heller, "The Relativistic Schrodinger Equation", IASL report, IA-5594-MS (1974).

F. Coester and P.Havas, "Approximately Relativistic Hamiltonians for Interacting Particles", Phys. Rev. D 14, 2556 (1976).

F. Coester, "Canonical Scattering Theory for Relativistic Particles", preprint.

F. Rohrlich, "Relativistic Hamiltonian Dynamics", preprint.

9. M. V. Barnhill, "Ambiguity of the Galilean-Invariant Operator for Pion-Absorption by Nuclei", Nucl. Phys. A 131, 106 (1969).

M. Bolsterli, et al., "Galilean-Invariance Ambiguity in the Nonrelativistic Pion-Nucleon Absorption Operator", Phys. Rev. C 10,1225 (1974).

J. I. Friar, "Pion-Nucleon Absorption Operator Ambiguity", Phys. Rev. C 10, 955 (1974).

10. D. J. Ernst and G. A. Miller, "Relativistic Kinematics for Elastic Pion-Nucleus Scattering", preprint, and references therein.

- also see -

L. Heller, "Relativistic Description of Directly Interacting Pions and Nucleons", LASL report, LA-UR-76-1197 (1976).

I. Heller, G. E. Bohannon, and F. Tabakin, "Dependence of the Off-Energy-Shell $T$ Matrix on the Total Three-Monentum", Phys. Rev. C 13, 742 (1976).

H. Garcilazo, "Frame Dependence of the Off-Energy-Shell T Matrix", Phys. Rev. C 16, 1996 (1977). 
11. V. B. Mandelzweig, H. Garcilazo, and J. M. Eisenberg, "PionDeuteron Scattering in a Relativistic Three-Body Theory", Nucl. Phys. A 256, 461 (1976).

12. R. Aaron, R. D. Amado, and J. E. Young, "Relativistic ThreeBody Theory with Applications to $\pi N$ Scattering", Phys. Rev. 174,2022 (1968).

R. M. Woloshyn, E. J. Moniz, and R. Aaron, "Relativistic ThreeBody Calculation of $\pi d$ Scattering", Phys. Rev. C 13, 286 (1976).

R. Aaron, "A Relativistic Three-Body Theory", in Modern ThreeHadron Physics, ed. A. W. Thomas, Springer-Verlag, New York, NY (1977).

R. R. Silbar and W. M. Kloet, "Nucleon-Nucleon Dynamics at Medium Energies. I: Unitary Model for Elastic and Inelastic Scattering", LASI report, LA-UR-79-2138 (1979).

13. G. F. Chew, S-Matrix Theory of Strong Interactions, $W$. A. Benjamin, Inc., New York, NY (1961).

D. Amati and S. Fubini, "Dispersion Relation Methods in Strong Interactions", Ann. Rev. of Nucl. Sc. 12, 359 (1962).

G. F. Chew, The Analytic S Matrix, W. A. Benjamin, Inc., New York, NY (1966).

R. J. Eden, et al., The Analytic S Matrix, Canbridge Univ. Press, London, England (1966).

R. J. Eden, High-Energy Collisions of Elementary Particles, Cambridge Univ. Press, London, England (1967).

H. Burkhadt, Dispersion Relation Dynamics, North-Holland Publishing Co., Amsterdam, Holland (1969).

14. H. P. Stapp, "Lectures on the S-Matrix Theory", LRL Report, UCRL-9875 (1961).

A. O. Barut, "Formulation of the Scattering Functions in Terms of the Unitary Representations of the Inhomogeneous Lorentz Group", Phys. Rev. 127, 321 (1962).

I. J. Muzinich, "Crossing Matrices for Helicity Aplitudes, Application to Crossed-Channel Partial-Wave Analysis, and Resgeization", J. of Math. Phys. 5, 1481 (1964). 
H. P. Stapp, "Analyticity Properties of Helicity Amplitudes", Phys. Rev. 160, 1251 (1967).

A. 0. Barut, The Theory of the Scattering Matrix, McMillian Co., New York, N $\bar{Y}(1967)$.

- also see -

J. R. Taylor, "Spin and Isospin in S-Matrix Theory", J. of Math. Phys. I, 181 (1966).

15. A. D. Martin and T. D. Spearman, Elementary Particle Theory, North-Holland Publishing Co., Amsterdam, Holland (1970).

16. H. P. Stapp, "Derivation of the CPT Theorem and the Connection between Spin and Statistics from Postulates of the S-Matrix Theory", Phys. Rev. 125, 2139 (1962).

17. H. P. Stapp, "Space and Time in S-Matrix Theory", Phys. Rev. B 139,257 (1965).

18. A. O. Barut, I. J. Muzinich, and D. N. Williams, "Construction of Invariant Scattering Amplitudes for Arbitrary Spins and Analytic Continuation in Total Angular Momentum", Phys. Rev. 130,442 (1963).

19. A. C. Hearn, "The Invariant Amplitudes of Interaction Processes", Nuov. Cim. 21, 333 (1961).

20. J. D. Jackson and G. E. Hite, "Kinematic Singularities and Threshold Relations for Helicity Amplitudes", Phys. Rev. 169, 1248 (1968).

21. Y. Hara, "Analyticity Properties of Helicity Amplitudes and Construction of Kinematical Singularity-Free Amplitudes for Any Spin", Phys. Rev. B 136, 507 (1964).

G. Cohen-Tannoudji, A. Morel, and H. Navelet, "Kinematic Singularities, Crossing Matrix and Kinematical Constraints for Two-Body Helicity Amplitudes", Ann. of Phys. 46, 239 (1968).

I-I. C. Wang, "General Method of Constructing Helicity Amplitudes Free from Kinematic Singularities and Zeros", Phys. Rev. 142, 1187 (1966).

G. C. Fox, "Methods for Constructing Invariant Anplitudes Free from Kinematic Singularities and Zeros", Phys. Rev. 157, 1493 (1967). 
- also see -

T. L. Truenan and G. C. Wick, "Crossing Relations for Helicity Anplitudes", Ano. of Phys. 26, 322 (1964).

22. J. J. Brehn, "Unitarity and the Isobar Model", Ann. of Phys. 108,454 (1977).

23. S. Gasiorowitz, Elenentary Particle Physics, John Wiley \& Sons., Inc., New York, W (1966).

24. P. Ronan, Introduction to Quantur Field Theory, John Wiley \& Sons., Inc., New York, NY (1969).

25. D. Lurié, Particles and Fields, John Wiley \& Sons., Inc., New York, NY (1968).

26. R. F. Streater and A. S. Wightman, PCT, Spin and Statistics, and All That, W. A. Benjamin, Inc., New York, NY (1964).

27. W. knrit and J. Schwinger, "On a Theory of Particles with Half-Integer Spin", Phys. Rev. 60, 61 (1941).

28. S. Weinberg, "Teynman Rules for Any Spin", Phys. Rev. B 133, 1318 (1964).

S. Weinberg, "Feynman Rules for Any Spin. II. Massless Particles", Phys. Rev. B 134, 882 (1964).

29. P. A. Carruthers, Spin and Isospin in Particle Physics, Gordon and Breach, Science Publishers, Inc. (1971), and references therein.

30. V. Bargmann and E. P. Wigner, "Group Theoretical Discussion of Relativistic Wave Equations", Proc. of the Nat'1. Acad. of Sci. 34, 211 (1946).

W. L. Bade and H. Jehle, "An Introduction to Spinors", Rev. of Hod. Phys. 25, 714 (1953).

31. G. Feinberg and S. Weinberg, "On the Phase Factors in Inversions", Nuov. Cim. 14, 571 (1959).

32. A. Klein and C. Zenach, "Many-Body Probler: in Quantu Field Theory", Phys. Rev. 108, 126 (1957). 
33. N. Nakaniski, "A General Survey of the Bethe-Salpeter Equation", Supp. Prog. in Theor. Phys. (1969).

- also see -

K. Ladanyi, "Expansion Methods for the Spinor-Spinor BetheSalpeter Equation", Ann. of Phys. 77, 471 (1973).

G. C. Wick, "Properties of Bethe-Salpeter Wave Functions", Phys. Rev. 96, 1124 (1954).

R. E. Cutkosky, "Solutions of the Bethe-Salpeter Equation", Phys. Rev. 96, 1135 (1954).

34. E. Wigner, "On Unitary Representations of the Inhomogeneous Lorentz Group", Ann. of Math. 40, 39 (1939).

35. A. J. Macfarlane, "On the Restricted Lorentz Group and Groups Homomorphically Related to It", J. of Math. Phys. $\underline{3}, 1116$ (1962).

36. A. S. Wightman, "Lectures on Invariance in Relativistic Quantum Mechanics", Les Houches Lecture Series, Les Houches, France (1960).

37. A. J. Macfarlal.2, "Kinematics of the Relativistic Two-Particle System", J. of Math. Phys. 4, 490 (1963).

38. M. Carmeli and $S$. Malin, Representations of the Rotation and Lorentz Groups, Marcel Dekker, Inc., New York, NY (1976).

39. V. Bargmann, "Note on Wigner's Theorem on Sywmetry Operations", J. of Math. Phys. 5, 862 (1964).

40. R. M. F. Houtappel, "The Conceptual Basis and Use of the Geonetric Invariance Principles", Rev. of Mod. Phys. 37, 595 (1965).

41. I. C. Biedenharn and J. D. Louck, Angular Momentum in Quantum Physics. Encyclopedia of Mathematics and Its Applications, Vol. 8, Addison-Wesley, Reading, MA (1980).

42. A. R. Ednonds, Angular Momentum in Quantum Mechanics, Princeton Univ. Press, Princeton, HJ (1957).

43. H. E. Rose, Elenentary Theory of Angular Monentum, Wiley, New York, WY (1957). 
44. A. Messiah, Quantum Hechanics: Volume II, Appendix C, NorthHolland Publishing Co., Ansterdan, Holland (1966).

45. F. R. Halpern, Special Relativity and Quantum Mechanics, Prentice-Hall, Inc., Englewood, NJ (1968).

46. W. M. Gibson and B. R. Pollard, Symetry Principles in Elenentary Particle Physics, Canbridge Univ. Press, London, England (1976).

47. M. Jacob and G. C. Wick, "On the General Theory of Collisions for Particles with Spin", Ann. of Phys. I, 404 (1959).

48. G. C. Wick, "Angular-Monentum States for Three Relativistic Particles", Ann. of Phys. 18, 65 (1962).

49. J. M. Kamyslowski, M. S. K. Razmi, and R. G. Roberts, "Analysis of 3-Body Angular Monentum States in $\pi N \rightarrow \pi \pi N "$, Phys. Rev. 157, 1328 (1967).

50. A. J. Macfarlane, "Relativistic Partial Wave Analysis", Rev. of Mod. Phys. 34, 41 (1962).

A. J. Macfarlane, "Invariant Angular Functions for a Production Reaction Involving Spinless Particles", Nucl. Phys. 38, 504 (1962).

51. R. Balian and E. Brezin, "Angular-Monentum Reduction of the Fadeev Equations", Nuov. Cim. 61, 403 (1969).

52. M. Baranger, "Recent Progress in the Understanding of Finite Nuclei from the Two-Nucleon Interaction", Proc. Int. School of Phys. Enrico Fermi, Course 40, Varenna, Italy (1967)

M. B. Johnson and M. Baranger, "Folded Diagrams", Ann. of Phys. 62,172 (1971).

M. B. Johnson, "Theory of Meson-Exchange Potentials for Xuclear Physics", Ann. of Phys. 97, 400 (1976).

53. R. Blankenbecler and R. Sugar, "Linear Integral Equations for Relativistic Multichannel Scattering", Phys. Rev. 142, 1051 (1966). 
L. D. Landau, "On Analytic Properties of Vertex Parts in Quantun Field Theory", Nucl. Phys. 13, 181 (1959).

R. E. Cutkosky, "Singularities and Discontinuities of Feynman Amplitudes", J. of Math. Phys. 1, 429 (1960).

R. E. Cutkosky, "Some Applications of the Generalized Unitarity Relation", Phys. Rev. Lett. 4, 624 (1960).

R. E. Cutkosky, "Wave Functions", Phys. Rev. 125, 745 (1962). and Ref. 11 (R. J. Eden, et al.)

54. J. H. Namyslowski, "3-Dimensional, 2-Body Integral Equations. On-Shell and Off-Shell Formalisms", Phys. Rev. 160, 1522 (1967).

55. D. J. Ernst and M. B. Johnson, "Pion-Nucleon Form Factor in the Chew-Low Theory", Phys. Rev. C 17, 247 (1978).

56. M. J. Reiner, "A Theory of the Off-Energy-Shell Pion-Nucleon T Matrix", preprint.

57. I. J. R. Aitchison and J. J. Brehn, "Unitary Analytic Isobar Model for the Reaction Nucleon-Meson to Nucleon-Nucleon-Meson", Phys. Rev. D 17, 3072 (1978).

58. J. D. Jackson, Classical Electrodynamics, John Wiley \& Sons, Inc., New York, NY (1975).

59. A. 0. Barut and R. Raczka, Theory of Group Representations and Applications, Polish Scientific Publishers, Warszawa, Poland (1977).

60. R. Aaron and R. D. Amado, "Relativistic Isobar Model: Spinless Particles", Phys. Rev. D 13, 2581 (1976).

61. J. M. Namyslowski, "Clustering, The Necessary and Sufficient Conditions", contributed paper, Proceedings of the Ninth International Conference on the Few-Body Problem, Eugene, OR (1980). 